\title{
Evaluation of Concepts for Multiple Application Thermal Reactor for Irradiation eXperiments (MATRIX)
}

Michael A. Pope Hans D. Gougar John M. Ryskamp

September 2013
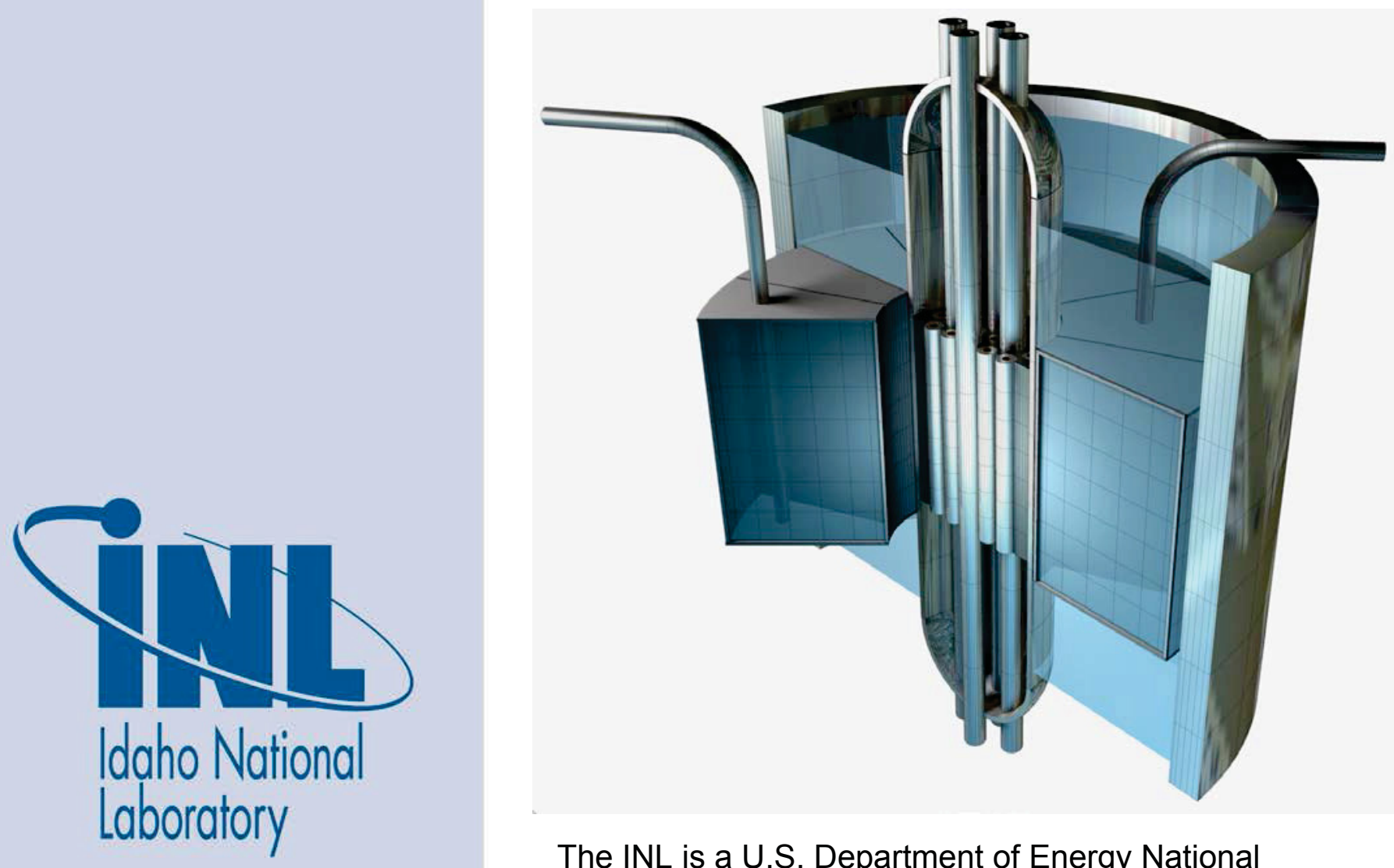

The INL is a U.S. Department of Energy National Laboratory operated by Battelle Energy Alliance 


\section{DISCLAIMER}

This information was prepared as an account of work sponsored by an agency of the U.S. Government. Neither the U.S. Government nor any agency thereof, nor any of their employees, makes any warranty, expressed or implied, or assumes any legal liability or responsibility for the accuracy, completeness, or usefulness, of any information, apparatus, product, or process disclosed, or represents that its use would not infringe privately owned rights. References herein to any specific commercial product, process, or service by trade name, trade mark, manufacturer, or otherwise, does not necessarily constitute or imply its endorsement, recommendation, or favoring by the U.S. Government or any agency thereof. The views and opinions of authors expressed herein do not necessarily state or reflect those of the U.S. Government or any agency thereof. 


\title{
Evaluation of Concepts for Multiple Application Thermal Reactor for Irradiation eXperiments (MATRIX)
}

\author{
Michael A. Pope \\ Hans D. Gougar \\ John M. Ryskamp
}

September 2013

Idaho National Laboratory Idaho Falls, Idaho 83415

http://www.inl.gov

Prepared for the

U.S. Department of Energy

Office of Nuclear Energy

Under DOE Idaho Operations Office

Contract DE-AC07-05ID14517 



\title{
Evaluation of Concepts for Multiple Application Thermal Reactor for Irradiation eXperiments (MATRIX)
}

\author{
INL/EXT-13-30045
}

September 2013

Approved by:

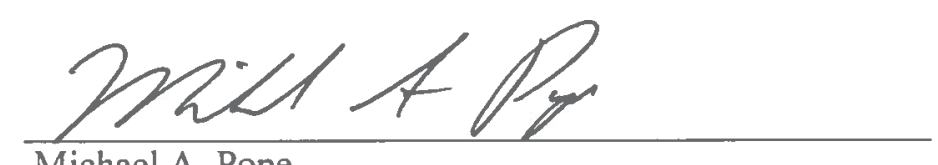

Michael A. Pope

Reactor Physics Analysis and Design

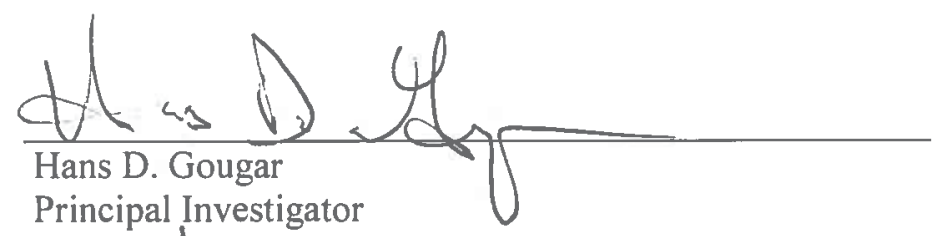

Principal Investigator

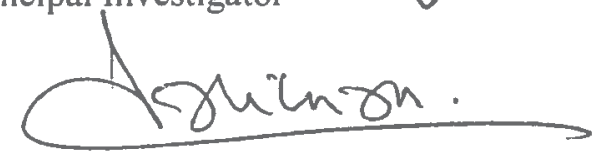

Gilles Youinou

Manager, Reactor Physics Analysis and Design
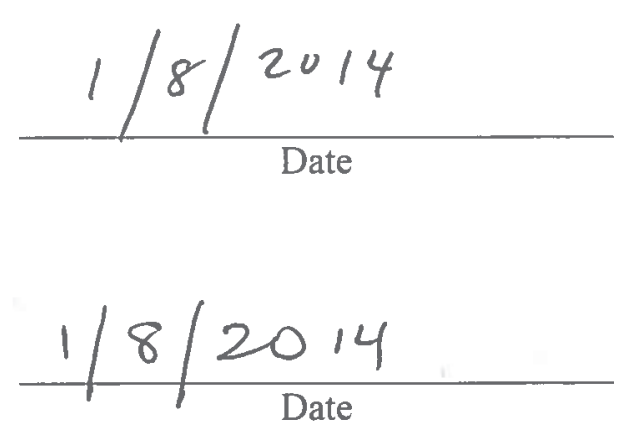

$\frac{1 / 8 / 2014}{\text { Date }}$ 


\section{ABSTRACT}

The Advanced Test Reactor (ATR) is a high power density test reactor designed primarily for accelerated irradiation of nuclear fuels and materials irradiation under controlled environmental conditions. For more than 45 years, the ATR has operated primarily in support of the Office of Naval Reactors (NR) but the mission has gradually expanded to cater to other customers, such as the DOE Office of Nuclear Energy (NE), private industry, and universities. . Featuring nine large flux traps that can accommodate independently-cooled high-pressure In-Pile Tubes (IPTs), ATR is still reliable and effective but increasingly expensive to maintain and operate every year. The sophisticated test hardware and experiments required of recent fuel and materials irradiation campaigns are limited by the safety limits and operating envelope of this aging facility, which are becoming harder to meet as equipment reliability is diminished. Furthermore, the fabrication and transport of its highly enriched uranium (HEU) fuel is deemed a proliferation concern. Under the Global Threat Reduction Initiative (GTRI), ATR and the other high flux test reactors in the US fleet must eventually be converted to low enriched uranium (LEU) in order to continue operating. Studies are underway to assess the costs and technical viability of this fuel conversion. One foreseeable outcome is that the cost of upgrades, fuel conversion, and more frequent LEU fuel loadings needed to keep ATR viable will exceed those of building and operating a replacement reactor. In anticipation of this scenario, work was commenced under the Laboratory Directed Research and Development (LDRD) program to investigate test reactor concepts that could satisfy the current missions of the ATR. Naturally, the needs of other value-adding missions were considered as part of the initial design study.

This work build upon a project from the 1990's called the Broad Application Test Reactor (BATR). In FY 2012, a survey of anticipated customer needs was performed, followed by analysis of the original BATR concepts with fuel changed to low-enriched uranium. Departing from these original BATR designs, four concepts were identified for further analysis in FY2013. The project informally adopted the acronym MATRIX (Multiple-Application Thermal Reactor for Irradiation eXperiments). This report discusses analysis of the four MATRIX concepts along with a number of variations on these main concepts. Designs were evaluated based on their satisfaction of anticipated customer requirements and the "Cylindrical" variant was selected for further analysis of options. This downselection should be considered preliminary and the backup alternatives should include the other three main designs.

The analysis described within indicates that the baseline Cylindrical MATRIX design can achieve a higher burnup than the ATR (or longer cycle length given a particular batch scheme). The volume of test space in IPTs is larger in MATRIX than in ATR with comparable magnitude of neutron flux. In addition to the IPTs, the this concept features test spaces at the centers of fuel assemblies where very high fast flux can be achieved. This magnitude of fast flux is similar to that achieved in the ATR A-positions, however, the available volume having these conditions is greater in the MATRIX design than in the ATR.

The Cylindrical MATRIX design can meet the anticipated needs of current and anticipated ATR customers This statement must be qualified by acknowledging that this design is quite

immature, however, and any requirements currently met must be re-evaluated as the design matures. Also, some of the requirements were not strictly met, but are believed to be achievable once features to be added later are designed. 


\section{CONTENTS}

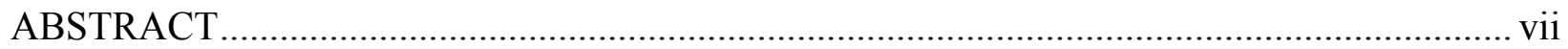

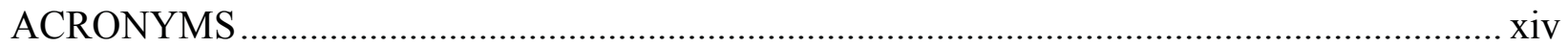

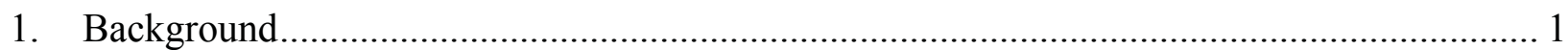

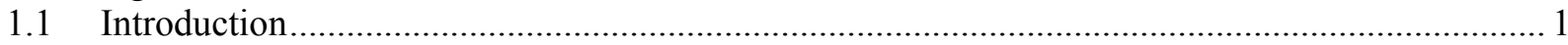

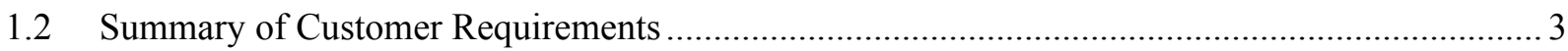

1.3 Reactor Physics Calculation Methodology ………………………………………………... 4

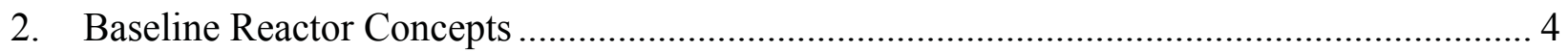

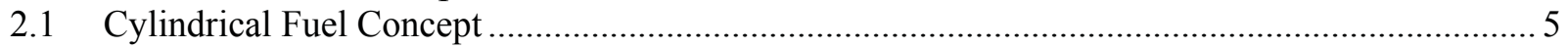

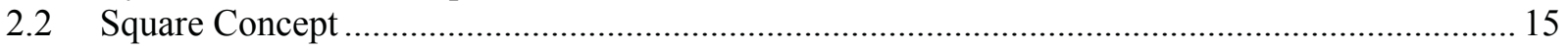

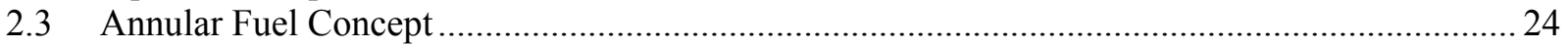

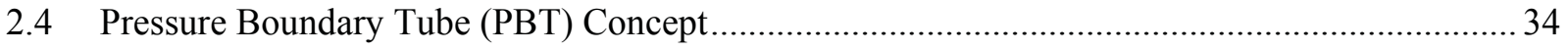

3. Pressure Vessel and PBT Considerations .......................................................................... 41

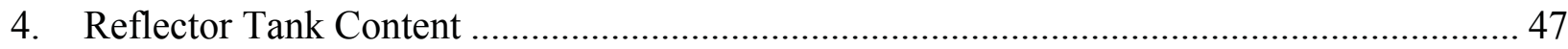

4.1 General Considerations for Reflector Tank Content................................................................ 47

$4.2 \quad$ Replaceable, Reconfigurable $\mathrm{D}_{2} \mathrm{O}$ Tanks....................................................................... 47

4.3 Performance Comparison.................................................................................................... 52

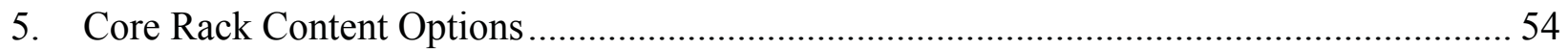

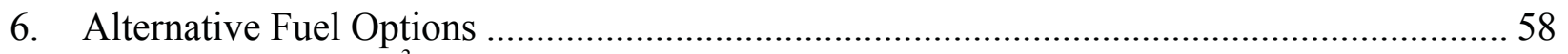

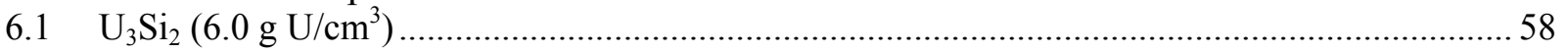

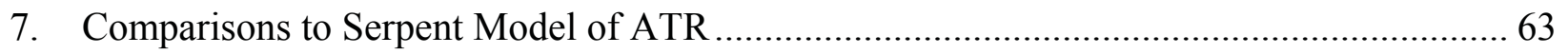

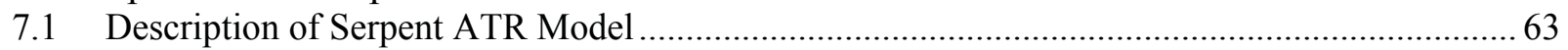

7.2 Initial Results of ATR Depletion Analysis ..............................................................................6 66

7.3 Comparison of Fast-to-Thermal Flux Ratios ………………………………………………....6 67

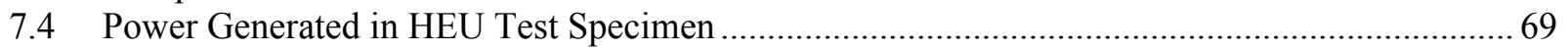

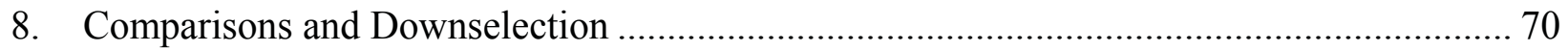

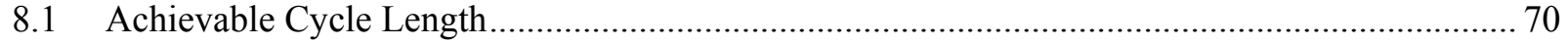

8.2 High Availability/Capacity Factor ………………………………………………………. 73

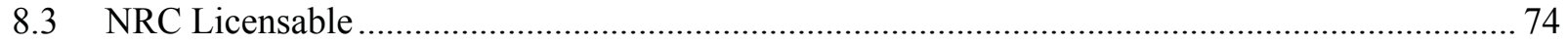

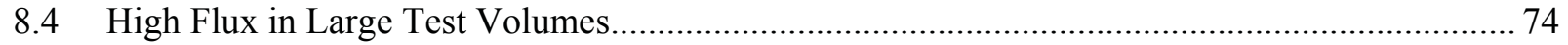

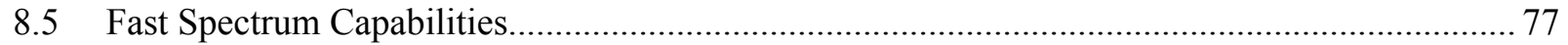

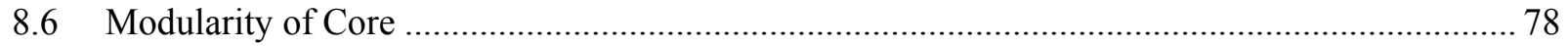

8.7 Online Experiment Access and Rabbits............................................................................... 79

8.8 Minimum waste stream effluents/environmental impact ...........................................................

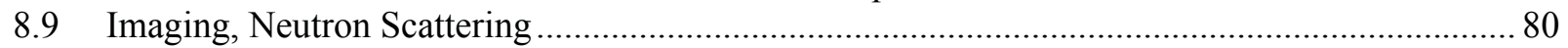

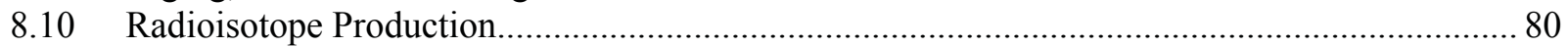

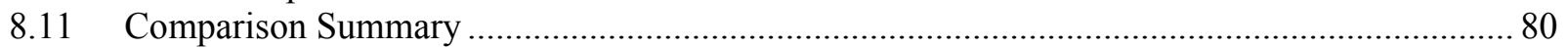

9. Further Design Considerations for Cylindrical Concept ……………………..................... 81

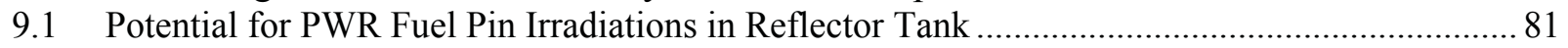

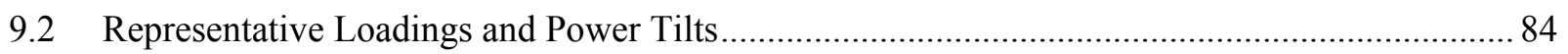

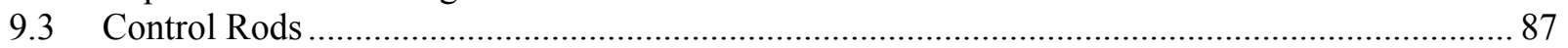

9.4 Potential for Shutdown Rods in Reflector Tank …………………………………………....... 89

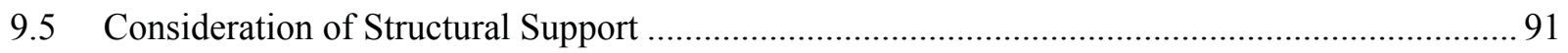

9.6 Test Loop Void Reactivity ............................................................................................ 92 


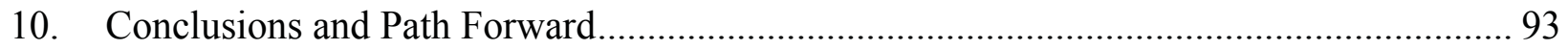

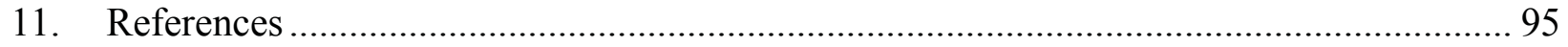

Appendix A Sample Serpent Inputs..................................................................................... 97

Appendix B ATR Core Diagrams....................................................................................... 142

Appendix C Renderings of Cylindrical MATRIX Concept …………………………............. 145

\section{FIGURES}

Figure 2-1. Detailed view of baseline cylindrical core concept (dimensions in $\mathrm{cm}$ ).............................. 9

Figure 2-2. Schematic showing arrangement of fuel plates in cylindrical fuel type............................... 10

Figure 2-3. Diagram of in-pile tubes (IPTs) specified for cylindrical-type core ................................... 10

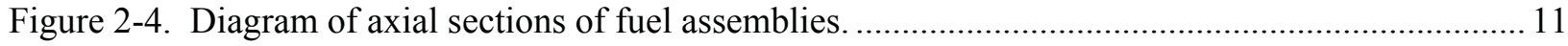

Figure 2-5. Above view of cylindrical concept with reflector tally locations (dimensions in $\mathrm{cm}$ )........... 12

Figure 2-6. Vertical cross section view of cylindrical core concept. .................................................... 13

Figure 2-7. Neutron flux and power map of Cylindrical core model having all fresh fuel...................... 15

Figure 2-8. Cutaway of square fuel assembly with 21 fuel plates (dimensions in $\mathrm{cm}$ ).......................... 18

Figure 2-9. Detailed view of square core concept (dimensions in $\mathrm{cm}$ ) ............................................. 19

Figure 2-10. Above view of square concept with reflector tally locations (dimensions in $\mathrm{cm}$ ). .............20

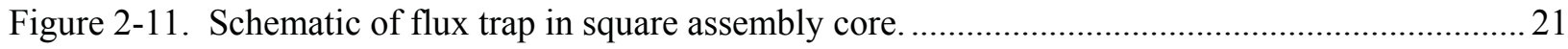

Figure 2-12. Vertical cross section view of square core concept....................................................... 22

Figure 2-13. Neutron flux and power map of Square core model having all fresh fuel...........................24

Figure 2-14. Cutaway of arcuate fuel assembly of the annular concept. ................................................2 27

Figure 2-15. Detailed view of annular core concept (dimensions in $\mathrm{cm}$ ). ..............................................2

Figure 2-16. Schematic of peripheral module of annular design with safety rod. ....................................2 29

Figure 2-17. Schematic of center module of annular design with no safety rod...................................... 30

Figure 2-18. Above view of annular concept with reflector tally locations (dimensions in $\mathrm{cm}$ )..............31

Figure 2-19. Vertical cross section view of annular core concept. ........................................................ 32

Figure 2-20. Neutron flux and power map of Annular core model having all fresh fuel.......................... 34

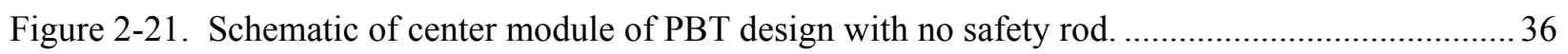

Figure 2-22. Schematic of peripheral module of PBT design with no safety rod.................................... 37

Figure 2-23. Above view of PBT concept with reflector tally locations (dimensions in $\mathrm{cm}$ )................... 38

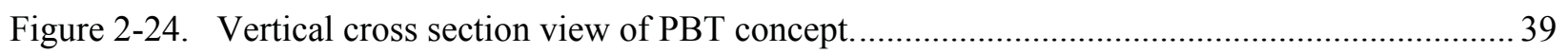

Figure 2-25. Neutron flux map of PBT core model loaded with all fresh fuel. ...................................... 41

Figure 3-1. Minimum wall thickness of candidate RPV materials versus temperature for 1-meter inner diameter. 
Figure 3-2. Minimum wall thickness of candidate PBT materials versus temperature for $28 \mathrm{~cm}$ diameter.

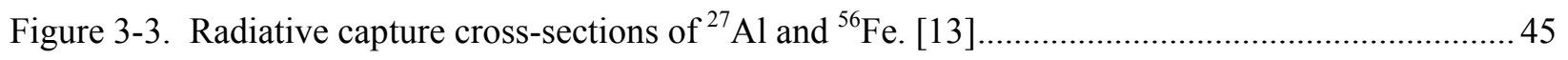

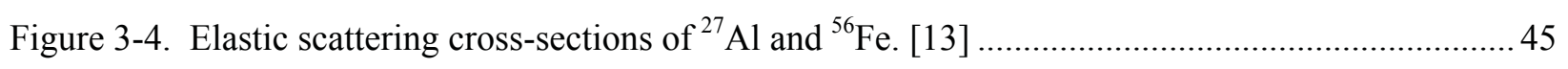

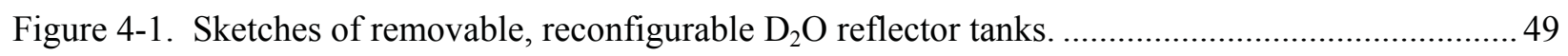

Figure 4-2. Above view of cylindrical core model with $\mathrm{D}_{2} \mathrm{O}$ tanks at axial core center. .......................50

Figure 4-3. Side view of cylindrical core model with $\mathrm{D}_{2} \mathrm{O}$ tanks at axial core center...........................50

Figure 4-4. Above view flux plot of cylindrical core with $\mathrm{D}_{2} \mathrm{O}$ reflector tanks.................................... 51

Figure 4-5. Side view flux plot of cylindrical core with $\mathrm{D}_{2} \mathrm{O}$ reflector tanks. ....................................51

Figure 4-6. Peak thermal flux (at axial centerline) in reflector versus distance from pressure vessel with various reflector tank contents in cylindrical core. .............................................52

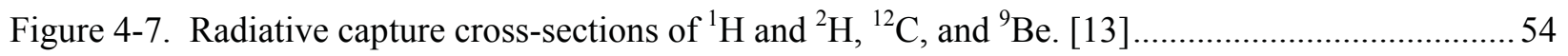

Figure 7-1. Cross-sectional view of the ATR core in the 94-CIC configuration. [16] ............................ 65

Figure 9-1. Reflector PWR pin irradiation hardware dimensions and materials. ................................... 82

Figure 9-2. Baseline Cylindrical core model with PWR irradiation apparatus in reflector...................... 83

Figure 9-3. Assembly layout of prototypic flat 3-batch Cylindrical core. ............................................. 85

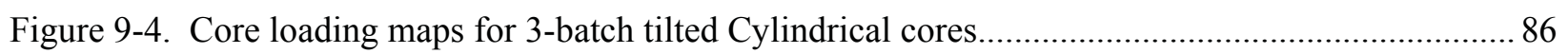

Figure 9-5. Diagram of possible shim rod design inside Cylindrical fuel assembly............................... 88

Figure 9-6. View of Cylindrical core with shutdown blades in $\mathrm{D}_{2} \mathrm{O}$ reflector tank............................. 90

Figure 9-7. Cylindrical fuel element with $0.5 \mathrm{~cm}$ thick side plates and cadmium wires......................... 92

\section{TABLES}

Table 2-1. Parameters of baseline Cylindrical core concept................................................................. 8

Table 2-2. Neutron flux in various locations of baseline Cylindrical core. ............................................. 14

Table 2-3. Parameters of baseline Square core assemblies and core ........................................................ 17

Table 2-4. Neutron flux in various locations of baseline Square core .................................................... 23

Table 2-5. Parameters of baseline annular fuel assemblies and core .......................................................26

Table 2-6. Neutron flux in various locations in the Annular core............................................................ 33

Table 2-7. Parameters of baseline PBT concept fuel assemblies and core ............................................. 35

Table 2-8. Neutron flux in various locations in the PBT core ............................................................. 40

Table 3-1. Maximum allowable stress and minimum wall thickness of candidate pressure

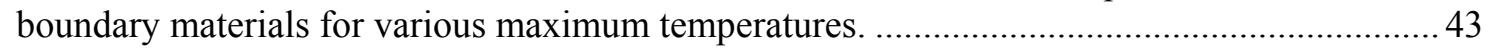

Table 3-2. Results of studies of SS304 pressure boundaries with comparisons to Al-6061 .................... 46

Table 4-1. Reactivity performance of cylindrical core with various reflector contents............................53

Table 5-1. Core rack contents evaluated identified by a Rack ID........................................................... 55 
Table 5-2. Performance parameters for Annular core with varying rack contents.

Table 5-3. Performance parameters for Cylindrical core with varying rack contents.

Table 5-4. Fluence rates in pressure vessels for Annular and Cylindrical cores with various core rack contents.

Table 6-1. Reactivity and flux performance of Annular concepts with $\mathrm{U}_{3} \mathrm{Si}_{2}$ fuel.

Table 6-2. Reactivity and flux performance of Cylindrical concepts with $\mathrm{U}_{3} \mathrm{Si}_{2}$ fuel. 61

Table 6-3. Reactivity and flux performance of baseline Square concept with $\mathrm{U}_{3} \mathrm{Si}_{2}$ fuel 62

Table 6-4. Reactivity and flux performance of baseline PBT concept with $\mathrm{U}_{3} \mathrm{Si}_{2}$ fuel. 62

Table 7-1. Flux values from simplified ATR model depletion calculation. 66

Table 7-2. Fast-to-thermal flux ratios in selected MATRIX cases and ATR model. .68

Table 7-3. Composition of 24" Tall Cylinder of HEU. 70

Table 7-4. Fission rates per gram ${ }^{235} \mathrm{U}$ in prototypic HEU cylinder in ATR and MATRIX. 70

Table 8-1. Summary table of $\mathrm{B}_{1}$ values for MATRIX baseline and variant cases................................... 72

Table 8-2. Reduced field of candidates with alphanumeric identifiers.................................................... 73

Table 8-3. Summary table of average total neutron fluxes and volumes of test spaces............................ 76

Table 8-4. Summary table of peak fast fluxes and volumes of test spaces. .............................................. 78

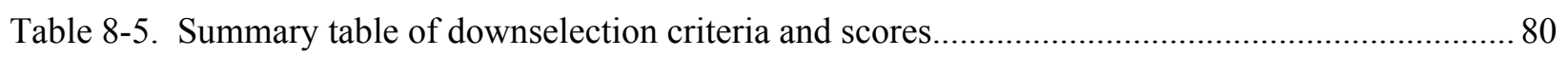

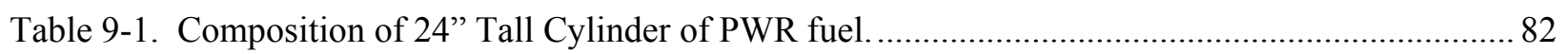

Table 9-2. Reactivity and linear power of PWR fuel pin test in reflector of baseline Cylindrical in core.

Table 9-3. Reactivity and linear power of PWR fuel pin test in reflector of Cylindrical core with SS304 pressure vessel. 84

Table 9-4. Flux in IPTs and power tilt for prototypic flat loading and tilted cores. 87

Table 9-5. Absorber rod worth for various configurations. .89

Table 9-6. Parameters of shutdown blades in reflector tank 90

Table 9-7. Void reactivity results for Cylindrical core with prototypic 3-batch loading. .93 


\section{ACRONYMS}

ASME

ASTM

ATR

BATR

BL

CEA

DOE

FTC

HEU

INEL

INL

LDRD

LEU

MCNP

M-A

M-H

MTC

NE

NR

PBT

pcm

$\mathrm{RJH}$

SS304
American Society of Mechanical Engineers

American Society for Testing of Materials

Advanced Test Reactor

Broad Application Test Reactor

Baseline

Commissariat à l'Energie Atomique

Department of Energy

Fuel Temperature Coefficient

Highly-Enriched Uranium (>20\% by weight)

Idaho National Engineering Laboratory

Idaho National Laboratory

Laboratory-Directed Research and Development

Low-Enriched Uranium ( $<20 \%$ by weight)

Monte Carlo N-Particle code

Multiple-Annular

Modular-Hexagonal

Moderator Temperature Coefficient

Nuclear Energy office within the DOE

Naval Reactors office within the DOE

Pressure Boundary Tube

Percent mille (reactivity unit of $10^{-5}$ )

Réacteur Jules Horowitz (Jules Horowitz Reactor)

Stainless Steel 304 


\section{Evaluation of Concepts for Multiple Application Thermal Reactor for Irradiation eXperiments (MATRIX)}

\section{Background}

\subsection{Introduction}

The Advanced Test Reactor (ATR) is a high power density test reactor designed primarily for accelerated irradiation of nuclear fuels and materials irradiation under controlled environmental conditions. For more than 45 years, the ATR has operated primarily in support of the Office of Naval Reactors (NR) but the mission has gradually expanded to cater to other customers, such as the DOE Office of Nuclear Energy (NE), private industry, and universities. . Featuring nine large flux traps that can accommodate independently-cooled high-pressure In-Pile Tubes (IPTs), ATR is still reliable and effective but increasingly expensive to maintain and operate every year. The sophisticated test hardware and experiments required of recent fuel and materials irradiation campaigns are limited by the safety limits and operating envelope of this aging facility, which are becoming harder to meet as equipment reliability is diminished. Furthermore, the fabrication and transport of its highly enriched uranium (HEU) fuel is deemed a proliferation concern. Under the Global Threat Reduction Initiative (GTRI), ATR and the other high flux test reactors in the US fleet must eventually be converted to low enriched uranium (LEU) in order to continue operating. Studies are underway to assess the costs and technical viability of this fuel conversion. One foreseeable outcome is that the cost of upgrades, fuel conversion, and more frequent LEU fuel loadings needed to keep ATR viable will exceed those of building and operating a replacement reactor. In anticipation of this scenario, work was commenced under the Laboratory Directed Research and Development (LDRD) program to investigate test reactor concepts that could satisfy the current missions of the ATR. Naturally, the needs of other value-adding missions were considered as part of the initial design study.

In the early 1990's, the Idaho National Laboratory (then called Idaho National Engineering Laboratory, or INEL) undertook a similar study aimed at developing and evaluating concepts for a new test reactor. This reactor was envisioned to fulfill a range of customer needs in anticipation of the possible decommissioning of several aging test reactors in the U.S., including the ATR. A cataloguing and ranking of anticipated user needs was performed and a wide variety of reactor concepts were evaluated. The name given to the family of reactors conceptualized was the Broad Application Test Reactor (BATR). [1,2,3,4]

By the end of the BATR study in 1993, two reactor concepts had emerged as leading candidates. Both called for plate fuel and were moderated by beryllium and/or $\mathrm{D}_{2} \mathrm{O}$ and cooled by light water with a rated power of $250 \mathrm{MW}_{\text {th }}$. Several independently cooled IPTs were also called for in both designs. The "Multiple-Annular" (M-A) core featured arcuate fuel assemblies similar to those of the ATR, each surrounding large cylindrical flux traps supporting different loop configurations. The unperturbed spectrum in each large irradiation position could be tailored to user needs through the addition of dedicated reflectors and filters. The "Modular- 
Hexagonal" (M-H) core featured multiple cylindrical fuel assemblies arranged in a hexagonal matrix among an array of uniquely configured flux traps. The entire array of fuel and flux traps was surrounded by a common beryllium primary reflector, a pressure boundary, and a $\mathrm{D}_{2} \mathrm{O}$ secondary reflector. Though each of the two concepts was judged to have advantages and disadvantages, the M-A concept was judged to be slightly superior in part because it had significantly higher fast neutron flux in test locations. The $\mathrm{M}-\mathrm{H}$ core, however, also ranked highly because of its interchangeable grid, which would facilitate core reconfiguration. A third concept, which was also proposed but not analyzed in detail during the BATR project, was an upgraded ATR. This was an evolutionary ATR design calling for several key design changes including a power uprate, a change in coolant direction from downflow to upflow, and an increase in the beryllium reflector size. [1]

In the 1990's, it was assumed that proposed research and test reactors operated by the Department of Energy (DOE) could use highly enriched uranium (HEU) fuel. Because this is no longer considered an option, any new research reactors proposed will likely be required to use low-enriched uranium (LEU) fuel, which contains less than $20 \%{ }^{235} \mathrm{U}$ by mass. This presents a major departure between the current work and the BATR project.

Authorized during fiscal years (FY) 2012 and 2013, this project had the following objectives;

- Update the anticipated customer needs of a new test reactor from the BATR project

- Re-evaluate the BATR concepts of the 1990's and investigate LEU options for them

- Propose modifications to the BATR concepts to better meet the anticipated customer needs

- Analyze and compare the performance of the proposed designs against metrics to be determined in the course of the project

During FY2012, updated user requirements were developed and reported. [5] Also in FY2012, reactor physics analyses were performed verifying the performance of the original BATR concepts with comparisons to LEU versions of these designs. Then, variations on the BATR concepts were introduced and analyzed. [6] From this work, four concepts were identified for further refinement and analysis. This was documented in a separate report, also released at the end of FY2012. [7]

In FY 2013, work continued refining and analyzing the concepts presented in Ref. 7. One change between the FY2012 and FY2013 concepts is a change in core height from $100 \mathrm{~cm}$ to $120 \mathrm{~cm}$. This came as a result of feedback from potential customers regarding the undesirability of having a significantly shorter active core height than that which is currently available in ATR. In addition, a concept having square fuel assembly geometry was introduced as another baseline version of the design. The project also informally adopted the acronym MATRIX (MultipleApplication Thermal Reactor for Irradiation eXperiments).

Section 2 of this report contains descriptions of the four main baseline concepts and their basic performance parameters. Section 3 shows results from comparison of aluminum pressure boundaries to stainless steel. Section 4 gives results from analysis of various reflector contents. Section 5 gives results of analysis of the content of the core rack. Section 6 shows results of comparisons of a fallback fuel meat option. Section 7 discusses comparisons made to an analogous Serpent 2 model of ATR with HEU fuel. Section 8 makes comparisons between the 
reactor concepts and provides some basis for a tentative down-selection to a lead candidate. Section 9 presents further analysis of the down-selected Cylindrical MATRIX design. Finally Conclusions and proposed additional work are given in Section 10.

\subsection{Summary of Customer Requirements}

The needs of the primary ATR customers (NE and NR), gathered through a survey and a meeting held at INL in March of 2012, are [5]:

- High availability/capacity factor $(>70 \%)$

- Must be licensable by the Nuclear Regulatory Commission (NRC)

- High neutron flux (total flux $>10^{15} \mathrm{n} \cdot \mathrm{cm}^{-2} \cdot \mathrm{s}^{-1}$ ) in a large sample volume

- Multiple independently cooled and instrumented IPTs required (variable dimensions)

- Modular type core (re-conformable, flexible) to accommodate changing missions

- Hydraulic shuttle irradiation system (Rabbit)

- Easy access to loops, rabbit tubes, neutron beams, etc.

- Primarily thermal neutron flux spectrum, but some positions capable of fast and epithermal spectra

- Minimum waste stream effluents/environmental impact

- Primary coolant flow in upward direction through the core

The ATR is currently in the conceptual design phase of converting from highly-enriched uranium (HEU) low-enriched uranium (LEU) fuel. The requirements for the converted core are given below [8]:

- An operational cycle length of 56 days at $120 \mathrm{MW}$

- Fast-to-thermal neutron flux ratio within 5\% of current values in a pressurized-water loop test

- Greater than $4.8 \times 10^{14}$ fissions per second per gram ${ }^{235} \mathrm{U}$ in a specimen with 1 gram ${ }^{235} \mathrm{U}$ per linear inch in a standard in-pile tube (SE or SW) operating at $60 \mathrm{MW}$

- $3 / 1$ lobe power split with south corner lobes operating at three times the lobe power of the northern lobes

- Gamma-to-neutron flux ratio within $+10 \% /-0 \%$ of current values

These are written specifically for the converted ATR core and therefore some of them cannot be applied directly to the MATRIX, though the intents of these requirements are considered to be the most important design requirements.

Assuming that the primary mission can be so served, a number of secondary missions were identified for possible, albeit not essential, implementation into the MATRIX design. These include;

- Neutron imaging (e.g. radiography) 
- Neutron scattering and activation experiments

- Radioisotope production (e.g. ${ }^{99} \mathrm{Mo},{ }^{60} \mathrm{Co},{ }^{238} \mathrm{Pu},{ }^{252} \mathrm{Cf}$, etc.)

The spectral and operational needs of these missions vary, placing significant demands on the design of the facility in addition to those listed above. Whether or not a single reactor can serve some or all of these secondary missions will depend on the modularity and configurability of the reactor (the reflector in particular) as well as the functionality of the experimental support systems.

\subsection{Reactor Physics Calculation Methodology}

Reactor physics analyses were performed using the Monte Carlo depletion code Serpent 2 [9]. At a very early conceptual design stage, Monte Carlo simulation tools have clear advantages over most deterministic codes because creating and meshing deterministic models for a wide variety of core geometries is a very labor-intensive process. With modern Monte Carlo tools, geometry definition is efficient and the need to converge the spatial mesh is reduced. Furthermore, cross-section processing is not needed for Monte Carlo analysis since continuous energy cross-sections are used directly. Developed at the VTT Technical Research Center in Finland, Serpent 2 was selected based on the following factors:

- Modern programming - faster than MCNP [10]

- Easy to use, built-in visualization of core model and neutron reactions and flux

- Built-in depletion capable of handling many burnable regions with configurable memory management options

- Automatic fission source development

- Responsive developers

Each case was depleted assuming all fresh fuel at beginning of life (BOL) until $k_{\text {eff }}$ fell below 1.0. Since this is the point at which the reactor is critical, neutron flux tallies were taken from the time step closest to this condition. The number of days of burnup required to reach this point is called $B_{l}$. This value can be used to compare the achievable burnup between reactors and to estimate what the cycle length and discharge burnup would be if other refueling schemes were applied.

\section{Baseline Reactor Concepts}

This section presents descriptions of the four main reactor concepts along with some preliminary analysis. The descriptions in this section are considered baseline cases from which parametric studies are performed in later sections. Those reactor parameters common among all the baseline concepts are:

- Rated power of $250 \mathrm{MW}_{\text {th }}$

- Active core height of $120 \mathrm{~cm}$

- Primary coolant in upflow through the core 
- Primary coolant inlet and outlet temperatures of $52^{\circ} \mathrm{C}$ and $89^{\circ} \mathrm{C}$, respectively

- Nominal primary coolant mass flow rate of $1600 \mathrm{~kg} / \mathrm{s}$

- Plate fuel having coolant channel, fuel meat, and overall plate thicknesses of $2.0 \mathrm{~mm}, 0.5$ $\mathrm{mm}$, and $1.25 \mathrm{~mm}$, respectively

- Primary coolant pressure of $2.3 \mathrm{MPa}$

- Surrounding reflector tank at atmospheric pressure and $27^{\circ} \mathrm{C}$

- Fuel meat temperature of $170^{\circ} \mathrm{C}$

Many of the above parameters are based on the ATR except the last item. Although the ATR is normally operated at less than half its rated thermal power, these studies were performed generally assuming full $250 \mathrm{MW}_{\text {th }}$ power. The addition of a secondary reflector tank (nominally containing $\mathrm{D}_{2} \mathrm{O}$ ) was an idea borrowed from the original BATR work and carried forward to the MATRIX studies. The primary purpose of this feature is ease of integration of secondary missions into the capabilities of the MATRIX reactor. The large atmospheric pressure reflector tank offers space for irradiations, beam tubes, etc. without a high-pressure boundary between the test location and operator access points. Alternatives to this approach are also explored in this work. Descriptions of the four baseline reactor concepts are given in the following subsections.

\subsection{Cylindrical Fuel Concept}

Table 2-1 gives parameters for the "Cylindrical" reactor core, shown in a diagram in Figure 2-1. The Cylindrical concept is so named because the fuel assemblies are constructed as concentric cylinders of plate-type fuel. The core is currently modeled such that each cylindrical element is a single assembly, as is shown schematically in Figure 2-2. However, criticality or other fuel management constraints may dictate that each cylinder be made up of multiple assemblies. For example, three assemblies, each spanning $120^{\circ}$ angle rather than a single element. The 30 fuel assemblies are arranged in a hexagonal array placed in an aluminum core rack having $5 \%$ water by volume for cooling. This rack is surrounded by a pressure vessel, nominally constructed of Al-6064 with stainless steel 304 (SS304) as a fallback alternative. The 
inner radius of the pressure vessel is $44 \mathrm{~cm}$ and it is $4 \mathrm{~cm}$ thick. Outside the pressure vessel is a tank of $\mathrm{D}_{2} \mathrm{O}$ at atmospheric pressure having outer dimensions the same as other variants.

The fuel plates nominally have the same thicknesses as the other concepts and the HEU fuel of the ATR (1.25 mm thick plates with $0.5 \mathrm{~mm}$ thick meat). The baseline fuel form for MATRIX is U-10Mo having uranium density of $15.2 \mathrm{~g} / \mathrm{cm}^{3}$ and Zircaloy cladding. The innermost coolant channel has an inner diameter of $3.95 \mathrm{~cm}$ and the outermost coolant channel has an outer diameter of $10.85 \mathrm{~cm}$. Inside the fuel assembly is a location that can either contain an irradiation test, a control rod or rod follower, or a simple filler to displace coolant water. Unless otherwise specified, this space is modeled as Al-6061. The baseline Cylindrical core is designed to have approximately the same fuel content as the other baseline concepts with approximately $100 \mathrm{~kg}$ of ${ }^{235} \mathrm{U}$ in each. As mentioned previously, all of the baseline cores have an active fueled height of $120 \mathrm{~cm}$, close to the $4 \mathrm{ft}(121.92 \mathrm{~cm})$ height found in ATR. The total mass of uranium in the baseline Cylindrical core is $508 \mathrm{~kg}, 100 \mathrm{~kg}$ of which is ${ }^{235} \mathrm{U}$.

Dispersed among the fuel assemblies are seven IPTs, each corresponding to a flux trap. Figure 2-3 shows a diagram of a flux trap and its associated IPT. Note that Figure 2-2 and Figure 2-3 are not scaled to one another. These are modeled to have similar features as those of the ATR with the sizes of the piping adjusted to fit the size of the flux traps in the Cylindrical design. The outermost layer is an Aluminum baffle. In the ATR, this baffle serves as the structural feature from which fuel elements hang. The same type of fuel assembly support will not be possible in any of the MATRIX designs as currently envisioned. This is because 1) the coolant will be in upflow and simply hanging the elements from a baffle will not be sufficient to keep them in place and 2) the Cylindrical core concept has fuel elements that are not formed around the flux traps as they are in ATR. Nevertheless, some material is included in each of the IPTs here as a placeholder for any structure that would need to be present. These may be removed or modified as design details are added. Inside the baffle are (from outermost to innermost) a water gap, a SS304 insulation tube, a helium gap, the SS304 pressure tube, another water gap, and a SS304 flow tube. Inside this innermost tube is the test space. Assumed for baseline calculations to contain pure Al-6061, this test space has a diameter of $9.3 \mathrm{~cm}$. This is a similar diameter to that of the large NW flux trap in ATR (See Appendix B).

Above and below the fueled portion of the fuel elements are assumed to be regions of plates having no fuel meat and end boxes. Figure 2-4 shows a schematic representation of this. Just above and below the fueled portion of the assembly are homogenized regions of $39 \%$ Zircaloy and $61 \% \mathrm{H}_{2} \mathrm{O}$ coolant with height of $2.0 \mathrm{~cm}$. These percentages come from the assumption that sections of plate not having fuel meat will have the same overall thickness as the fueled portion of the core. Above and below these sections are the top and bottom end boxes. These are assumed to be $20 \mathrm{~cm}$ tall homogenized regions of $80 \% \mathrm{H}_{2} \mathrm{O}$ and $20 \%$ Zircaloy, similar to the representation of end boxes used in ATR modeling. [10]

Figure 2-5 shows an above view of the core, pressure vessel, and the $\mathrm{D}_{2} \mathrm{O}$ reflector tank. Surrounding the pressure vessel, the reflector tank contains $\mathrm{D}_{2} \mathrm{O}$ at atmospheric pressure and near room temperature (assumed to be at $\mathrm{P}=1$ atm and $\mathrm{T}=300 \mathrm{~K}$ ). This feature was envisioned to provide a versatile space of very high thermal flux for irradiation experiments, beam tubes, etc. This feature is compared to other options for reflector tank contents in Section 4 of this report. The numbered reflector tally locations in Figure 2-5 give the distances from core center where flux values are given. These are arranged at an angle of $30^{\circ}$ from the $\mathrm{x}$-axis with distances given in $\mathrm{cm}$. These tally locations will be kept constant among the various reactor concepts discussed 
in this report in order to provide a consistent comparison among their performances. Figure 2-6 shows a vertical cross section of the Cylindrical core design. From this, one can see how the IPTs extend from the bottom to the top of the reflector tank while the active core and fuel assemblies are centered axially in the tank. For neutronic modeling purposes, beyond the outer boundary of the $\mathrm{D}_{2} \mathrm{O}$ tank was considered void.

Table 2-2 shows neutron flux calculated by Serpent 2 for various locations in the core broken down into energy groups. The energy groups are fast $(>1 \mathrm{MeV})$, thermal $(<0.625 \mathrm{eV})$, and total. The tallies were taken in 10 axial segments extending from bottom to top of the 120 $\mathrm{cm}$ tall active core. The center two segments together span $24 \mathrm{~cm}$ of axial height and form the "peak" flux values and the "axially averaged" values are taken as the average over the height of the active core. For the Cylindrical core model, the center and one peripheral flux trap are tallied along with the center of an inner-ring fuel assembly. These calculations were performed assuming $100 \%$ aluminum fill. This gives an estimate of the upper bound of fast neutron flux that is achievable and provides a fair comparison between concepts. If higher thermal neutron flux is desired, this can be moderated accordingly to produce it, though the amount of cooling water in the test location may be constrained by loop void reactivity limits. Therefore, the magnitude of the fast neutron flux in the test locations is used as a measure of the achievable thermal neutron flux in these positions. The four reflector tallies shown in Figure 2-5 are also given in Table 2-2 with the same axial segmentation.

Results from Serpent 2 depletion calculations indicate that the baseline Cylindrical reactor has an initial $k_{\text {eff }}$ of 1.18427 and a $B_{1}$ value of 101 days. The center flux trap has a peak fast and total flux of $3.2 \times 10^{14} \mathrm{n} \cdot \mathrm{cm}^{-2} \cdot \mathrm{s}^{-1}$ and $2.1 \times 10^{15} \mathrm{n} \cdot \mathrm{cm}^{-2} \cdot \mathrm{s}^{-1}$, respectively and the axial flux peaking factor in the center flux trap is 1.3 . The peak thermal flux in the center flux trap is $2.8 \times 10^{14}$ $\mathrm{n} \cdot \mathrm{cm}^{-2} \cdot \mathrm{s}^{-1}$, but as mentioned before, this does not represent the actual peak thermal flux possible in the flux trap. Later studies should provide more insight into the achievable thermal flux. The peripheral flux traps have a peak fast and total flux of $2.8 \times 10^{14} \mathrm{n} \cdot \mathrm{cm}^{-2} \cdot \mathrm{s}^{-1}$ and $1.8 \times 10^{15} \mathrm{n} \cdot \mathrm{cm}^{-2} \cdot \mathrm{s}^{-1}$, respectively. The test spaces inside the inner fuel assemblies have a peak fast and total flux of $5.1 \times 10^{14} \mathrm{n} \cdot \mathrm{cm}^{-2} \cdot \mathrm{s}^{-1}$ and $2.1 \times 10^{15} \mathrm{n} \cdot \mathrm{cm}^{-2} \cdot \mathrm{s}^{-1}$, respectively. Reflector tally position \#1 has a peak thermal neutron flux of $9.8 \times 10^{14} \mathrm{n} \cdot \mathrm{cm}^{-2} \cdot \mathrm{s}^{-1}$.

Figure 2-7 shows a neutron flux map of the Cylindrical core model having all fresh fuel at beginning of depletion. The white areas represent regions of high thermal flux, while blue areas represent low thermal flux. The yellow and red regions represent higher and lower fission power, respectively. Figure 2-7 illustrates how the configuration of this reactor and reflector preserve high fast flux inside the vessel in the IPTs while generating a very large thermal flux outside the pressure vessel in the reflector tank. The flux inside the IPTs can be thermalized up to limits dictated by loop void reactivity. This configuration also permits relatively easy access to a large volume of high thermal neutron flux in the reflector tank without having to penetrate a pressure boundary. This has significant implications with regard to options for using this neutron flux for a variety of missions. 
Table 2-1. Parameters of baseline Cylindrical core concept.

\begin{tabular}{|l|c|}
\hline Parameter & \\
\hline Active Fuel Height $(\mathrm{cm})$ & 120 \\
\hline Fuel Meat Material & $\mathrm{U}-10 \mathrm{Mo}$ \\
\hline${ }^{235} \mathrm{U}$ density $\left(\mathrm{g} / \mathrm{cm}^{3}\right)$ & 3.00 \\
\hline Total U density $\left(\mathrm{g} / \mathrm{cm}^{3}\right)$ & 15.2 \\
\hline Enrichment $(\mathrm{wt} . \%)$ & 19.7 \\
\hline Number of Assemblies & 24 \\
\hline Fuel Plates Per Assembly & 10 \\
\hline Inner diameter of innermost coolant channel $(\mathrm{cm})$ & 3.95 \\
\hline Outer diameter of outermost coolant channel $(\mathrm{cm})$ & 10.85 \\
\hline Width of coolant channels between fuel plates $(\mathrm{cm})$ & 0.2 \\
\hline Fuel plate thickness $(\mathrm{cm})$ & 0.125 \\
\hline Fuel meat thickness $(\mathrm{cm})$ & 0.05 \\
\hline Cladding Composition & Zircaloy-4 \\
\hline Central Cylinder Composition & Zircaloy-4 \\
\hline Total ${ }^{235} \mathrm{U}$ per assembly $(\mathrm{kg})$ & 4.2 \\
\hline Total ${ }^{238} \mathrm{U}$ per assembly $(\mathrm{kg})$ & 17.0 \\
\hline Total ${ }^{235} \mathrm{U}$ in core $(\mathrm{kg})$ & 100 \\
\hline Total ${ }^{238} \mathrm{U}$ in core $(\mathrm{kg})$ & 408 \\
\hline
\end{tabular}




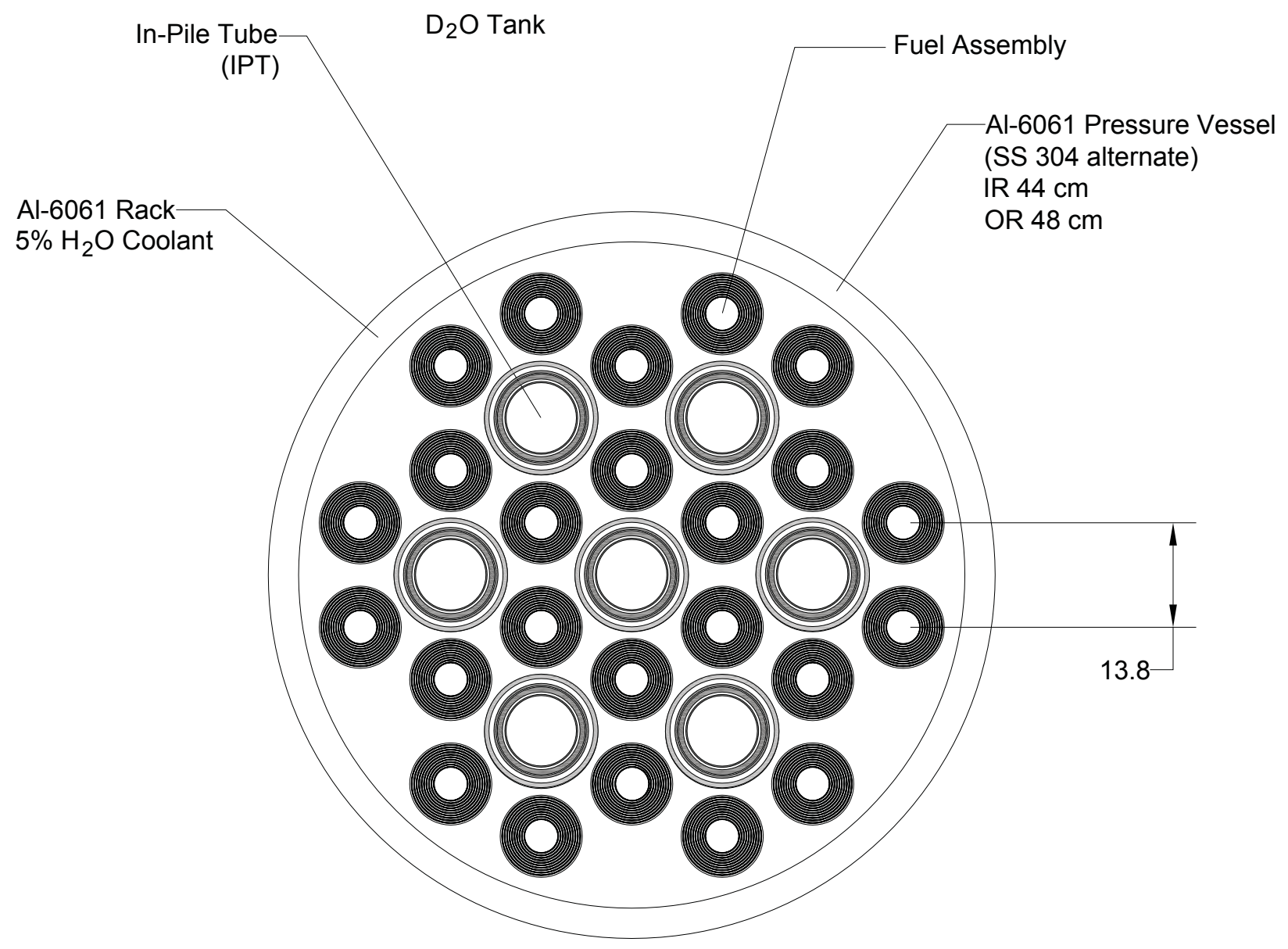

Figure 2-1. Detailed view of baseline cylindrical core concept (dimensions in $\mathrm{cm}$ ). 


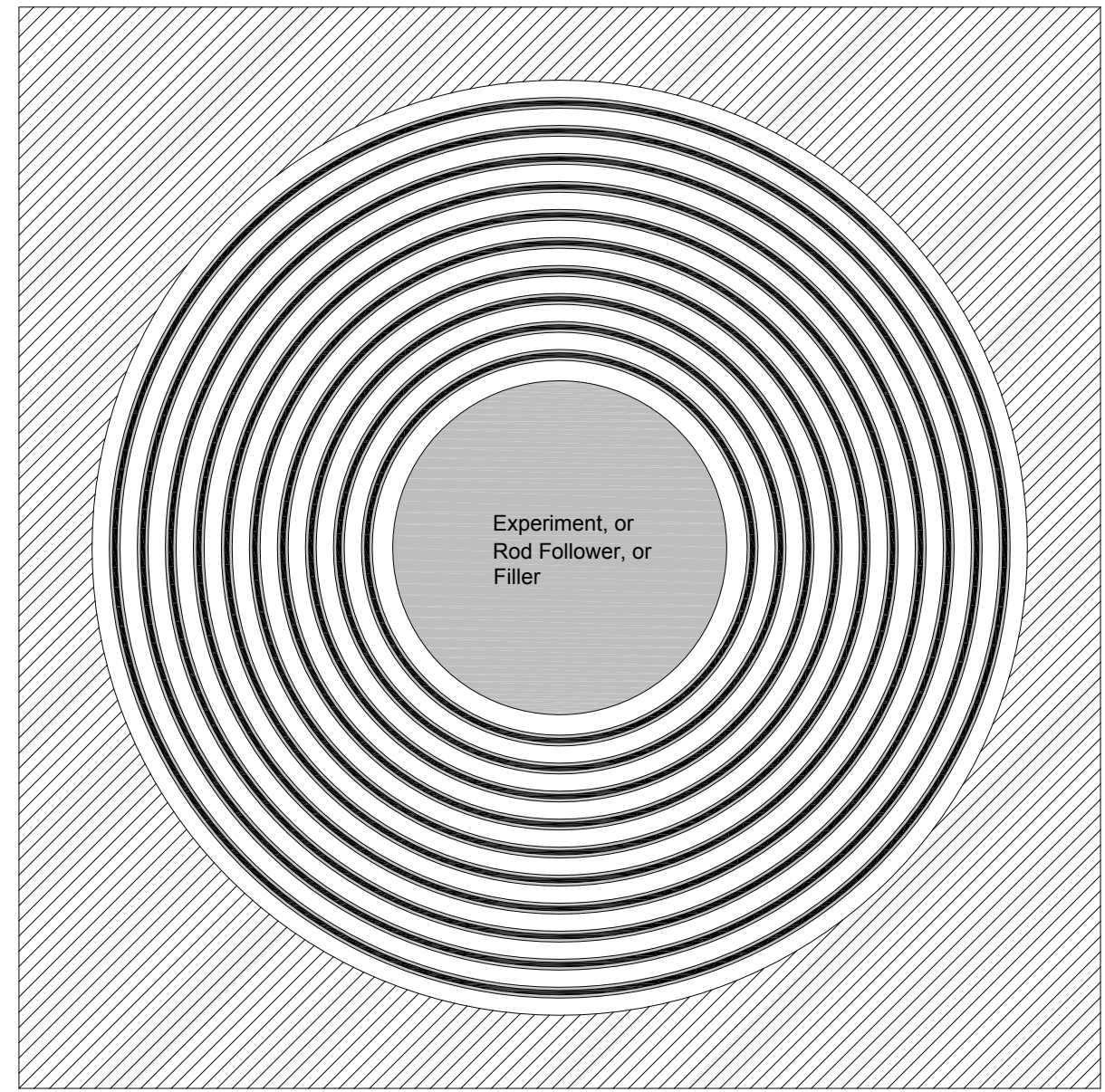

Figure 2-2. Schematic showing arrangement of fuel plates in cylindrical fuel type.

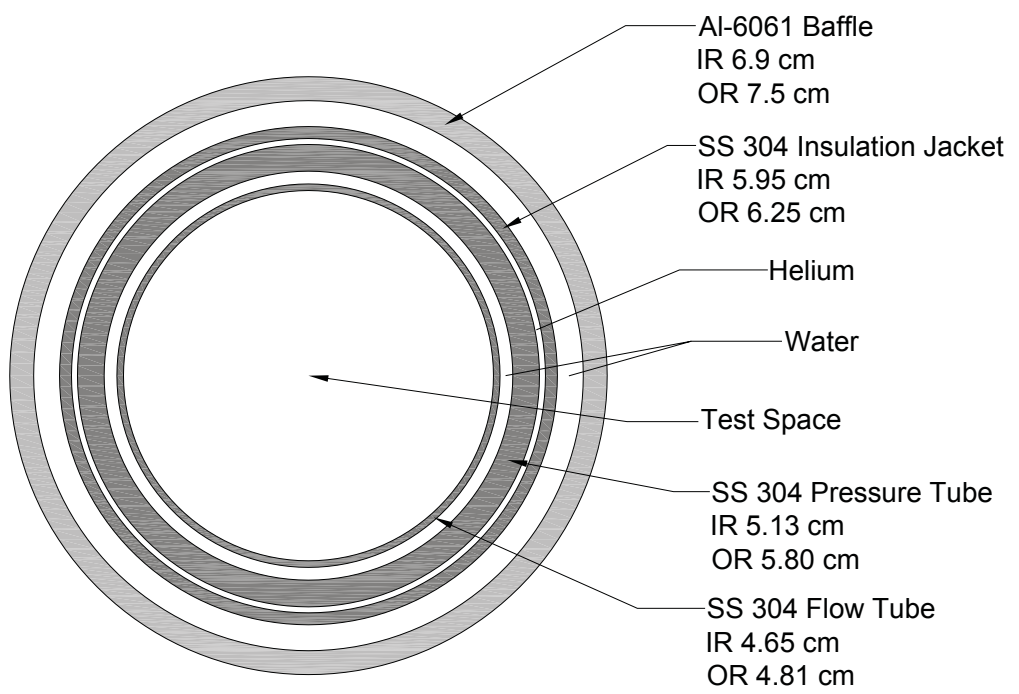

Figure 2-3. Diagram of in-pile tubes (IPTs) specified for cylindrical-type core. 


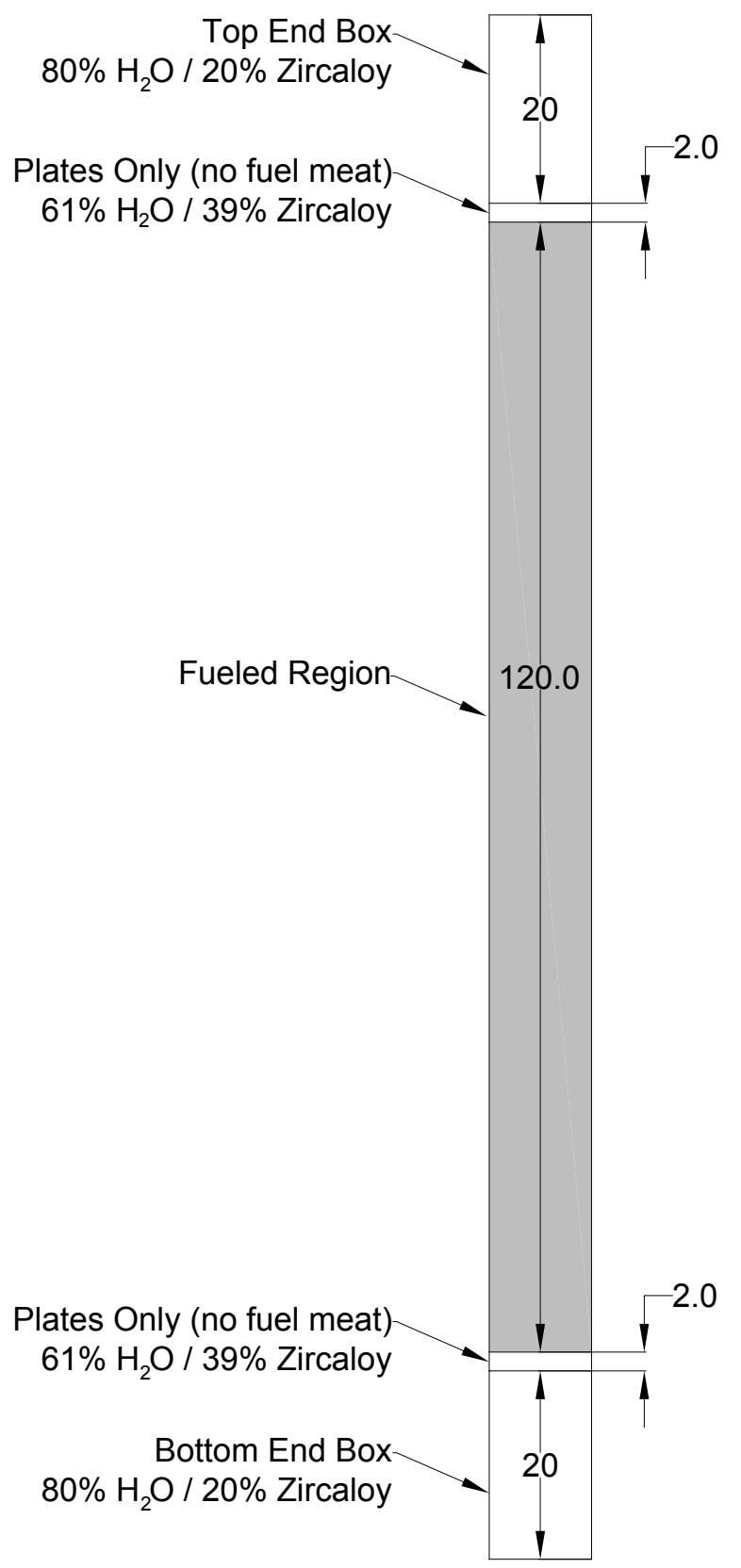

Figure 2-4. Diagram of axial sections of fuel assemblies. 


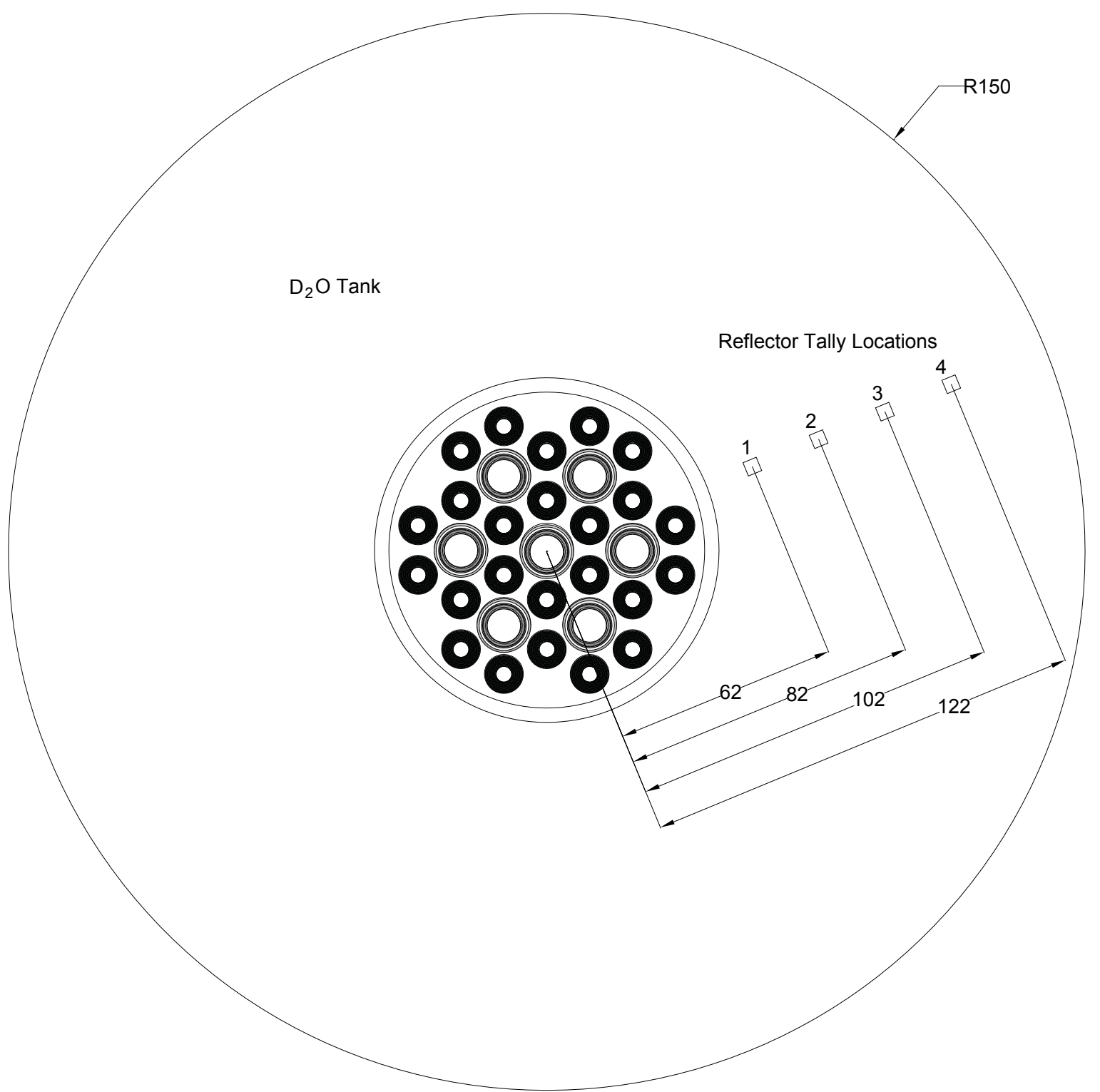

Figure 2-5. Above view of cylindrical concept with reflector tally locations (dimensions in $\mathrm{cm}$ ). 

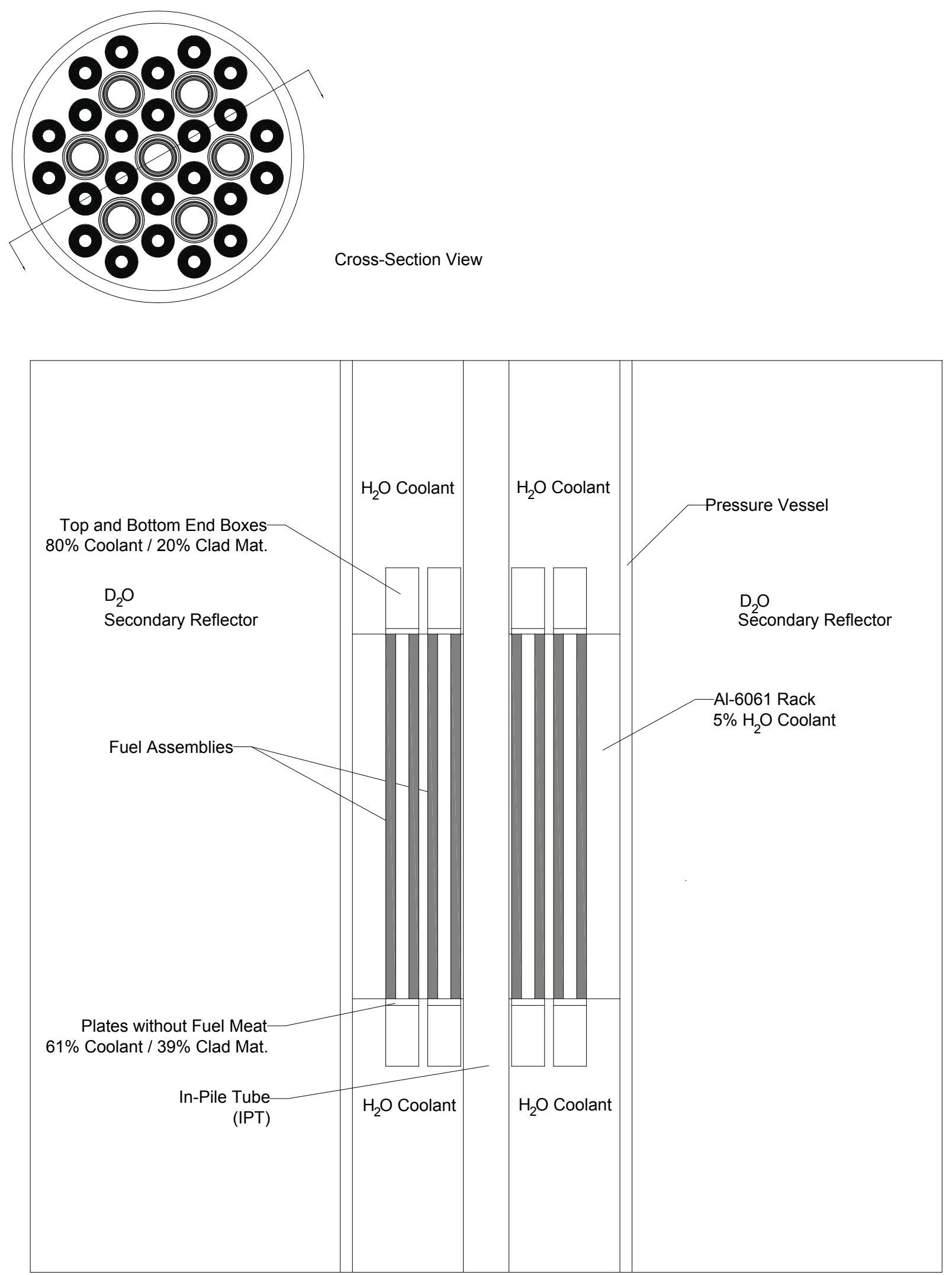

Figure 2-6. Vertical cross section view of cylindrical core concept. 
Table 2-2. Neutron flux in various locations of baseline Cylindrical core. ${ }^{a}$

\begin{tabular}{|c|c|c|c|}
\hline \multirow[t]{2}{*}{ Tally Location } & \multicolumn{3}{|c|}{$\begin{array}{c}\text { Neutron Flux in Reflector }\left(\mathrm{n} \cdot \mathrm{cm}^{-2} \mathrm{~s}^{-1}\right) \\
\text { Fast Flux }(>1 \mathrm{MeV}) \\
\text { Thermal Flux }(<0.625 \mathrm{eV}) \\
\text { Total Flux }\end{array}$} \\
\hline & Peak & $\begin{array}{c}\text { Axially } \\
\text { Averaged }\end{array}$ & $\begin{array}{c}\text { Peak/ } \\
\text { Average }\end{array}$ \\
\hline \multirow{3}{*}{ Center Flux Trap } & $3.22 \mathrm{E}+14$ & $2.42 \mathrm{E}+14$ & 1.33 \\
\hline & $2.77 \mathrm{E}+14$ & $2.12 \mathrm{E}+14$ & 1.31 \\
\hline & $2.07 \mathrm{E}+15$ & $1.55 \mathrm{E}+15$ & 1.33 \\
\hline \multirow{3}{*}{ Peripheral Flux Trap } & $2.79 \mathrm{E}+14$ & $2.11 \mathrm{E}+14$ & 1.32 \\
\hline & $2.42 \mathrm{E}+14$ & $1.87 \mathrm{E}+14$ & 1.29 \\
\hline & $1.80 \mathrm{E}+15$ & $1.36 \mathrm{E}+15$ & 1.32 \\
\hline \multirow{3}{*}{ Inner Fuel Assembly } & $5.07 \mathrm{E}+14$ & $3.86 \mathrm{E}+14$ & 1.31 \\
\hline & $1.42 \mathrm{E}+14$ & $1.12 \mathrm{E}+14$ & 1.28 \\
\hline & $2.14 \mathrm{E}+15$ & $1.62 \mathrm{E}+15$ & 1.32 \\
\hline \multirow{3}{*}{ Reflector Tally 1} & $5.52 \mathrm{E}+12$ & $4.27 \mathrm{E}+12$ & 1.29 \\
\hline & $9.82 \mathrm{E}+14$ & $7.96 \mathrm{E}+14$ & 1.23 \\
\hline & $1.14 \mathrm{E}+15$ & $9.20 \mathrm{E}+14$ & 1.24 \\
\hline \multirow{3}{*}{ Reflector Tally 2} & - & - & - \\
\hline & $7.13 \mathrm{E}+14$ & $5.96 \mathrm{E}+14$ & 1.20 \\
\hline & $7.22 \mathrm{E}+14$ & $6.03 \mathrm{E}+14$ & 1.20 \\
\hline \multirow{3}{*}{ Reflector Tally 3} & - & - & - \\
\hline & $4.18 \mathrm{E}+14$ & $3.58 \mathrm{E}+14$ & 1.17 \\
\hline & $4.19 \mathrm{E}+14$ & $3.59 \mathrm{E}+14$ & 1.17 \\
\hline \multirow{3}{*}{ Reflector Tally 4} & - & - & - \\
\hline & $2.12 \mathrm{E}+14$ & $1.85 \mathrm{E}+14$ & 1.15 \\
\hline & $2.12 \mathrm{E}+14$ & $1.85 \mathrm{E}+14$ & 1.15 \\
\hline
\end{tabular}

${ }^{\mathrm{a}}$ Initial $k_{\text {eff }}=1.18427, \mathrm{~B}_{1}=100$ days 


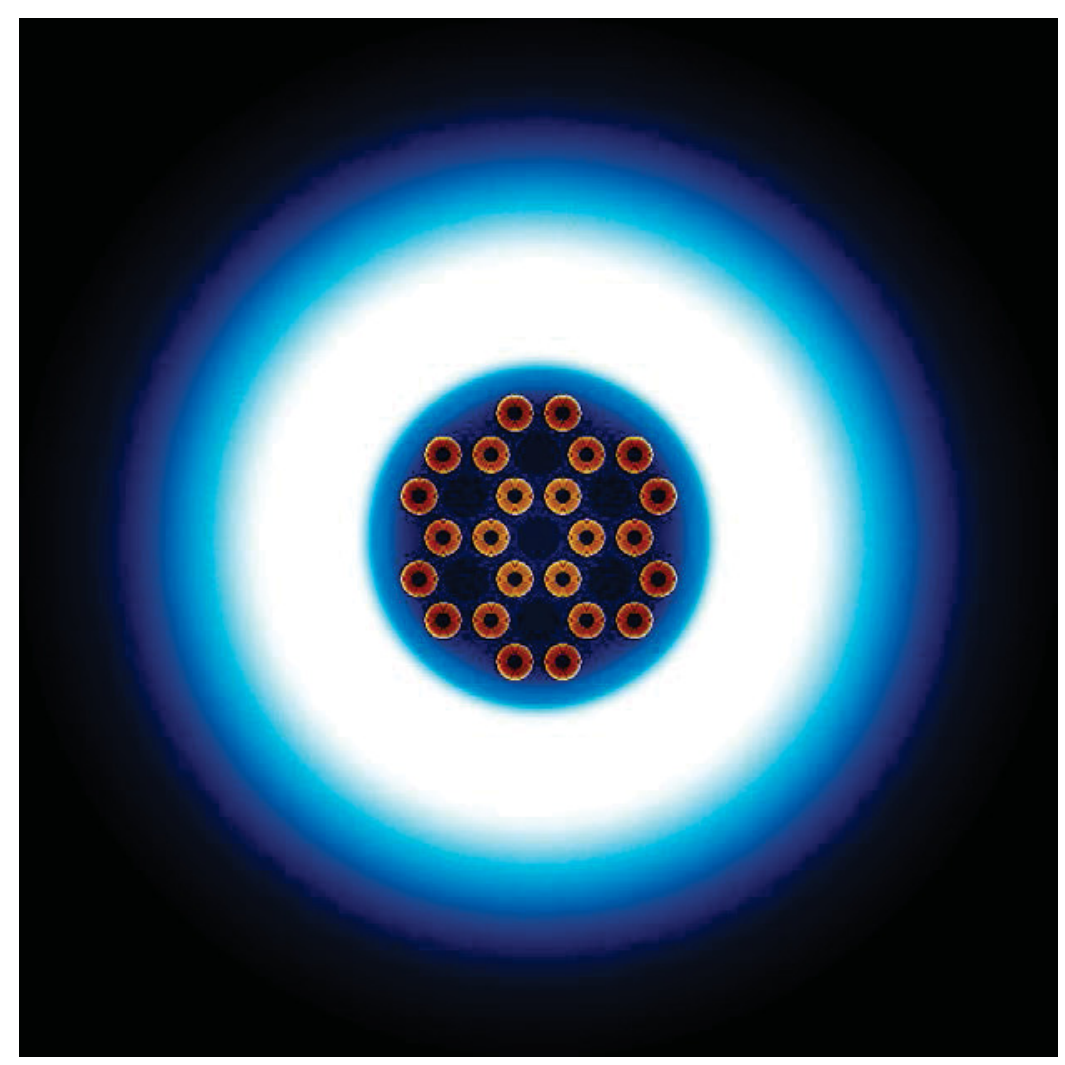

Figure 2-7. Neutron flux and power map of Cylindrical core model having all fresh fuel.

\subsection{Square Concept}

The fuel assemblies of the Cylindrical core model have a minimum plate diameter of approximately $4 \mathrm{~cm}$. There is some uncertainty as to whether or not plates with this curvature can be fabricated from cladded U10Mo . Therefore, an alternative fuel assembly concept was proposed having similar features as the Cylindrical core (e.g. single aluminum vessel surrounding aluminum core rack) but with square fuel assemblies. Henceforth referred to as the "Square" core, this concept uses 36 square assemblies with $7.5 \mathrm{~cm}$ sides. 
Table 2-3 shows the main parameters of the Square core concept. A cross-section view of one of the assemblies is shown in Figure 2-8. The wall thicknesses of the outer can of the assemblies are $0.2375 \mathrm{~cm}$ thick and the axial top and bottom of the assemblies have the same homogenized regions described for the Cylindrical core and depicted in Figure 2-4. There are 21 plates per fuel assembly and fuel cladding and the outer can of the assemblies are Zircaloy.

The core height and the design of the IPTs and flux traps are the same as the Cylindrical core. Figure 2-9 shows an above view of the general core layout. As with all of the baseline cores, the fuel is U-10Mo having uranium density of $15.2 \mathrm{~g} / \mathrm{cm}^{3}$. Fuel plate thickness and fuel meat thickness is the same between all baseline core models. The Square core was designed to have roughly the same amount of fuel as the Cylindrical core with $485 \mathrm{~kg}$ of uranium. For initial analysis, the outer boundaries of the assembly cans are assumed to just touch. In a final design, allowance would have to be made for cooling between cans and room for expansion. However, this is neglected for simplification of scoping calculations.

The assemblies are arranged in a square array with 7 IPTs displacing four lattice positions each. Additional test positions and locations for shim and or shutdown rods are also included as placeholders. The pressure vessel was expanded slightly from the Cylindrical core to accommodate the arrangement of fuel assemblies, test spaces, and control spaces. The inner diameter of the vessel is $47 \mathrm{~cm}$ and the thickness remains $4 \mathrm{~cm}$. Figure 2-10 shows an above view of the core, pressure vessel, and the $\mathrm{D}_{2} \mathrm{O}$ reflector tank. Just as in the Cylindrical core, the reflector tank contains $\mathrm{D}_{2} \mathrm{O}$ at atmospheric pressure and near room temperature (again assumed to be at $\mathrm{P}=1 \mathrm{~atm}$ and $\mathrm{T}=300 \mathrm{~K}$ ). The numbered reflector tallies are located at the same distances from core center as in the Cyindrical core. Figure 2-11 shows a diagram of the IPTs surrounding a flux trap in the Square core. Again, the available test space has a diameter of approximately 9 $\mathrm{cm}$.

Figure 2-12 shows a vertical cross section view of square core concept. Because a center cut-away as shown in the upper left corner of the figure is between assemblies, detail is not shown in the core sections. Note that the assemblies have homogenized regions extending above and below the $120 \mathrm{~cm}$ active core region as was the case in the Cylindrical core. Again, the IPTs extend the entire height of the $3 \mathrm{~m}$ tall $\mathrm{D}_{2} \mathrm{O}$ tank.

Results from Serpent depletion calculations indicate that the baseline Square reactor has an initial $k_{\text {eff }}$ of 1.14066 and a $B_{1}$ of 70 days. Table 2-4 shows neutron flux in various locations of baseline Square core. The center flux trap has a peak fast and total flux of $2.7 \times 10^{14} \mathrm{n} \cdot \mathrm{cm}^{-2} \cdot \mathrm{s}^{-1}$ and $2.0 \times 10^{15} \mathrm{n} \cdot \mathrm{cm}^{-2} \cdot \mathrm{s}^{-1}$, respectively and the axial flux peaking factor in the center flux trap is 1.3. The peripheral flux traps are not identical in this core. The peak fast flux values in the peripheral flux traps range from $2.4 \times 10^{14} \mathrm{n} \cdot \mathrm{cm}^{-2} \cdot \mathrm{s}^{-1}$ to $2.7 \times 10^{14} \mathrm{n} \cdot \mathrm{cm}^{-2} \cdot \mathrm{s}^{-1}$. The peak total flux values in the peripheral flux traps range from $1.6 \times 10^{15} \mathrm{n} \cdot \mathrm{cm}^{-2} \cdot \mathrm{s}^{-1}$ to $1.8 \times 10^{15} \mathrm{n} \cdot \mathrm{cm}^{-2} \cdot \mathrm{s}^{-1}$. Reflector tally position \#1 has a peak thermal neutron flux of $1.1 \times 10^{15} \mathrm{n} \cdot \mathrm{cm}^{-2} \cdot \mathrm{s}^{-1}$. Figure 2-13 shows a neutron flux map of the all-fresh core at beginning of irradiation. 
Table 2-3. Parameters of baseline Square core assemblies and core

\begin{tabular}{|l|c|}
\hline Parameter & \\
\hline Active Fuel Height $(\mathrm{cm})$ & 120 \\
\hline Fuel Meat Material & U-10Mo \\
\hline${ }^{235} \mathrm{U}$ density $\left(\mathrm{g} / \mathrm{cm}^{3}\right)$ & 3.00 \\
\hline Total U density $\left(\mathrm{g} / \mathrm{cm}^{3}\right)$ & 15.2 \\
\hline Enrichment $(\mathrm{wt} . \%)$ & 19.7 \\
\hline Number of Assemblies & 36 \\
\hline Square Assembly Width $(\mathrm{cm})$ & 7.5 \\
\hline Fuel Plates Per Assembly & 21 \\
\hline Width of coolant channels between fuel plates $(\mathrm{cm})$ & 0.2 \\
\hline Fuel plate thickness $(\mathrm{cm})$ & 0.125 \\
\hline Fuel meat thickness $(\mathrm{cm})$ & 0.05 \\
\hline Cladding Composition & Zircaloy-4 \\
\hline Assembly Wall Composition & Zircaloy-4 \\
\hline Assembly Wall Plate Thickness (cm) & 0.2375 \\
\hline Total ${ }^{235} \mathrm{U}$ per assembly (kg) & 2.66 \\
\hline Total ${ }^{238} \mathrm{U}$ per assembly (kg) & 10.8 \\
\hline Total ${ }^{235} \mathrm{U}$ in core (kg) & 95.6 \\
\hline Total ${ }^{238} \mathrm{U}$ in core $(\mathrm{kg})$ & 389 \\
\hline
\end{tabular}




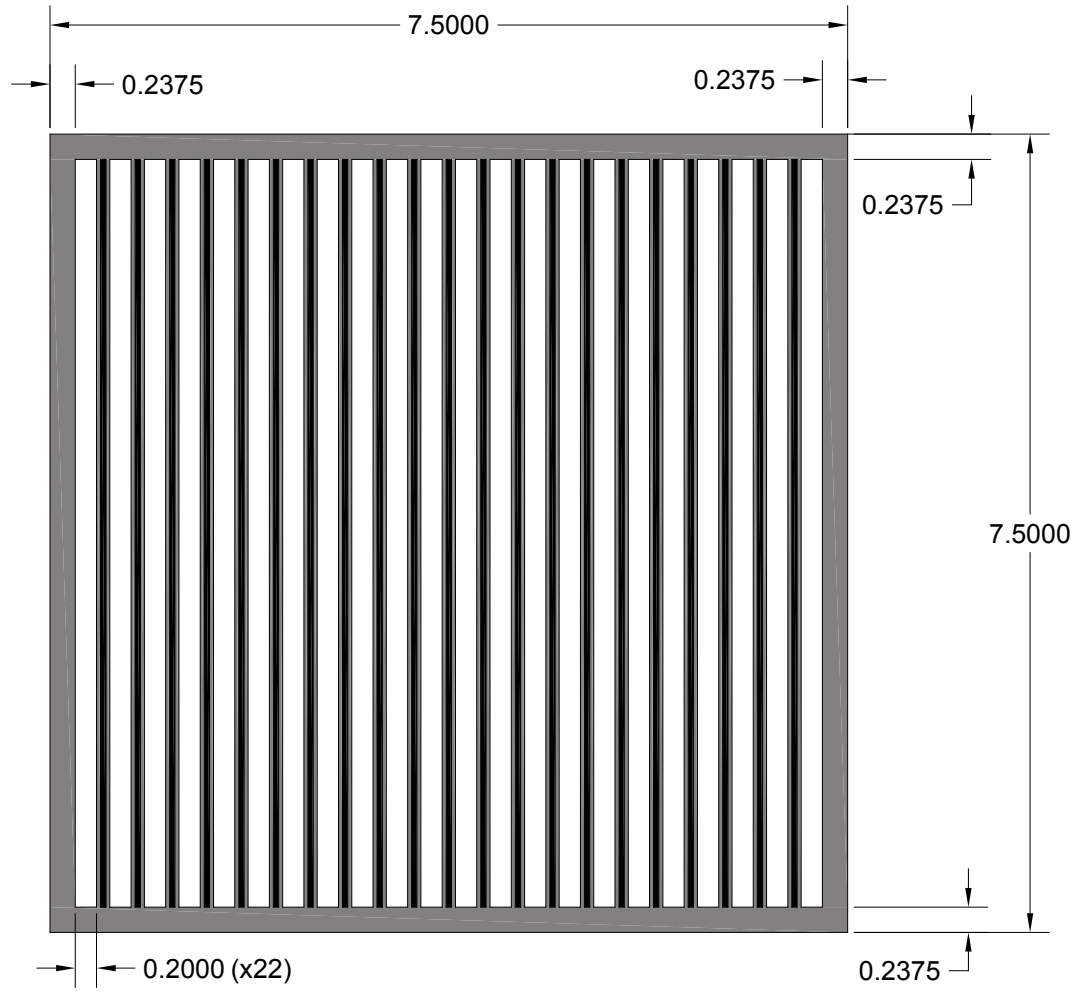

Figure 2-8. Cutaway of square fuel assembly with 21 fuel plates (dimensions in $\mathrm{cm}$ ). 


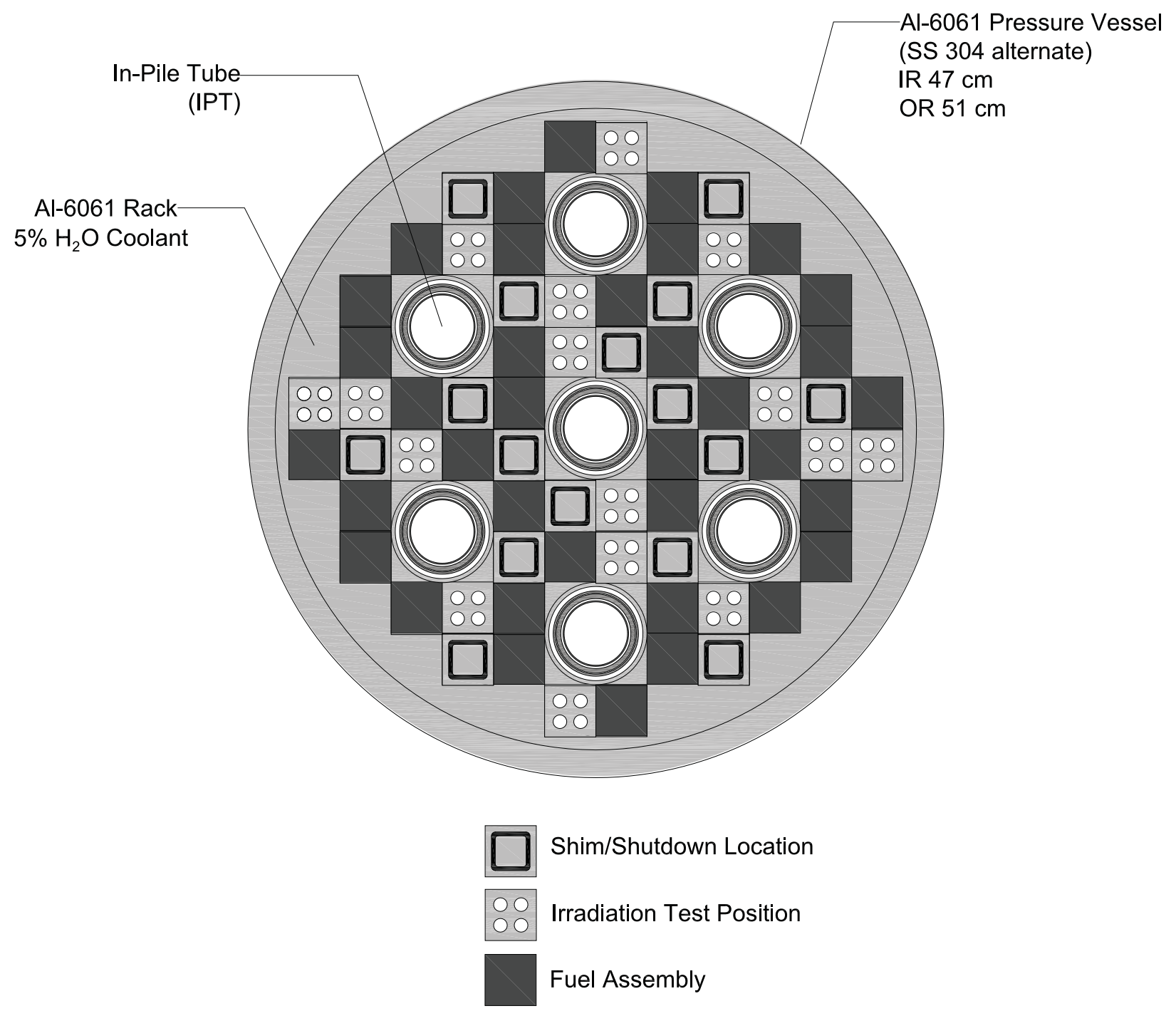

Figure 2-9. Detailed view of square core concept (dimensions in $\mathrm{cm}$ ). 


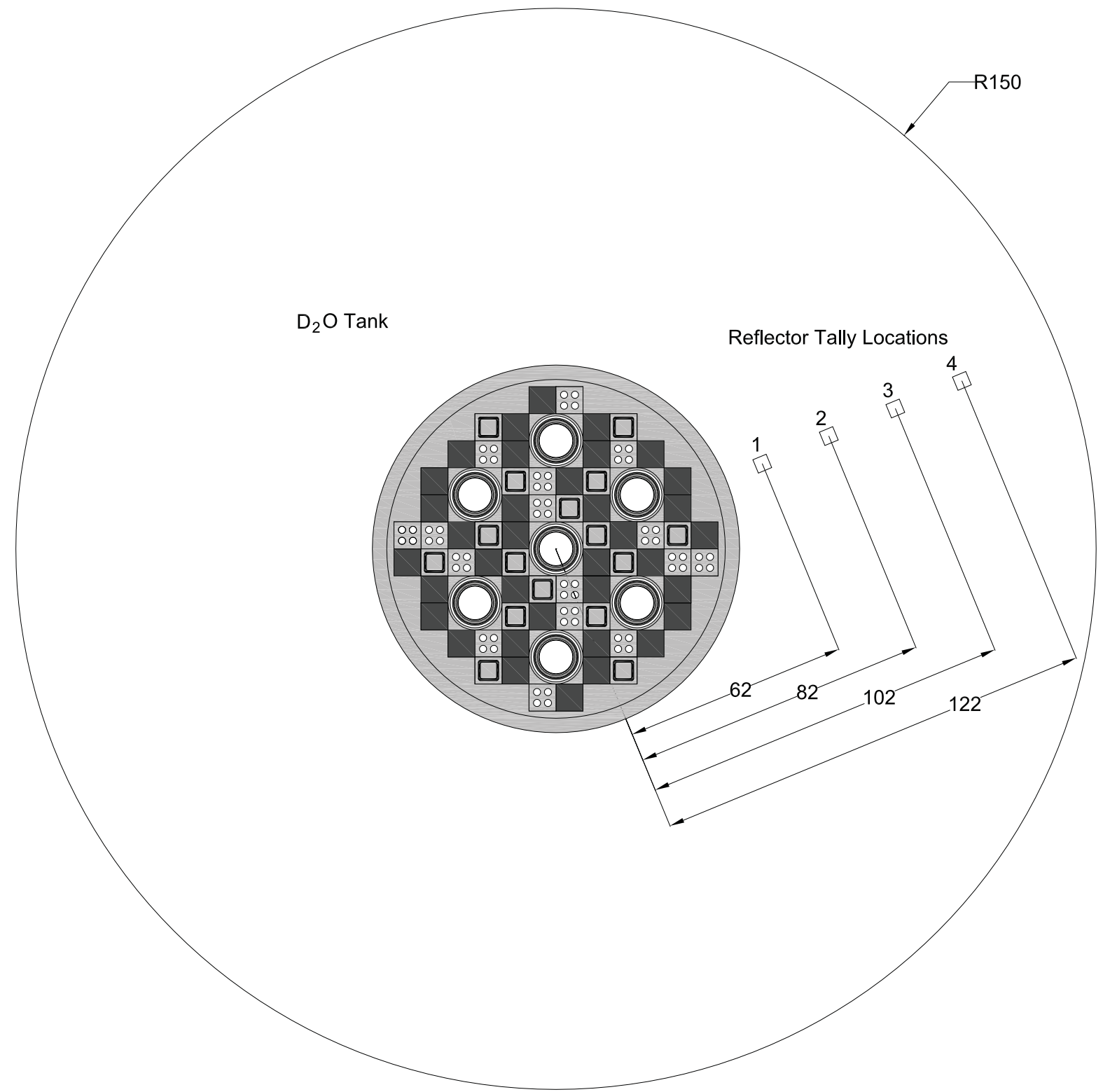

Figure 2-10. Above view of square concept with reflector tally locations (dimensions in $\mathrm{cm}$ ). 


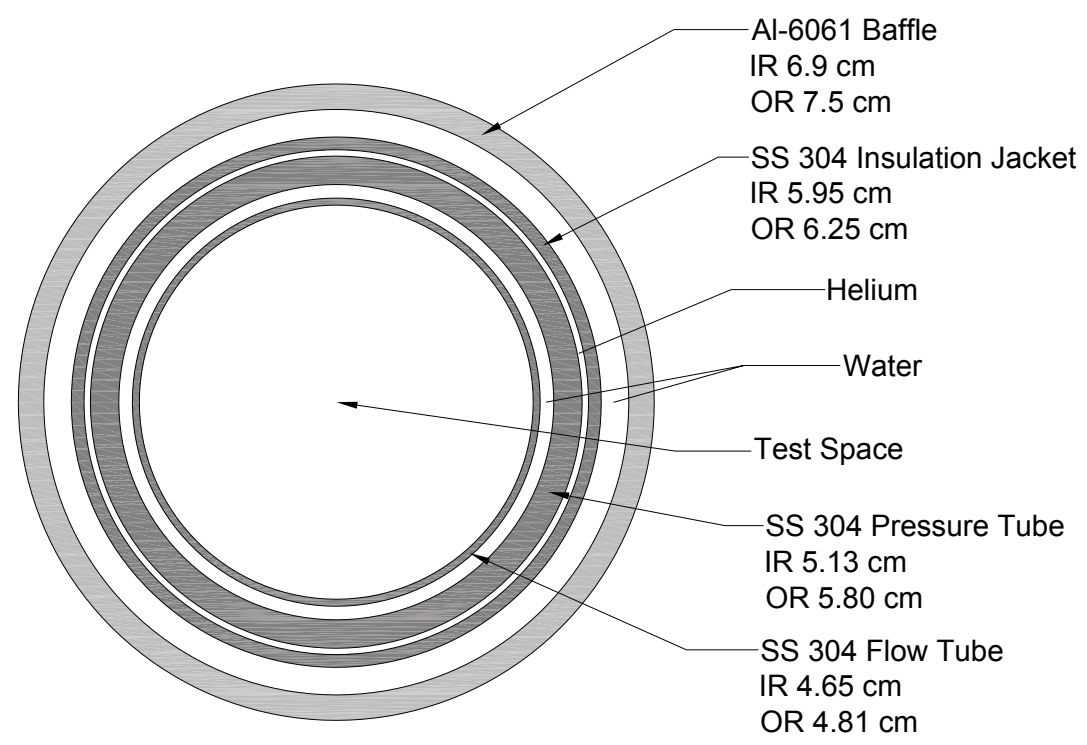

Figure 2-11. Schematic of flux trap in square assembly core. 

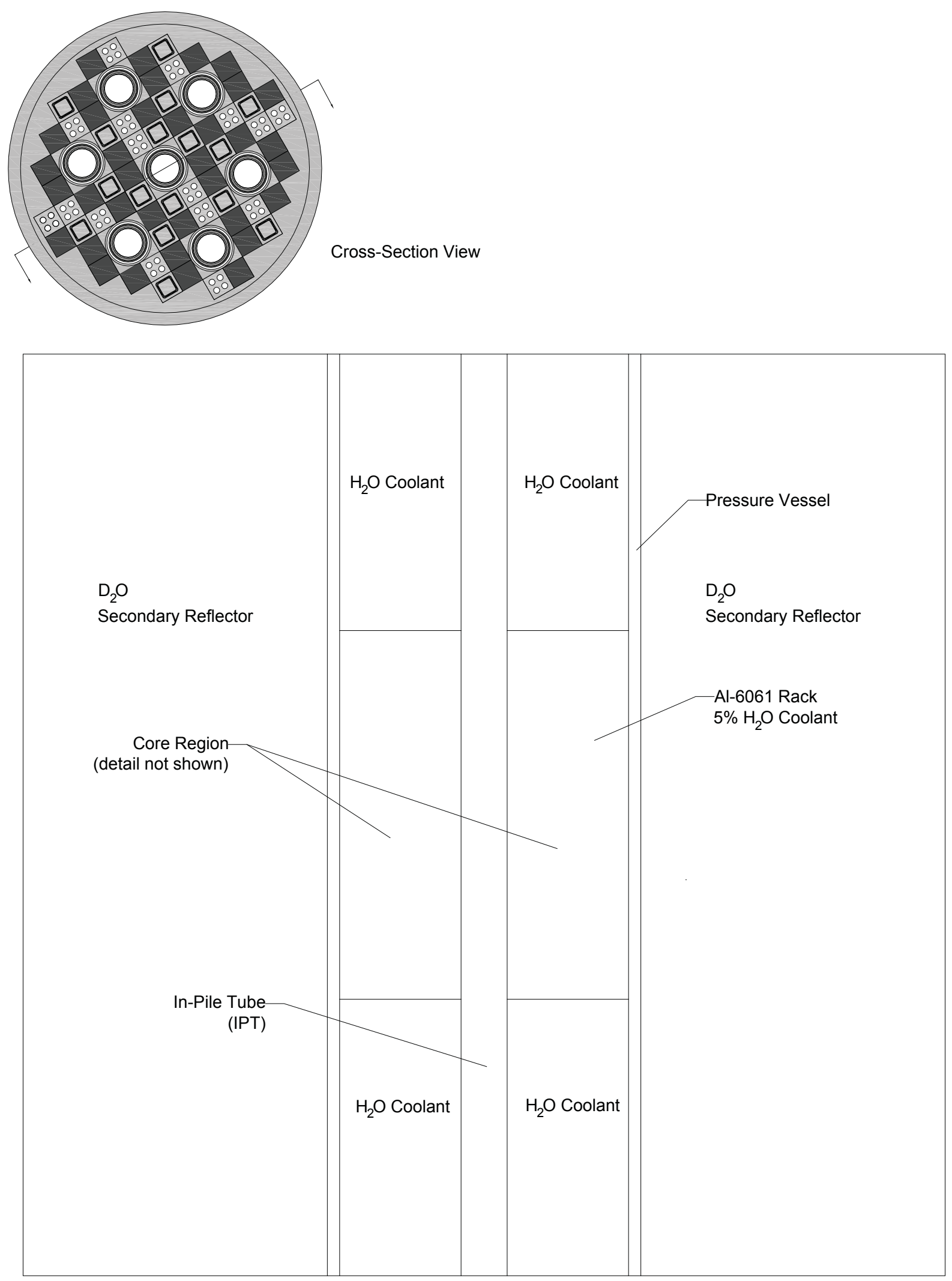

Figure 2-12. Vertical cross section view of square core concept. 
Table 2-4. Neutron flux in various locations of baseline Square core. ${ }^{\mathrm{a}}$

\begin{tabular}{|c|c|c|c|}
\hline \multirow[t]{2}{*}{ Tally Location } & \multicolumn{3}{|c|}{$\begin{array}{c}\text { Neutron Flux in Reflector }\left(\mathrm{n} \cdot \mathrm{cm}^{-2} \mathrm{~s}^{-1}\right) \\
\text { Fast Flux }(>1 \mathrm{MeV}) \\
\text { Thermal Flux }(<0.625 \mathrm{eV}) \\
\text { Total Flux }\end{array}$} \\
\hline & Peak & $\begin{array}{c}\text { Axially } \\
\text { Averaged }\end{array}$ & $\begin{array}{c}\text { Peak/ } \\
\text { Average }\end{array}$ \\
\hline \multirow{3}{*}{ Center Flux Trap } & $2.71 \mathrm{E}+14$ & $2.06 \mathrm{E}+14$ & 1.32 \\
\hline & $2.84 \mathrm{E}+14$ & $2.19 \mathrm{E}+14$ & 1.30 \\
\hline & $2.00 \mathrm{E}+15$ & $1.52 \mathrm{E}+15$ & 1.32 \\
\hline \multirow{3}{*}{ NE Flux Trap } & $2.68 \mathrm{E}+14$ & $2.04 \mathrm{E}+14$ & 1.32 \\
\hline & $2.34 \mathrm{E}+14$ & $1.82 \mathrm{E}+14$ & 1.29 \\
\hline & $1.79 \mathrm{E}+15$ & $1.36 \mathrm{E}+15$ & 1.32 \\
\hline \multirow{3}{*}{ N Flux Trap } & $2.43 \mathrm{E}+14$ & $1.85 \mathrm{E}+14$ & 1.31 \\
\hline & $2.18 \mathrm{E}+14$ & $1.69 \mathrm{E}+14$ & 1.29 \\
\hline & $1.62 \mathrm{E}+15$ & $1.23 \mathrm{E}+15$ & 1.32 \\
\hline \multirow{3}{*}{ NW Flux Trap } & $2.70 \mathrm{E}+14$ & $2.05 \mathrm{E}+14$ & 1.32 \\
\hline & $2.37 \mathrm{E}+14$ & $1.83 \mathrm{E}+14$ & 1.30 \\
\hline & $1.80 \mathrm{E}+15$ & $1.37 \mathrm{E}+15$ & 1.32 \\
\hline \multirow{3}{*}{ Reflector Tally 1} & $6.83 \mathrm{E}+12$ & $5.64 \mathrm{E}+12$ & 1.21 \\
\hline & $9.09 \mathrm{E}+14$ & $7.36 \mathrm{E}+14$ & 1.24 \\
\hline & $1.12 \mathrm{E}+15$ & $8.99 \mathrm{E}+14$ & 1.25 \\
\hline \multirow{3}{*}{ Reflector Tally 2} & - & - & - \\
\hline & $7.17 \mathrm{E}+14$ & $5.95 \mathrm{E}+14$ & 1.21 \\
\hline & $7.30 \mathrm{E}+14$ & $6.05 \mathrm{E}+14$ & 1.21 \\
\hline \multirow{3}{*}{ Reflector Tally 3} & - & - & - \\
\hline & $4.23 \mathrm{E}+14$ & $3.58 \mathrm{E}+14$ & 1.18 \\
\hline & $4.23 \mathrm{E}+14$ & $3.58 \mathrm{E}+14$ & 1.18 \\
\hline \multirow{3}{*}{ Reflector Tally 4} & - & - & - \\
\hline & $2.10 \mathrm{E}+14$ & $1.83 \mathrm{E}+14$ & 1.14 \\
\hline & $2.10 \mathrm{E}+14$ & $1.83 \mathrm{E}+14$ & 1.14 \\
\hline
\end{tabular}

\footnotetext{
${ }^{\mathrm{a}}$ Initial $k_{\text {eff }}=1.14066, \mathrm{~B}_{1}=70$ days
} 


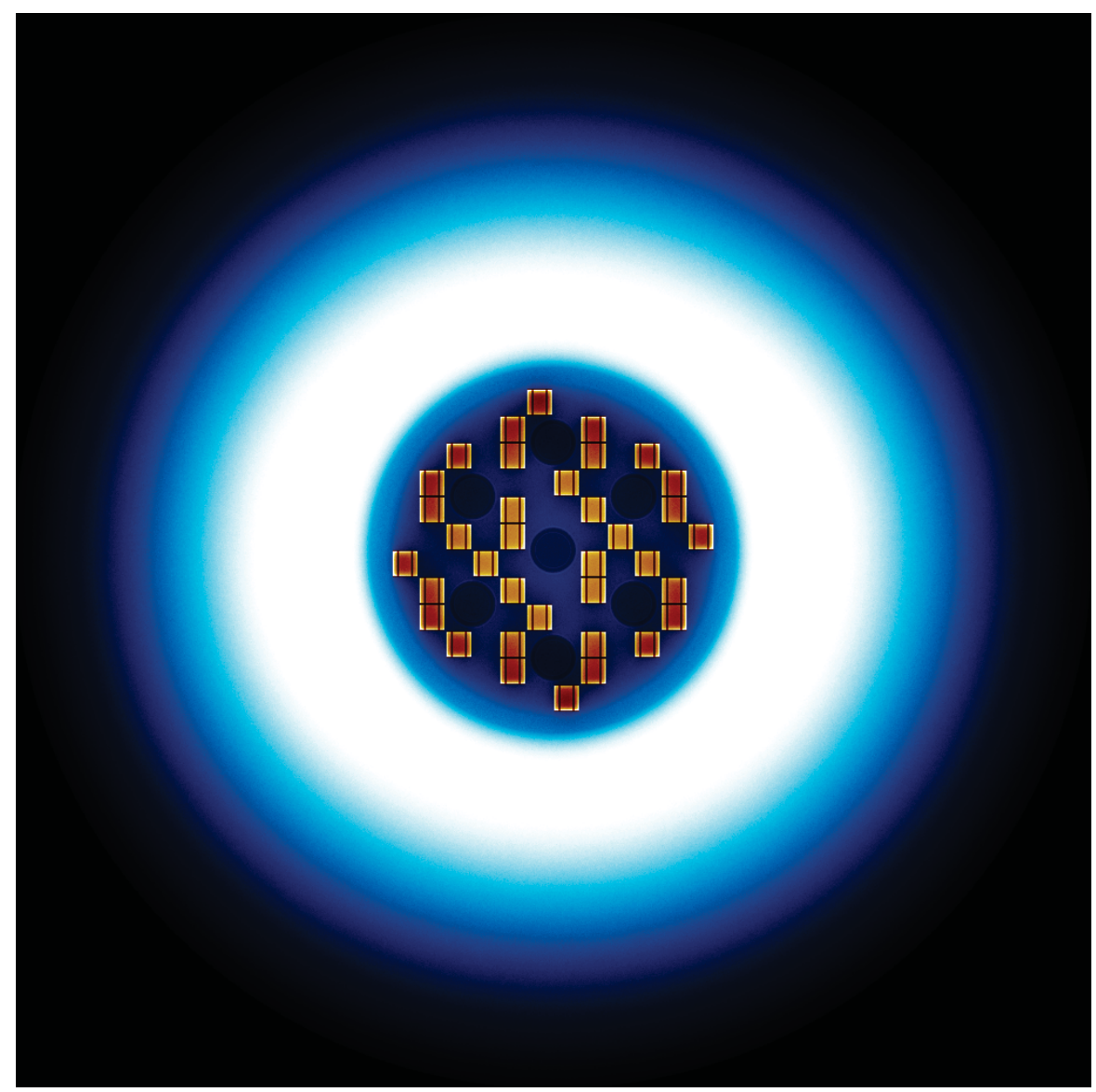

Figure 2-13. Neutron flux and power map of Square core model having all fresh fuel.

\subsection{Annular Fuel Concept}

The "Annular" fuel core concept is described in this section.

Table 2-5 presents the basic parameters of this reactor. The water-cooled fuel assemblies are arcuate (similar in basic shape to ATR assemblies) and are comprised of 12 plates of U-10Mo fuel meat clad in Zircaloy-4. Fuel plates again have an outer thickness of $0.125 \mathrm{~cm}$ and a meat 
thickness of $0.05 \mathrm{~cm}$. Each arcuate fuel assembly subtends an angle of $45^{\circ}$, as is shown in Figure 2-14. The total mass of uranium in the baseline Annular core is $493 \mathrm{~kg}, 97 \mathrm{~kg}$ of which is ${ }^{235} \mathrm{U}$. Side plates of the fuel assemblies are $0.475 \mathrm{~cm}$ wide and coolant channels are again $2 \mathrm{~mm}$ wide. Though it may be required in a final design, no offset of fuel from the side plates is assumed at this time. The fuel plate cladding and the outer can of the assemblies are Zircaloy.

Figure 2-15 shows a schematic view of the Annular core concept. The 56 arcuate fuel assemblies are arranged in groups of eight surrounding seven flux traps, each having an associated IPT. The fuel assemblies sit in an aluminum core rack in the same way as described in the Cylindrical and the Square baseline designs. The aluminum rack is again assumed to contain $5 \% \mathrm{H}_{2} \mathrm{O}$ coolant by volume for cooling. An aluminum pressure vessel having inner and outer radii of 51 and $55 \mathrm{~cm}$, respectively surrounds the core rack. Outside the pressure vessel is the atmospheric pressure $\mathrm{D}_{2} \mathrm{O}$ tank.

Figure 2-16 shows the layout of fuel assemblies surrounding one of the peripheral flux traps. The IPT is located at the center with the same piping as was described for the Cylindrical and the Square designs, only now they are reduced in diameter with the central test space having a diameter of $7 \mathrm{~cm}$. This was done in order to accommodate the anticipated need for safety (ultimate shutdown) rods between the IPT and the fuel assemblies. This arrangement is the same as that found in six of the nine main flux traps in ATR. The worth and design of the safety rods have not been evaluated, but aluminum followers and a guide tube are included in the model at this stage.

Figure 2-17 shows the fuel and IPT surrounding the central flux trap. In this case, no safety rod is included and the flux trap and IPT hardware are expanded to fill the available space. In this case, the diameter of the available test space is approximately $13 \mathrm{~cm}$. Figure 2-18 shows another above view of the Annular core concept with the $\mathrm{D}_{2} \mathrm{O}$ tank and the reflector tank flux tally positions shown. Figure 2-19 shows an axial view of a center cross-section of the core. As can be seen in the figure the assemblies again have homogenized regions extending above and below the $120 \mathrm{~cm}$ active core region. The IPTs extend the entire height of the $3 \mathrm{~m}$ tall $\mathrm{D}_{2} \mathrm{O}$ tank and a void boundary is assumed beyond this for neutronic calculations.

Results from Serpent depletion calculations indicate that the baseline Annular reactor has an initial $k_{\text {eff }}$ of 1.13172 and a $B_{1}$ value of 63 days. Table 2-6 shows neutron flux in various positions in the Annular core. The center flux trap has a peak fast and total flux of $3.0 \times 10^{14}$ $\mathrm{n} \cdot \mathrm{cm}^{-2} \cdot \mathrm{s}^{-1}$ and $2.0 \times 10^{15} \mathrm{n} \cdot \mathrm{cm}^{-2} \cdot \mathrm{s}^{-1}$, respectively, and the axial flux peaking factor in the center flux trap is 1.3. The peripheral flux traps have a peak fast and total flux of $1.9 \times 10^{14} \mathrm{n} \cdot \mathrm{cm}^{-2} \cdot \mathrm{s}^{-1}$ and $1.3 \times 10^{15} \mathrm{n} \cdot \mathrm{cm}^{-2} \cdot \mathrm{s}^{-1}$, respectively. Reflector tally position \#1 has a peak total neutron flux of $9.0 \times 10^{14} \mathrm{n} \cdot \mathrm{cm}^{-2} \cdot \mathrm{s}^{-1}$. This reactor exhibits generally lower flux values in the flux traps than those of the Cylindrical and Square cores. This is likely due to the amount of IPT and control rod hardware and its associated cooling between the fuel assemblies and the test spaces. It should be noted, however, that the control elements have not been designed in detail for any of the concepts. Therefore, differences in performance stemming from effects of control elements should be considered accordingly. Figure 2-20 shows a neutron flux map of the all-fresh Annular core at beginning of irradiation. 
Table 2-5. Parameters of baseline annular fuel assemblies and core.

\begin{tabular}{|l|c|}
\hline Parameter & \\
\hline Active Fuel Height $(\mathrm{cm})$ & 120 \\
\hline Fuel Meat Material & $\mathrm{U}-10 \mathrm{Mo}$ \\
\hline${ }^{235} \mathrm{U}$ density $\left(\mathrm{g} / \mathrm{cm}^{3}\right)$ & 3.00 \\
\hline Total U density $\left(\mathrm{g} / \mathrm{cm}^{3}\right)$ & 15.2 \\
\hline Enrichment $(\mathrm{wt} . \%)$ & 19.7 \\
\hline Number of Assemblies & 56 \\
\hline Fuel Plates Per Assembly & 12 \\
\hline Inner diameter of innermost coolant channel $(\mathrm{cm})$ & 23.0 \\
\hline Outer diameter of outermost coolant channel $(\mathrm{cm})$ & 31.2 \\
\hline Width of coolant channels between fuel plates $(\mathrm{cm})$ & 0.2 \\
\hline Fuel plate thickness $(\mathrm{cm})$ & 0.125 \\
\hline Fuel meat thickness $(\mathrm{cm})$ & 0.05 \\
\hline Cladding Composition & Zircaloy-4 \\
\hline Side Plate Composition & Zircaloy-4 \\
\hline Side Plate Width $(\mathrm{cm})$ & 0.475 \\
\hline Total ${ }^{235} \mathrm{U}$ per assembly $(\mathrm{kg})$ & 1.74 \\
\hline Total ${ }^{238} \mathrm{U}$ per assembly $(\mathrm{kg})$ & 7.06 \\
\hline Total ${ }^{235} \mathrm{U}$ in core $(\mathrm{kg})$ & 97.3 \\
\hline Total ${ }^{238} \mathrm{U}$ in core $(\mathrm{kg})$ & 395.6 \\
\hline
\end{tabular}




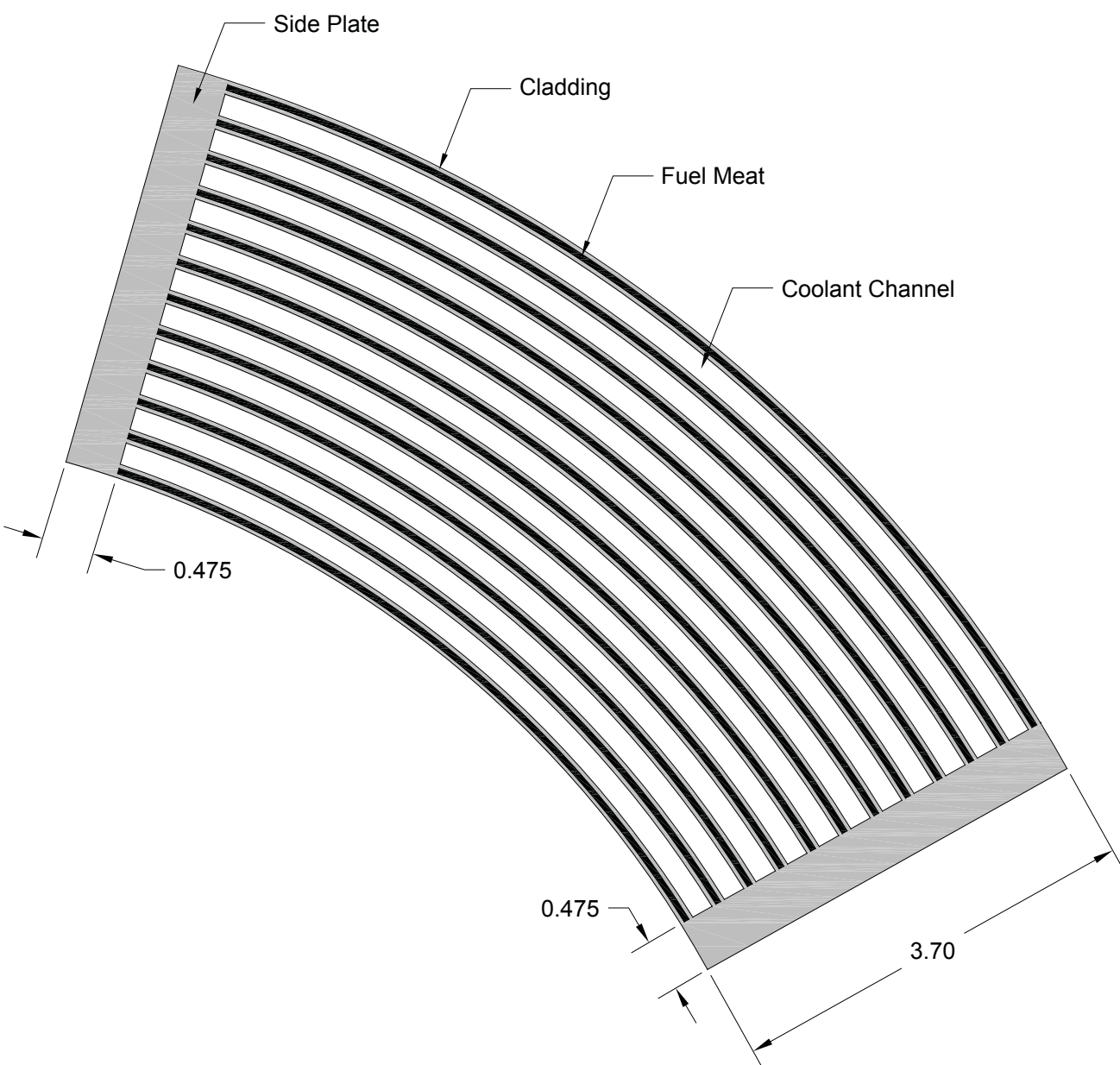

Figure 2-14. Cutaway of arcuate fuel assembly of the annular concept. 


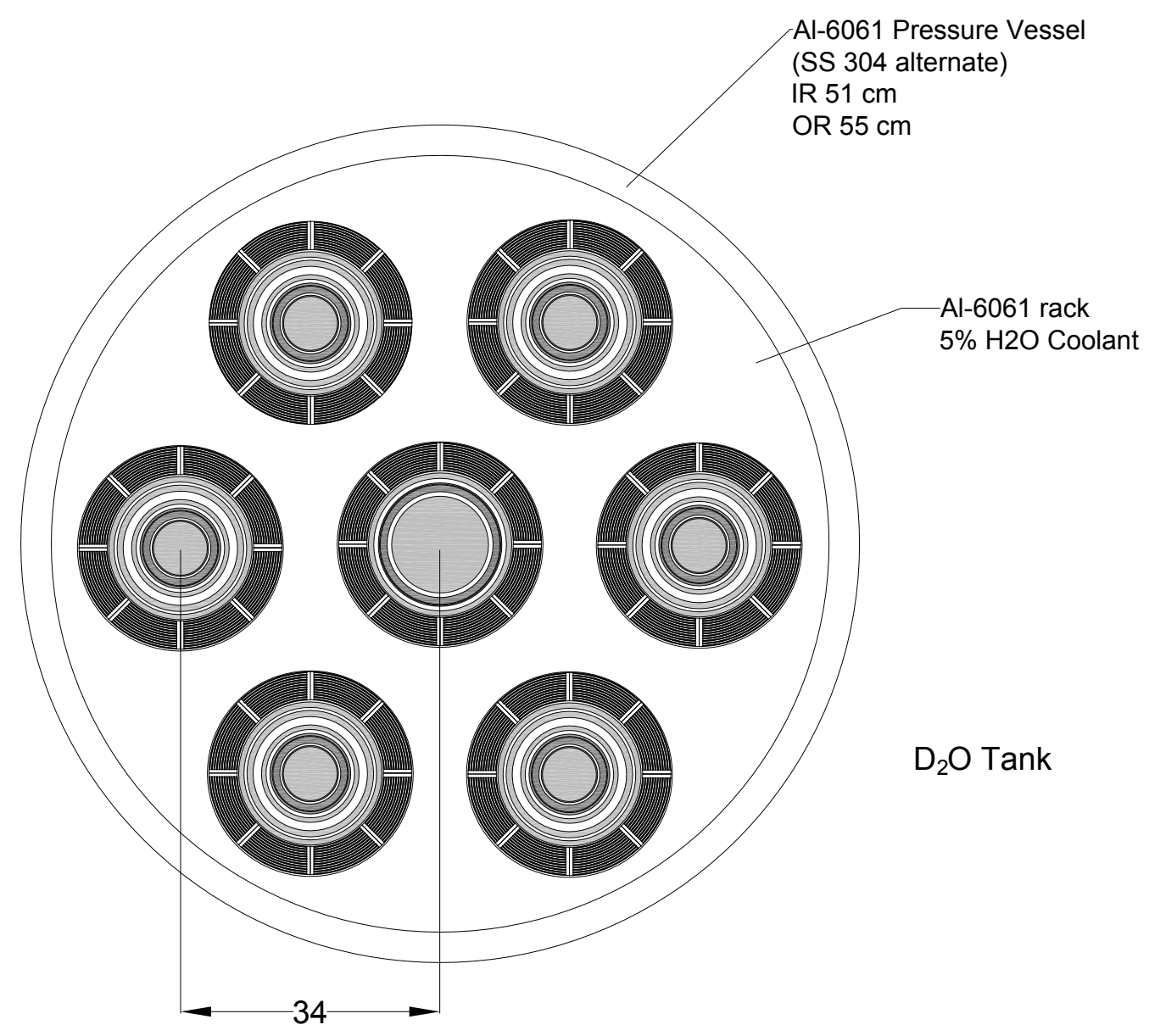

Figure 2-15. Detailed view of annular core concept (dimensions in $\mathrm{cm}$ ). 


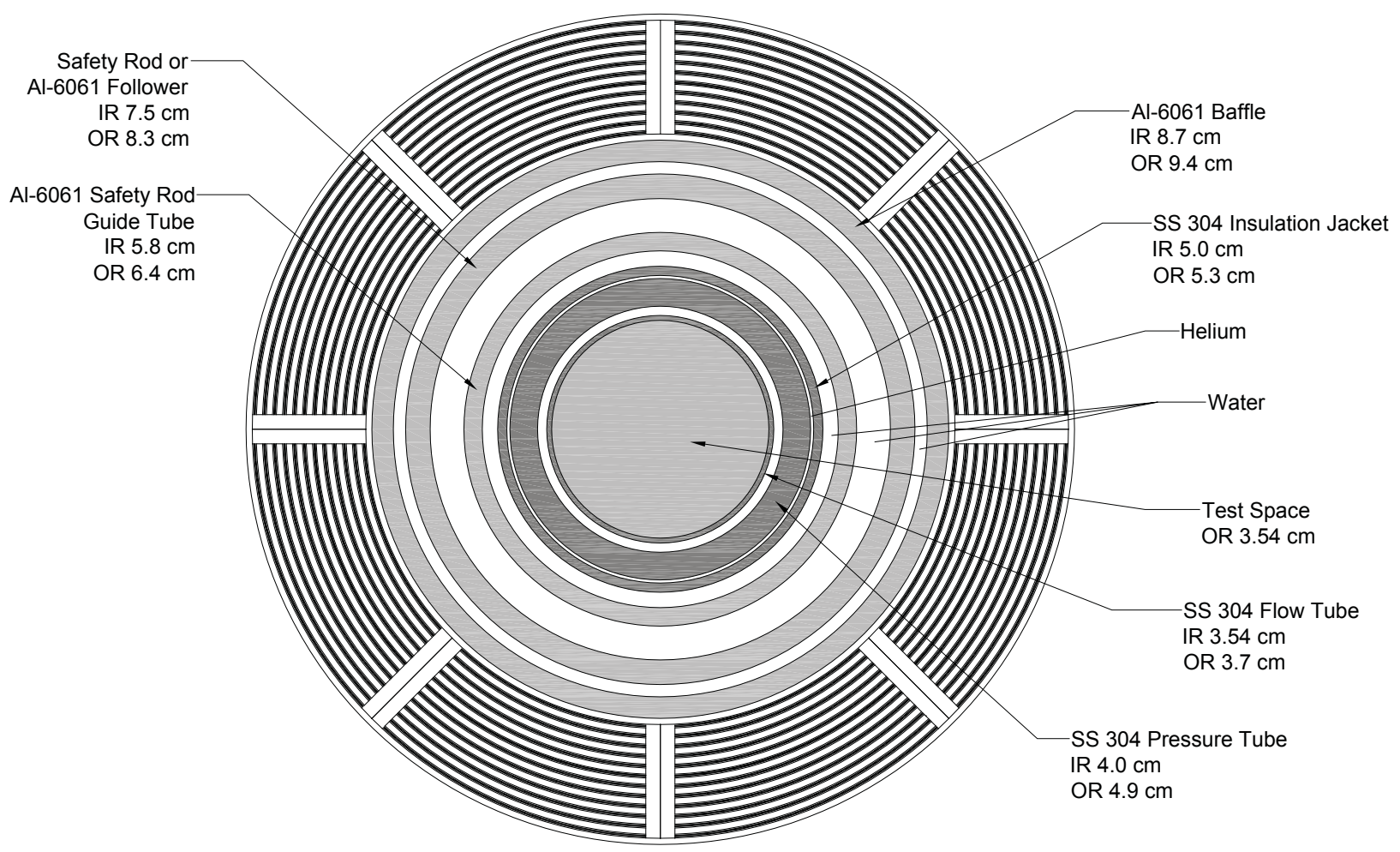

Figure 2-16. Schematic of peripheral module of annular design with safety rod. 


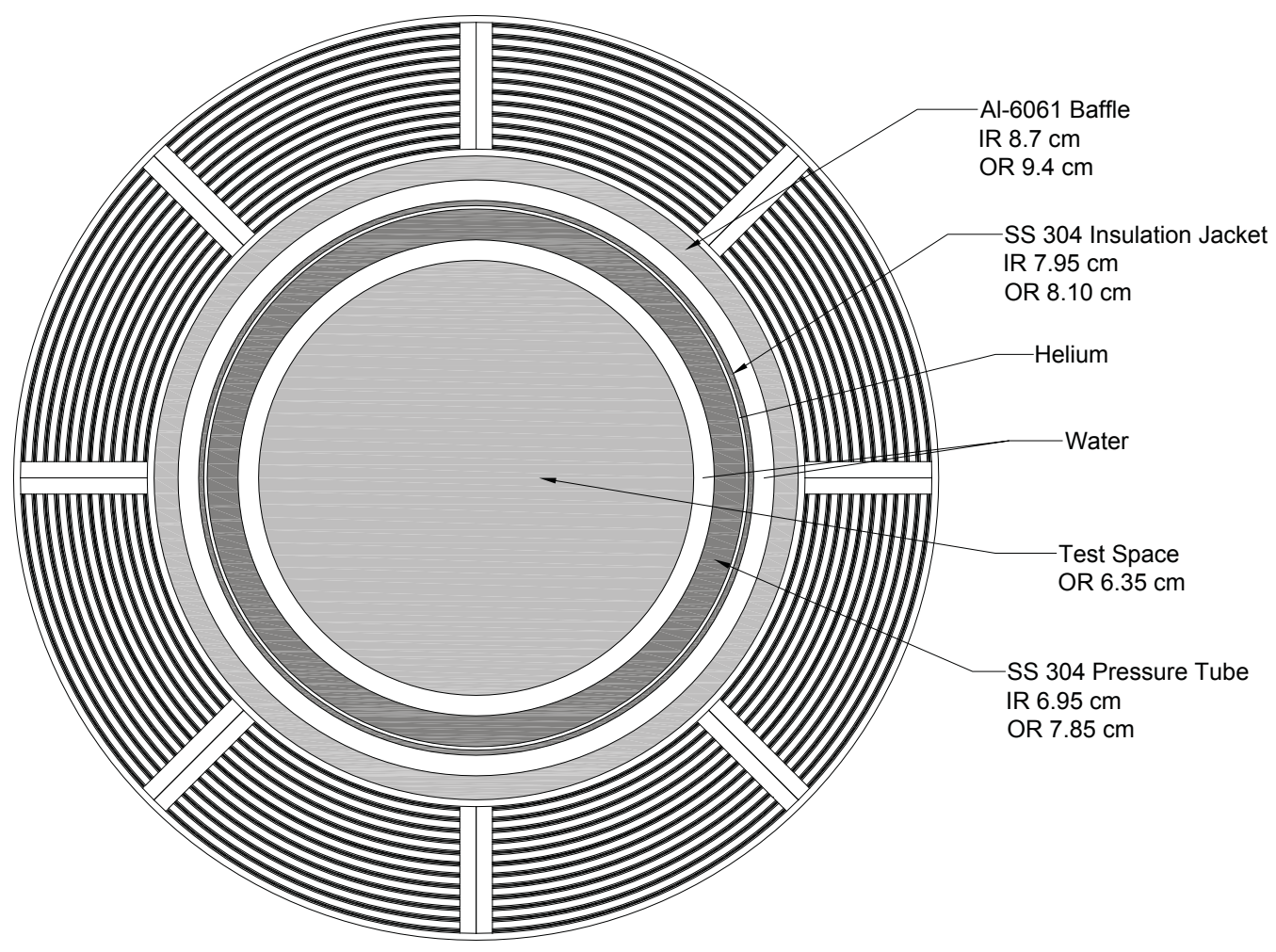

Figure 2-17. Schematic of center module of annular design with no safety rod. 


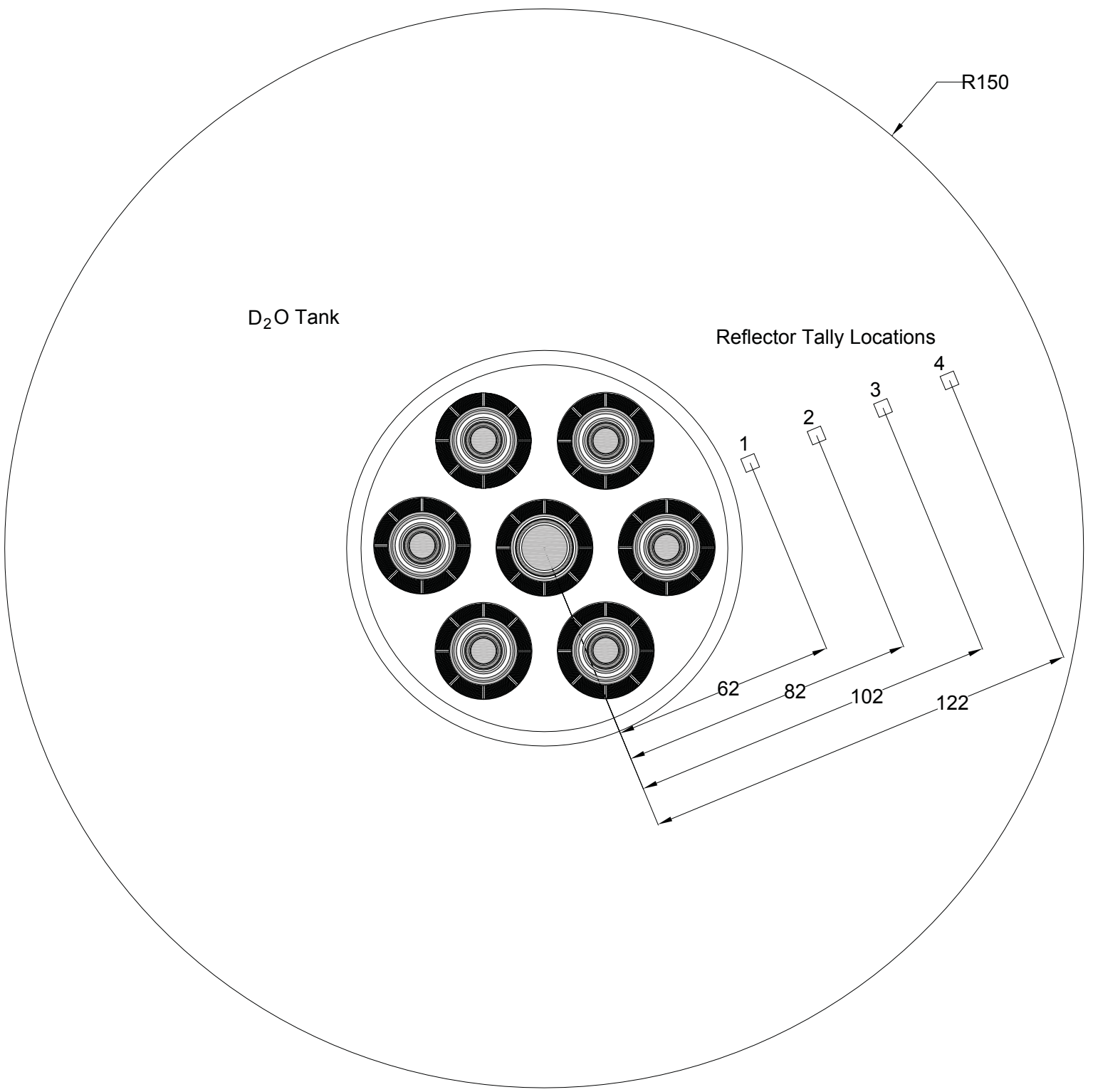

Figure 2-18. Above view of annular concept with reflector tally locations (dimensions in $\mathrm{cm}$ ). 

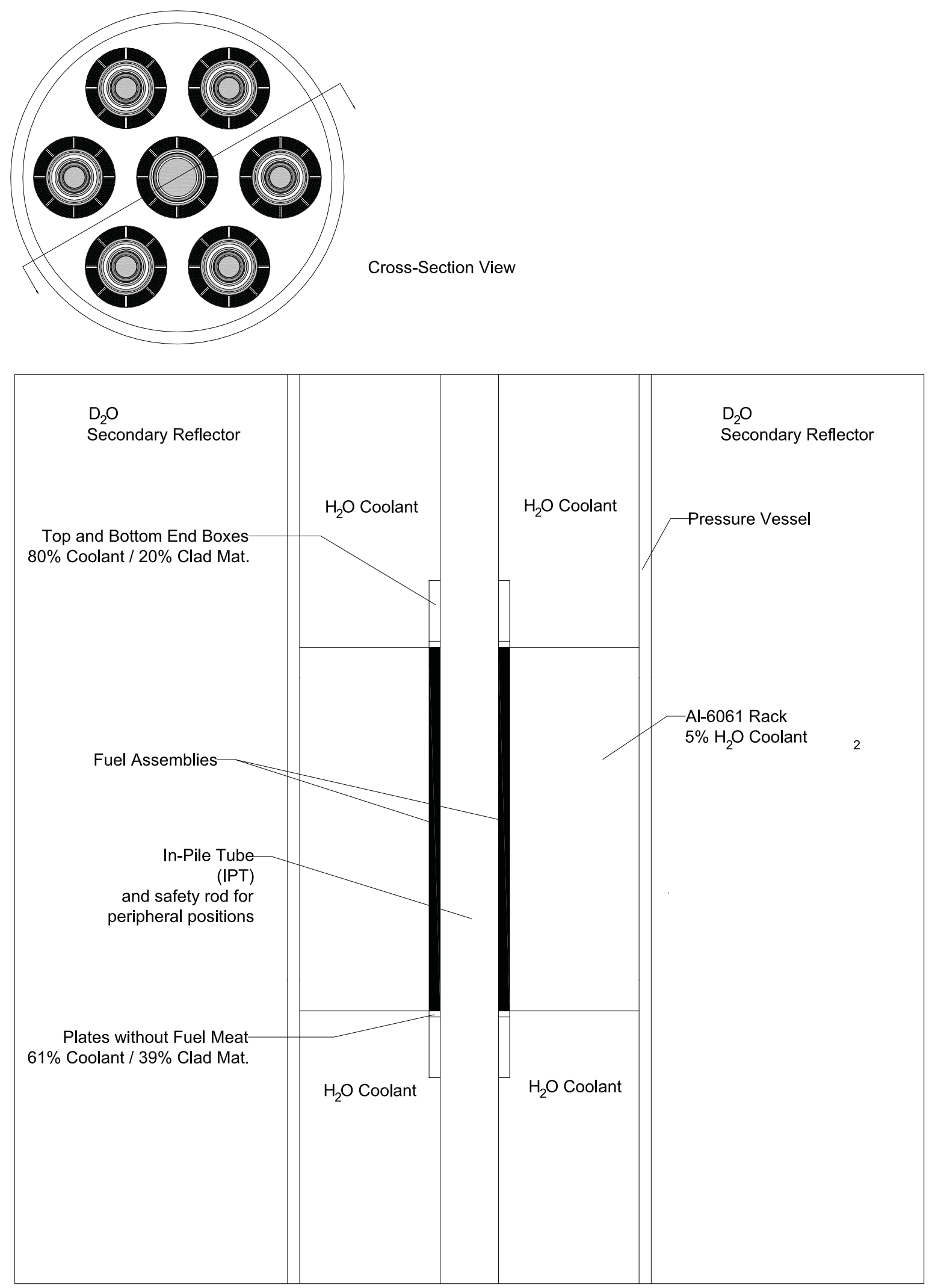

Figure 2-19. Vertical cross section view of annular core concept. 
Table 2-6. Neutron flux in various locations in the Annular core. ${ }^{\text {a }}$

\begin{tabular}{|c|c|c|c|}
\hline \multirow[t]{2}{*}{ Tally Location } & \multicolumn{3}{|c|}{$\begin{array}{c}\text { Neutron Flux in Reflector }\left(\mathrm{n} \cdot \mathrm{cm}^{-2} \cdot \mathrm{s}^{-1}\right) \\
\text { Fast Flux }(>1 \mathrm{MeV}) \\
\text { Thermal Flux }(<0.625 \mathrm{eV}) \\
\text { Total Flux }\end{array}$} \\
\hline & Peak & $\begin{array}{c}\text { Axially } \\
\text { Averaged }\end{array}$ & $\begin{array}{c}\text { Peak/ } \\
\text { Average }\end{array}$ \\
\hline \multirow{3}{*}{ Center Flux Trap } & $2.99 \mathrm{E}+14$ & $2.28 \mathrm{E}+14$ & 1.31 \\
\hline & $3.57 \mathrm{E}+14$ & $2.75 \mathrm{E}+14$ & 1.30 \\
\hline & $2.05 \mathrm{E}+15$ & $1.56 \mathrm{E}+15$ & 1.31 \\
\hline \multirow{3}{*}{ Peripheral Flux Trap } & $1.87 \mathrm{E}+14$ & $1.43 \mathrm{E}+14$ & 1.31 \\
\hline & $2.52 \mathrm{E}+14$ & $1.93 \mathrm{E}+14$ & 1.31 \\
\hline & $1.26 \mathrm{E}+15$ & $9.57 \mathrm{E}+14$ & 1.32 \\
\hline \multirow{3}{*}{ Reflector Tally 1} & $9.92 \mathrm{E}+12$ & $7.47 \mathrm{E}+12$ & 1.33 \\
\hline & $6.45 \mathrm{E}+14$ & $5.22 \mathrm{E}+14$ & 1.24 \\
\hline & $8.96 \mathrm{E}+14$ & $7.12 \mathrm{E}+14$ & 1.26 \\
\hline \multirow{3}{*}{ Reflector Tally 2} & - & - & - \\
\hline & $6.00 \mathrm{E}+14$ & $4.90 \mathrm{E}+14$ & 1.22 \\
\hline & $6.17 \mathrm{E}+14$ & $5.03 \mathrm{E}+14$ & 1.23 \\
\hline \multirow{3}{*}{ Reflector Tally 3} & - & - & - \\
\hline & $3.57 \mathrm{E}+14$ & $3.01 \mathrm{E}+14$ & 1.19 \\
\hline & $3.58 \mathrm{E}+14$ & $3.02 \mathrm{E}+14$ & 1.19 \\
\hline \multirow{3}{*}{ Reflector Tally 4} & - & - & - \\
\hline & $1.79 \mathrm{E}+14$ & $1.55 \mathrm{E}+14$ & 1.16 \\
\hline & $1.80 \mathrm{E}+14$ & $1.55 \mathrm{E}+14$ & 1.16 \\
\hline
\end{tabular}

\footnotetext{
${ }^{\mathrm{a}}$ Initial $k_{\text {eff }}=1.13172, \mathrm{~B}_{1}=63$ days
} 


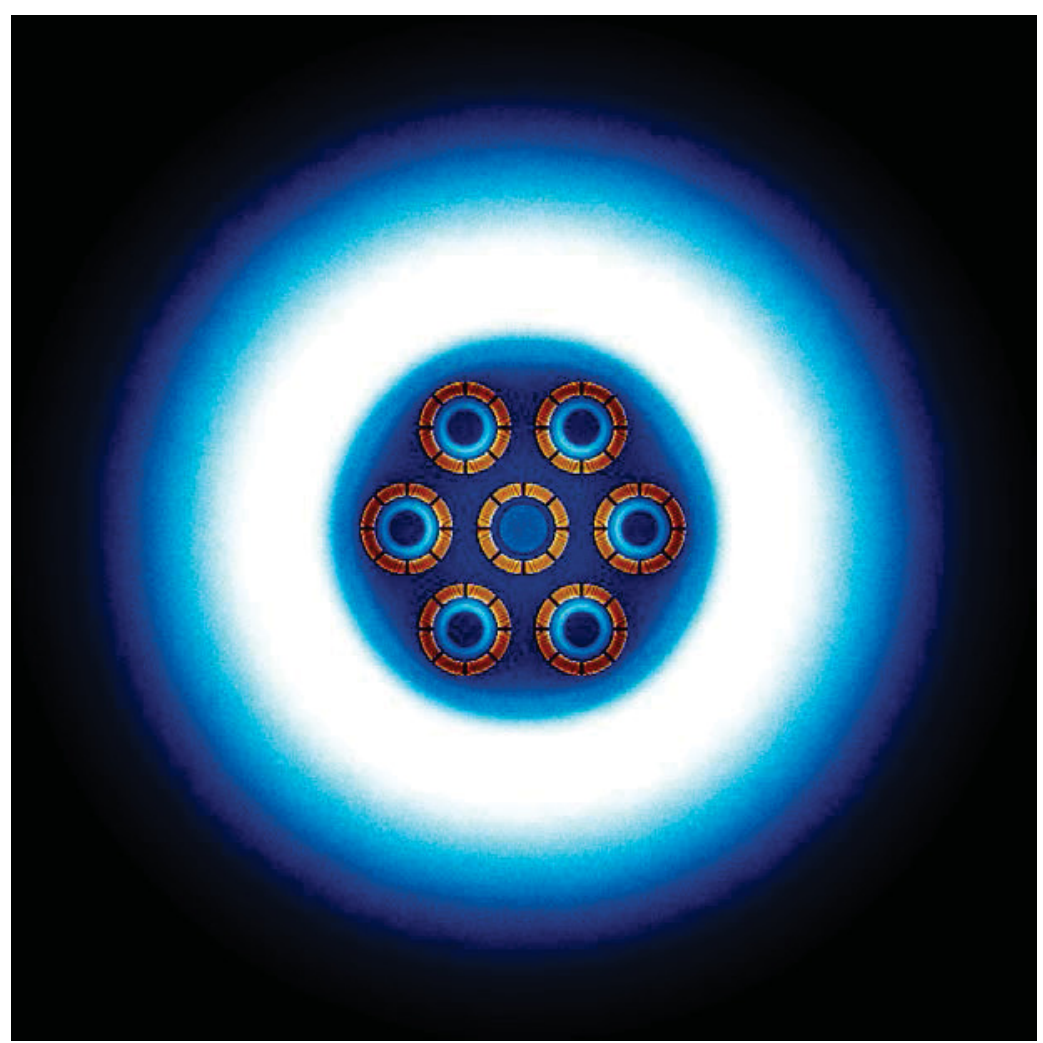

Figure 2-20. Neutron flux and power map of Annular core model having all fresh fuel.

\subsection{Pressure Boundary Tube (PBT) Concept}

The Pressure Boundary Tube (PBT) concept is very closely related to the Multiple-Annular (M-A) design from the original BATR work. Table 2-7 gives the main parameters of the baseline PBT concept. The baseline configuration of the PBT concept uses the same fuel assemblies as the Annular concept (Figure 2-14), again surrounding the safety rods and IPTs. The departure from the Annular concept comes just outside the fuel elements' outer radius. Rather than the assemblies sitting in a core rack, they are positioned inside seven individual 1.1 cm-thick aluminum pressure boundary tubes (PBTs). These tubes extend the entire axial height of the $\mathrm{D}_{2} \mathrm{O}$ tank and collectively serve as the pressure boundary containing the primary $\mathrm{H}_{2} \mathrm{O}$ coolant. This is illustrated in Figure 2-21 for the center fuel grouping and in Figure 2-22 for the peripheral modules.

Figure 2-23 shows an above view of the PBT concept with the tally locations shown in the $\mathrm{D}_{2} \mathrm{O}$ reflector tank. Figure 2-24 shows an axial view of the PBT concept. As can be seen in the figure the assemblies again have homogenized regions extending above and below the $120 \mathrm{~cm}$ active core region. The IPTs extend the entire height of the $3 \mathrm{~m}$ tall $\mathrm{D}_{2} \mathrm{O}$ tank and a void boundary is assumed beyond this for neutronic calculations. 
Results from Serpent 2 depletion calculations indicate that the baseline PBT reactor has an initial $k_{\text {eff }}$ of 1.32352 and a $B_{1}$ value of 184 days. Table 2-8 shows neutron flux in various positions in the PBT core. The center flux trap has a peak fast and total flux of $2.2 \times 10^{14} \mathrm{n}^{\cdot} \mathrm{cm}^{-2} \cdot \mathrm{s}^{-}$ ${ }^{1}$ and $1.5 \times 10^{15} \mathrm{n} \cdot \mathrm{cm}^{-2} \cdot \mathrm{s}^{-1}$, respectively and the axial flux peaking factor in the center flux trap is again 1.3. The peripheral flux traps have a peak fast and total flux of $1.7 \times 10^{14} \mathrm{n} \cdot \mathrm{cm}^{-2} \cdot \mathrm{s}^{-1}$ and $1.1 \times 10^{15} \mathrm{n} \cdot \mathrm{cm}^{-2} \cdot \mathrm{s}^{-1}$, respectively. Reflector tally position \#1 has a peak total neutron flux of $1.3 \times 10^{15} \mathrm{n} \cdot \mathrm{cm}^{-2} \cdot \mathrm{s}^{-1}$. This reactor exhibits much higher reactivity than the other cores. This is due to the presence of the very effectively moderating $\mathrm{D}_{2} \mathrm{O}$ in close proximity to fuel. This is in contrast to the low-moderating aluminum core rack used in the other concepts. It should be again noted that the control elements assumed here have not been designed in detail. Figure 2-25 shows a neutron flux map of the all-fresh PBT core at beginning of irradiation. This figure illustrates the high thermal flux close to the outer boundary of the fuel compared to the relatively hard spectrum nominally present in the reactors having aluminum core racks.

Table 2-7. Parameters of baseline PBT concept fuel assemblies and core.

\begin{tabular}{|l|c|}
\hline Parameter & \\
\hline Active Fuel Height $(\mathrm{cm})$ & 100 \\
\hline Fuel Meat Material & $\mathrm{U}-10 \mathrm{Mo}$ \\
\hline${ }^{235} \mathrm{U}$ density $\left(\mathrm{g} / \mathrm{cm}^{3}\right)$ & 3.00 \\
\hline Total U density $\left(\mathrm{g} / \mathrm{cm}^{3}\right)$ & 15.2 \\
\hline Enrichment $(\mathrm{wt} . \%)$ & 19.7 \\
\hline Number of Assemblies & 56 \\
\hline Fuel Plates Per Assembly & 12 \\
\hline Inner diameter of innermost coolant channel $(\mathrm{cm})$ & 23.0 \\
\hline Outer diameter of outermost coolant channel $(\mathrm{cm})$ & 31.2 \\
\hline Width of coolant channels between fuel plates $(\mathrm{cm})$ & 0.2 \\
\hline Fuel plate thickness $(\mathrm{cm})$ & 0.125 \\
\hline Fuel meat thickness $(\mathrm{cm})$ & 0.05 \\
\hline Cladding Composition & Zircaloy-4 \\
\hline Side Plate Composition & Zircaloy-4 \\
\hline Side Plate Thickness $(\mathrm{cm})$ & 0.475 \\
\hline Total ${ }^{235} \mathrm{U}$ per assembly $(\mathrm{kg})$ & 1.74 \\
\hline Total ${ }^{238} \mathrm{U}$ per assembly $(\mathrm{kg})$ & 7.06 \\
\hline Total ${ }^{235} \mathrm{U}$ in core $(\mathrm{kg})$ & 97.3 \\
\hline Total ${ }^{238} \mathrm{U}$ in core $(\mathrm{kg})$ & 395.6 \\
\hline
\end{tabular}




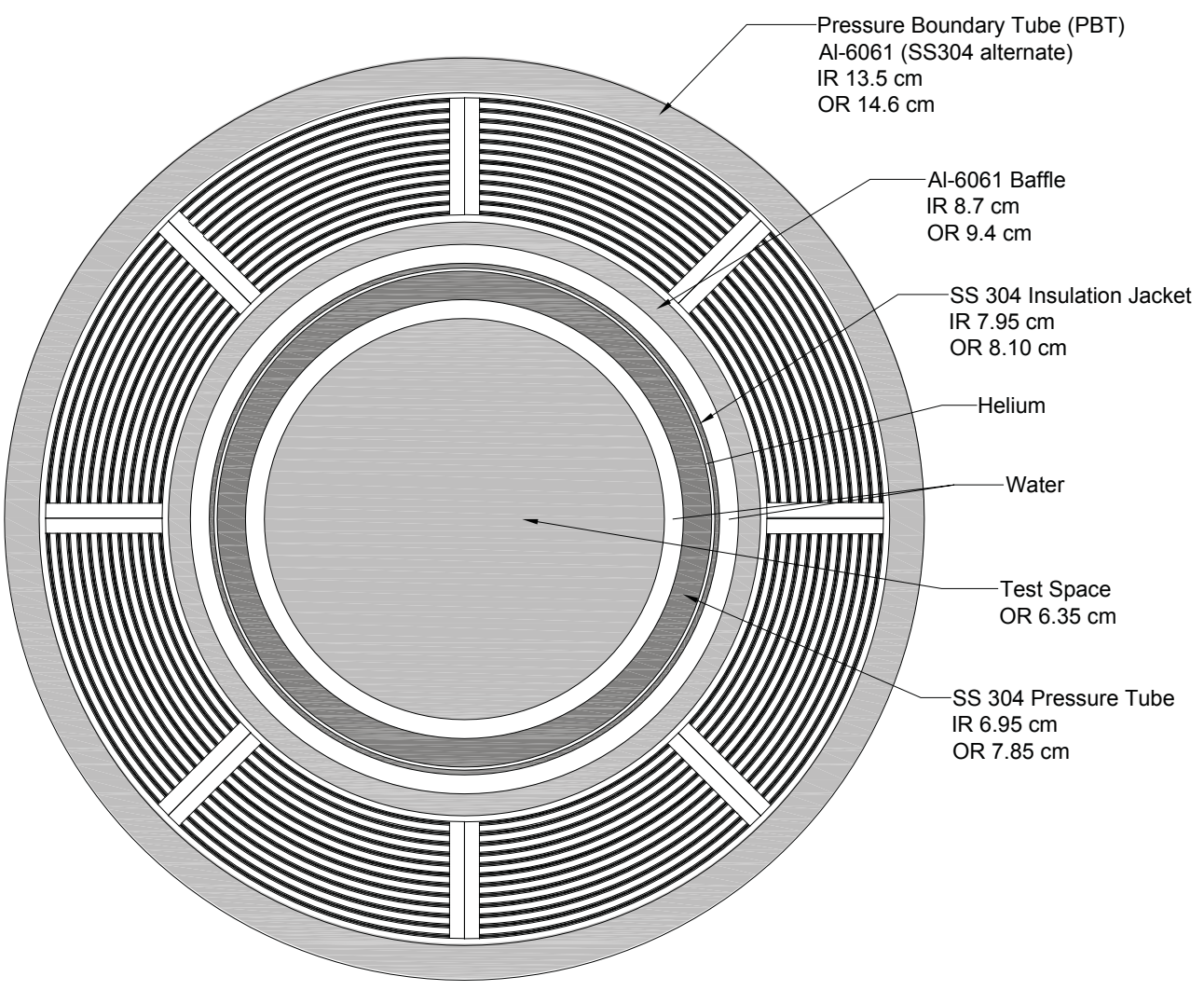

Figure 2-21. Schematic of center module of PBT design with no safety rod. 


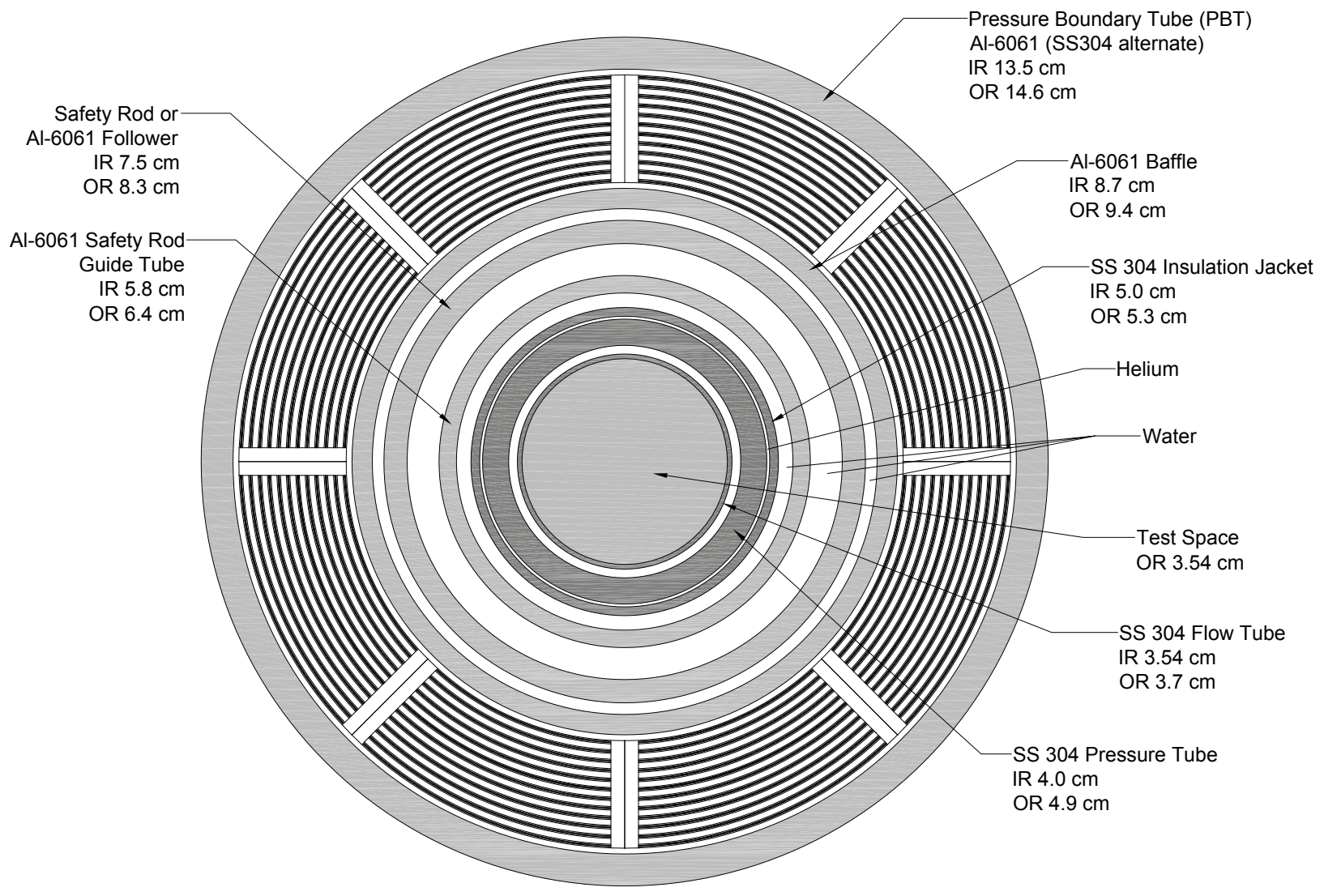

Figure 2-22. Schematic of peripheral module of PBT design with no safety rod. 


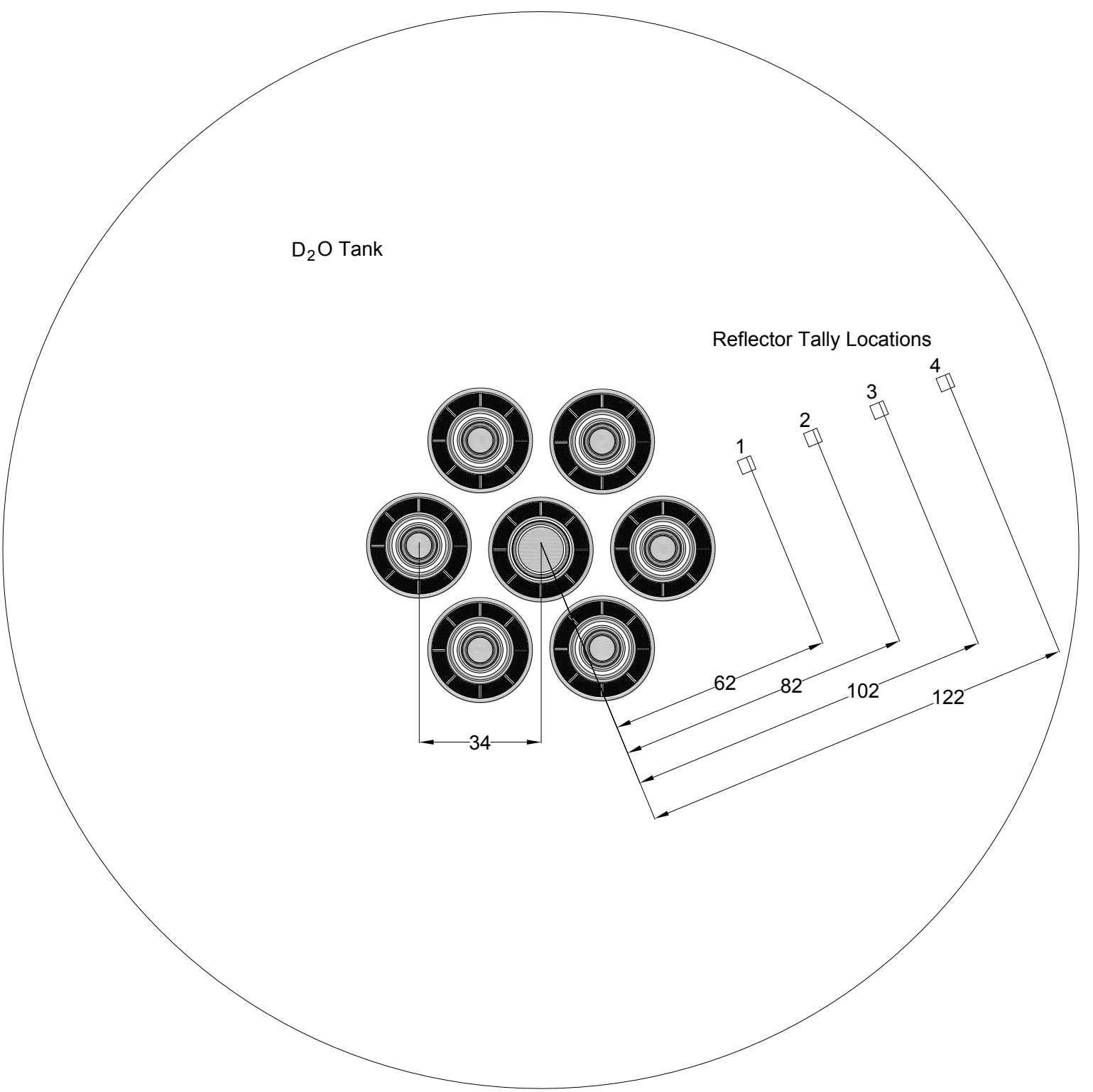

Figure 2-23. Above view of PBT concept with reflector tally locations (dimensions in $\mathrm{cm}$ ). 
Cross-Section View
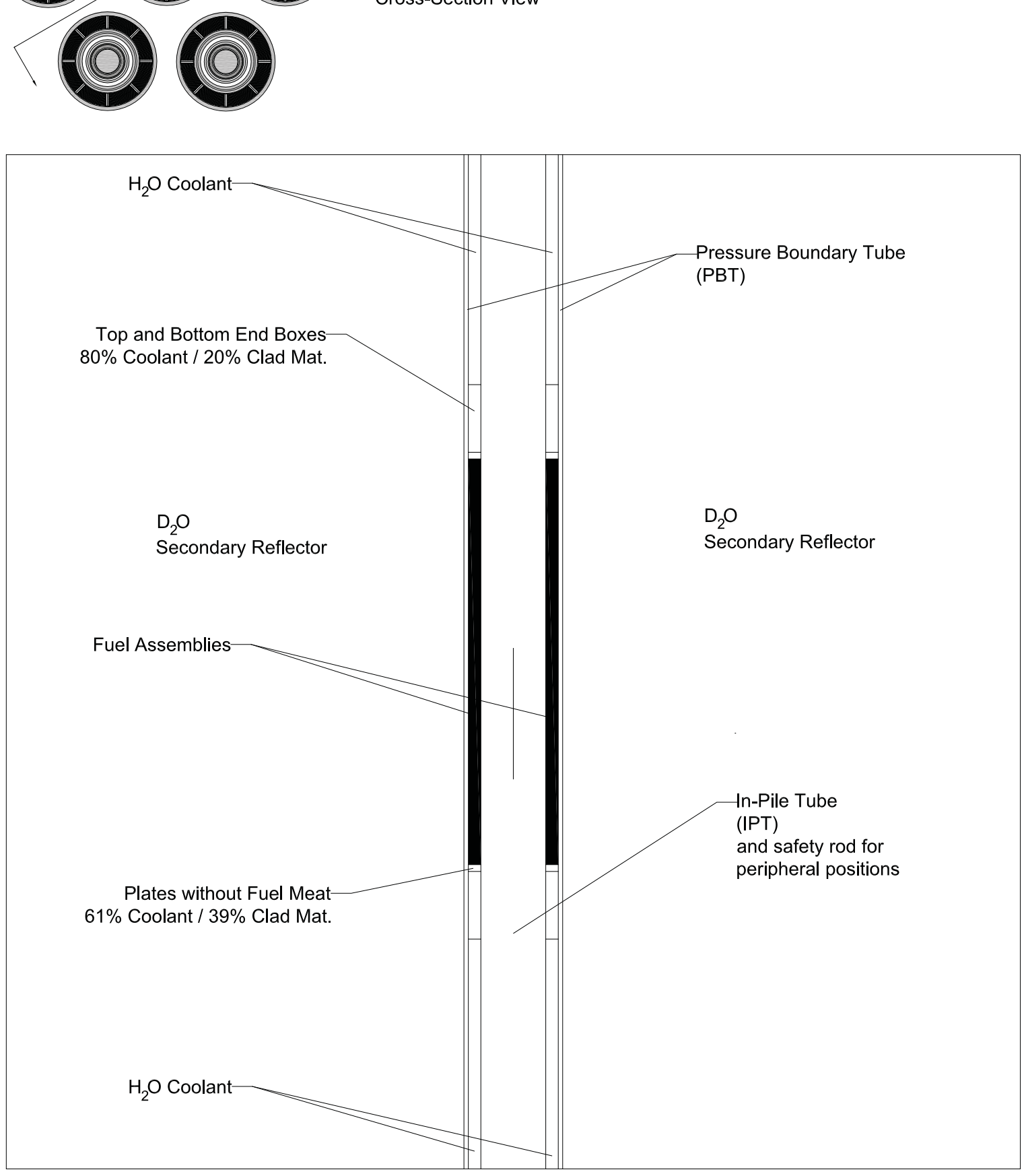

Figure 2-24. Vertical cross section view of PBT concept. 
Table 2-8. Neutron flux in various locations in the PBT core. ${ }^{\text {a }}$

\begin{tabular}{|c|c|c|c|}
\hline \multirow[t]{2}{*}{ Tally Location } & \multicolumn{3}{|c|}{$\begin{array}{c}\text { Neutron Flux in Reflector }\left(\mathrm{n} \cdot \mathrm{cm}^{-2} \cdot \mathrm{s}^{-1}\right) \\
\text { Fast Flux }(>1 \mathrm{MeV}) \\
\text { Thermal Flux }(<0.625 \mathrm{eV}) \\
\text { Total Flux }\end{array}$} \\
\hline & Peak & $\begin{array}{l}\text { Axially } \\
\text { Averaged }\end{array}$ & $\begin{array}{c}\text { Peak/ } \\
\text { Average }\end{array}$ \\
\hline \multirow{3}{*}{ Center Flux Trap } & $2.23 \mathrm{E}+14$ & $1.70 \mathrm{E}+14$ & 1.32 \\
\hline & $2.98 \mathrm{E}+14$ & $2.29 \mathrm{E}+14$ & 1.30 \\
\hline & $1.53 \mathrm{E}+15$ & $1.17 \mathrm{E}+15$ & 1.31 \\
\hline \multirow{3}{*}{ Peripheral Flux Trap } & $1.68 \mathrm{E}+14$ & $1.28 \mathrm{E}+14$ & 1.32 \\
\hline & $2.34 \mathrm{E}+14$ & $1.82 \mathrm{E}+14$ & 1.28 \\
\hline & $1.10 \mathrm{E}+15$ & $8.44 \mathrm{E}+14$ & 1.30 \\
\hline \multirow{3}{*}{ Reflector Tally 1} & $4.75 \mathrm{E}+12$ & $3.70 \mathrm{E}+12$ & 1.28 \\
\hline & $1.14 \mathrm{E}+15$ & $9.38 \mathrm{E}+14$ & 1.22 \\
\hline & $1.26 \mathrm{E}+15$ & $1.03 \mathrm{E}+15$ & 1.23 \\
\hline \multirow{3}{*}{ Reflector Tally 2} & - & - & - \\
\hline & $7.84 \mathrm{E}+14$ & $6.60 \mathrm{E}+14$ & 1.19 \\
\hline & $7.91 \mathrm{E}+14$ & $6.66 \mathrm{E}+14$ & 1.19 \\
\hline \multirow{3}{*}{ Reflector Tally 3} & - & - & - \\
\hline & $4.62 \mathrm{E}+14$ & $3.96 \mathrm{E}+14$ & 1.17 \\
\hline & $4.63 \mathrm{E}+14$ & $3.96 \mathrm{E}+14$ & 1.17 \\
\hline \multirow{3}{*}{ Reflector Tally 4} & - & - & - \\
\hline & $2.33 \mathrm{E}+14$ & $2.04 \mathrm{E}+14$ & 1.14 \\
\hline & $2.33 \mathrm{E}+14$ & $2.04 \mathrm{E}+14$ & 1.14 \\
\hline
\end{tabular}

${ }^{\mathrm{a}}$ Initial $k_{\text {eff }}=1.32352, B_{1}=184$ days 


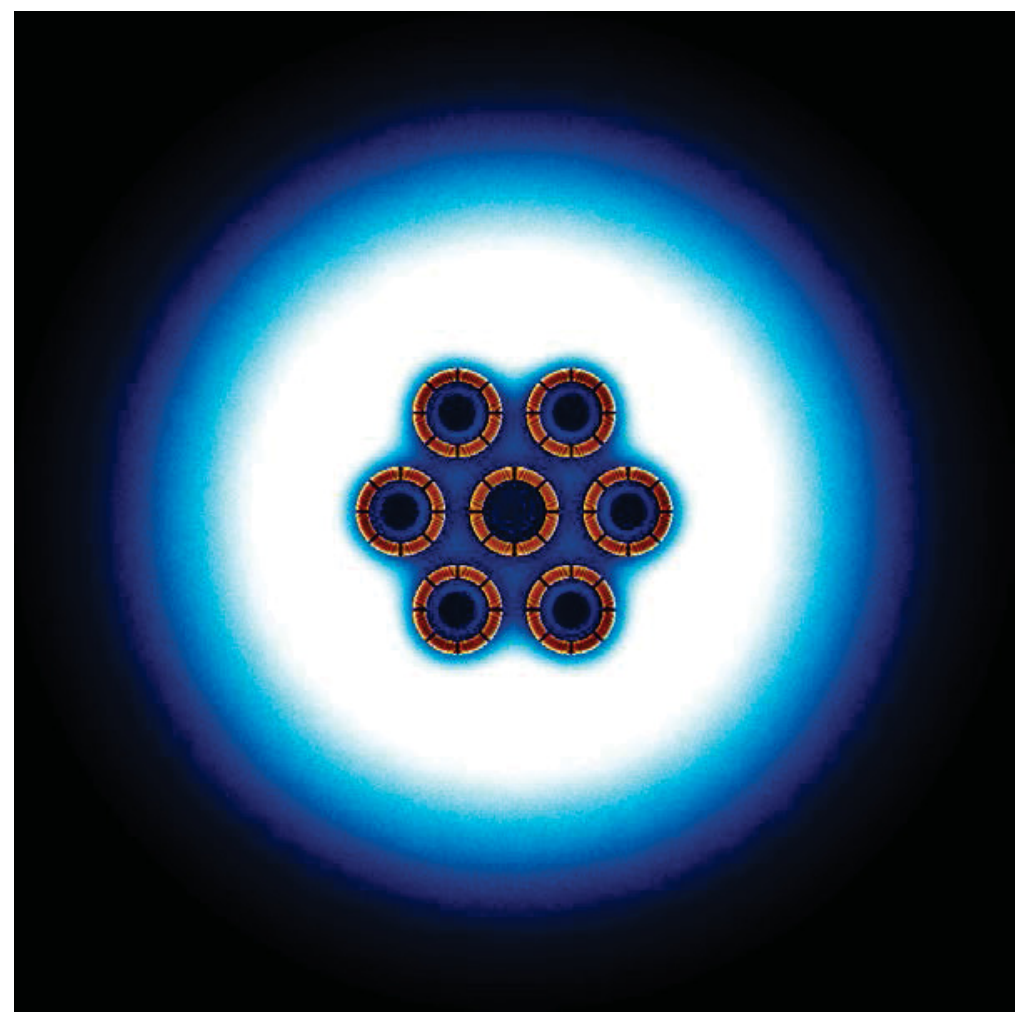

Figure 2-25. Neutron flux map of PBT core model loaded with all fresh fuel.

\section{Pressure Vessel and PBT Considerations}

In reactor designs featuring a reactor pressure vessel (RPV) located close to the fueled portion of the core, vessel materials with low neutron capture cross-section are favorable for two main reasons. One reason is that the vessel is close enough to fuel that neutron capture affects core reactivity substantially, particularly in the cases where a low-moderation core rack is used. The other motivation for a low-absorption RPV is the desire for high flux outside the RPV. Aluminum (specifically Al-6061) was identified as a candidate material to serve as a lowabsorption pressure boundary in the BATR project [3]. It is also the material to be used in the RPV for the Jules Horowitz Reactor at CEA, Cadarache[13]. An alternative fallback material is the more conventional stainless steel 304 (SS304). In this section, the existing ASME Boiler and Pressure Vessel Code [Ref ASME code] is used to determine minimum RPV thickness required for three materials; SS304, Al-6061 T4, and Al-6061 T6. The T4 and T6 designations represent different heat treatments on the same starting material. Table 3-1 gives maximum allowable stress for the three materials for the various temperatures were the code exists. For Al-6061, this extends only to $400^{\circ} \mathrm{F}\left(204^{\circ} \mathrm{C}\right)$. A minimum wall thickness $(t)$ for a pressure vessel can then be determined using the following equation [Ref ASME code]:

$$
\mathrm{t}=P^{*} R /\left(S_{m}-0.5 P\right)
$$


where $P$ is design pressure, $R$ is inner radius of the vessel, and $S_{m}$ is maximum allowable stress as given in the code. Values for a $1 \mathrm{~m}$ diameter vessel and $2.5 \mathrm{MPa}$ design pressure are given in the rightmost column in Table 1. These are plotted in Figure 3-1 as well.

As can be seen in the Table 3-1 and Figure 3-1, the allowable stress for the Al6061 T6 is higher than that of the $\mathrm{T} 4$ up to $177^{\circ} \mathrm{C}\left(350^{\circ} \mathrm{F}\right)$, where the $\mathrm{T} 6$ assumes the $\mathrm{T} 4$ value. Beyond this temperature up to $204^{\circ} \mathrm{C}\left(400^{\circ} \mathrm{F}\right)$, both of these essentially lose their heat treatment and the allowable stress drops to the $\mathrm{T} 0$ (or untreated) value. On the other hand, the maximum allowable stress for the SS304 is higher than either type of aluminum and only changes slightly in the temperature range of interest. If we assume a design pressure of $2.5 \mathrm{MPa}$, a temperature of up to $204^{\circ} \mathrm{C}$, and a vessel diameter of $1 \mathrm{~m}$, the minimum wall thickness for either aluminum type would be $3.6 \mathrm{~cm}$. Under the same conditions, the minimum wall thickness for SS304 would be 1 $\mathrm{cm}$. If the temperature of the vessel could be limited to less than $177^{\circ} \mathrm{C}$, the minimum wall thickness for Al-6061 would drop to $1.7 \mathrm{~cm}$ while that for SS304 would be essentially unchanged.

During normal operation, the RPV would not be expected to reach temperatures in excess of the $89^{\circ} \mathrm{C}$ coolant outlet temperature. The peak RPV temperature would likely occur during the period of natural convection following a primary pump coastdown.

Figure 3-2 shows the same information as Figure 3-1 except directed at the PBT option. Therefore, it shows the minimum PBT pressure boundary thickness versus temperature for $\sim 28$ cm diameter vessels.

Figure 3-3 and Figure 3-4 show radiative capture and elastic scattering cross-sections, respectively, for Fe-56 and Al-27. These are the two major constituents of the aluminum and SS304 material candidates examined here in detail.

Table 3-2 shows results of neutronic calculations with the candidate pressure vessel materials for each of the baseline MATRIX concepts. From these results, one sees that in all cases, use of a SS304 pressure boundary carries a heavy reactivity penalty over aluminum. This is due primarily to the much larger absorption cross-section seen in Figure 3-3. This is particularly true in the PBT case since the pressure boundary is closest to the fuel in this concept. However, the PBT core has enough excess reactivity to absorb the loss in reactivity and remain viable. One also may note that although the SS304 material carries a significant reactivity penalty, flux in the flux traps and in the reflector are not affected adversely. As for the flux in the reflector, the thinness of the SS304 is likely responsible for the lack of a significant reduction in flux. Zircaloy was also added to the Cylindrical core with the same thickness as the SS304. One sees that this material performs best of all three, though costs may be prohibitive.

Note that this analysis has not considered the effects of neutron fluence and whether this would add additional thickness requirements to the RPV and how it would dictate its change-out interval.

Also note that while calculations are not included in this section for Zircaloy pressure boundaries for the PBT core, it would be expected to perform quite well neutronically (similar to aluminum) while requiring the same or smaller thickness as SS304. Future work on PBT designs should consider Zircaloy as a lead pressure boundary candidate. 
Table 3-1. Maximum allowable stress and minimum wall thickness of candidate pressure boundary materials for various maximum temperatures.

\begin{tabular}{|c|c|c|c|c|c|c|}
\hline \multirow[t]{2}{*}{ Material and Treatment } & \multicolumn{2}{|c|}{ Temperature } & \multicolumn{2}{|c|}{$\begin{array}{c}\text { Maximum Allowable Stress } \\
\text { [Ref. ASME Code] }\end{array}$} & \multicolumn{2}{|c|}{$\begin{array}{l}\text { Min. Wall Thickness } \\
(\mathrm{cm})\end{array}$} \\
\hline & $\left({ }^{\circ} \mathrm{F}\right)$ & $\left({ }^{\circ} \mathrm{C}\right)$ & (ksi) & $(\mathrm{MPa})$ & $1 \mathrm{~m}$ Dia. & $28 \mathrm{~cm}$ Dia. \\
\hline \multirow{5}{*}{$\begin{array}{c}\text { Al-6061 T4 } \\
\text { Drawn, Seamless Tube }\end{array}$} & 200 & 93 & 10.4 & 71 & 1.8 & 0.50 \\
\hline & 250 & 121 & 10.2 & 69 & 1.8 & 0.51 \\
\hline & 300 & 149 & 10.2 & 69 & 1.8 & 0.51 \\
\hline & 350 & 177 & 10.2 & 69 & 1.8 & 0.51 \\
\hline & 400 & 204 & 5.2 & 35 & 3.7 & 1.0 \\
\hline \multirow{5}{*}{$\begin{array}{c}\text { Al-6061 T6 } \\
\text { Drawn, Seamless Tube }\end{array}$} & 200 & 93 & 17.5 & 119 & 1.1 & 0.30 \\
\hline & 250 & 121 & 17.5 & 119 & 1.1 & 0.30 \\
\hline & 300 & 149 & 16.7 & 114 & 1.1 & 0.31 \\
\hline & 350 & 177 & 10.7 & 73 & 1.7 & 0.49 \\
\hline & 400 & 204 & 5.2 & 35 & 3.7 & 1.0 \\
\hline \multirow{13}{*}{$\begin{array}{c}18 \mathrm{Cr}-8 \mathrm{Ni} \\
\text { Stainless Steel } 304 \\
\text { Seamless Tube or Pipe }\end{array}$} & 200 & 93 & 20.0 & 136 & 0.9 & 0.26 \\
\hline & 250 & 121 & 20.0 & 136 & 0.9 & 0.26 \\
\hline & 300 & 149 & 20.0 & 136 & 0.9 & 0.26 \\
\hline & 350 & 177 & 19.3 & 131 & 1.0 & 0.27 \\
\hline & 400 & 204 & 18.6 & 127 & 1.0 & 0.28 \\
\hline & 450 & 232 & 18.0 & 122 & 1.0 & 0.29 \\
\hline & 500 & 260 & 17.5 & 119 & 1.1 & 0.30 \\
\hline & 550 & 288 & 17.0 & 116 & 1.1 & 0.31 \\
\hline & 600 & 316 & 16.6 & 113 & 1.1 & 0.31 \\
\hline & 650 & 343 & 16.2 & 110 & 1.1 & 0.32 \\
\hline & 700 & 371 & 15.8 & 107 & 1.2 & 0.33 \\
\hline & 750 & 399 & 15.5 & 105 & 1.2 & 0.34 \\
\hline & 800 & 427 & 15.2 & 103 & 1.2 & 0.34 \\
\hline
\end{tabular}




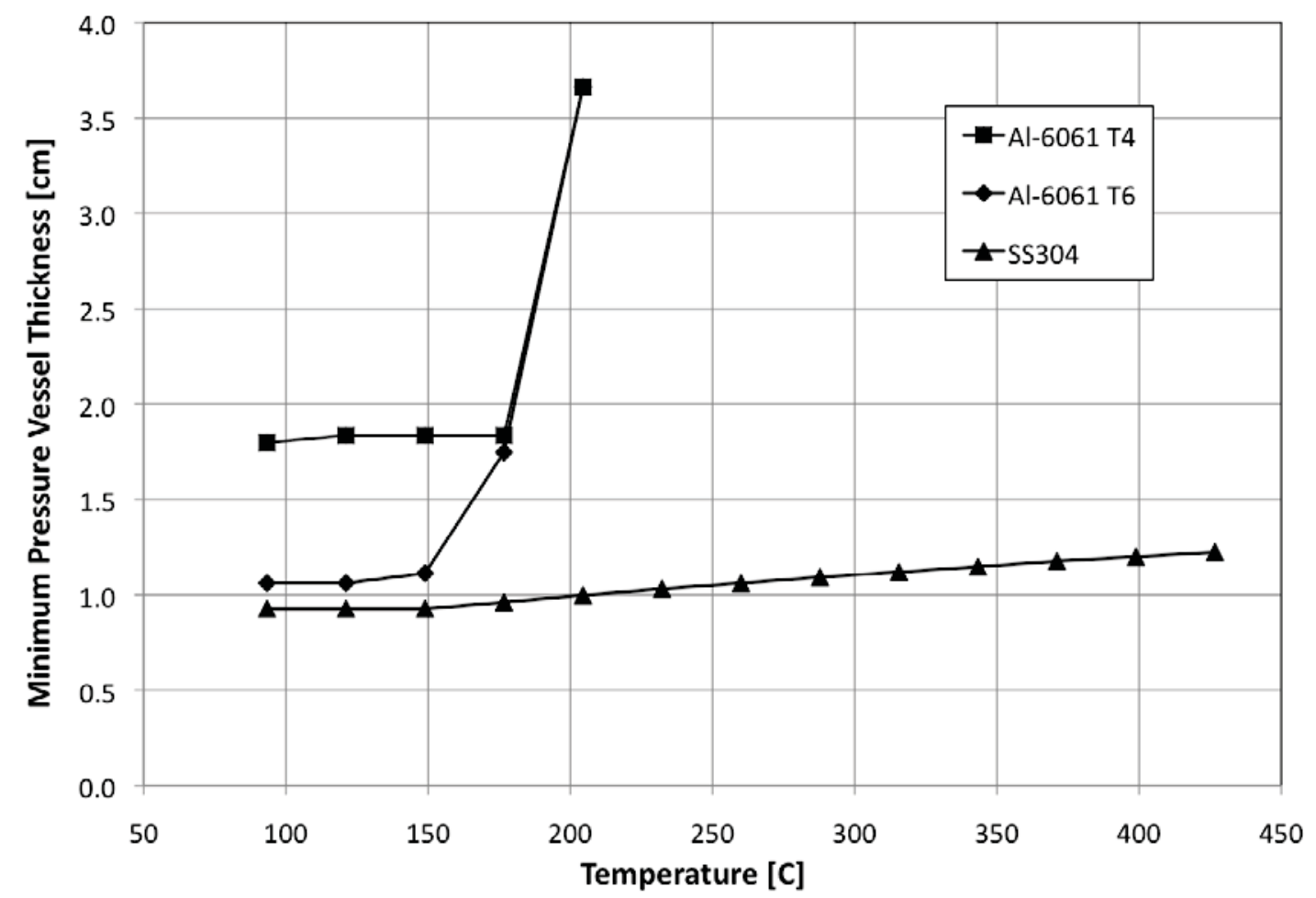

Figure 3-1. Minimum wall thickness of candidate RPV materials versus temperature for 1-meter inner diameter.

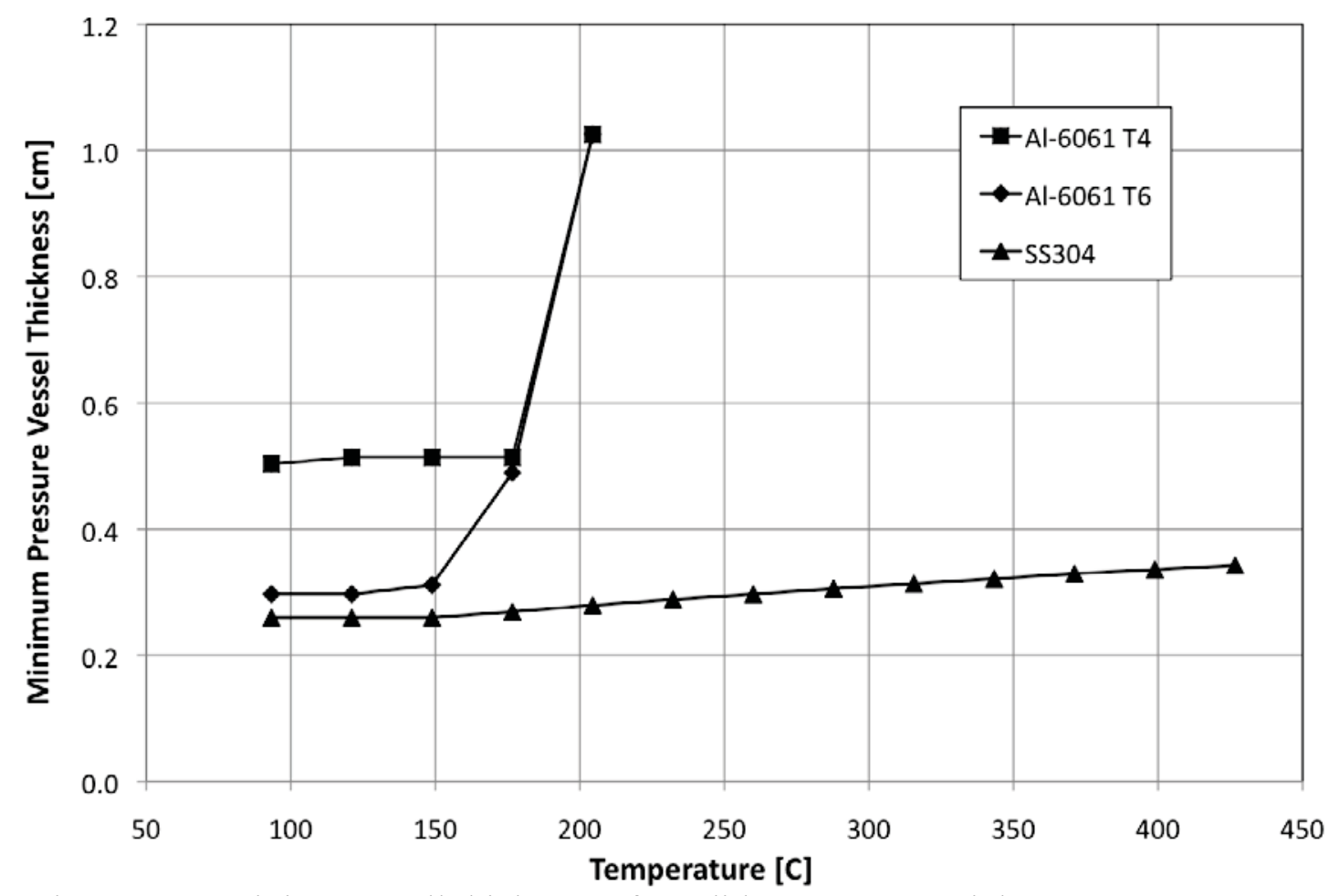

Figure 3-2. Minimum wall thickness of candidate PBT materials versus temperature for $28 \mathrm{~cm}$ diameter. 


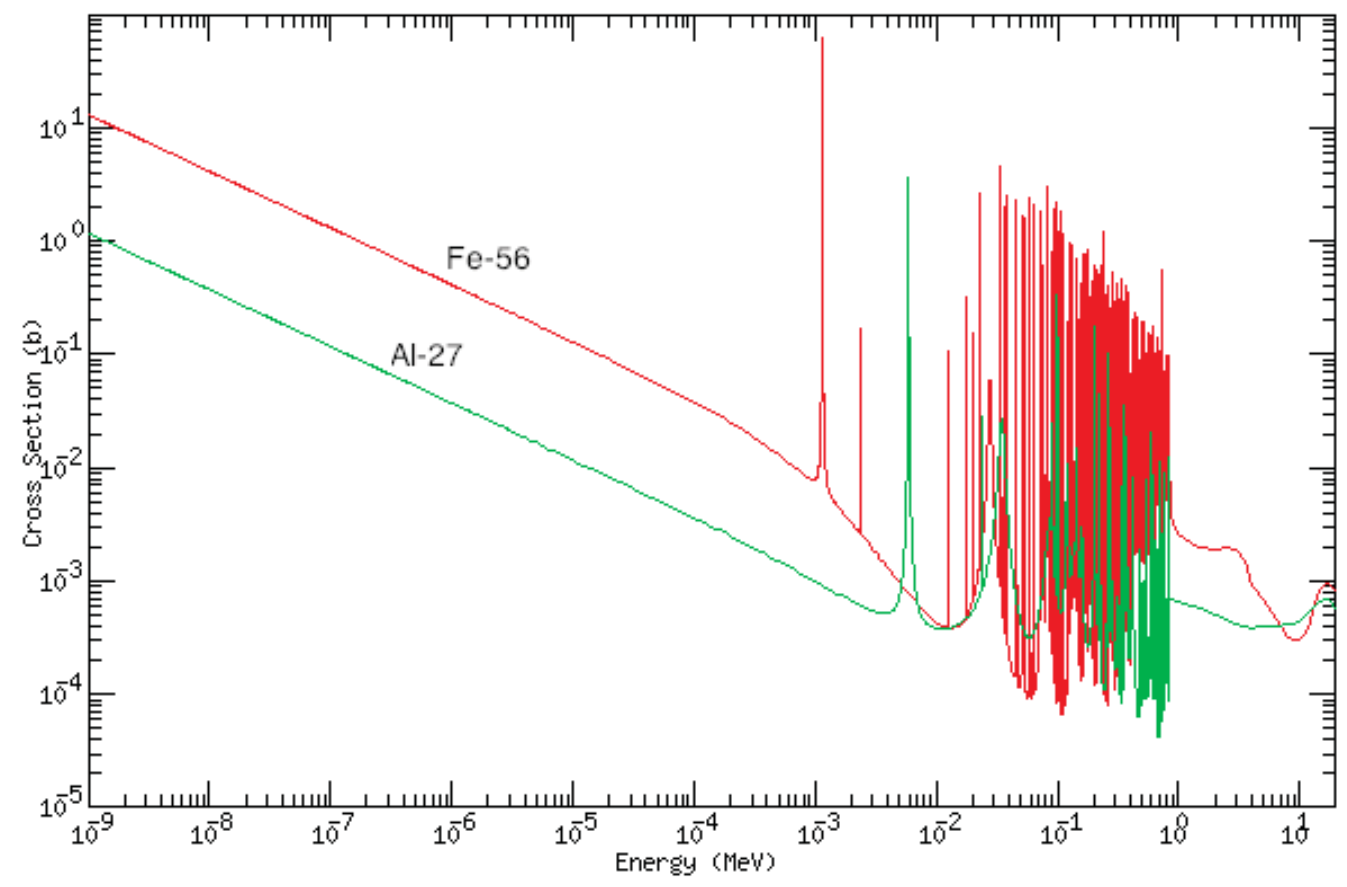

Figure 3-3. Radiative capture cross-sections of ${ }^{27} \mathrm{Al}$ and ${ }^{56} \mathrm{Fe}$. [14]

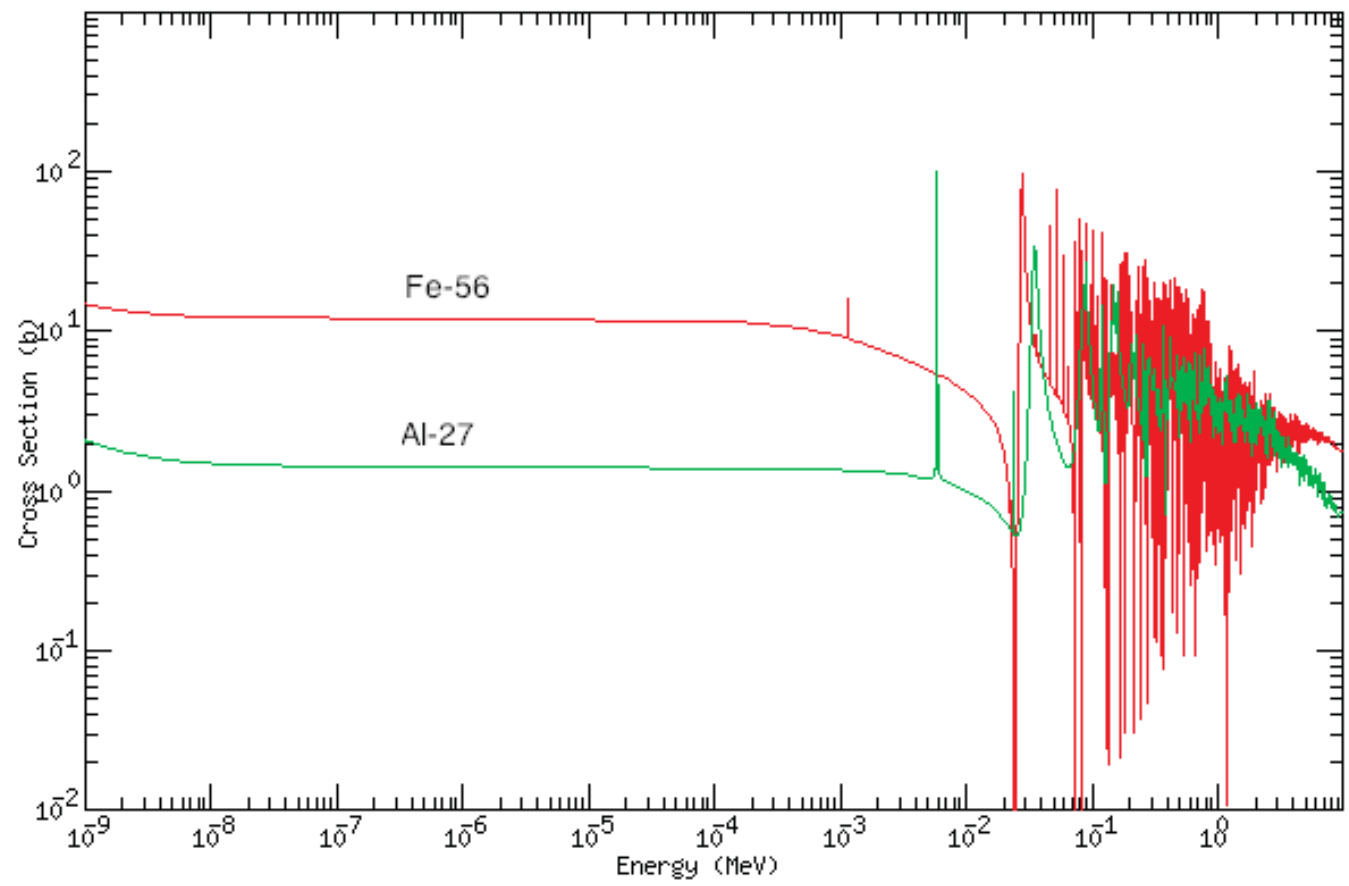

Figure 3-4. Elastic scattering cross-sections of ${ }^{27} \mathrm{Al}$ and ${ }^{56} \mathrm{Fe}$. [14] 
Table 3-2. Results of studies of SS304 pressure boundaries with comparisons to Al-6061.

\begin{tabular}{|c|c|c|c|c|c|c|}
\hline \multirow[t]{2}{*}{$\begin{array}{c}\text { Core } \\
\text { Design }\end{array}$} & \multirow[t]{2}{*}{$\begin{array}{l}\text { Pressure } \\
\text { Boundary }\end{array}$} & \multirow{2}{*}{$\begin{array}{c}\text { Initial } \\
\boldsymbol{k}_{\text {eff }}\end{array}$} & \multirow[t]{2}{*}{$\begin{array}{c}B_{1} \\
\text { (days) }\end{array}$} & \multicolumn{3}{|c|}{$\begin{array}{c}\text { Peak Neutron Flux in Reflector }\left(\mathrm{n} \cdot \mathrm{cm}^{-2} \cdot \mathrm{s}^{-1}\right) \\
\text { Fast Flux }(>1 \mathrm{MeV}) \\
\text { Thermal Flux }(<0.625 \mathrm{eV}) \\
\text { Total Flux }\end{array}$} \\
\hline & & & & $\begin{array}{l}\text { Center } \\
\text { Flux Trap }\end{array}$ & $\begin{array}{l}\text { Peripheral } \\
\text { Flux Trap }\end{array}$ & $\begin{array}{l}\text { Reflector } \\
\text { Position } 1\end{array}$ \\
\hline \multirow{9}{*}{ Cylindrical } & \multirow{3}{*}{$\begin{array}{l}\text { Al-6061 P.V. } \\
4 \mathrm{~cm} \text { thick }\end{array}$} & \multirow{3}{*}{1.18427} & \multirow{3}{*}{101} & $3.22 \mathrm{E}+14$ & $2.79 \mathrm{E}+14$ & $5.52 \mathrm{E}+12$ \\
\hline & & & & $2.77 \mathrm{E}+14$ & $2.42 \mathrm{E}+14$ & $9.82 \mathrm{E}+14$ \\
\hline & & & & $2.07 \mathrm{E}+15$ & $1.80 \mathrm{E}+15$ & $1.14 \mathrm{E}+15$ \\
\hline & \multirow{3}{*}{$\begin{array}{l}\text { S.S. } 304 \text { P.V. } \\
1.1 \mathrm{~cm} \text { thick }\end{array}$} & \multirow{3}{*}{1.13797} & \multirow{3}{*}{68} & $3.67 \mathrm{E}+14$ & $2.94 \mathrm{E}+14$ & $3.80 \mathrm{E}+12$ \\
\hline & & & & $3.07 \mathrm{E}+14$ & $2.45 \mathrm{E}+14$ & $8.46 \mathrm{E}+14$ \\
\hline & & & & $2.33 \mathrm{E}+15$ & $1.87 \mathrm{E}+15$ & $9.58 \mathrm{E}+14$ \\
\hline & \multirow{3}{*}{$\begin{array}{l}\text { Zircaloy P.V. } \\
1.1 \mathrm{~cm} \text { thick }\end{array}$} & \multirow{3}{*}{1.21359} & \multirow{3}{*}{119} & $2.93 \mathrm{E}+14$ & $2.67 \mathrm{E}+14$ & $5.81 \mathrm{E}+12$ \\
\hline & & & & $2.58 \mathrm{E}+14$ & $2.32 \mathrm{E}+14$ & $1.11 \mathrm{E}+15$ \\
\hline & & & & $1.89 \mathrm{E}+15$ & $1.71 \mathrm{E}+15$ & $1.25 \mathrm{E}+15$ \\
\hline \multirow{6}{*}{ Square } & \multirow{3}{*}{$\begin{array}{l}\text { Al-6061 P.V. } \\
4 \mathrm{~cm} \text { thick }\end{array}$} & \multirow{3}{*}{1.14066} & \multirow{3}{*}{70} & $2.71 \mathrm{E}+14$ & $2.43 \mathrm{E}+14$ & $6.83 \mathrm{E}+12$ \\
\hline & & & & $2.84 \mathrm{E}+14$ & $2.18 \mathrm{E}+14$ & $9.09 \mathrm{E}+14$ \\
\hline & & & & $2.00 \mathrm{E}+15$ & $1.62 \mathrm{E}+15$ & $1.12 \mathrm{E}+15$ \\
\hline & \multirow{3}{*}{$\begin{array}{l}\text { S.S. } 304 \text { P.V. } \\
1.1 \mathrm{~cm} \text { thick }\end{array}$} & \multirow{3}{*}{1.10069} & \multirow{3}{*}{40} & $3.09 \mathrm{E}+14$ & $2.55 \mathrm{E}+14$ & $5.07 \mathrm{E}+12$ \\
\hline & & & & $3.13 \mathrm{E}+14$ & $2.24 \mathrm{E}+14$ & $8.37 \mathrm{E}+14$ \\
\hline & & & & $2.25 \mathrm{E}+15$ & $1.68 \mathrm{E}+15$ & $9.89 \mathrm{E}+14$ \\
\hline \multirow{6}{*}{ Annular } & \multirow{3}{*}{$\begin{array}{l}\text { Al-6061 P.V. } \\
4 \mathrm{~cm} \text { thick }\end{array}$} & \multirow{3}{*}{1.13172} & \multirow{3}{*}{63} & $2.99 \mathrm{E}+14$ & $1.87 \mathrm{E}+14$ & $9.92 \mathrm{E}+12$ \\
\hline & & & & $3.57 \mathrm{E}+14$ & $2.52 \mathrm{E}+14$ & $6.45 \mathrm{E}+14$ \\
\hline & & & & $2.05 \mathrm{E}+15$ & $1.26 \mathrm{E}+15$ & $8.96 \mathrm{E}+14$ \\
\hline & \multirow{3}{*}{$\begin{array}{l}\text { S.S. } 304 \text { P.V. } \\
1.1 \mathrm{~cm} \text { thick }\end{array}$} & \multirow{3}{*}{1.10011} & \multirow{3}{*}{40} & $3.29 \mathrm{E}+14$ & $1.90 \mathrm{E}+14$ & $6.75 \mathrm{E}+12$ \\
\hline & & & & $3.87 \mathrm{E}+14$ & $2.55 \mathrm{E}+14$ & $5.77 \mathrm{E}+14$ \\
\hline & & & & $2.24 \mathrm{E}+15$ & $1.27 \mathrm{E}+15$ & $7.47 \mathrm{E}+14$ \\
\hline \multirow{6}{*}{ PBT } & \multirow{3}{*}{$\begin{array}{c}\mathrm{Al}-6061 \mathrm{PBT} \\
1.1 \mathrm{~cm} \text { thick }\end{array}$} & & & $2.99 \mathrm{E}+14$ & $1.68 \mathrm{E}+14$ & $4.75 \mathrm{E}+12$ \\
\hline & & 1.32352 & 184 & $3.57 \mathrm{E}+14$ & $2.34 \mathrm{E}+14$ & $1.14 \mathrm{E}+15$ \\
\hline & & & & $2.05 \mathrm{E}+15$ & $1.10 \mathrm{E}+15$ & $1.26 \mathrm{E}+15$ \\
\hline & & & & $2.41 \mathrm{E}+14$ & $1.68 \mathrm{E}+14$ & $4.20 \mathrm{E}+12$ \\
\hline & S.S. 304 PB & 1.22919 & 129 & $3.17 \mathrm{E}+14$ & $2.37 \mathrm{E}+14$ & $1.07 \mathrm{E}+15$ \\
\hline & & & & $1.64 \mathrm{E}+15$ & $1.10 \mathrm{E}+15$ & $1.18 \mathrm{E}+15$ \\
\hline
\end{tabular}

\footnotetext{
${ }^{a}$ For the Square core concept, the peripheral flux traps are not identical and here the North flux trap is used.
} 


\section{Reflector Tank Content}

\subsection{General Considerations for Reflector Tank Content}

The $\mathrm{D}_{2} \mathrm{O}$ reflector tank described in the baseline case descriptions in Section 2 was proposed in the BATR project as a versatile and reconfigurable region of high thermal flux at atmospheric pressure. The objective of this is to allow for various types of secondary missions to be satisfied, such as beam tubes, multiple irradiation positions, etc. These would ideally be accessible during reactor operation so as not to require additional reactor shut downs on account of reflector experiment change outs. This is the motivation for placing this region outside the main pressure boundary containing the primary coolant system. With its high moderating power, extremely low capture cross section, and liquid phase, $\mathrm{D}_{2} \mathrm{O}$ is an attractive candidate for such a reflector region. However, $\mathrm{D}_{2} \mathrm{O}$ is extremely expensive with prices ranging from $\$ 300$ to $\$ 600 \mathrm{per} \mathrm{kg}$. Furthermore, it may need to be sealed from the environment to avoid contamination by $\mathrm{H}_{2} \mathrm{O}$ in the air, partially compromising the easy accessibility afforded by its location outside the primary pressure boundary. For this reason, other reflector options were investigated for their neutronic performance. Though the possible need for sealing $\mathrm{D}_{2} \mathrm{O}$ from surrounding air in part drove this analysis, it should be noted that this requirement is not certain. In this application, some contamination of the $\mathrm{D}_{2} \mathrm{O}$ may be acceptable. Future work should evaluate this.

The alternative reflector materials evaluated here are $\mathrm{H} 2 \mathrm{O}$, beryllium, graphite, and a concept for removable, reconfigurable $\mathrm{D}_{2} \mathrm{O}$ tanks in an $\mathrm{H}_{2} \mathrm{O}$ pool. For the solid phase reflectors, some fraction of $\mathrm{H}_{2} \mathrm{O}$ cooling is assumed. Because of its high moderating power and near-zero initial cost, $\mathrm{H}_{2} \mathrm{O}$ is the most common reactor coolant/moderator in the world and is also considered a reflector in many reactors. Its main neutronic disadvantage is the high thermal neutron capture cross section $\left(664 \mathrm{mb}\right.$, mostly due to hydrogen) relative to that of $\mathrm{D}_{2} \mathrm{O}(1.33 \mathrm{mb})$, beryllium $(9.2$ $\mathrm{mb})$, and graphite $(3.4 \mathrm{mb})^{\mathrm{a}}$. This has the effect of lowering $k_{\text {eff, }}$, but also attenuating neutron flux quite sharply in a large reflector used for irradiations such as those specified for the MATRIX concepts.

Graphite is a reflector and moderator material used in many reactors throughout history. Beryllium is a reflector and moderating material used in many research reactors. This is the material used as a reflector in ATR. Despite its very attractive neutronic properties, disposal of used beryllium has proven to be difficult. As of the time of this writing, all beryllium reflector blocks that have been used in ATR remain in the spent fuel canal. Both beryllium and graphite are analyzed as pure having $10 \%$ by volume $\mathrm{H}_{2} \mathrm{O}$ coolant homogeneously mixed into them. This gives a rough approximation of the neutronic impact of cooling them, albeit an exaggerated one since homogeneous mixing of the water can increase its impact over discrete cooling channels.

\subsection{Replaceable, Reconfigurable $\mathrm{D}_{2} \mathrm{O}$ Tanks}

Another reflector configuration to be investigated here is that of replaceable, reconfigurable $\mathrm{D}_{2} \mathrm{O}$ tanks in a water pool. This is proposed as a compromise between the low initial cost and open access to a water pool with the low capture cross section of $\mathrm{D}_{2} \mathrm{O}$. Figure 4-1 shows schematics of what this concept could look like. The above view shows that the tanks surround

${ }^{\text {a }} 0.0253 \mathrm{eV}$ values from Lamarsh \& Baratta, Introduction to Nuclear Engineering, $3^{\text {rd }}$ Edition, 2001. 
the core and pressure boundary in eight segments, each subtending a $45^{\circ}$ angle. The tanks are constructed from Al-6061 and are here assumed to have $2 \mathrm{~cm}$ thick walls. These tank walls will bear minimal load since the inside and outside pressures are nearly atmospheric. Test positions could be machined into the tanks such that the $\mathrm{D}_{2} \mathrm{O}$ is sealed inside and experiments could be raised and lowered out of their irradiation positions from above the tank without shutting down the reactor and without penetrating the $\mathrm{D}_{2} \mathrm{O}$-filled chamber. Figure 4-1 shows optional piping that could be used for $\mathrm{D}_{2} \mathrm{O}$ cleanup, such as tritium removal, online. However, since these tanks would be removable, it may be more attractive to leave the tanks sealed with space for gases to accumulate and process the tanks periodically outside the core.

Figure 4-2 shows an above cross sectional view of the baseline Cylindrical core model with the removable, reconfigurable $\mathrm{D}_{2} \mathrm{O}$ tanks positioned outside the pressure boundary as modeled in Serpent. The clearance between the $\mathrm{D}_{2} \mathrm{O}$ tanks is $1.0 \mathrm{~cm}$ and that between the pressure vessel outer surface and the $\mathrm{D}_{2} \mathrm{O}$ tank wall is $0.5 \mathrm{~cm}$. A distance of $8.0 \mathrm{~cm}$ is selected rather arbitrarily as the clearance between the outer $\mathrm{D}_{2} \mathrm{O}$ tank surface and the boundary of the $\mathrm{H}_{2} \mathrm{O}$ pool. Figure 4-3 shows a vertical cross section view of the same Serpent model. This shows that the $\mathrm{D}_{2} \mathrm{O}$ tanks were conceptualized such that they extend $10 \mathrm{~cm}$ above and below the active core height of $120 \mathrm{~cm}$. The baseline Cylindrical model described in Section 2.1 has a large $\mathrm{D}_{2} \mathrm{O}$ reflector tank with a volume of approximately $9.5 \mathrm{~m}^{3}$. This amounts to $10,500 \mathrm{~kg}$ of $\mathrm{D}_{2} \mathrm{O}$, and given the price range above, a capital cost of approximately $\$ 3$ to $\$ 6$ million. The removable reflector tanks described here and shown in Figure 4-2 and Figure 4-3 would reduce the amount of $\mathrm{D}_{2} \mathrm{O}$ required by roughly a factor of two.

Figure 4-4 shows an above view of the neutron flux in the Cylindrical core at BOL. The white areas represent regions of high thermal flux, while blue areas represent high fast flux. The yellow and red regions represent higher and lower fission power, respectively. The thermal neutron flux is observed to be reduced somewhat in the gaps between the $\mathrm{D}_{2} \mathrm{O}$ tanks where $\mathrm{H}_{2} \mathrm{O}$ is present. Figure $4-5$ shows a flux plot of the same case in an axial cross section view. 


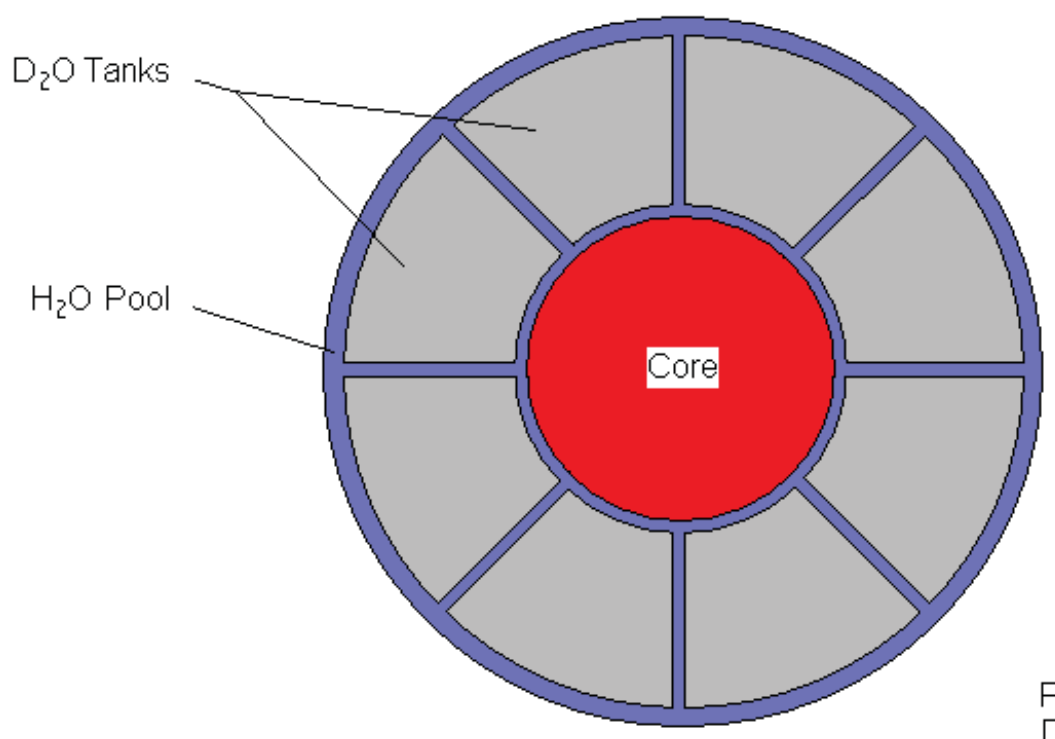

Possible piping for $\mathrm{D}_{2} \mathrm{O}$ chemistry and

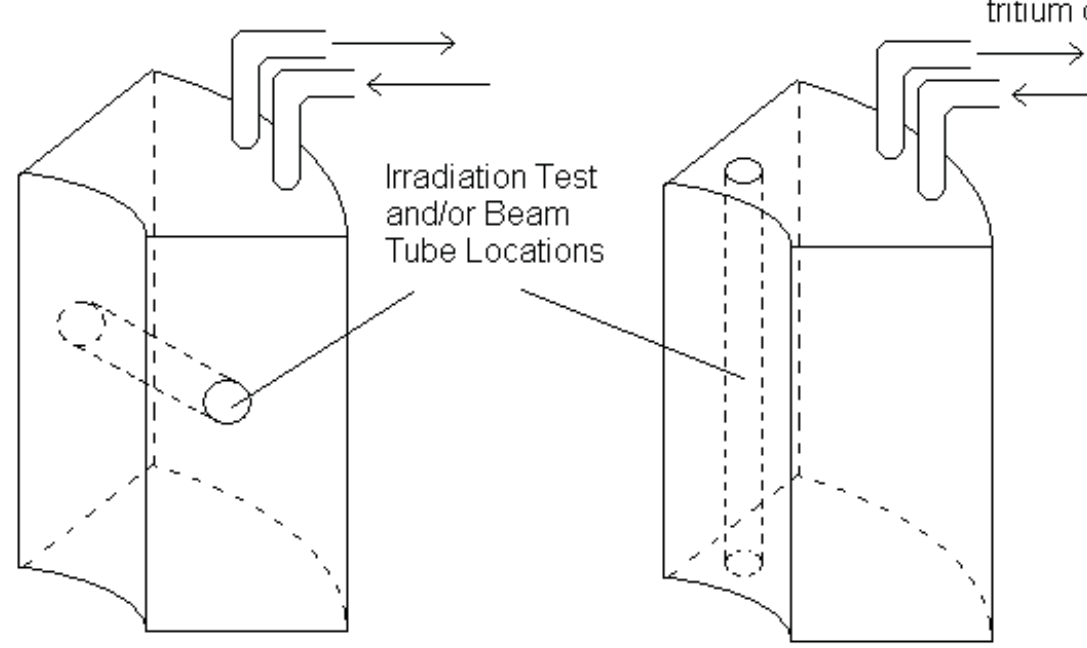

Figure 4-1. Sketches of removable, reconfigurable $\mathrm{D}_{2} \mathrm{O}$ reflector tanks. 


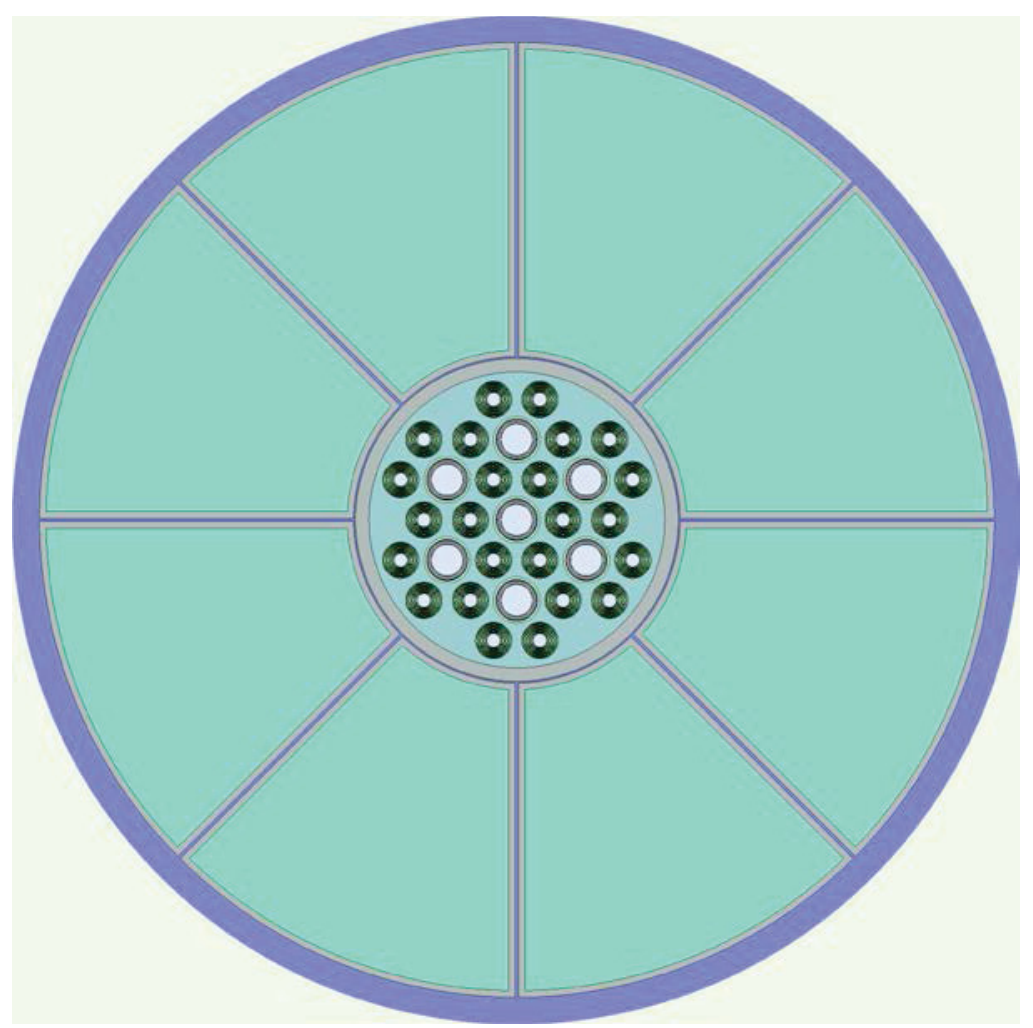

Figure 4-2. Above view of cylindrical core model with $\mathrm{D}_{2} \mathrm{O}$ tanks at axial core center.

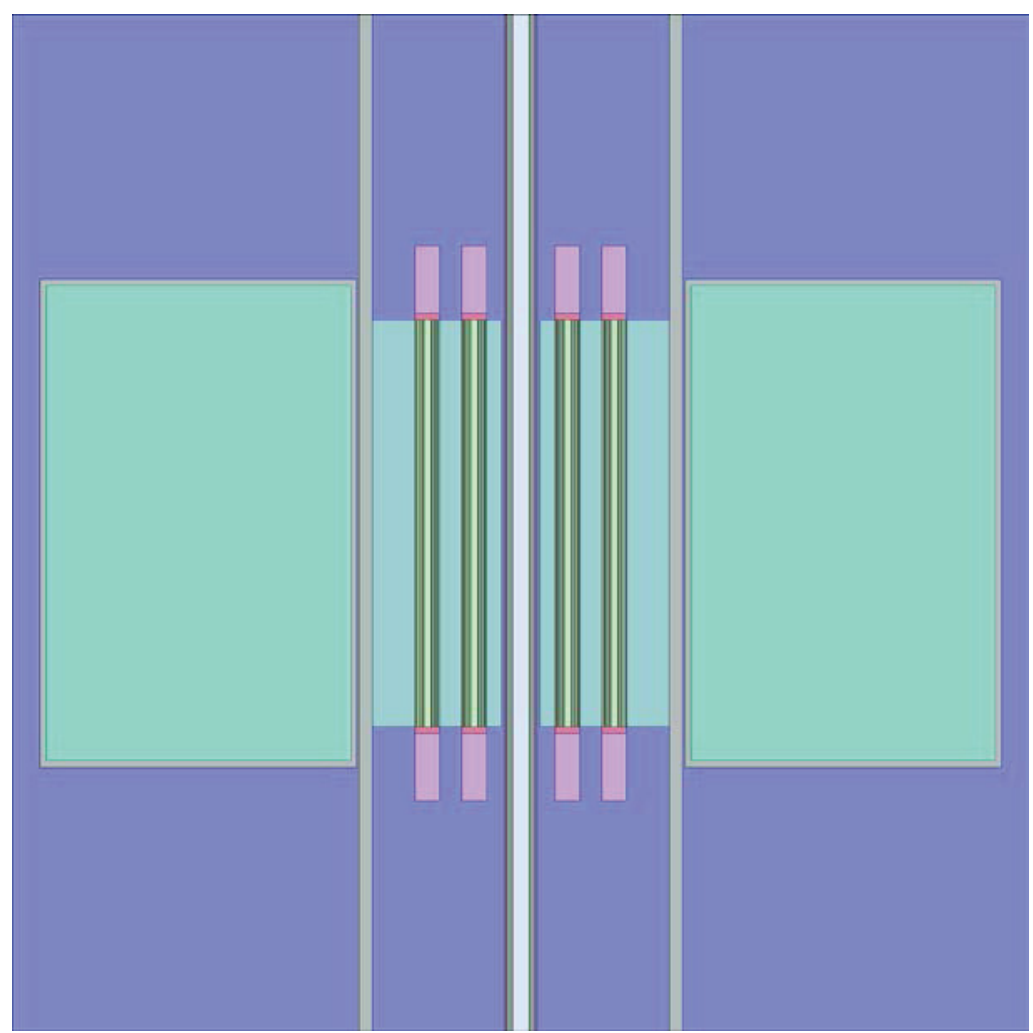

Figure 4-3. Side view of cylindrical core model with $\mathrm{D}_{2} \mathrm{O}$ tanks at axial core center. 


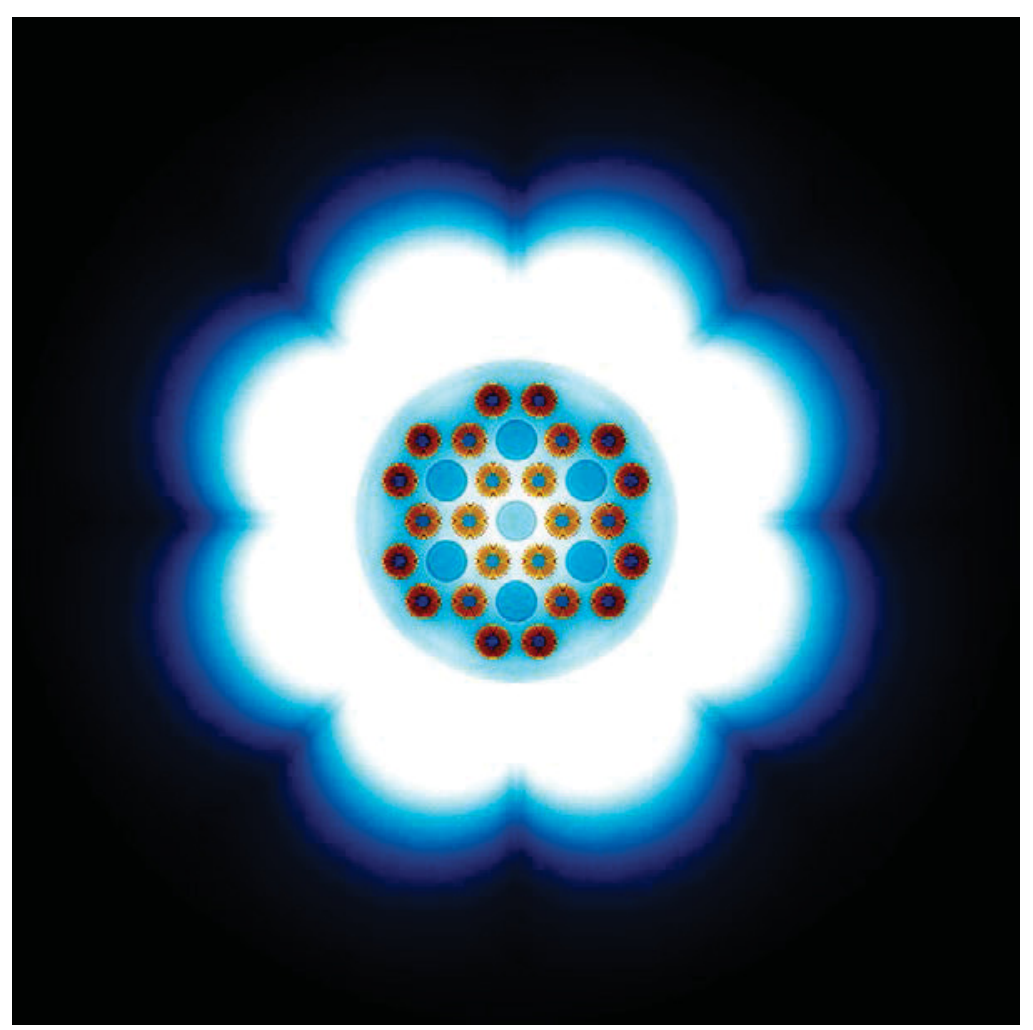

Figure 4-4. Above view flux plot of cylindrical core with $\mathrm{D}_{2} \mathrm{O}$ reflector tanks.

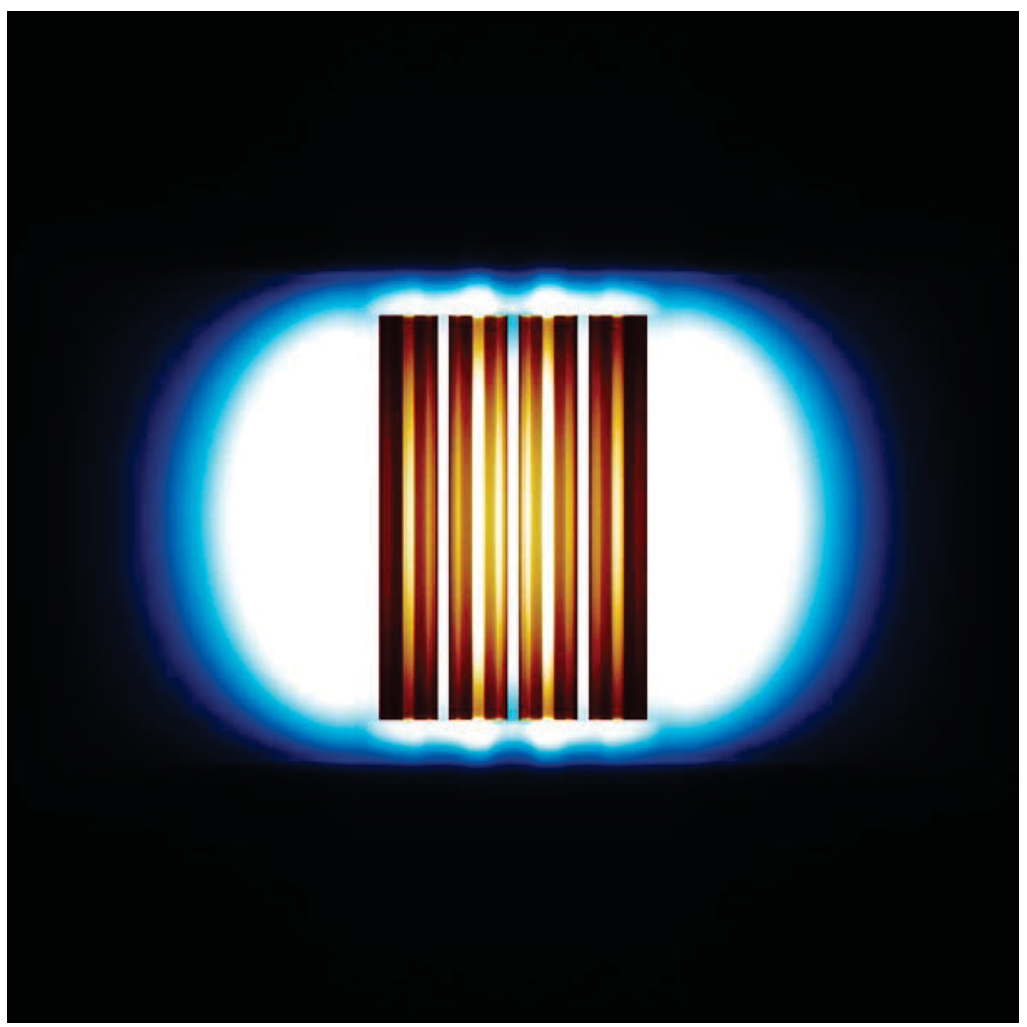

Figure 4-5. Side view flux plot of cylindrical core with $\mathrm{D}_{2} \mathrm{O}$ reflector tanks. 


\subsection{Performance Comparison}

Serpent cases were generated comparing seven different reflector tank contents for the otherwise baseline Cylindrical reactor. The baseline case of $100 \% \mathrm{D}_{2} \mathrm{O}$ is repeated here for comparison. The six other cases were $100 \%$ graphite, graphite with $10 \% \mathrm{H}_{2} \mathrm{O}, 100 \%$ beryllium, beryllium with $10 \% \mathrm{H}_{2} \mathrm{O}$, the removable $\mathrm{D}_{2} \mathrm{O}$ tanks described above, the same removable $\mathrm{D}_{2} \mathrm{O}$ tanks with $10 \%$ water in the $\mathrm{D}_{2} \mathrm{O}$, and $100 \% \mathrm{H}_{2} \mathrm{O}$. Figure 4-6 shows the peak (axially centered) thermal neutron flux versus distance from the outer surface of the pressure vessel. As expected, the reflector material that preserves the highest flux versus distance from the vessel is $\mathrm{D}_{2} \mathrm{O}$, with a thermal flux in excess of $10^{14} \mathrm{n} \cdot \mathrm{cm}^{-2} \cdot \mathrm{s}^{-1}$ for essentially the entire distance to the outer reflector tank wall. This was followed by $100 \%$ graphite, and then by the $\mathrm{D}_{2} \mathrm{O}$ tanks in a water pool. The case with $100 \%$ beryllium had the next highest flux far from the pressure vessel (though close to the vessel, it had higher thermal flux than the $100 \%$ graphite and $\mathrm{D}_{2} \mathrm{O}$ tanks cases). The worst case by far with regard to flux in the reflector tank was the $100 \% \mathrm{H}_{2} \mathrm{O}$ case.

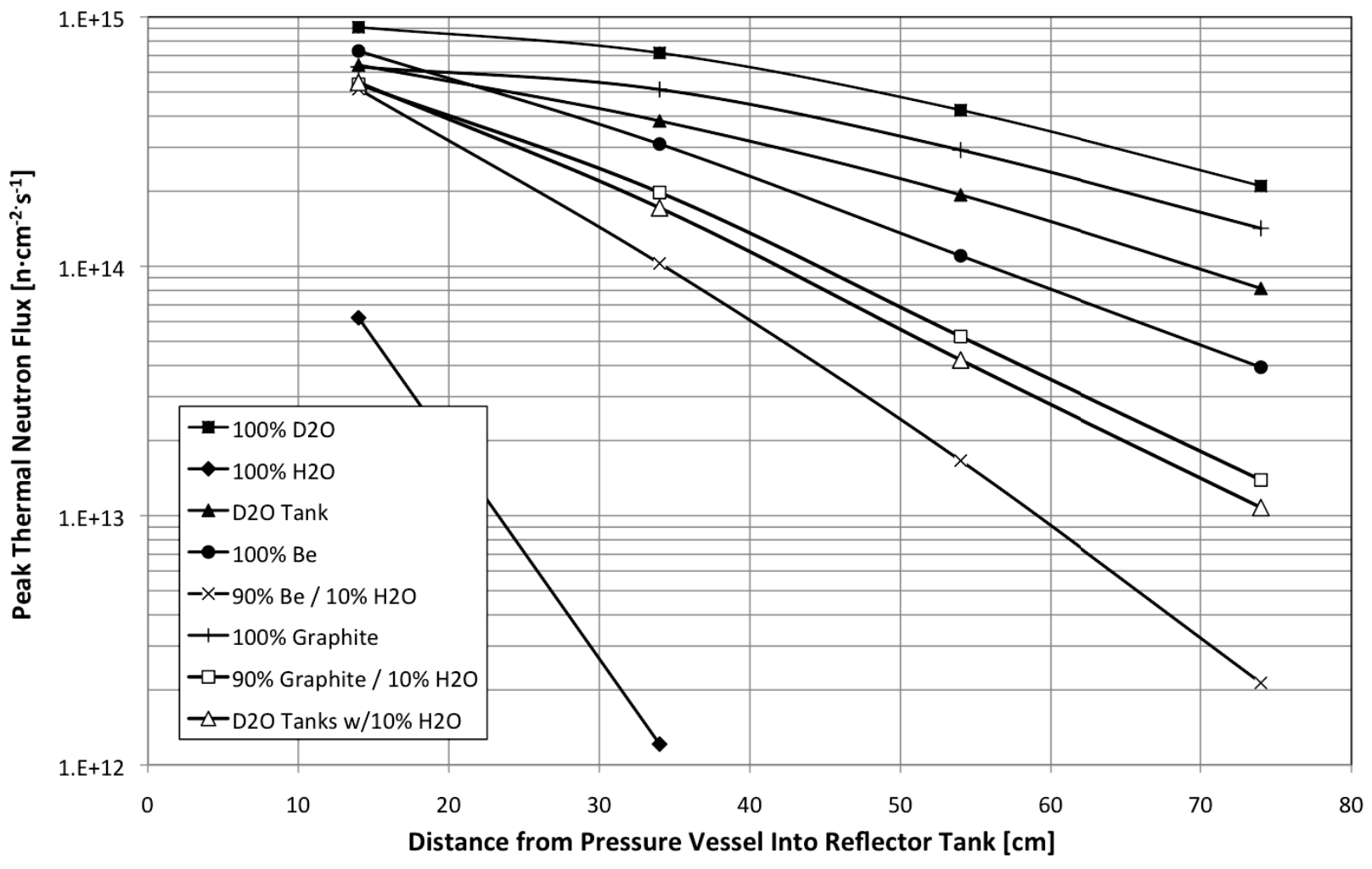

Figure 4-6. Peak thermal flux (at axial centerline) in reflector versus distance from pressure vessel with various reflector tank contents in cylindrical core.

Table 4-1 shows the initial $k_{\text {eff }}$ and $B_{1}$ values calculated for the cylindrical core with the various contents of the reflector tank region. The general trend is that the reflectors which give greatest overall thermal flux in the reflector tank also yield the highest $k_{\text {eff. }}$. However, this trend is not absolute, as some materials are better reflectors, but may absorb more neutrons than other materials. An example is the case of $100 \%$ beryllium, which gives the highest initial $k_{\text {eff }}$ of any 
of the materials examined here. However, it does not have the highest thermal flux in the reflector region (though the slope of its curve in Figure 4-6 indicates that it may indeed have the highest thermal flux very close to the pressure vessel. Though beryllium is acting as the best reflector with regard to neutron reflection, it has greater absorption than some of the other candidate materials. Therefore, it may not be the best material for extending the thermal flux to as great a distance from the pressure vessel as possible. Also seen in Table 4-1 is $\mathrm{H}_{2} \mathrm{O}$ performing worse than any of the other candidate reflectors with regard to both initial $k_{\text {eff }}$ and $B_{1}$. Figure 4-7 shows the radiative capture cross sections of four of the key nuclides in these reflector materials. This shows that ${ }^{2} \mathrm{H}$ has a much lower capture cross-section than ${ }^{1} \mathrm{H}$, and that ${ }^{12} \mathrm{C}$ has a lower capture cross-section than ${ }^{9} \mathrm{Be}$. This gives a heuristic explanation for the performance of these materials in this application.

Table 4-1. Reactivity performance of cylindrical core with various reflector contents.

\begin{tabular}{|l|c|c|}
\hline Reflector Tank Contents & Initial $\boldsymbol{k}_{\text {eff }}$ & $\begin{array}{c}\boldsymbol{B}_{\boldsymbol{1}} \\
\text { (days) }\end{array}$ \\
\hline $\mathrm{D}_{2} \mathrm{O}$ & 1.18427 & 100 \\
\hline $\mathrm{H}_{2} \mathrm{O}$ & 1.10190 & 42 \\
\hline $\mathrm{D}_{2} \mathrm{O}$ Tanks in $\mathrm{H}_{2} \mathrm{O}$ & 1.13833 & 69 \\
\hline $100 \%$ Beryllium & 1.19594 & 108 \\
\hline $90 \%$ Beryllium $/ 10 \% \mathrm{H}_{2} \mathrm{O}$ & 1.17034 & 91 \\
\hline $100 \%$ Graphite & 1.19133 & 105 \\
\hline $90 \%$ Graphite $/ 10 \% \mathrm{H}_{2} \mathrm{O}$ & 1.15750 & 82 \\
\hline $\mathrm{D} 2 \mathrm{O}$ Tanks with $10 \% \mathrm{H}_{2} \mathrm{O}$ in $\mathrm{D}_{2} \mathrm{O}$ & 1.12587 & 60 \\
\hline
\end{tabular}




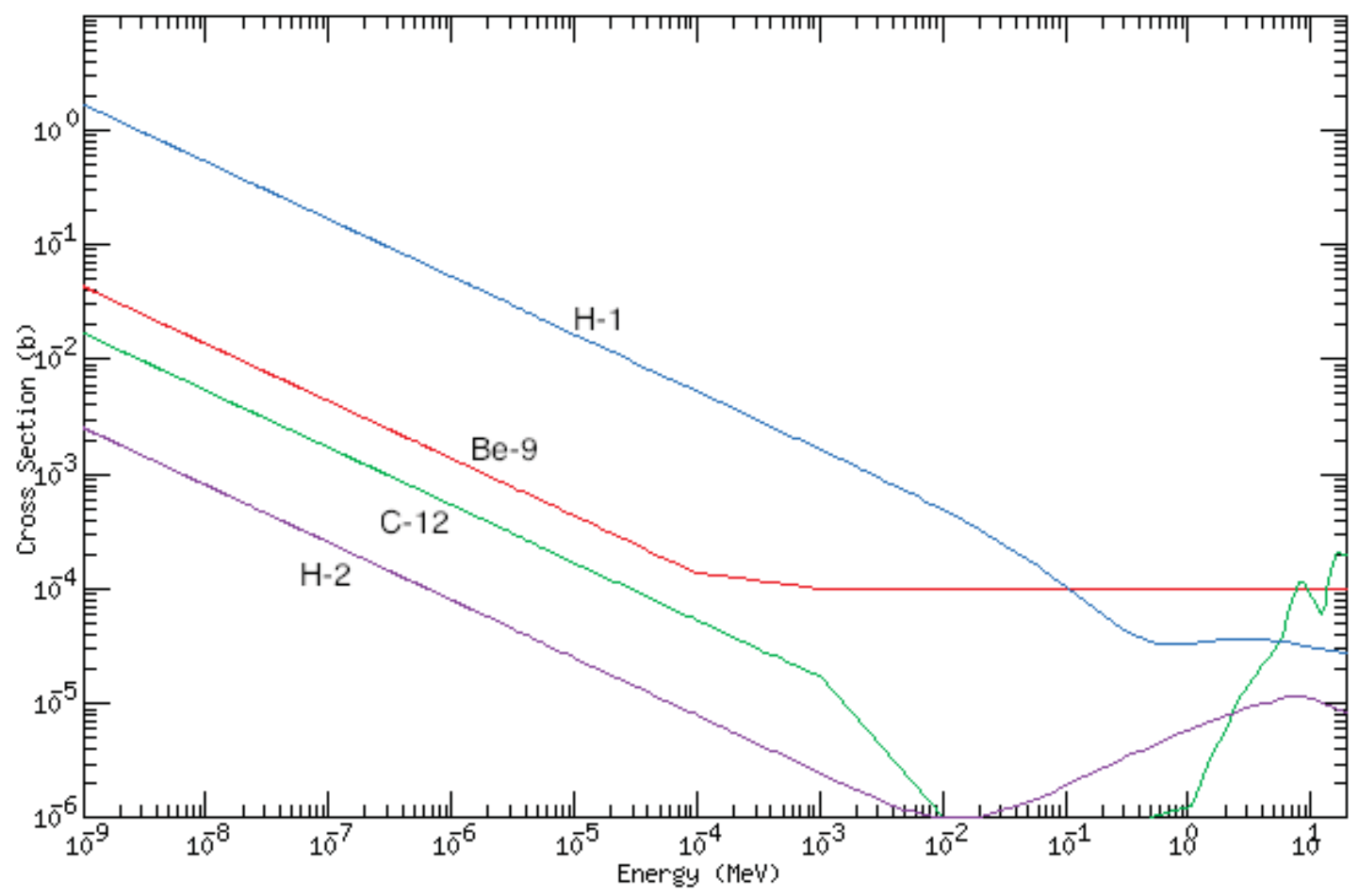

Figure 4-7. Radiative capture cross-sections of ${ }^{1} \mathrm{H}$ and ${ }^{2} \mathrm{H},{ }^{12} \mathrm{C}$, and ${ }^{9} \mathrm{Be}$. [14]

\section{Core Rack Content Options}

Three of the baseline reactor concepts introduced in Section 2 specify an aluminum core rack cooled by an assumed $6.3 \%$ by volume coolant $\mathrm{H}_{2} \mathrm{O}$. This configuration is meant to allow for maintaining a high fast flux, which can be thermalized if desired. It also avoids the use of beryllium, which suffers from embrittlement under irradiation that limits its lifetime and carries a toxicity which poses a challenge to disposal. [16]. Furthermore, because the aluminum rack does not moderate neutrons effectively, it contributes to a rather low reactivity core. For example, a core with a beryllium rack and the same fuel would have a much higher reactivity. This has implications toward achievable cycle length and the feasibility of using alternative fuel forms with lower heavy metal (HM) density than U-10Mo. This will be discussed further in Section 6. As an alternative to the aluminum rack, it has been proposed that aluminum/beryllium alloys be considered for this application. Adding beryllium content affects the neutronic characteristics of the core by 1) increasing reactivity, 2) reducing fast flux in test positions, and 2) reducing fast fluence on the pressure vessel.

Serpent calculations were performed on the baseline Cylindrical and Annular core designs to quantify the effects of different core rack contents. The cases examined are shown in Table 5-1 along with a Rack ID, which is used in the subsequent tables for identifying the content of the core rack in each case. Beryllium was varied from 0 to $93.7 \%$ with $\mathrm{H}_{2} \mathrm{O}$ assumed to occupy $6.3 \%$ by volume. 
Table 5-1. Core rack contents evaluated identified by a Rack ID.

\begin{tabular}{|l|c|c|c|}
\hline Rack ID & Vol. \% Aluminum & Vol. \% beryllium & \multirow{2}{*}{ Vol. \% $\mathbf{H}_{2} \mathbf{O}$} \\
\hline Be- 0 & 93.7 & 0 & \multirow{2}{*}{6} \\
\cline { 1 - 3 }-20 & 73.7 & 20 & \multirow{2}{*}{6.3} \\
\hline Be-50 & 43.7 & 50 & \\
\hline Be-100 & 0 & 93.7 & \\
\hline
\end{tabular}

Results of calculations carried out on the Annular core are given in Table 5-1. The table shows $k_{\text {eff }}$ and $B_{1}$ along with neutron flux in three different locations; center flux trap, peripheral flux trap, and Reflector Tally Position \#1 (see Figure 2-18 on page 31). From the table, one also sees that the reduction in fast flux as a result of increased beryllium content in the rack is minimal in the center and peripheral flux traps. On the other hand, the thermal flux in the flux trap is roughly doubled in going from the Be- 0 case to the Be-100 case. Going from all aluminum to all beryllium core rack material also increases the thermal flux in Reflector position \#1 slightly, though it does decrease total flux somewhat. Meanwhile, the initial $k_{\text {eff }}$ has increased from 1.13 to 1.32 , offering quite a bit of flexibility in design such as the option of reducing HM loading, having longer cycle lengths, etc. The $B_{1}$ values increased from 63 days in the baseline $\mathrm{Be}-0$ case to 176 days in the Be-100 case. Also of note is the somewhat lower axial peaking in fast flux in the flux traps for the aluminum rack than the beryllium rack. The aluminum rack case has lower axial peaking because it has moderation concentrated at the top and bottom from cooling water with less moderation along the length of the fuel. The beryllium rack provides moderation along its length, so a more peaked shape results.

Results of calculations carried out on the Cylindrical core are given in Table 5-3 in a similar format to Table 5-2. The basic trends are the same as with the Annular core. Here, the additional location in a fuel assembly center is available. From this point, one sees how the increased moderation in the Be-100 case reduces the thermal flux substantially from the baseline Be-0 case. This is because the more thermal spectrum results in higher effective fission crosssections in the fuel and a lower thermal flux for a given power. The initial $k_{\text {eff }}$ has increased from 1.18 to 1.28 in moving from the $\mathrm{Be}-0$ to the $\mathrm{Be}-100$ case while the $B_{1}$ values increased from 100 days to 165 days.

Table 5-4 gives neutron fluence rates at the inner surface of the pressure vessel at the axial center for the Cylindrical and Annular cases discussed above. These results show that replacing the Be-0 rack with the Be-100 rack reduces the fast fluence in the pressure vessel by approximately a factor of 2 while only slightly decreasing the total fluence. Since most of the neutron damage is caused by fast neutrons, this may have important implications on the frequency of pressure vessel replacement. 
Table 5-2. Performance parameters for Annular core with varying rack contents.

\begin{tabular}{|c|c|c|c|c|}
\hline \multicolumn{2}{|c|}{$\begin{array}{c}\text { Core Rack Content and } \\
\text { Tally Location }\end{array}$} & \multicolumn{3}{|c|}{$\begin{array}{c}\text { Neutron Flux }\left(\mathrm{n} \cdot \mathrm{cm}^{-2} \cdot \mathrm{s}^{-1}\right) \\
\text { Fast Flux }(>1 \mathrm{MeV}) \\
\text { Thermal Flux }(<0.625 \mathrm{eV}) \\
\text { Total Flux }\end{array}$} \\
\hline $\begin{array}{c}\text { Core Rack ID } \\
k_{e f f} \text { and } B_{1}\end{array}$ & $\begin{array}{c}\text { Tally } \\
\text { Location }\end{array}$ & Peak & $\begin{array}{c}\text { Axially } \\
\text { Averaged }\end{array}$ & $\begin{array}{c}\text { Peak/ } \\
\text { Average }\end{array}$ \\
\hline \multirow{4}{*}{ Be-0 } & \multirow{3}{*}{$\begin{array}{c}\text { Center Flux } \\
\text { Trap }\end{array}$} & $3.05 \mathrm{E}+14$ & $2.30 \mathrm{E}+14$ & 1.33 \\
\hline & & $3.57 \mathrm{E}+14$ & $2.74 \mathrm{E}+14$ & 1.30 \\
\hline & & $2.07 \mathrm{E}+15$ & $1.56 \mathrm{E}+15$ & 1.32 \\
\hline & \multirow{3}{*}{$\begin{array}{c}\text { Peripheral Flux } \\
\text { Trap }\end{array}$} & $1.87 \mathrm{E}+14$ & $1.43 \mathrm{E}+14$ & 1.31 \\
\hline \multirow{5}{*}{$\begin{array}{c}\text { Initial } k_{\text {eff }}=1.13172 \\
B_{l}=63 \text { days }\end{array}$} & & $2.45 \mathrm{E}+14$ & $1.91 \mathrm{E}+14$ & 1.29 \\
\hline & & $1.24 \mathrm{E}+15$ & $9.48 \mathrm{E}+14$ & 1.31 \\
\hline & \multirow{3}{*}{$\begin{array}{l}\text { Reflector Tally } \\
\text { Position } 1\end{array}$} & $1.01 \mathrm{E}+13$ & $7.37 \mathrm{E}+12$ & 1.37 \\
\hline & & $6.75 \mathrm{E}+14$ & $5.39 \mathrm{E}+14$ & 1.25 \\
\hline & & $9.19 \mathrm{E}+14$ & $7.22 \mathrm{E}+14$ & 1.27 \\
\hline \multirow{9}{*}{$\begin{array}{c}\text { Initial } \\
k_{e f f}=1.17019 \\
B_{1}=89 \text { days }\end{array}$} & \multirow{3}{*}{$\begin{array}{c}\text { Center Flux } \\
\text { Trap }\end{array}$} & $3.10 \mathrm{E}+14$ & $2.28 \mathrm{E}+14$ & 1.36 \\
\hline & & $3.71 \mathrm{E}+14$ & $2.80 \mathrm{E}+14$ & 1.32 \\
\hline & & $2.10 \mathrm{E}+15$ & $1.56 \mathrm{E}+15$ & 1.34 \\
\hline & \multirow{3}{*}{$\begin{array}{c}\text { Peripheral Flux } \\
\text { Trap }\end{array}$} & $1.83 \mathrm{E}+14$ & $1.39 \mathrm{E}+14$ & 1.32 \\
\hline & & $2.55 \mathrm{E}+14$ & $1.91 \mathrm{E}+14$ & 1.34 \\
\hline & & $1.25 \mathrm{E}+15$ & $9.32 \mathrm{E}+14$ & 1.34 \\
\hline & \multirow{3}{*}{$\begin{array}{l}\text { Reflector Tally } \\
\text { Position } 1\end{array}$} & $7.11 \mathrm{E}+12$ & $6.28 \mathrm{E}+12$ & 1.13 \\
\hline & & $6.33 \mathrm{E}+14$ & $5.17 \mathrm{E}+14$ & 1.22 \\
\hline & & $8.37 \mathrm{E}+14$ & $6.69 \mathrm{E}+14$ & 1.25 \\
\hline \multirow{9}{*}{$\begin{array}{c}\text { Initial } \\
k_{e f f}=1.22543 \\
B_{1}=124 \text { days }\end{array}$} & \multirow{3}{*}{$\begin{array}{l}\text { Center Flux } \\
\text { Trap }\end{array}$} & $2.98 \mathrm{E}+14$ & $2.20 \mathrm{E}+14$ & 1.36 \\
\hline & & $3.88 \mathrm{E}+14$ & $2.87 \mathrm{E}+14$ & 1.35 \\
\hline & & $2.08 \mathrm{E}+15$ & $1.53 \mathrm{E}+15$ & 1.36 \\
\hline & \multirow{3}{*}{$\begin{array}{c}\text { Peripheral Flux } \\
\text { Trap }\end{array}$} & $1.83 \mathrm{E}+14$ & $1.35 \mathrm{E}+14$ & 1.36 \\
\hline & & $2.59 \mathrm{E}+14$ & $1.90 \mathrm{E}+14$ & 1.37 \\
\hline & & $1.24 \mathrm{E}+15$ & $9.11 \mathrm{E}+14$ & 1.36 \\
\hline & \multirow{3}{*}{$\begin{array}{l}\text { Reflector Tally } \\
\text { Position } 1\end{array}$} & $5.95 \mathrm{E}+12$ & $4.70 \mathrm{E}+12$ & 1.27 \\
\hline & & $6.30 \mathrm{E}+14$ & $4.96 \mathrm{E}+14$ & 1.27 \\
\hline & & $7.97 \mathrm{E}+14$ & $6.18 \mathrm{E}+14$ & 1.29 \\
\hline \multirow{9}{*}{ Be-100 } & \multirow{3}{*}{$\begin{array}{l}\text { Center Flux } \\
\text { Trap }\end{array}$} & $2.70 \mathrm{E}+14$ & $1.99 \mathrm{E}+14$ & 1.36 \\
\hline & & $3.77 \mathrm{E}+14$ & $2.73 \mathrm{E}+14$ & 1.38 \\
\hline & & $1.94 \mathrm{E}+15$ & $1.40 \mathrm{E}+15$ & 1.38 \\
\hline & \multirow{3}{*}{$\begin{array}{l}\text { Peripheral Flux } \\
\text { Trap }\end{array}$} & $1.76 \mathrm{E}+14$ & $1.29 \mathrm{E}+14$ & 1.36 \\
\hline & & $2.54 \mathrm{E}+14$ & $1.88 \mathrm{E}+14$ & 1.36 \\
\hline & & $1.21 \mathrm{E}+15$ & $8.86 \mathrm{E}+14$ & 1.36 \\
\hline & \multirow{3}{*}{$\begin{array}{l}\text { Reflector Tally } \\
\text { Position } 1\end{array}$} & $4.20 \mathrm{E}+12$ & $3.42 \mathrm{E}+12$ & 1.23 \\
\hline & & $6.53 \mathrm{E}+14$ & $5.01 \mathrm{E}+14$ & 1.30 \\
\hline & & $7.74 \mathrm{E}+14$ & $5.90 \mathrm{E}+14$ & 1.31 \\
\hline
\end{tabular}


Table 5-3. Performance parameters for Cylindrical core with varying rack contents.

\begin{tabular}{|c|c|c|c|c|}
\hline \multicolumn{2}{|c|}{$\begin{array}{c}\text { Core Rack Content and } \\
\text { Tally Location }\end{array}$} & \multicolumn{3}{|c|}{$\begin{array}{c}\text { Neutron Flux }\left(\mathrm{n} \cdot \mathrm{cm}^{-2} \cdot \mathrm{s}^{-1}\right) \\
\text { Fast Flux }(>1 \mathrm{MeV}) \\
\text { Thermal Flux }(<0.625 \mathrm{eV}) \\
\text { Total Flux } \\
\end{array}$} \\
\hline $\begin{array}{c}\text { Core Rack ID } \\
k_{\text {eff }} \text { and } B_{l}\end{array}$ & $\begin{array}{c}\text { Tally } \\
\text { Location }\end{array}$ & Peak & $\begin{array}{c}\text { Axially } \\
\text { Averaged }\end{array}$ & $\begin{array}{c}\text { Peak/ } \\
\text { Average }\end{array}$ \\
\hline \multirow{12}{*}{$\mathrm{Be}-0$} & \multirow{3}{*}{ Center Flux Trap } & $3.21 \mathrm{E}+14$ & $2.42 \mathrm{E}+14$ & 1.33 \\
\hline & & $2.87 \mathrm{E}+14$ & $2.16 \mathrm{E}+14$ & 1.32 \\
\hline & & $2.07 \mathrm{E}+15$ & $1.55 \mathrm{E}+15$ & 1.33 \\
\hline & \multirow{3}{*}{$\begin{array}{c}\text { Peripheral Flux } \\
\text { Trap }\end{array}$} & $2.78 \mathrm{E}+14$ & $2.09 \mathrm{E}+14$ & 1.33 \\
\hline & & $2.50 \mathrm{E}+14$ & $1.90 \mathrm{E}+14$ & 1.32 \\
\hline & & $1.79 \mathrm{E}+15$ & $1.35 \mathrm{E}+15$ & 1.32 \\
\hline & \multirow{3}{*}{$\begin{array}{l}\text { Inner Fuel } \\
\text { Assembly }\end{array}$} & $5.20 \mathrm{E}+14$ & $3.91 \mathrm{E}+14$ & 1.33 \\
\hline & & $1.42 \mathrm{E}+14$ & $1.12 \mathrm{E}+14$ & 1.27 \\
\hline & & $2.14 \mathrm{E}+15$ & $1.62 \mathrm{E}+15$ & 1.32 \\
\hline & \multirow{3}{*}{$\begin{array}{l}\text { Reflector Tally } \\
\text { Position } 1\end{array}$} & $5.30 \mathrm{E}+12$ & $4.48 \mathrm{E}+12$ & 1.18 \\
\hline & & $1.00 \mathrm{E}+15$ & $8.12 \mathrm{E}+14$ & 1.24 \\
\hline & & $1.17 \mathrm{E}+15$ & $9.35 \mathrm{E}+14$ & 1.25 \\
\hline \multirow{6}{*}{ Be-20 } & \multirow{3}{*}{ Center Flux Trap } & $3.12 \mathrm{E}+14$ & $2.31 \mathrm{E}+14$ & 1.35 \\
\hline & & $3.07 \mathrm{E}+14$ & $2.29 \mathrm{E}+14$ & 1.34 \\
\hline & & $2.07 \mathrm{E}+15$ & $1.53 \mathrm{E}+15$ & 1.35 \\
\hline & & $2.69 \mathrm{E}+14$ & $1.97 \mathrm{E}+14$ & 1.36 \\
\hline & Peripheral Flux & $2.64 \mathrm{E}+14$ & $1.97 \mathrm{E}+14$ & 1.34 \\
\hline & & $1.79 \mathrm{E}+15$ & $1.32 \mathrm{E}+15$ & 1.36 \\
\hline \multirow{6}{*}{$\begin{array}{c}\text { Initial } k_{e f f}=1.20621 \\
\mathrm{~B}_{1}=114 \text { days }\end{array}$} & \multirow{3}{*}{$\begin{array}{l}\text { Inner Fuel } \\
\text { Assembly }\end{array}$} & $4.91 \mathrm{E}+14$ & $3.68 \mathrm{E}+14$ & 1.33 \\
\hline & & $1.42 \mathrm{E}+14$ & $1.12 \mathrm{E}+14$ & 1.27 \\
\hline & & $2.10 \mathrm{E}+15$ & $1.58 \mathrm{E}+15$ & 1.33 \\
\hline & \multirow{3}{*}{$\begin{array}{l}\text { Reflector Tally } \\
\text { Position } 1\end{array}$} & $5.45 \mathrm{E}+12$ & $3.82 \mathrm{E}+12$ & 1.43 \\
\hline & & $9.51 \mathrm{E}+14$ & $7.70 \mathrm{E}+14$ & 1.24 \\
\hline & & $1.09 \mathrm{E}+15$ & $8.78 \mathrm{E}+14$ & 1.24 \\
\hline \multirow{12}{*}{$\mathrm{Be}-50$} & \multirow{3}{*}{ Center Flux Trap } & $2.92 \mathrm{E}+14$ & $2.17 \mathrm{E}+14$ & 1.35 \\
\hline & & $3.32 \mathrm{E}+14$ & $2.43 \mathrm{E}+14$ & 1.37 \\
\hline & & $2.05 \mathrm{E}+15$ & $1.51 \mathrm{E}+15$ & 1.36 \\
\hline & \multirow{3}{*}{$\begin{array}{l}\text { Peripheral Flux } \\
\text { Trap }\end{array}$} & $2.59 \mathrm{E}+14$ & $1.90 \mathrm{E}+14$ & 1.37 \\
\hline & & $2.83 \mathrm{E}+14$ & $2.14 \mathrm{E}+14$ & 1.32 \\
\hline & & $1.77 \mathrm{E}+15$ & $1.31 \mathrm{E}+15$ & 1.35 \\
\hline & \multirow{3}{*}{$\begin{array}{l}\text { Inner Fuel } \\
\text { Assembly }\end{array}$} & $4.86 \mathrm{E}+14$ & $3.62 \mathrm{E}+14$ & 1.34 \\
\hline & & $1.50 \mathrm{E}+14$ & $1.12 \mathrm{E}+14$ & 1.34 \\
\hline & & $2.11 \mathrm{E}+15$ & $1.55 \mathrm{E}+15$ & 1.36 \\
\hline & \multirow{3}{*}{$\begin{array}{l}\text { Reflector Tally } \\
\text { Position } 1\end{array}$} & $4.54 \mathrm{E}+12$ & $3.23 \mathrm{E}+12$ & 1.40 \\
\hline & & $9.38 \mathrm{E}+14$ & $7.48 \mathrm{E}+14$ & 1.25 \\
\hline & & $1.07 \mathrm{E}+15$ & $8.45 \mathrm{E}+14$ & 1.27 \\
\hline \multirow{12}{*}{$\begin{array}{l}\text { Initial } k_{e f f}=1.283 \\
\mathrm{~B}_{1}=165 \text { days }\end{array}$} & \multirow{3}{*}{ Center Flux Trap } & $2.68 \mathrm{E}+14$ & $1.94 \mathrm{E}+14$ & 1.38 \\
\hline & & $3.61 \mathrm{E}+14$ & $2.65 \mathrm{E}+14$ & 1.36 \\
\hline & & $2.00 \mathrm{E}+15$ & $1.44 \mathrm{E}+15$ & 1.39 \\
\hline & \multirow{3}{*}{$\begin{array}{c}\text { Peripheral Flux } \\
\text { Trap }\end{array}$} & $2.41 \mathrm{E}+14$ & $1.75 \mathrm{E}+14$ & 1.38 \\
\hline & & $3.24 \mathrm{E}+14$ & $2.39 \mathrm{E}+14$ & 1.35 \\
\hline & & $1.77 \mathrm{E}+15$ & $1.29 \mathrm{E}+15$ & 1.37 \\
\hline & \multirow{3}{*}{$\begin{array}{l}\text { Inner Fuel } \\
\text { Assembly }\end{array}$} & $4.74 \mathrm{E}+14$ & $3.46 \mathrm{E}+14$ & 1.37 \\
\hline & & $1.57 \mathrm{E}+14$ & $1.18 \mathrm{E}+14$ & 1.33 \\
\hline & & $2.05 \mathrm{E}+15$ & $1.51 \mathrm{E}+15$ & 1.36 \\
\hline & \multirow{3}{*}{$\begin{array}{l}\text { Reflector Tally } \\
\text { Position } 1\end{array}$} & $4.24 \mathrm{E}+12$ & $3.12 \mathrm{E}+12$ & 1.36 \\
\hline & & $9.41 \mathrm{E}+14$ & $7.56 \mathrm{E}+14$ & 1.24 \\
\hline & & $1.06 \mathrm{E}+15$ & $8.46 \mathrm{E}+14$ & 1.25 \\
\hline
\end{tabular}


Table 5-4. Fluence rates in pressure vessels for Annular and Cylindrical cores with various core rack contents.

\begin{tabular}{|c|c|c|}
\hline \multicolumn{2}{|c|}{ Core and Rack Content } & \multirow{2}{*}{ 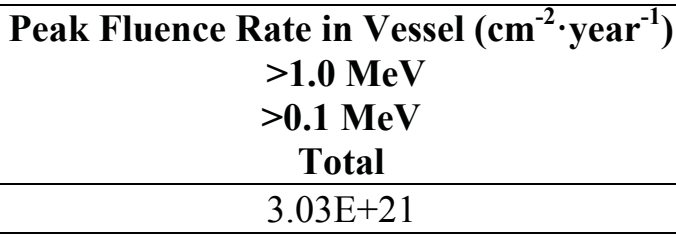 } \\
\hline \multirow{12}{*}{ Annular } & \multirow{3}{*}{$\mathrm{Be}-0$} & \\
\hline & & $8.52 \mathrm{E}+21$ \\
\hline & & $3.33 \mathrm{E}+22$ \\
\hline & \multirow{3}{*}{$\mathrm{Be}-20$} & $3.00 \mathrm{E}+21$ \\
\hline & & $9.09 \mathrm{E}+21$ \\
\hline & & $3.28 \mathrm{E}+22$ \\
\hline & \multirow{3}{*}{$\mathrm{Be}-50$} & $2.64 \mathrm{E}+21$ \\
\hline & & $6.80 \mathrm{E}+21$ \\
\hline & & $3.08 \mathrm{E}+22$ \\
\hline & \multirow{3}{*}{ Be-100 } & $1.88 \mathrm{E}+21$ \\
\hline & & $4.93 \mathrm{E}+21$ \\
\hline & & $2.70 \mathrm{E}+22$ \\
\hline \multirow{12}{*}{ Cylindrical } & \multirow{3}{*}{$\mathrm{Be}-0$} & $3.50 \mathrm{E}+21$ \\
\hline & & $9.78 \mathrm{E}+21$ \\
\hline & & $4.16 \mathrm{E}+22$ \\
\hline & \multirow{3}{*}{$\mathrm{Be}-20$} & $3.19 \mathrm{E}+21$ \\
\hline & & $8.83 \mathrm{E}+21$ \\
\hline & & $4.00 \mathrm{E}+22$ \\
\hline & \multirow{3}{*}{ Be-50 } & $2.83 \mathrm{E}+21$ \\
\hline & & $6.97 \mathrm{E}+21$ \\
\hline & & $4.26 \mathrm{E}+22$ \\
\hline & \multirow{3}{*}{ Be-100 } & $1.69 \mathrm{E}+21$ \\
\hline & & $5.87 \mathrm{E}+21$ \\
\hline & & $4.24 \mathrm{E}+22$ \\
\hline
\end{tabular}

\section{Alternative Fuel Options}

\section{$6.1 \quad \mathrm{U}_{3} \mathrm{Si}_{2}\left(6.0 \mathrm{~g} \mathrm{U} / \mathrm{cm}^{3}\right)$}

Monolithic U-10Mo fuel has not been fully qualified for use in a high heat flux test reactor at the time of this writing. Therefore, the reactor variants presented in this report were evaluated for their ability to use $\mathrm{U}_{3} \mathrm{Si}_{2}$ fuel, a fuel with lower uranium density than U-10Mo, but lower anticipated technical development risk as well. $\mathrm{U}_{3} \mathrm{Si}_{2}$ fuel can be fabricated with various volume fractions of aluminum. While $\mathrm{U}_{3} \mathrm{Si}_{2}$ fuel has been qualified with a uranium density of $4.8 \mathrm{~g} / \mathrm{cm}^{3}$, it is believed that a uranium density of $6 \mathrm{~g} / \mathrm{cm}^{3}\left(53 \%\right.$ by volume $\left.\mathrm{U}_{3} \mathrm{Si}_{2}\right)$ is feasible with relatively low development risk. This was therefore used for evaluating the possibility of using $\mathrm{U}_{3} \mathrm{Si}_{2}$ fuel in the reactor variants studied here. 
This analysis was performed by simply replacing the U-10Mo fuel meat with $\mathrm{U}_{3} \mathrm{Si}_{2}$ fuel having the same thickness in selected cases. The uranium enrichment was left at $19.7 \mathrm{w} / \mathrm{o}$ and the fuel temperature was not changed from the U-10Mo values used previously. For the Annular and Cylindrical cores, the various beryllium contents in the core rack presented in the previous section were also included in the analysis since they have a profound effect on reactivity. Table 6-1 shows initial $k_{\text {eff, }} B_{1}$, and neutron flux in various locations of the Annular core with $\mathrm{U}_{3} \mathrm{Si}_{2}$ fuel meat. With the aluminum core rack $(\mathrm{Be}-0)$, the reactor is barely critical at $\mathrm{BOL}$ and has essentially no burnup capability based on the $B_{I}$ value of 1 day. In contrast, the Be-100 core rack gives the Annular core much greater capability to accept the $\mathrm{U}_{3} \mathrm{Si}_{2}$ fuel with initial $k_{\text {eff }}$ of 1.27 and $B_{1}$ of 61 days. Flux values do not differ significantly between the $\mathrm{U}_{3} \mathrm{Si}_{2}$ cases and their respective U-10Mo cases.

Table 6-2 shows initial $k_{\text {eff, }} B_{1}$, and neutron flux in various locations of the Cylindrical core with $\mathrm{U}_{3} \mathrm{Si}_{2}$ fuel meat. With the aluminum core rack (Be-0), the reactor is has initial $k_{\text {eff }}$ of 1.11 and a $B_{1}$ value of 23 days. As with the Annular core the Be-100 core rack gives the Cylindrial core much greater capability to accept the $\mathrm{U}_{3} \mathrm{Si}_{2}$ fuel with initial $k_{\text {eff }}$ of 1.25 and $B_{1}$ of 61 days. Again, flux values do not differ significantly between the $\mathrm{U}_{3} \mathrm{Si}_{2}$ cases and their respective $\mathrm{U}$ 10Mo cases.

Table 6-3 and Table 6-4 show initial $k_{\text {eff, }}, B_{1}$, and neutron flux in various locations using $\mathrm{U}_{3} \mathrm{Si}_{2}$ fuel meat for the Square and PBT cores, respectively. The Square core using this fuel has an initial $k_{\text {eff }}$ value of 1.06 and a $B_{1}$ value of 5 days whereas the PBT core has initial $k_{\text {eff }}$ value of 1.26 and a $B_{1}$ value of 61 days. 
Table 6-1. Reactivity and flux performance of Annular concepts with $\mathrm{U}_{3} \mathrm{Si}_{2}$ fuel.

\begin{tabular}{|c|c|c|c|c|}
\hline \multirow{2}{*}{$\begin{array}{c}\text { Core Rack } \\
\text { Content } \\
\text { Initial } \boldsymbol{k}_{\text {eff }} \text { and } \mathbf{B}_{1}\end{array}$} & \multirow[t]{2}{*}{$\begin{array}{c}\text { Tally } \\
\text { Location }\end{array}$} & \multicolumn{3}{|c|}{$\begin{array}{c}\text { Neutron Flux }\left(\mathrm{n} \cdot \mathrm{cm}^{-2} \cdot \mathrm{s}^{-1}\right) \\
\text { Fast Flux }(>1 \mathrm{MeV}) \\
\text { Thermal Flux }(<0.625 \mathrm{eV}) \\
\text { Total Flux }\end{array}$} \\
\hline & & Peak & $\begin{array}{c}\text { Axially } \\
\text { Averaged }\end{array}$ & $\begin{array}{c}\text { Peak/ } \\
\text { Average }\end{array}$ \\
\hline \multirow{6}{*}{$\begin{array}{l}\text { (Baseline Case) } \\
\text { 93.7\% Aluminum } \\
\text { No Beryllium } \\
6.3 \% \mathrm{H}_{2} \mathrm{O}\end{array}$} & \multirow{3}{*}{$\begin{array}{c}\text { Center Flux } \\
\text { Trap }\end{array}$} & $3.30 \mathrm{E}+14$ & $2.48 \mathrm{E}+14$ & 1.33 \\
\hline & & $4.64 \mathrm{E}+14$ & $3.52 \mathrm{E}+14$ & 1.32 \\
\hline & & $2.30 \mathrm{E}+15$ & $1.73 \mathrm{E}+15$ & 1.33 \\
\hline & \multirow{3}{*}{$\begin{array}{c}\text { Peripheral Flux } \\
\text { Trap }\end{array}$} & $1.94 \mathrm{E}+14$ & $1.45 \mathrm{E}+14$ & 1.34 \\
\hline & & $2.94 \mathrm{E}+14$ & $2.21 \mathrm{E}+14$ & 1.33 \\
\hline & & $1.32 \mathrm{E}+15$ & $9.94 \mathrm{E}+14$ & 1.33 \\
\hline \multirow{3}{*}{$\begin{array}{c}\text { Initial } k_{\text {eff }}=1.04033 \\
\mathrm{~B}_{1}=1 \text { day }\end{array}$} & \multirow{3}{*}{$\begin{array}{c}\text { Reflector Tally } \\
\text { Position } 1\end{array}$} & $9.36 \mathrm{E}+12$ & $7.54 \mathrm{E}+12$ & 1.24 \\
\hline & & $6.93 \mathrm{E}+14$ & $5.65 \mathrm{E}+14$ & 1.23 \\
\hline & & $9.48 \mathrm{E}+14$ & $7.56 \mathrm{E}+14$ & 1.25 \\
\hline \multirow{5}{*}{$\begin{array}{c}73.7 \% \text { Aluminum } \\
20 \% \mathrm{Be} \\
6.3 \% \mathrm{H}_{2} \mathrm{O}\end{array}$} & \multirow{3}{*}{$\begin{array}{c}\text { Center Flux } \\
\text { Trap }\end{array}$} & $3.38 \mathrm{E}+14$ & $2.51 \mathrm{E}+14$ & 1.34 \\
\hline & & $4.87 \mathrm{E}+14$ & $3.68 \mathrm{E}+14$ & 1.32 \\
\hline & & $2.38 \mathrm{E}+15$ & $1.77 \mathrm{E}+15$ & 1.35 \\
\hline & \multirow{3}{*}{$\begin{array}{c}\text { Peripheral Flux } \\
\text { Trap }\end{array}$} & $1.85 \mathrm{E}+14$ & $1.39 \mathrm{E}+14$ & 1.34 \\
\hline & & $2.91 \mathrm{E}+14$ & $2.16 \mathrm{E}+14$ & 1.34 \\
\hline \multirow{4}{*}{$\begin{array}{c}\text { Initial } k_{e f f}=1.09238 \\
\mathrm{~B}_{1}=15 \text { days }\end{array}$} & & $1.29 \mathrm{E}+15$ & $9.60 \mathrm{E}+14$ & 1.34 \\
\hline & \multirow{3}{*}{$\begin{array}{l}\text { Reflector Tally } \\
\text { Position } 1\end{array}$} & $7.86 \mathrm{E}+12$ & $5.84 \mathrm{E}+12$ & 1.35 \\
\hline & & $6.87 \mathrm{E}+14$ & $5.43 \mathrm{E}+14$ & 1.27 \\
\hline & & $8.88 \mathrm{E}+14$ & $6.93 \mathrm{E}+14$ & 1.28 \\
\hline \multirow{5}{*}{$\begin{array}{c}\text { 43.7\% Aluminum } \\
50 \% \mathrm{Be} \\
6.3 \% \mathrm{H}_{2} \mathrm{O}\end{array}$} & \multirow{3}{*}{$\begin{array}{c}\text { Center Flux } \\
\text { Trap }\end{array}$} & $3.24 \mathrm{E}+14$ & $2.37 \mathrm{E}+14$ & 1.37 \\
\hline & & $5.04 \mathrm{E}+14$ & $3.71 \mathrm{E}+14$ & 1.36 \\
\hline & & $2.35 \mathrm{E}+15$ & $1.71 \mathrm{E}+15$ & 1.37 \\
\hline & \multirow{3}{*}{$\begin{array}{c}\text { Peripheral Flux } \\
\text { Trap }\end{array}$} & $1.83 \mathrm{E}+14$ & $1.34 \mathrm{E}+14$ & 1.36 \\
\hline & & $2.97 \mathrm{E}+14$ & $2.16 \mathrm{E}+14$ & 1.37 \\
\hline \multirow{4}{*}{$\begin{array}{c}\text { Initial } k_{e f f}=1.15601 \\
\mathrm{~B}_{1}=34 \text { days }\end{array}$} & & $1.28 \mathrm{E}+15$ & $9.39 \mathrm{E}+14$ & 1.37 \\
\hline & \multirow{3}{*}{$\begin{array}{l}\text { Reflector Tally } \\
\text { Position } 1\end{array}$} & $6.33 \mathrm{E}+12$ & $4.72 \mathrm{E}+12$ & 1.34 \\
\hline & & $6.61 \mathrm{E}+14$ & $5.25 \mathrm{E}+14$ & 1.26 \\
\hline & & $8.23 \mathrm{E}+14$ & $6.45 \mathrm{E}+14$ & 1.28 \\
\hline \multirow{9}{*}{$\begin{array}{l}\text { No Aluminum } \\
93.7 \% \mathrm{Be} \\
6.3 \% \mathrm{H}_{2} \mathrm{O}\end{array}$} & \multirow{3}{*}{$\begin{array}{c}\text { Center Flux } \\
\text { Trap }\end{array}$} & $2.86 \mathrm{E}+14$ & $2.08 \mathrm{E}+14$ & 1.38 \\
\hline & & $4.99 \mathrm{E}+14$ & $3.61 \mathrm{E}+14$ & 1.38 \\
\hline & & $2.13 \mathrm{E}+15$ & $1.54 \mathrm{E}+15$ & 1.38 \\
\hline & \multirow{3}{*}{$\begin{array}{c}\text { Peripheral Flux } \\
\text { Trap }\end{array}$} & $1.89 \mathrm{E}+14$ & $1.37 \mathrm{E}+14$ & 1.38 \\
\hline & & $3.07 \mathrm{E}+14$ & $2.23 \mathrm{E}+14$ & 1.38 \\
\hline & & $1.31 \mathrm{E}+15$ & $9.49 \mathrm{E}+14$ & 1.38 \\
\hline & \multirow{3}{*}{$\begin{array}{c}\text { Reflector Tally } \\
\text { Position } 1\end{array}$} & $5.01 \mathrm{E}+12$ & $3.78 \mathrm{E}+12$ & 1.33 \\
\hline & & $7.22 \mathrm{E}+14$ & $5.51 \mathrm{E}+14$ & 1.31 \\
\hline & & $8.47 \mathrm{E}+14$ & $6.43 \mathrm{E}+14$ & 1.32 \\
\hline
\end{tabular}


Table 6-2. Reactivity and flux performance of Cylindrical concepts with $\mathrm{U}_{3} \mathrm{Si}_{2}$ fuel.

\begin{tabular}{|c|c|c|c|c|}
\hline \multirow[t]{2}{*}{$\begin{array}{l}\text { Core Rack Content } \\
\text { Initial } k_{\text {eff }} \text { and } B_{1}\end{array}$} & \multirow[t]{2}{*}{$\begin{array}{c}\text { Tally } \\
\text { Location }\end{array}$} & \multicolumn{3}{|c|}{ 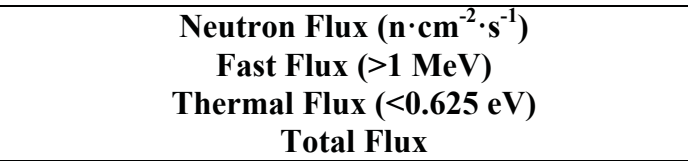 } \\
\hline & & Peak & $\begin{array}{c}\text { Axially } \\
\text { Averaged }\end{array}$ & $\begin{array}{c}\text { Peak/ } \\
\text { Average }\end{array}$ \\
\hline \multirow{7}{*}{$\begin{array}{l}\text { (Baseline Case) } \\
\text { 93.7\% Aluminum } \\
\text { No Beryllium } \\
6.3 \% \mathrm{H}_{2} \mathrm{O}\end{array}$} & \multirow{3}{*}{ Center Flux Trap } & $3.39 \mathrm{E}+14$ & $2.52 \mathrm{E}+14$ & 1.35 \\
\hline & & $3.57 \mathrm{E}+14$ & $2.70 \mathrm{E}+14$ & 1.32 \\
\hline & & $2.23 \mathrm{E}+15$ & $1.65 \mathrm{E}+15$ & 1.35 \\
\hline & \multirow{3}{*}{$\begin{array}{c}\text { Peripheral Flux } \\
\text { Trap }\end{array}$} & $2.95 \mathrm{E}+14$ & $2.19 \mathrm{E}+14$ & 1.35 \\
\hline & & $3.09 \mathrm{E}+14$ & $2.37 \mathrm{E}+14$ & 1.30 \\
\hline & & $1.91 \mathrm{E}+15$ & $1.44 \mathrm{E}+15$ & 1.33 \\
\hline & \multirow{3}{*}{$\begin{array}{l}\text { Inner Fuel } \\
\text { Assembly }\end{array}$} & $5.29 \mathrm{E}+14$ & $4.04 \mathrm{E}+14$ & 1.31 \\
\hline \multirow{5}{*}{$\begin{array}{c}\text { Initial } k_{e f f}=1.11269 \\
\mathrm{~B}_{1}=23 \text { days }\end{array}$} & & $3.52 \mathrm{E}+14$ & $2.64 \mathrm{E}+14$ & 1.33 \\
\hline & & $2.45 \mathrm{E}+15$ & $1.85 \mathrm{E}+15$ & 1.33 \\
\hline & \multirow{3}{*}{$\begin{array}{l}\text { Reflector Tally } \\
\text { Position } 1\end{array}$} & $5.89 \mathrm{E}+12$ & $4.77 \mathrm{E}+12$ & 1.23 \\
\hline & & $1.06 \mathrm{E}+15$ & $8.76 \mathrm{E}+14$ & 1.21 \\
\hline & & $1.22 \mathrm{E}+15$ & $1.00 \mathrm{E}+15$ & 1.22 \\
\hline \multirow{7}{*}{$\begin{array}{c}73.7 \% \text { Aluminum } \\
20 \% \mathrm{Be} \\
6.3 \% \mathrm{H}_{2} \mathrm{O}\end{array}$} & \multirow{3}{*}{ Center Flux Trap } & $3.28 \mathrm{E}+14$ & $2.42 \mathrm{E}+14$ & 1.36 \\
\hline & & $3.88 \mathrm{E}+14$ & $2.92 \mathrm{E}+14$ & 1.33 \\
\hline & & $2.22 \mathrm{E}+15$ & $1.65 \mathrm{E}+15$ & 1.35 \\
\hline & \multirow{3}{*}{$\begin{array}{c}\text { Peripheral Flux } \\
\text { Trap }\end{array}$} & $2.85 \mathrm{E}+14$ & $2.10 \mathrm{E}+14$ & 1.36 \\
\hline & & $3.41 \mathrm{E}+14$ & $2.53 \mathrm{E}+14$ & 1.35 \\
\hline & & $1.93 \mathrm{E}+15$ & $1.43 \mathrm{E}+15$ & 1.36 \\
\hline & \multirow{3}{*}{$\begin{array}{l}\text { Inner Fuel } \\
\text { Assembly }\end{array}$} & $5.36 \mathrm{E}+14$ & $4.02 \mathrm{E}+14$ & 1.33 \\
\hline \multirow{5}{*}{$\begin{array}{c}\text { Initial } k_{\text {eff }}=1.14250 \\
\mathrm{~B}_{1}=33 \text { days }\end{array}$} & & $3.62 \mathrm{E}+14$ & $2.72 \mathrm{E}+14$ & 1.33 \\
\hline & & $2.51 \mathrm{E}+15$ & $1.86 \mathrm{E}+15$ & 1.35 \\
\hline & \multirow{3}{*}{$\begin{array}{l}\text { Reflector Tally } \\
\text { Position } 1\end{array}$} & $5.58 \mathrm{E}+12$ & $4.20 \mathrm{E}+12$ & 1.33 \\
\hline & & $1.06 \mathrm{E}+15$ & $8.49 \mathrm{E}+14$ & 1.25 \\
\hline & & $1.21 \mathrm{E}+15$ & $9.62 \mathrm{E}+14$ & 1.25 \\
\hline \multirow{12}{*}{$\begin{array}{c}43.7 \% \text { Aluminum } \\
50 \% \mathrm{Be} \\
6.3 \% \mathrm{H}_{2} \mathrm{O} \\
\text { Initial } k_{\text {eff }}=1.18330 \\
\mathrm{~B}_{1}=45 \text { days }\end{array}$} & \multirow{3}{*}{ Center Flux Trap } & $3.09 \mathrm{E}+14$ & $2.26 \mathrm{E}+14$ & 1.36 \\
\hline & & $4.08 \mathrm{E}+14$ & $3.05 \mathrm{E}+14$ & 1.34 \\
\hline & & $2.19 \mathrm{E}+15$ & $1.61 \mathrm{E}+15$ & 1.35 \\
\hline & \multirow{3}{*}{$\begin{array}{c}\text { Peripheral Flux } \\
\text { Trap }\end{array}$} & $2.68 \mathrm{E}+14$ & $1.95 \mathrm{E}+14$ & 1.37 \\
\hline & & $3.60 \mathrm{E}+14$ & $2.69 \mathrm{E}+14$ & 1.34 \\
\hline & & $1.91 \mathrm{E}+15$ & $1.41 \mathrm{E}+15$ & 1.36 \\
\hline & \multirow{3}{*}{$\begin{array}{l}\text { Inner Fuel } \\
\text { Assembly }\end{array}$} & $5.34 \mathrm{E}+14$ & $3.87 \mathrm{E}+14$ & 1.38 \\
\hline & & $3.81 \mathrm{E}+14$ & $2.79 \mathrm{E}+14$ & 1.36 \\
\hline & & $2.49 \mathrm{E}+15$ & $1.81 \mathrm{E}+15$ & 1.37 \\
\hline & \multirow{3}{*}{$\begin{array}{l}\text { Reflector Tally } \\
\text { Position } 1\end{array}$} & $4.64 \mathrm{E}+12$ & $3.40 \mathrm{E}+12$ & 1.36 \\
\hline & & $1.06 \mathrm{E}+15$ & $8.38 \mathrm{E}+14$ & 1.26 \\
\hline & & $1.19 \mathrm{E}+15$ & $9.38 \mathrm{E}+14$ & 1.26 \\
\hline \multirow{12}{*}{$\begin{array}{l}\text { No Aluminum } \\
93.7 \% \mathrm{Be} \\
6.3 \% \mathrm{H}_{2} \mathrm{O}\end{array}$} & \multirow{3}{*}{ Center Flux Trap } & $2.78 \mathrm{E}+14$ & $2.02 \mathrm{E}+14$ & 1.38 \\
\hline & & $4.53 \mathrm{E}+14$ & $3.32 \mathrm{E}+14$ & 1.36 \\
\hline & & $2.10 \mathrm{E}+15$ & $1.54 \mathrm{E}+15$ & 1.37 \\
\hline & & $2.52 \mathrm{E}+14$ & $1.81 \mathrm{E}+14$ & 1.39 \\
\hline & Peripheral Fiux & $4.04 \mathrm{E}+14$ & $2.95 \mathrm{E}+14$ & 1.37 \\
\hline & & $1.91 \mathrm{E}+15$ & $1.38 \mathrm{E}+15$ & 1.38 \\
\hline & & $4.91 \mathrm{E}+14$ & $3.61 \mathrm{E}+14$ & 1.36 \\
\hline & $\begin{array}{l}\text { Inner Fuel } \\
\text { Assembly }\end{array}$ & $4.08 \mathrm{E}+14$ & $3.06 \mathrm{E}+14$ & 1.34 \\
\hline & & $2.43 \mathrm{E}+15$ & $1.78 \mathrm{E}+15$ & 1.36 \\
\hline & & $4.44 \mathrm{E}+12$ & $3.12 \mathrm{E}+12$ & 1.42 \\
\hline & Renlector 1 anty & $1.05 \mathrm{E}+15$ & $8.36 \mathrm{E}+14$ & 1.25 \\
\hline & & $1.17 \mathrm{E}+15$ & $9.25 \mathrm{E}+14$ & 1.27 \\
\hline
\end{tabular}


Table 6-3. Reactivity and flux performance of baseline Square concept with $\mathrm{U}_{3} \mathrm{Si}_{2}$ fuel. ${ }^{\mathrm{a}}$

\begin{tabular}{|c|c|c|c|}
\hline \multirow[t]{2}{*}{ Tally Location } & \multicolumn{3}{|c|}{ 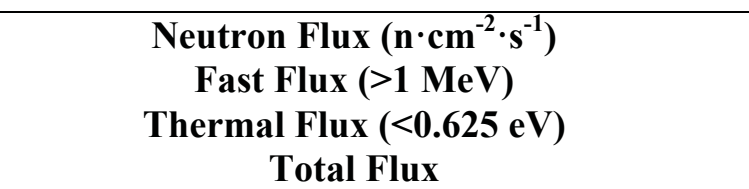 } \\
\hline & Peak & $\begin{array}{c}\text { Axially } \\
\text { Averaged }\end{array}$ & $\begin{array}{c}\text { Peak/ } \\
\text { Average }\end{array}$ \\
\hline \multirow{3}{*}{ Center Flux Trap } & $2.87 \mathrm{E}+14$ & $2.19 \mathrm{E}+14$ & 1.31 \\
\hline & $3.62 \mathrm{E}+14$ & $2.78 \mathrm{E}+14$ & 1.30 \\
\hline & $2.19 \mathrm{E}+15$ & $1.65 \mathrm{E}+15$ & 1.33 \\
\hline \multirow{3}{*}{ NW Flux Trap } & $2.88 \mathrm{E}+14$ & $2.18 \mathrm{E}+14$ & 1.32 \\
\hline & $3.11 \mathrm{E}+14$ & $2.37 \mathrm{E}+14$ & 1.31 \\
\hline & $1.95 \mathrm{E}+15$ & $1.48 \mathrm{E}+15$ & 1.32 \\
\hline \multirow{3}{*}{ Reflector Tally Position 1} & $8.44 \mathrm{E}+12$ & $5.84 \mathrm{E}+12$ & 1.45 \\
\hline & $1.05 \mathrm{E}+15$ & $8.30 \mathrm{E}+14$ & 1.26 \\
\hline & $1.27 \mathrm{E}+15$ & $9.99 \mathrm{E}+14$ & 1.27 \\
\hline
\end{tabular}

Table 6-4. Reactivity and flux performance of baseline PBT concept with $\mathrm{U}_{3} \mathrm{Si}_{2}$ fuel. ${ }^{\mathrm{b}}$

\begin{tabular}{|c|c|c|c|}
\hline \multirow[t]{2}{*}{ Tally Location } & \multicolumn{3}{|c|}{$\begin{array}{c}\text { Neutron Flux }\left(\mathrm{n} \cdot \mathrm{cm}^{-2} \cdot \mathrm{s}^{-1}\right) \\
\text { Fast Flux }(>1 \mathrm{MeV}) \\
\text { Thermal Flux }(<0.625 \mathrm{eV}) \\
\text { Total Flux } \\
\end{array}$} \\
\hline & Peak & $\begin{array}{c}\text { Axially } \\
\text { Averaged }\end{array}$ & $\begin{array}{c}\text { Peak/ } \\
\text { Average }\end{array}$ \\
\hline \multirow{3}{*}{ Center Flux Trap } & $2.43 \mathrm{E}+14$ & $1.81 \mathrm{E}+14$ & 1.34 \\
\hline & $4.09 \mathrm{E}+14$ & $3.13 \mathrm{E}+14$ & 1.31 \\
\hline & $1.73 \mathrm{E}+15$ & $1.31 \mathrm{E}+15$ & 1.32 \\
\hline \multirow{3}{*}{ Peripheral Flux Trap } & $1.76 \mathrm{E}+14$ & $1.33 \mathrm{E}+14$ & 1.32 \\
\hline & $2.88 \mathrm{E}+14$ & $2.18 \mathrm{E}+14$ & 1.32 \\
\hline & $1.20 \mathrm{E}+15$ & $9.09 \mathrm{E}+14$ & 1.32 \\
\hline \multirow{3}{*}{ Reflector Tally Position 1} & $4.04 \mathrm{E}+12$ & $3.52 \mathrm{E}+12$ & 1.15 \\
\hline & $1.33 \mathrm{E}+15$ & $1.09 \mathrm{E}+15$ & 1.23 \\
\hline & $1.45 \mathrm{E}+15$ & $1.18 \mathrm{E}+15$ & 1.23 \\
\hline
\end{tabular}

${ }^{\text {a }}$ Initial $k_{\text {eff }}=1.06337, B_{I}=5$ days

${ }^{\mathrm{b}}$ Initial $k_{\text {eff }}=1.25558, B_{I}=61$ days 


\section{Comparisons to Serpent Model of ATR}

\subsection{Description of Serpent ATR Model}

To evaluate whether the proposed MATRIX designs can meet specified requirements, particularly those adopted from the NR requirements (Section 1.2), comparisons to a detailed ATR model were performed. A Serpent 2 model of ATR was adopted from M. DeHart (INL). This model was based on the 1994 Core Internals Changeout (94-CIC configuration). A core loaded in conjunction with the 94-CIC was comprised entirely of fresh HEU Mark VII fuel. This core was never operated at full power, but used as a low-power critical configuration to validate computational models. This core configuration was later described in detail and archived in the International Handbook of Evaluated Reactor Physics Benchmark Experiments [17]. A schematic of the core configuration is given in Figure 7-1.

The general layout of the ATR core is a serpentine arrangement of arcuate fuel assemblies containing plates of HEU fuel. The fuel meat consists of $93 \mathrm{w} / \mathrm{o}$ enriched uranium aluminide powder $\left(\mathrm{UAl}_{\mathrm{x}}\right)$ dispersed in aluminum. The cladding is Al-6061 and the fuel meat, cladding, and coolant channel thicknesses are the same as those of the MATRIX baseline configurations. Reactivity is held down by 1) neck shim rods located in the neck shim rod housing 2) rotating Outer Shim Control Cylinders (OSCCs) having a surface partially covered with hafnium absorber and 3) boron carbide burnable poison mixed into the fuel meat of the inner and outer four plates of each fuel assembly.

The 94-CIC configuration specifies that the test region of the NW IPT be filled with Al-6061. The W, SW, and SE IPTs were filled with coolant $\mathrm{H}_{2} \mathrm{O}$. Deviations made from the exact 94-CIC specifications for the purposes of this study are described below. For a full description of the 94CIC test configuration, see Reference [17].

Comparisons to this ATR configuration are useful in the MATRIX project for a number of reasons. First, some of the requirements enumerated in Section 1.2 are borrowed from the LEU conversion project of ATR. Among these, some require a minimum change of a parameter from ATR. In evaluating whether or not these requirements are met, one must have analogous ATR calculations for comparison. More generally, the MATRIX will be expected to perform as well or better than ATR by most metrics. Therefore, comparing to an ATR model allows one to predict whether or not an improvement over ATR can be expected without actually knowing the real performance with a high degree of certainty.

In the MATRIX studies presented thus far, the actual reactor models are very simplified. For example; omission of burnable poisons and control rods, fully fresh cores depleted in a single pass, and uniform temperatures. While these assumptions are necessary to accommodate the large option space in early studies, they do make comparisons to real data from the operating ATR less meaningful.

To accommodate this, the 94-CIC model from DeHart was modified in the following ways to be more analogous to the MATRIX calculations performed here:

- In the ATR's Mark VII HEU fuel, the inner and outer four fuel plates have boron carbide mixed into the fuel meat. The boron carbide was removed from the fuel meat of these 
plates leaving the uranium nuclides as they were. This gives a model with the same amount of HM as the actual ATR core but with no burnable poison.

- All neck shim rods were withdrawn.

- The hafnium absorber material on the surface of the OSCCs was replaced with beryllium.

- The SW flux trap was filled with Al-6061, leaving W and SE filled with $\mathrm{H}_{2} \mathrm{O}$ as specified for the benchmark.

- Reactor power was set to $250 \mathrm{MW}_{\text {th }}$ rather than the zero power of the 94-CIC critical or the $\sim 120 \mathrm{MW}_{\text {th }}$ at which ATR is typically operated.

This provides a "flat" core (meaning not purposefully tilted as is common in operation of ATR) with all fresh unpoisoned HEU fuel. Depletion was performed on this model without changing the control shim position or OSCC rotation and neutron flux tallies were calculated at each burnup step. This methodology provides an analogous ATR case to which one may compare the MATRIX cases. This model was depleted with flux tallies in four different positions; the NW flux trap, the SW flux trap, the SE flux trap, center capsule location \#3, and the A-1 hole. 


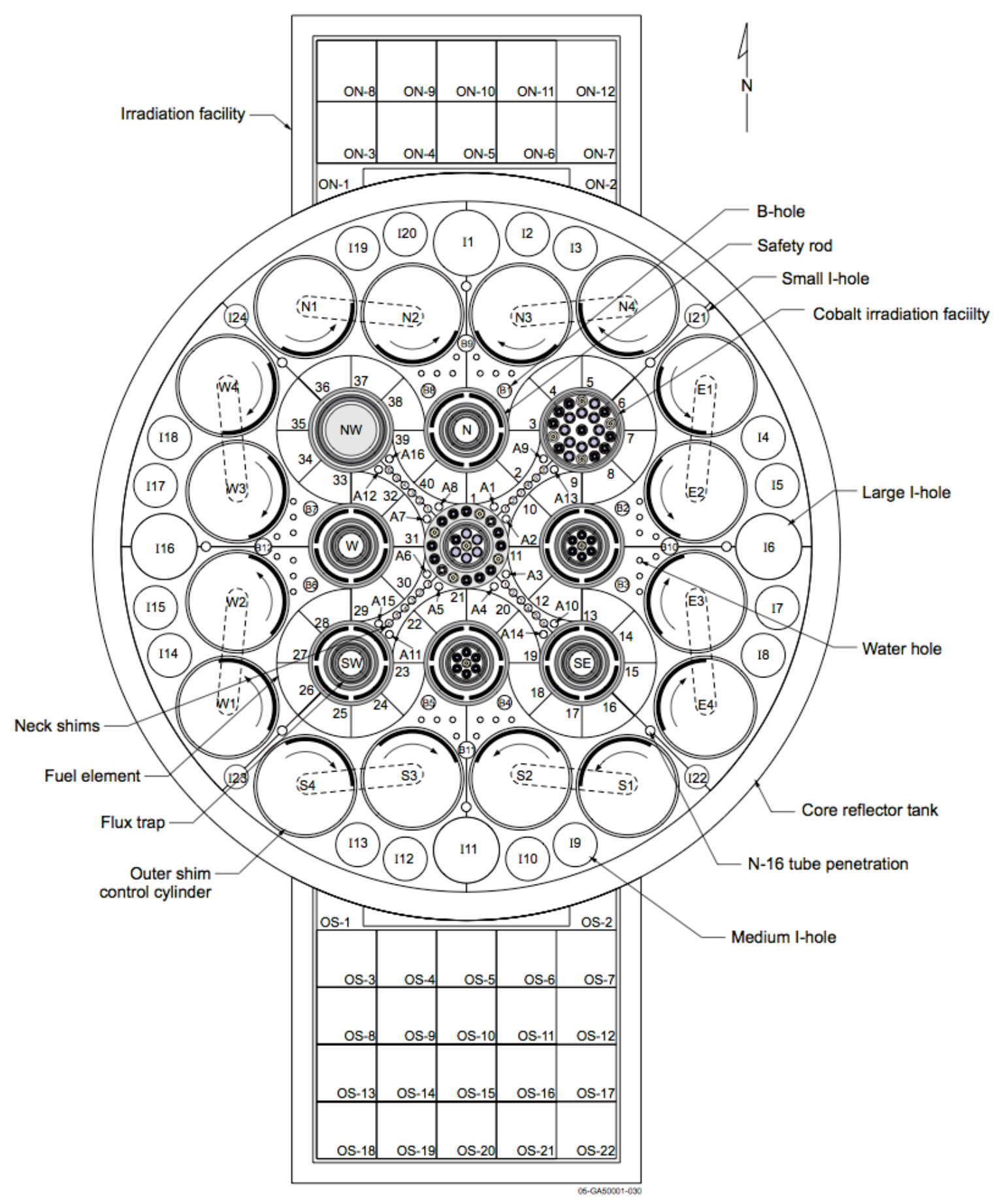

Figure 7-1. Cross-sectional view of the ATR core in the 94-CIC configuration. [17] 


\subsection{Initial Results of ATR Depletion Analysis}

As was done for the baseline MATRIX case studies, $B_{1}$ was calculated by linear interpolation and the detector tallies were taken from the burnup step closest to $k_{\text {eff }}=1$. The initial $k_{\text {eff }}$ for this core was calculated to be 1.222 and the $B_{1}$ value was 56 days. Table $7-1$ shows flux values in the locations described above. As with the MATRIX studies, the tallies spanned the entire axial length of the active core and were divided into 10 axial segments. Peak/average ratios are given for each tally.

As seen in the table, the aluminum-filled NW and SW flux traps have fast flux values of approximately $2.9 \times 10^{14}$ and $3.5 \times 10^{14} \mathrm{n} \cdot \mathrm{cm}^{-2} \cdot \mathrm{s}^{-1}$, respectively, and total fluxes of $1.9 \times 10^{15}$ and $2.3 \times 10^{15} \mathrm{n} \cdot \mathrm{cm}^{-2} \cdot \mathrm{s}^{-1}$, respectively. In the water-filled SE flux trap, the thermal and total flux are $1.2 \times 10^{15}$ and $2.4 \times 10^{15} \mathrm{n}^{\cdot} \mathrm{cm}^{-2} \cdot \mathrm{s}^{-1}$, respectively. The highest fast flux of the locations sampled here was located in the A-1 hole filled with an aluminum flow restrictor. This location had a fast neutron flux of $4.4 \times 10^{14} \mathrm{n} \cdot \mathrm{cm}^{-2} \cdot \mathrm{s}^{-1}$.

Table 7-1. Flux values from simplified ATR model depletion calculation. ${ }^{\mathrm{a}}$

\begin{tabular}{|c|c|c|c|}
\hline \multirow{2}{*}{$\begin{array}{c}\text { Tally Location } \\
\text { and } \\
\text { Location Content }\end{array}$} & \multicolumn{3}{|c|}{$\begin{array}{c}\text { Neutron Flux }\left(\mathrm{n} \cdot \mathrm{cm}^{-2} \cdot \mathrm{s}^{-1}\right) \\
\text { Fast Flux }(>1 \mathrm{MeV}) \\
\text { Thermal Flux }(<0.625 \mathrm{eV}) \\
\text { Total Flux }\end{array}$} \\
\hline & Peak & $\begin{array}{c}\text { Axially } \\
\text { Averaged }\end{array}$ & $\begin{array}{c}\text { Peak/ } \\
\text { Average }\end{array}$ \\
\hline \multirow{3}{*}{$\begin{array}{l}\text { NW Flux Trap } \\
\text { Al-6061 filled }\end{array}$} & $2.89 \mathrm{E}+14$ & $2.08 \mathrm{E}+14$ & 1.39 \\
\hline & $4.47 \mathrm{E}+14$ & $3.20 \mathrm{E}+14$ & 1.40 \\
\hline & $1.87 \mathrm{E}+15$ & $1.33 \mathrm{E}+15$ & 1.40 \\
\hline \multirow{3}{*}{$\begin{array}{l}\text { SW Flux Trap } \\
\text { Al-6061 filled }\end{array}$} & $3.48 \mathrm{E}+14$ & $2.48 \mathrm{E}+14$ & 1.40 \\
\hline & $5.30 \mathrm{E}+14$ & $3.68 \mathrm{E}+14$ & 1.44 \\
\hline & $2.28 \mathrm{E}+15$ & $1.60 \mathrm{E}+15$ & 1.42 \\
\hline \multirow{3}{*}{$\begin{array}{l}\text { SE Flux Trap } \\
\mathrm{H}_{2} \mathrm{O} \text { filled }\end{array}$} & $2.29 \mathrm{E}+14$ & $1.60 \mathrm{E}+14$ & 1.43 \\
\hline & $1.22 \mathrm{E}+15$ & $8.65 \mathrm{E}+14$ & 1.41 \\
\hline & $2.38 \mathrm{E}+15$ & $1.68 \mathrm{E}+15$ & 1.42 \\
\hline \multirow{3}{*}{$\begin{array}{c}\text { Center Capsule Location \#3 } \\
\text { Al-6061 filled }\end{array}$} & $2.99 \mathrm{E}+14$ & $2.10 \mathrm{E}+14$ & 1.42 \\
\hline & $1.26 \mathrm{E}+15$ & $9.24 \mathrm{E}+14$ & 1.37 \\
\hline & $2.67 \mathrm{E}+15$ & $1.95 \mathrm{E}+15$ & 1.37 \\
\hline \multirow{3}{*}{$\begin{array}{c}\text { A-1 Hole } \\
\text { Al-6061 flow restrictor }\end{array}$} & $4.44 \mathrm{E}+14$ & $3.20 \mathrm{E}+14$ & 1.38 \\
\hline & $7.24 \mathrm{E}+14$ & $5.23 \mathrm{E}+14$ & 1.38 \\
\hline & $2.53 \mathrm{E}+15$ & $1.81 \mathrm{E}+15$ & 1.40 \\
\hline
\end{tabular}

${ }^{\mathrm{a}} B_{1}=56$ days, Initial $k_{\text {eff }}=1.22208$

${ }^{\mathrm{b}}$ Denotes different filler than that specified in the 94-CIC benchmark. 


\subsection{Comparison of Fast-to-Thermal Flux Ratios}

One of the NR requirements for a converted ATR is a fast-to-thermal neutron flux ratio within $5 \%$ of current values in a pressurized-water loop test. The same requirement could be directly applied to the MATRIX designs. In order to compare these ratios between ATR and the various MATRIX concepts, selected flux tally data already reported in this document was organized into a single table of fast-to-thermal flux ratios. The result of this is shown in Table $7-2$.

In the simplified ATR case described in Section 7.1, the fast-to-thermal flux ratios range from 2.5-3.3 in the aluminum-filled locations to just below 1 in the water-filled SE flux trap. As for the MATRIX cases, a number of test locations in various baseline and variant reactor designs are evaluated, not all of which will be discussed in the text. Several of these, however, warrant comment.

In the Cylindrical baseline reactor with the aluminum core rack, the center and peripheral IPT locations have fast-to-thermal flux ratios of approximately 6 while the location at the center of a centrally located fuel assembly has a ratio of 14 . These are all for aluminum-filled test locations. The very high ratio is a result of the use of the low-moderating aluminum core rack and the resulting hard spectrum this facilitates. When these same locations are filled with water, the reduction in fast-to-thermal ratio is substantial. The IPTs and the assembly location then have ratios of 0.6 and 2.3, respectively. This substantial change in the case of the IPTs is partially a result of the rather large size of all of the IPTs in the Cylindrical core model $(9.3 \mathrm{~cm}$ dia.). These are approximately the size of the NW IPT in ATR $(9.0 \mathrm{~cm}$ dia.), significantly larger than the SE and SW IPTs in ATR (4.4 cm dia.). The large diameter of these locations provides a large amount of thermalization when totally water-filled. If the Cylindrical core rack is changed to the $100 \%$ beryllium case, the aluminum-filled test locations see a significant reduction in fastto-thermal flux ratios. For these test locations, when water-filled, the rack material has little impact. This is because in the IPTs, there is present enough water to provide rather complete thermalization, and in the location inside the fuel assembly, the spectrum is decoupled from the environment outside the fuel assembly.

Due to the similarity in design between the Square and Cylindrical cores, the fast-to-thermal ratios between their IPT locations are quite similar, as can be seen in Table 7-2.

With regard to the Annular and PBT designs, two main trends are notable. First, the thermalization is quite strong in the very large center IPTs $(12.7 \mathrm{~cm}$ dia.) in both cases when water-filled. Also, the difference in rack content between aluminum and beryllium makes little difference in either core design. This is a result of the decoupling between outside and inside the fuel assembly modules, much as in the case of the inter-assembly locations of the Cylindrical core. 
Table 7-2. Fast-to-thermal flux ratios in selected MATRIX cases and ATR model.

\begin{tabular}{|c|c|c|c|}
\hline Reactor & Location & Content & $\begin{array}{l}\text { Fast-to-Thermal Flux } \\
\text { Ratio at Axial Center } \\
\text { (Cutoff at } 0.625 \mathrm{eV} \text { ) }\end{array}$ \\
\hline \multirow{5}{*}{$\begin{array}{c}\text { ATR } \\
\text { Simplified case } \\
\text { described in } \\
\text { Section } 7.1\end{array}$} & NW Flux Trap & Al-6061 filled & 3.18 \\
\hline & SW Flux Trap & Al-6061 filled & 3.30 \\
\hline & SE Flux Trap & $\mathrm{H}_{2} \mathrm{O}$ filled & 0.95 \\
\hline & Center Capsule Loc. \#3 & Al-6061 filled & 1.12 \\
\hline & A-1 Hole & Al-6061 filled & 2.49 \\
\hline \multirow{6}{*}{$\begin{array}{c}\text { Cylindrical } \\
\text { MATRIX } \\
\text { Baseline Al Rack }\end{array}$} & Center IPT & Al-6061 filled & 6.47 \\
\hline & Peripheral IPT & Al-6061 filled & 6.44 \\
\hline & Inside Fuel Assy & Al-6061 filled & 14.07 \\
\hline & Center IPT & $\mathrm{H}_{2} \mathrm{O}$ filled & 0.58 \\
\hline & Peripheral IPT & $\mathrm{H}_{2} \mathrm{O}$ filled & 0.56 \\
\hline & Inside Fuel Assy & $\mathrm{H}_{2} \mathrm{O}$ filled & 2.31 \\
\hline \multirow{6}{*}{$\begin{array}{c}\text { Cylindrical } \\
\text { MATRIX } \\
\text { 100\% Be Rack }\end{array}$} & Center IPT & Al-6061 filled & 4.54 \\
\hline & Peripheral IPT & Al-6061 filled & 4.46 \\
\hline & Inside Fuel Assy & Al-6061 filled & 12.06 \\
\hline & Center IPT & $\mathrm{H}_{2} \mathrm{O}$ filled & 0.52 \\
\hline & Peripheral IPT & $\mathrm{H}_{2} \mathrm{O}$ filled & 0.53 \\
\hline & Inside Fuel Assy & $\mathrm{H}_{2} \mathrm{O}$ filled & 2.09 \\
\hline \multirow{2}{*}{$\begin{array}{c}\text { Square } \\
\text { MATRIX } \\
\text { Baseline }\end{array}$} & Center IPT & Al-6061 filled & 6.04 \\
\hline & NW IPT & Al-6061 filled & 6.59 \\
\hline \multirow{4}{*}{$\begin{array}{c}\text { Annular } \\
\text { MATRIX } \\
\text { Baseline Al Rack }\end{array}$} & Center IPT & Al-6061 filled & 4.80 \\
\hline & Peripheral IPT & Al-6061 filled & 4.06 \\
\hline & Center IPT & $\mathrm{H}_{2} \mathrm{O}$ filled & 0.34 \\
\hline & Peripheral IPT & $\mathrm{H}_{2} \mathrm{O}$ filled & 0.75 \\
\hline \multirow{4}{*}{$\begin{array}{c}\begin{array}{c}\text { Annular } \\
\text { MATRIX } \\
100 \% \text { Be Rack }\end{array}\end{array}$} & Center IPT & Al-6061 filled & 4.15 \\
\hline & Peripheral IPT & Al-6061 filled & 3.76 \\
\hline & Center IPT & $\mathrm{H}_{2} \mathrm{O}$ filled & 0.34 \\
\hline & Peripheral IPT & $\mathrm{H}_{2} \mathrm{O}$ filled & 0.73 \\
\hline \multirow{2}{*}{$\begin{array}{c}\text { PBT } \\
\text { MATRIX } \\
\text { Baseline }\end{array}$} & Center IPT & Al-6061 filled & 4.13 \\
\hline & Peripheral IPT & Al-6061 filled & 3.70 \\
\hline
\end{tabular}


More calculations and further development of a design would certainly be required before definitive statements may be made regarding the ability to mimic the spectrum of ATR in the MATRIX reactors. A main barrier to this is the restrictions that would likely be placed on IPT void reactivity. This places limits on the amount of water that may be present in an IPT since the loop void reactivity is generally positive. Without knowing the maximum amount of water allowable, one may not characterize the flux completely in a test location. However, these preliminary results indicate that the range of fast-to-thermal ratios encountered in ATR is likely to be enveloped by many, if not all, of the MATRIX concepts. The question of whether these can be met while respecting any reactivity insertion limits upon voiding remains for further analysis as work progresses. Thermalization of the spectrum must be performed within the bounds of IPT loop void reactivity requirements. This is addressed for the Cylindrical core in Section 9.6.

\subsection{Power Generated in HEU Test Specimen}

One of the NR requirements listed in Section 1.2 is that the converted ATR achieve greater than $4.8 \times 10^{14}$ fissions per second per gram ${ }^{235} \mathrm{U}$ in a specimen with 1 gram ${ }^{235} \mathrm{U}$ per linear inch in a standard in-pile tube (SE or SW) operating at $60 \mathrm{MW}$. Because there is no clear analog to "lobe power" in the Cylindrical MATRIX concept, this is evaluated by a one-to-one comparison with the ATR model. To accomplish this, a Serpent 2 model was developed where a simple cylinder of HEU fuel was placed at the center of an IPT in the Cylindrical MATRIX. A variation on the ATR model discussed in Section 7.1 was developed that assumed a $0.28615 \mathrm{~cm}$ radius, $60.96 \mathrm{~cm}$ (24")-long cylinder positioned in the center of both SE and SW IPTs. The target mixture was assumed to be the same composition as plate 5 of a fresh ATR HEU (Mark VII) fuel element; Table 6 provides the composition of this cylinder. A total density of 3.94 $\mathrm{g} / \mathrm{cm}^{3}$ of fuel material in the $15.68 \mathrm{~cm}^{3}$ volume of the cylinder gives a total mass of $61.81 \mathrm{~g}$. With the $0.28615 \mathrm{~cm}$ radius, the target contains exactly $1 \mathrm{~g}$ of ${ }^{235} \mathrm{U}$ per inch of height. The HEU plug is surrounded by water.

Calculations were performed to obtain the fission rate averaged over full cylinder volume. In each case, fission rates were calculated in the 24" tall experimental HEU plug in both the SW and SE positions at the point in depletion closest to $k_{\text {eff }}=1$. The HEU plugs were not modeled as depletable, so it was as though a fresh HEU experiment was inserted at each step, affecting flux and reactivity, but not changing in composition with depletion.

The same procedure was followed for the Cylindrical MATRIX model in a peripheral IPT. All dimensions of the HEU plug were the same as in the ATR model. The main difference was that the amount of water surrounding the HEU plug was greater in the Cylindrical model than in the ATR due to the larger flux trap size than the ATR SE and SW flux traps.

It is important to note that the requirement for power in the HEU specimen in ATR is based on a tilted core and a $60 \mathrm{MW}$ lobe. Ability to tilt the power in the MATRIX core will impact the ability to meet this criterion. Therefore, these results should be considered preliminary and the issue should be revisited in future work. 
Table 7-3. Composition of 24" Tall Cylinder of HEU.

\begin{tabular}{|c|c|}
\hline Nuclide & $\begin{array}{c}\text { Atomic Density } \\
\text { (atoms/b-cm) }\end{array}$ \\
\hline${ }^{234} \mathrm{U}$ & $4.2343 \times 10^{-5}$ \\
\hline${ }^{235} \mathrm{U}$ & $3.9211 \times 10^{-3}$ \\
\hline${ }^{236} \mathrm{U}$ & $1.4694 \times 10^{-5}$ \\
\hline${ }^{238} \mathrm{U}$ & $2.3521 \times 10^{-4}$ \\
\hline${ }^{27} \mathrm{Al}$ & $5.1237 \times 10^{-2}$ \\
\hline
\end{tabular}

Table 7-4. Fission rates per gram ${ }^{235} \mathrm{U}$ in prototypic HEU cylinder in ATR and MATRIX.

\begin{tabular}{|c|c|c|}
\hline Reactor & Location & $\begin{array}{c}\text { Fission Rate } \\
\text { (fissions } / \mathbf{s} / \mathbf{g}^{235} \mathbf{U} \text { ) }\end{array}$ \\
\hline \multirow{2}{*}{$\begin{array}{c}\text { ATR } \\
\text { Simplified case } \\
\text { described in Section } 7.1\end{array}$} & SW Flux Trap & $5.22 \times 10^{14}$ \\
\hline & SE Flux Trap & $5.10 \times 10^{14}$ \\
\hline \multirow{2}{*}{$\begin{array}{c}\text { Cylindrical } \\
\text { MATRIX } \\
\text { Baseline Al Rack }\end{array}$} & Peripheral Flux Trap & $5.88 \times 10^{14}$ \\
\hline & Center Flux Trap & $7.05 \times 10^{14}$ \\
\hline
\end{tabular}

\section{Comparisons and Downselection}

At this stage in the analysis, calculations had been performed on the main reactor types and many variations on those baseline cases. From this information, comparisons were and a downselection performed. This narrowing of the field of candidates facilitates the further development and analysis of the selected concept. The criteria used to compare the reactor options were derived essentially from the mission requirements developed in Ref. [5] and summarized in Section 1.2 of this report. The following sections give criteria and comparisons between the MATRIX concepts used in downselection. The numerical scores are tallied in Table $8-5$ on page 80 .

\subsection{Achievable Cycle Length}

The first criterion used was the cycle length requirement. From the comparison to an analogous ATR model described in Section 7, it was determined that the $\mathrm{B}_{1}$ for ATR with no poison, no control material, and a full core of fresh fuel is approximately 56 days. The various concepts 
introduced and analyzed in this report are summarized in Table 8-1. The four baseline concepts (bolded) are given along with the variations on the baseline designs with the varied parameters given in red. It is expected that a replacement reactor should have at least the capability to achieve the same cycle length as the ATR in its current configuration. This characteristic is judged using the $\mathrm{B}_{1}$ value of 56 days on a pass/fail basis in the far-right column of Table 8-1. Burnup in excess of this minimum value of 56 days may be advantageous, but this is not given additional consideration at the present stage of the analysis.

All four of the baseline candidate reactors pass the achievable burnup criterion with their nominal Al-6061 pressure vessels. However, replacement of this pressure vessel material with SS304 causes the Annular and Square concepts to fail the burnup criterion. Only Cylindrical and PBT, as designed, had enough reactivity to allow for the reactivity and burnup penalty of the SS304 vessel and still satisfy the burnup criterion.

The various reflector tank contents studied in Section 4 were only examined for the Cylindrical core. With all other parameters being nominal, all of the reflectors tested with the exception of $\mathrm{H}_{2} \mathrm{O}$ satisfied the burnup criterion. Any of the concepts featuring an aluminum core rack and an Al-6061 pressure vessel would be expected to exhibit a similar reactivity effect for each of the reflector material options. Therefore, other concepts than the Cylindrical were not used to repeat that analysis.

Replacement of the Al-6061 core racks in the Cylindrical and Annular baseline cores with $\mathrm{Al} / \mathrm{Be}$ alloys of increasing beryllium fraction increases the achievable burnup substantially. Because there is no analogous feature in the PBT concept, this analysis is not carried out for that core. Simply replacing the $\mathrm{U}-10 \mathrm{Mo}$ fuel meat with $\mathrm{U}_{3} \mathrm{Si}_{2}$ carries a substantial reactivity and burnup penalty. None of the otherwise-baseline concepts satisfy the burnup criterion with the $\mathrm{U}_{3} \mathrm{Si}_{2}$ alternative fuel except for the PBT core with Al-6061 pressure boundaries. In the Cylindrical and Annular cores, however, addition of beryllium to the core rack facilitates the use of $\mathrm{U}_{3} \mathrm{Si}_{2}$ alternative fuel meat.

Concepts not satisfying the imposed burnup criterion were removed from consideration at this stage. The reduced field of candidate concepts is given in Table 8-2 along with an alphanumeric identifier. This identifier will be used in a later table for convenience as a final comparison table is constructed. In addition to removing the concepts that fail the achievable burnup criterion, the Cylindrical concepts with various reflector contents were removed. This was done to avoid confusion since these reflectors can be judged somewhat independently from the reactors. The exception to this is, of course, the PBT concept, since the shape of the reflector tank is quite different from the other three concepts. 


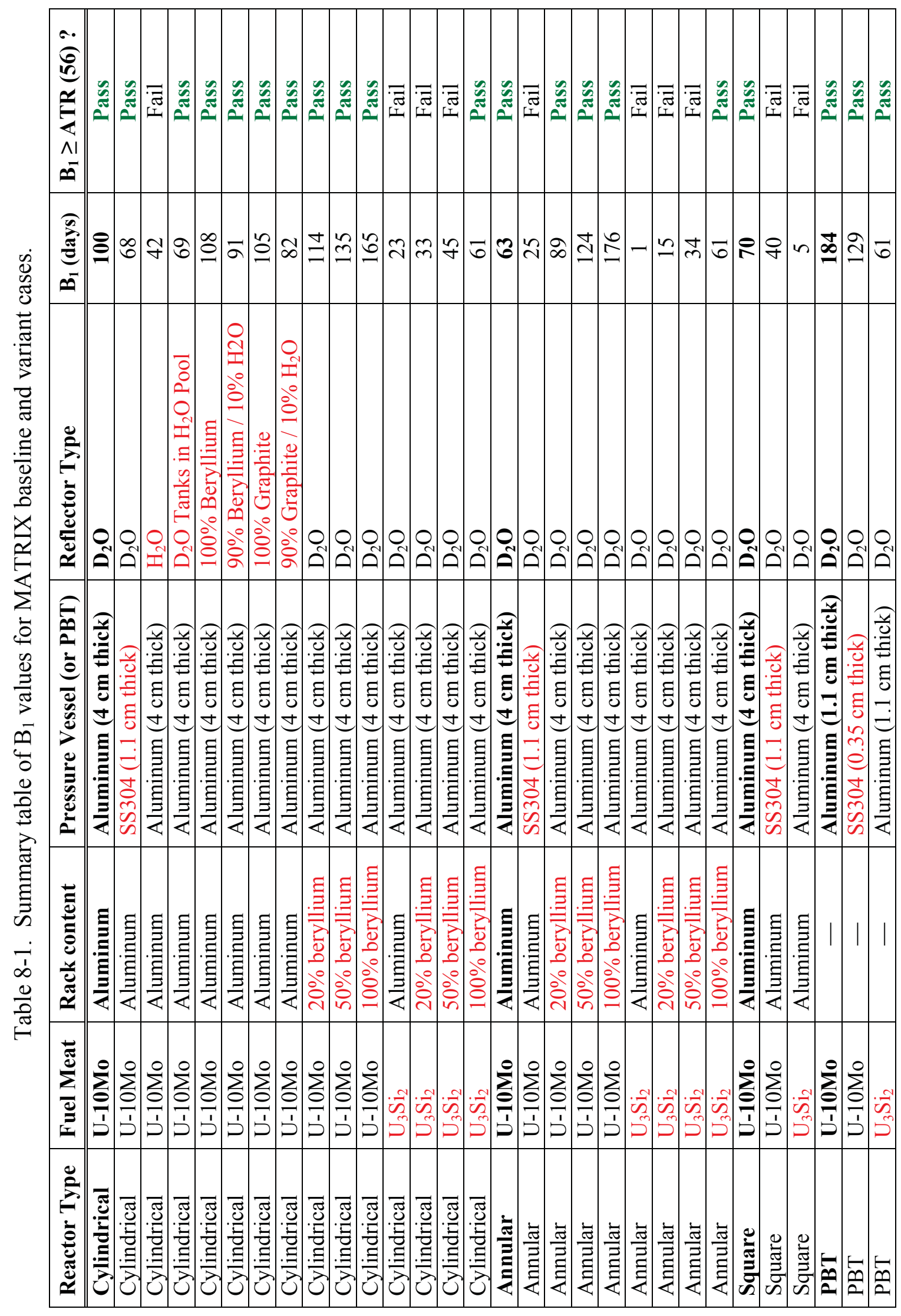


Table 8-2. Reduced field of candidates with alphanumeric identifiers.

\begin{tabular}{|c|c|c|c|c|c|}
\hline Reactor Type & Fuel Meat & Rack content & Pressure Vessel (or PBT) & $\begin{array}{l}\text { Reflector } \\
\text { Type }\end{array}$ & ID \\
\hline Cylindrical & \begin{tabular}{|l} 
U-10Mo \\
\end{tabular} & Aluminum & Aluminum (4 cm thick) & $\overline{\mathbf{D}_{2} \mathrm{O}}$ & $\mathrm{C} 1$ \\
\hline Cylindrical & U-10Mo & Aluminum & SS304 (1.1 cm thick) & $\mathrm{D}_{2} \mathrm{O}$ & $\mathrm{C} 2$ \\
\hline Cylindrical & U-10Mo & $20 \%$ beryllium & Aluminum (4 cm thick) & $\mathrm{D}_{2} \mathrm{O}$ & $\mathrm{C} 3$ \\
\hline Cylindrical & U-10Mo & $50 \%$ beryllium & Aluminum (4 cm thick) & $\mathrm{D}_{2} \mathrm{O}$ & $\mathrm{C} 4$ \\
\hline Cylindrical & U-10Mo & $100 \%$ beryllium & Aluminum (4 cm thick) & $\mathrm{D}_{2} \mathrm{O}$ & $\mathrm{C5}$ \\
\hline Cylindrical & $\mathrm{U}_{3} \mathrm{Si}_{2}$ & $100 \%$ beryllium & Aluminum (4 cm thick) & $\mathrm{D}_{2} \mathrm{O}$ & $\mathrm{C} 6$ \\
\hline Annular & \begin{tabular}{|l|} 
U-10Mo \\
\end{tabular} & Aluminum & Aluminum (4 cm thick) & $\mathrm{D}_{2} \mathrm{O}$ & $\mathrm{A} 1$ \\
\hline Annular & U-10Mo & $20 \%$ beryllium & Aluminum (4 cm thick) & $\mathrm{D}_{2} \mathrm{O}$ & $\mathrm{A} 2$ \\
\hline Annular & \begin{tabular}{|l} 
U-10Mo \\
\end{tabular} & $50 \%$ beryllium & Aluminum (4 cm thick) & $\mathrm{D}_{2} \mathrm{O}$ & $\mathrm{A} 3$ \\
\hline Annular & U-10Mo & $100 \%$ beryllium & Aluminum (4 cm thick) & $\mathrm{D}_{2} \mathrm{O}$ & $\mathrm{A} 4$ \\
\hline Annular & $\mathrm{U}_{3} \mathrm{Si}_{2}$ & $100 \%$ beryllium & Aluminum (4 cm thick) & $\mathrm{D}_{2} \mathrm{O}$ & A5 \\
\hline Square & U-10Mo & Aluminum & Aluminum (4 cm thick) & $\mathbf{D}_{2} \mathbf{O}$ & $\mathrm{S} 1$ \\
\hline PBT & \begin{tabular}{|l|} 
U-10Mo \\
\end{tabular} & NA & Aluminum (1.1 cm thick) & $\mathbf{D}_{2} \mathrm{O}$ & $\mathrm{P} 1$ \\
\hline PBT & U-10Mo & NA & SS304 (0.35 cm thick) & $\mathrm{D}_{2} \mathrm{O}$ & $\mathrm{P} 2$ \\
\hline PBT & $\mathrm{U}_{3} \mathrm{Si}_{2}$ & NA & Aluminum (1.1 cm thick) & $\mathrm{D}_{2} \mathrm{O}$ & $\mathrm{P} 3$ \\
\hline
\end{tabular}

\subsection{High Availability/Capacity Factor}

One of the requirements imposed on the new MATRIX concept is that it has high availability and capacity factor. Though no concrete number is proposed at this point, one may assume that the same or higher availability than the current ATR value would be desirable. Key factors in availability are refueling time and frequency, along with the time and frequency of major maintenance outages. The refueling frequency is dictated by experiment schedules within limits ultimately imposed by the burnup capability of the core. Because the experiment schedules are unknown and the burnup capability was addressed in a separate section, these are not discussed further here.

With regard to refueling time, the MATRIX concepts are at too immature a design stage to make firm assertions as to their relative merits. All of these designs feature a pressure boundary located in close proximity to the fuel. Therefore, replacement of the pressure vessel is likely. No calculations have been made in regard to this. However, the French CEA has stated that for their RJH reactor, a 10-year interval is anticipated for replacement of their aluminum pressure vessel. [13] All but impossible for many existing nuclear reactors, pressure vessel replacement must be planned from the early stages of design for the MATRIX reactor. Lessons learned from the RJH experience should be of value in this process as well. As was shown in Section 5, increasing the moderation from the core rack reduces the fast fluence on the pressure vessel. This may be shown to extend the time between required replacements. However, this has not been quantified at this time.

For routine refueling, speculation on the relative times between the concepts is premature, and so this will be reserved for future work. From both the standpoints of routine refueling and major maintenance outages, no clear advantages could be gleaned from consideration of the 
present state of the MATRIX designs. They are therefore considered equivalent with regard to this criterion.

\subsection{NRC Licensable}

Ability to obtain a construction and operation license from the NRC is an anticipated requirement for MATRIX. This is a significant departure from the DOE-licensed test reactor paradigm of the past. The implications of the various designs on the licensing process have not been analyzed in detail. However, some observations can be made based on available information.

The choice of Al-6061 for the pressure vessel material is arguably the most significant departure from more conventional high-pressure test reactors in the U.S. Currently, ASME pressure vessel codes do not include aluminum alloys at high temperatures normally associated with this type of application. One possible strategy for eliminating this obstacle would be to build a qualification case at higher temperatures. It may be possible to leverage CEA experience in licensing the JHR in this. Another possible strategy would be to provide sufficient cooling such that exceeding the temperatures currently included in the code is precluded.

Because there may be additional cost and possible licensing risk associated with specifying an aluminum pressure vessel, the concepts that include this feature are somewhat disadvantaged in this category. Generally, this makes the Cylindrical and PBT concepts stronger since these are the only two that still meet the burnup criterion with the SS304 pressure vessel in place (albeit with the beryllium core rack option only). Aside from the selection of pressure vessel material, no other discriminating features are known at this time.

\subsection{High Flux in Large Test Volumes}

Perhaps one of the most important performance metrics for a new test reactor would be that it produces high neutron flux in large test volumes. In this section, data generated and reported in

previous sections is condensed and arranged to assess the volume of test locations and the average total flux in those locations. The reduced field of candidates is shown with their usable volumes and average total flux in

Table 8-3. Center and peripheral test locations are handled separately and the usable volume was determined by taking all space within the innermost pipe of the IPTs having the full $120 \mathrm{~cm}$ axial height of active fuel. 
The Cylindrical core and the Square core have the same IPT design with all seven (one central and six peripheral) of them being identical. Each has a usable volume of 8.2 liters, so the peripheral six have a total volume of 48.9 liters. Total flux values averaged over the axial length of the test space are given in

Table 8-3. All of the Cylindrical cases remaining (C1-C6) have comparable flux values in the IPTs. As for the locations inside the fuel assemblies, half of them were assumed to be occupied by shim rods for this calculation. The total remaining volume of these spaces is 17.6 liters. The square core is missing the locations inside assemblies, however other spaces would become available for tests, which are not analyzed in detail here. These concepts are believed therefore to have a strong advantage with regard to this criterion

The Annular and PBT cores share the same IPT design with a larger central location and smaller peripheral locations. The peripheral locations are smaller due to the assumed need for shutdown rods in these locations. The large central location has a volume of 18.2 liters and the sum of the volumes of surrounding peripheral locations is 28.3 liters. Therefore, as currently designed, the Square and Cylindrical designs have much more IPT volume than the Annular and PBT designs. Of course, other irradiation positions are anticipated to be available in all of the concepts.

In the ATR, there were originally nine in-pile tubes in the reactor. In six of these locations, annular safety rods were installed, effectively shrinking the available space for irradiation tests. For the calculation of usable volume in the flux traps in ATR, it was assumed that all of the large flux traps (without shutdown rods) are designed to approximate the NW flux trap and all of the small positions (with shutdown rods) look like the North, West, Southwest, and Southeast flux traps. With this assumption, the usable volume in the small locations totals 11.3 liters and that of the large locations is 29.7 liters. Therefore, all of the designs in 
Table 8-3 have more total usable space in flux traps having IPTs than does the current ATR. From the analysis of the flat ATR core in Section 7, the average flux in the small locations is approximately $1.7 \times 10^{15} \mathrm{n} \cdot \mathrm{cm}^{-2} \cdot \mathrm{s}^{-1}$ while that in the large locations (based on the NW flux trap) is approximately $1.3 \times 10^{15} \mathrm{n} \cdot \mathrm{cm}^{-2} \cdot \mathrm{s}^{-1}$. Note again that this is for a flat core and there would be a great deal of variation based on the loading of fuel and experiments. Therefore, these numbers should be used as a rough estimate. However, from these values, it can be observed that the total flux values in the IPTs of the Cylindrical and Square MATRIX concepts are comparable (if slightly lower in the Annular and PBT designs) to those of ATR. In practice, higher fluxes are routinely achieved in ATR by tilting the power. Capability for power tilts is anticipated, but not yet analyzed thoroughly, in the MATRIX designs as well. Therefore this simplified comparison approach is used.

As is anticipated with the MATRIX designs, there are many more usable locations in the ATR for irradiation experiments. Because the number and size of these locations in MATRIX concepts have not been determined yet, they are omitted from this simplified comparison. Note that this is an important figure of merit to continually revisit as designs develop. From this comparison, the conclusion is drawn that the MATRIX designs do not have a distinct advantage in terms of magnitude of total neutron flux in test locations. However, their fluxes are comparable (particularly in the Cylindrical and Square concepts) and they all appear to have a significant volume advantage over ATR. Among the MATRIX concepts, the Cylindrical and Square concepts are thus considered to be at a strong advantage with the Annular core being neutral and the PBT core having a slight disadvantage.

Table 8-3. Summary table of average total neutron fluxes and volumes of test spaces.

\begin{tabular}{|c|c|c|c|c|c|c|}
\hline \multirow{2}{*}{ ID } & \multicolumn{2}{|c|}{ Center Test Location } & \multicolumn{2}{|c|}{ Peripheral Test Location } & \multicolumn{2}{|c|}{ Inside Fuel Assembly } \\
\hline & $\begin{array}{l}\text { Volume } \\
\text { (liters) }\end{array}$ & $\begin{array}{c}\text { Ave. Flux } \\
\left(\mathrm{n} \cdot \mathrm{cm}^{-2} \cdot \mathbf{s}^{-1}\right)\end{array}$ & $\begin{array}{l}\text { Volume } \\
\text { (liters) }\end{array}$ & $\begin{array}{c}\text { Ave. Flux } \\
\left(n \cdot \mathbf{c m}^{-2} \cdot \mathbf{s}^{-1}\right)\end{array}$ & $\begin{array}{c}\text { Volume } \\
\text { (liters) }\end{array}$ & $\begin{array}{c}\text { Ave. Flux } \\
\left(\mathrm{n} \cdot \mathrm{cm}^{-2} \cdot \mathrm{s}^{-1}\right)\end{array}$ \\
\hline$\overline{\mathrm{C} 1}$ & 8.2 & $1.55 \mathrm{E}+15$ & 48.9 & $1.36 \mathrm{E}+15$ & $17.6^{\mathrm{a}}$ & $1.62 \mathrm{E}+15$ \\
\hline $\mathrm{C} 2$ & 8.2 & $1.73 \mathrm{E}+15$ & 48.9 & $1.40 \mathrm{E}+15$ & 17.6 & $1.78 \mathrm{E}+15$ \\
\hline $\mathrm{C} 3$ & 8.2 & $1.53 \mathrm{E}+15$ & 48.9 & $1.32 \mathrm{E}+15$ & 17.6 & $1.58 \mathrm{E}+15$ \\
\hline $\mathrm{C} 4$ & 8.2 & $1.51 \mathrm{E}+15$ & 48.9 & $1.31 \mathrm{E}+15$ & 17.6 & $1.55 \mathrm{E}+15$ \\
\hline
\end{tabular}

\footnotetext{
${ }^{a}$ Assumes half of the fuel assemblies are unavailable for experiment volume.
} 


\begin{tabular}{|c|c|c|c|c|c|c|}
\hline C5 & 8.2 & $1.44 \mathrm{E}+15$ & 48.9 & $1.29 \mathrm{E}+15$ & 17.6 & $1.51 \mathrm{E}+15$ \\
\hline C6 & 8.2 & $1.54 \mathrm{E}+15$ & 48.9 & $1.38 \mathrm{E}+15$ & 17.6 & $1.78 \mathrm{E}+15$ \\
\hline A1 & 18.2 & $1.56 \mathrm{E}+15$ & 28.3 & $9.57 \mathrm{E}+14$ & NA & NA \\
\hline A2 & 18.2 & $1.56 \mathrm{E}+15$ & 28.3 & $9.32 \mathrm{E}+14$ & NA & NA \\
\hline A3 & 18.2 & $1.53 \mathrm{E}+15$ & 28.3 & $9.11 \mathrm{E}+14$ & NA & NA \\
\hline A4 & 18.2 & $1.40 \mathrm{E}+15$ & 28.3 & $8.86 \mathrm{E}+14$ & NA & NA \\
\hline A5 & 18.2 & $1.54 \mathrm{E}+15$ & 28.3 & $9.49 \mathrm{E}+14$ & NA & NA \\
\hline S1 & 8.2 & $1.52 \mathrm{E}+15$ & 48.9 & $1.36 \mathrm{E}+15^{\text {a }}$ & NA & NA \\
\hline P1 & 18.2 & $1.17 \mathrm{E}+15$ & 28.3 & $8.44 \mathrm{E}+14$ & NA & NA \\
\hline P2 & 18.2 & $1.23 \mathrm{E}+15$ & 28.3 & $8.30 \mathrm{E}+14$ & NA & NA \\
\hline P3 & 18.2 & $1.31 \mathrm{E}+15$ & 28.3 & $9.09 \mathrm{E}+14$ & NA & NA \\
\hline
\end{tabular}

\subsection{Fast Spectrum Capabilities}

Table 8-4 summarizes the peak fast flux values in IPT locations of the MATRIX concepts (and inner-assembly locations of the Cylindrical design). The center IPT locations in the matrix designs have peak fast flux values ranging from $2.2 \times 10^{14}$ to $3.7 \times 10^{14} \mathrm{n} \cdot \mathrm{cm}^{-2} \cdot \mathrm{s}^{-1}$ with the higher values occurring in some of the Cylindrical concepts. The same relative trend between concepts can be observed in the peripheral IPTs, albeit at reduced magnitudes. In the calculations performed on the simplified ATR, the fast flux values in the small and large IPTs yielded fast flux values of $3.5 \times 10^{14}$ and $2.9 \times 10^{14} \mathrm{n} \cdot \mathrm{cm}^{-2} \cdot \mathrm{s}^{-1}$, respectively. These were taken in the SW and NW flux traps, respectively and both were assumed to be aluminum filled like in the MATRIX concepts. As with the average total flux values the IPTs of the MATRIX concept show no distinct advantage over ATR with regard to peak fast flux. This is because fast flux in a test location is largely determined by power density in fuel and proximity to said fuel. These features are essentially common among the MATRIX and ATR concepts.

As mentioned before, the ATR does have a number of other irradiation locations, some of which have higher fast flux than in the IPTs. The A-holes of ATR, for example, are drilled out of the inner beryllium reflector and are quite close in proximity to fuel elements. From Table 7-2, one sees that the peak fast flux in the A-1 hole for the simplified flat core modeled was found to be approximately $4.4 \times 10^{14} \mathrm{n} \cdot \mathrm{cm}^{-2} \cdot \mathrm{s}^{-1}$. None of the IPTs of MATRIX designs show a value this high. However, the locations inside the fuel elements of the Cylindrical design have fast flux somewhat higher than this. The total volume of these locations (again assuming half are unavailable) is 17.6 liters. There are 16 A-holes in ATR. Four of them are larger having a diameter of $1.95 \mathrm{~cm}$, and 12 of them smaller having a diameter of $1.59 \mathrm{~cm}$. The total volume of the A-hole space combined is therefore 4.4 liters. If we assume that the eight small B-holes (diameter $=2.22 \mathrm{~cm}$ ) can achieve a similar fast flux due to their close proximity to fuel, and we add their volume to that of the A-holes, one arrives at a volume of 8.2 liters of space having the peak fast flux of between $4 \times 10^{14}$ and $5 \times 10^{14} \mathrm{n} \cdot \mathrm{cm}^{-2} \cdot \mathrm{s}^{-1}$. This is still substantially less than that of the inter-assembly space in the Cylindrical design, even if fewer than half of these spaces are available for experiments.

Thus it is concluded that in the comparison of available fast flux, the Cylindrical core holds an advantage over ATR and the other MATRIX concepts. The Cylindrical design has

\footnotetext{
${ }^{a} \mathrm{NE}$ flux trap
} 
comparable fast flux in IPTs to those of ATR, but with a larger volume of usable space. Furthermore, the test space inside fuel assemblies provides a larger volume of very-high fast flux irradiation space than those of ATR. The Inter-assembly test spaces are absent in the other MATRIX concepts, which lack a dedicated space with higher fast flux than their IPTs.

Table 8-4. Summary table of peak fast fluxes and volumes of test spaces.

\begin{tabular}{|c|c|c|c|c|c|c|}
\hline & \multicolumn{2}{|c|}{ Center Test Location } & \multicolumn{2}{l|}{ Peripheral Test Location } & \multicolumn{2}{|c|}{ Inside Fuel Assembly } \\
\cline { 2 - 7 } ID & $\begin{array}{c}\text { Volume } \\
\text { (liters) }\end{array}$ & $\begin{array}{c}\text { Peak Fast } \\
\text { Flux } \\
\left(\mathbf{n} \cdot \mathbf{c m}^{-2} \cdot \mathbf{s}^{-1}\right)\end{array}$ & $\begin{array}{c}\text { Volume } \\
(\text { liters })\end{array}$ & $\begin{array}{c}\text { Peak Fast } \\
\text { Flux } \\
\left(\mathbf{n} \cdot \mathbf{c m}^{-2} \cdot \mathbf{s}^{-1}\right)\end{array}$ & $\begin{array}{c}\text { Volume } \\
(\text { liters })\end{array}$ & $\begin{array}{c}\text { Peak Fast } \\
\text { Flux } \\
\left(\mathbf{n} \cdot \mathbf{c m}^{-2} \cdot \mathbf{s}^{-1}\right)\end{array}$ \\
\hline C1 & 8.2 & $3.22 \mathrm{E}+14$ & 48.9 & $2.79 \mathrm{E}+14$ & $17.6^{\text {a }}$ & $5.07 \mathrm{E}+14$ \\
\hline C2 & 8.2 & $3.67 \mathrm{E}+14$ & 48.9 & $2.94 \mathrm{E}+14$ & 17.6 & $5.73 \mathrm{E}+14$ \\
\hline C3 & 8.2 & $3.12 \mathrm{E}+14$ & 48.9 & $2.69 \mathrm{E}+14$ & 17.6 & $4.91 \mathrm{E}+14$ \\
\hline C4 & 8.2 & $2.92 \mathrm{E}+14$ & 48.9 & $2.59 \mathrm{E}+14$ & 17.6 & $4.86 \mathrm{E}+14$ \\
\hline C5 & 8.2 & $2.68 \mathrm{E}+14$ & 48.9 & $2.41 \mathrm{E}+14$ & 17.6 & $4.74 \mathrm{E}+14$ \\
\hline C6 & 8.2 & $2.78 \mathrm{E}+14$ & 48.9 & $2.52 \mathrm{E}+14$ & 17.6 & $4.91 \mathrm{E}+14$ \\
\hline A1 & 18.2 & $2.99 \mathrm{E}+14$ & 28.3 & $1.87 \mathrm{E}+14$ & NA & NA \\
\hline A2 & 18.2 & $3.10 \mathrm{E}+14$ & 28.3 & $1.83 \mathrm{E}+14$ & NA & NA \\
\hline A3 & 18.2 & $2.98 \mathrm{E}+14$ & 28.3 & $2.20 \mathrm{E}+14$ & NA & NA \\
\hline A4 & 18.2 & $2.70 \mathrm{E}+14$ & 28.3 & $1.99 \mathrm{E}+14$ & NA & NA \\
\hline A5 & 18.2 & $2.86 \mathrm{E}+14$ & 28.3 & $1.89 \mathrm{E}+14$ & NA & NA \\
\hline S1 & 8.2 & $2.71 \mathrm{E}+14$ & 48.9 & $2.68 \mathrm{E}+14$ & NA & NA \\
\hline P1 & 18.2 & $2.23 \mathrm{E}+14$ & 28.3 & $1.68 \mathrm{E}+14$ & NA & NA \\
\hline P2 & 18.2 & $2.99 \mathrm{E}+14$ & 28.3 & $1.68 \mathrm{E}+14$ & NA & NA \\
\hline P3 & 18.2 & $2.41 \mathrm{E}+14$ & 28.3 & $1.68 \mathrm{E}+14$ & NA & NA \\
\hline
\end{tabular}

\subsection{Modularity of Core}

Another figure of merit in judging a replacement test reactor is the modularity and flexibility of the core. As has been the case during the 50+ year lifetime of ATR, the missions of a reactor can change substantially. Through the creativity of its reactor engineers and physicists, ATR was adapted to its changing mission with a high degree of success. Nevertheless, any new reactor should be designed with adaptability in mind.

The $\mathrm{D}_{2} \mathrm{O}$ tank nominally designed in all of the concepts is anticipated to provide a very high degree of flexibility to both the initial designers and to the custodians of the machine for its lifetime. Being at or near atmospheric pressure should allow for rather straightforward modifications of the contents of the tank, although the presence of $\mathrm{D}_{2} \mathrm{O}$ presents some operational complications in its need to be sealed from the surrounding environment. The alternative reflector tank concepts discussed in Section 5 could relieve some of this, however these alternatives are rather concept-neutral with the exception of the PBT design.

\footnotetext{
${ }^{a}$ Assumes half of the fuel assemblies are unavailable for experiment volume.
} 
The main differences in flexibility would likely arise from the reactor components inside the pressure boundary. Among the MATRIX candidate designs, the Square and Cylindrical possess the greatest capability for adaptation to changing missions inside the pressure vessel. The core rack should be designed such that a fuel location can accept either a fuel assembly or a test train of the same size. Reactor control hardware, such as control or shim rods, should be designed to be movable such that they can be placed in locations of appropriate worth for various configurations.

On the other hand, it cannot be ruled out that a fuel assembly could be removed and replaced with a test train in the Annular and PBT cores. These concepts have arcuate assemblies similar to those of ATR, which may be more difficult to replace with non-fuel, though this should not be considered impossible at this point. Because of this uncertainty, only a slight advantage is assigned to the Square and Cylindrical concepts based on this criterion.

\subsection{Online Experiment Access and Rabbits}

The capability to insert and remove experiments during operation can provide substantial advantages and expansion of the option space for simultaneous reactor missions. Pneumatically driven "rabbits" have been used for decades to provide access to test locations in reactors, including ATR, during operation. These can be designed to cross high-pressure boundaries, although it increases cost and complexity somewhat. At the current stage of design of the MATRIX concepts, no significant differences are known that would render one design or another more advantageous over others with regard to access to experiments within the pressure boundary.

The reflector tanks of all of the MATRIX designs are to be constructed such that they provide a large low-pressure environment with online access to irradiation spaces. Because all of the concepts call for this, no differences are known between the MATRIX concepts with regard to this criterion. The primary differences in online experiment access between the ideas presented in this report likely arise from the selection of the reflector tank content; the reference $\mathrm{D}_{2} \mathrm{O}$ or one of the alternatives presented in Section 4. For example, the option of an open-top water tank with $\mathrm{D}_{2} \mathrm{O}$ cans inside may offer an attractive combination of high flux and easy access. However, because this option is concept neutral (with the exception of the PBT core), it is not considered a determining factor here.

\subsection{Minimum waste stream effluents/environmental impact}

Minimization of waste streams and reducing environmental impact are important objectives of a new reactor concept. With regard to fuel, all of the MATRIX options have been designed to be very similar, differing primarily in the geometry of elements. Thus no significant differences in waste stream are anticipated in relation to fuel.

The $\mathrm{D}_{2} \mathrm{O}$ reflector tank specified for all of the baseline reactor concepts will share the environmental issue of tritium production. The production rate has yet to be quantified, but must be accounted for since this is a high flux environment and many neutron captures are anticipated. The PBT concepts contain more $\mathrm{D}_{2} \mathrm{O}$ in a higher-flux environment than the other designs. Therefore, these are likely to be the most prolific producers of tritium. For this reason, they are considered to be at a slight disadvantage compared to the other three concepts. Among the other 
candidates, the $\mathrm{D}_{2} \mathrm{O}$ tank is roughly the same size and positioned the same with respect to the core. Therefore, no substantial difference between these is known at this time.

The baseline cases of each reactor have omitted beryllium as a major material in the core. This is considered favorable due to the beryllium disposal difficulties that have been encountered in ATR operation over the years. Variations on the Cylindrical and Annular designs that contain aluminum/beryllium alloy core racks introduce beryllium into the waste stream. Therefore, these are considered to be at a slight disadvantage compared to the concepts that do not contain beryllium.

\subsection{Imaging, Neutron Scattering}

The secondary mission of imaging and neutron scattering experimentation is facilitated by high thermal neutron flux outside the core. In all of the MATRIX concepts, the idea behind the large $\mathrm{D}_{2} \mathrm{O}$ tank is to facilitate high thermal flux outside the main pressure boundary. This provides a reconfigurable space with relatively straightforward access for these types of secondary missions. Because the PBT concepts have larger $\mathrm{D}_{2} \mathrm{O}$ tanks than the other three main types, these are considered to be at a slight advantage with regard to this criterion.

\subsection{Radioisotope Production}

With regard to radioisotope production, high flux and ease of access are two of the most important features one could desire in a reactor design. For this reason, a similar comparison exists to that related to imaging and neutron scattering. All of the MATRIX concepts feature the large $\mathrm{D}_{2} \mathrm{O}$ tank outside the pressure boundary. Therefore, the Square, Cylindrical, and Annular concepts are considered equivalent in this regard. Again, because the PBT concepts have larger $\mathrm{D}_{2} \mathrm{O}$ tanks than the other three main types, these are considered to be at a slight advantage with regard to this criterion.

\subsection{Comparison Summary}

Table 8-5 shows a summary of downselection criteria and the scores given to the remaining field of candidates. The Cylindrical concepts receive the highest total scores among the MATRIX concepts. This is the baseline design, therefore, that is carried forward for further design and analysis.

Table 8-5. Summary table of downselection criteria and scores.

\begin{tabular}{|l||c|c|c|c|c|c|c|c|c|c|c|c|c|c|c|}
\hline Criterion & C1 & C2 & C3 & C4 & C5 & C6 & A1 & A2 & A3 & A4 & A5 & S1 & P1 & P2 & P3 \\
\hline $\begin{array}{l}\text { Availability / } \\
\text { Capacity Factor }\end{array}$ & 3 & 3 & 3 & 3 & 3 & 3 & 3 & 3 & 3 & 3 & 3 & 3 & 3 & 3 & 3 \\
\hline NRC Licensable & 2 & 3 & 2 & 2 & 2 & 2 & 2 & 2 & 2 & 2 & 2 & 2 & 2 & 3 & 2 \\
\hline
\end{tabular}




\begin{tabular}{|l||c|c|c|c|c|c|c|c|c|c|c|c|c|c|c|}
\hline $\begin{array}{l}\text { High Flux in Large } \\
\text { Volume }\end{array}$ & 4 & 4 & 4 & 4 & 4 & 4 & 3 & 3 & 3 & 3 & 3 & 4 & 2 & 2 & 2 \\
\hline $\begin{array}{l}\text { Fast Spectrum } \\
\text { Capability }\end{array}$ & 5 & 5 & 5 & 5 & 5 & 5 & 3 & 3 & 3 & 3 & 3 & 3 & 3 & 3 & 3 \\
\hline $\begin{array}{l}\text { Modularity of } \\
\text { Core }\end{array}$ & 4 & 4 & 4 & 4 & 4 & 4 & 3 & 3 & 3 & 3 & 3 & 4 & 3 & 3 & 3 \\
\hline $\begin{array}{l}\text { Online Experiment } \\
\text { Access }\end{array}$ & 3 & 3 & 3 & 3 & 3 & 3 & 3 & 3 & 3 & 3 & 3 & 3 & 3 & 3 & 3 \\
\hline $\begin{array}{l}\text { Minimum Waste / } \\
\text { Environ. Impact }\end{array}$ & 3 & 3 & 2 & 2 & 2 & 2 & 3 & 2 & 2 & 2 & 2 & 3 & 2 & 2 & 2 \\
\hline $\begin{array}{l}\text { Imaging / Neutron } \\
\text { Scattering }\end{array}$ & 3 & 3 & 3 & 3 & 3 & 3 & 3 & 3 & 3 & 3 & 3 & 3 & 4 & 4 & 4 \\
\hline $\begin{array}{l}\text { Radioisotope } \\
\text { Production }\end{array}$ & 3 & 3 & 3 & 3 & 3 & 3 & 3 & 3 & 3 & 3 & 3 & 3 & 4 & 4 & 4 \\
\hline $\begin{array}{l}\text { TOTAL } \\
\text { TOTAL }\end{array}$ & 31 & 29 & 29 & 29 & 29 & 26 & 25 & 25 & 25 & 25 & 28 & 26 & 27 & 26 \\
\hline
\end{tabular}

$1=$ Significant Disadvantage

$2=$ Slight Disadvantage

$3=$ Neutral

$4=$ Slight Advantage

$5=$ Significant Advantage

\section{Further Design Considerations for Cylindrical Concept}

\subsection{Potential for PWR Fuel Pin Irradiations in Reflector Tank}

The potential for various types of irradiation experiments in the reflector tank is briefly addressed in this section. The design of the JHR specifies PWR-type fuel irradiation experiments in pressurized tubes in the reflector tank. This arrangement could provide flexibility as well as offer a large amount of space for multiple irradiations. The JHR design also calls for tests that can be moved radially to simulate transients. [13] In this section, calculations are described that give a preliminary evaluation of what linear power can be achieved in a test such as this in the MATRIX reactors.

The baseline Cylindrical core was used for this analysis. Conceptual test hardware was devised by changing the radii of the IPTs such that a PWR pin having radius $0.41 \mathrm{~cm}$ can fit inside without a great deal of additional space. The resulting dimensions are shown in Figure 9-1. Note that this is a concept borrowing from approximate dimensions from ATR and JHR and has not been analyzed for structural integrity, etc. It is only meant for preliminary reactor physics scoping calculations. The fuel pin was assumed to be composed of $4.5 \mathrm{w} / \mathrm{o}$ enriched $\mathrm{UO}_{2}$ fuel having the nuclide densities shown in Table 9-1. The height of the pin was assumed to be $60.96 \mathrm{~cm}(24$ ") centered at the axial center of the axial core. This amounts to approximately half of the core height. Cladding was ignored for this calculation, a simplification that would not be expected to change the results of this calculation drastically. The cooling water surrounding the pin and just outside the flow tube is assumed to be at typical PWR conditions $\left(\rho=741 \mathrm{~g} / \mathrm{cm}^{3}\right.$, $\mathrm{T}=580 \mathrm{~K}$ ). The pin temperature is assumed to be $1500 \mathrm{~K}$ with a flat temperature profile. The 
calculation was run for distances between the outer wall of the vessel and the center of the PWR fuel test ranging from 6 to $36 \mathrm{~cm}$. Figure 9-2 shows a screen capture of the Serpent model containing the PWR pin test in the reflector at $6 \mathrm{~cm}$ from the vessel wall, the closest position evaluated here.

The resulting linear power values for the PWR pin test are given in Table 9-2 along with the core reactivity for each case. A case with no test hardware in the reflector at all is given at the bottom of the table. Linear powers for the PWR pin test range from $104-210 \mathrm{~kW} / \mathrm{m}$ for the configurations tested here. Typical average linear power in a PWR is $17.8 \mathrm{~kW} / \mathrm{m}$ with a typical peak value of $42.7 \mathrm{~kW} / \mathrm{m}$. [18] Therefore, it appears that typical PWR linear power values can be achieved in this type of arrangement. Some of the alternative reflectors evaluated in Section 4 have lower flux values than the $\mathrm{D}_{2} \mathrm{O}$ reflector used in this analysis. However, even a reduction by a factor of two in flux should still facilitate prototypic PWR power densities in this test arrangement. Other measures may be possible to increase the potential power density of a reflector PWR test, such as tilting the core power, etc.

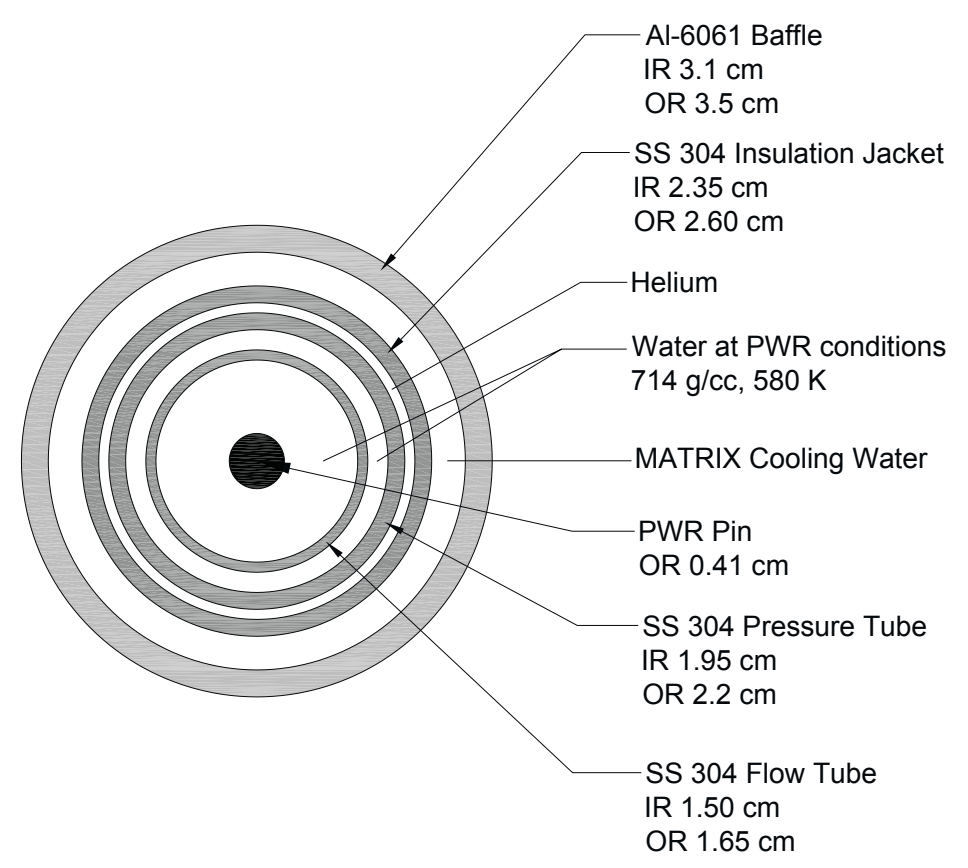

Figure 9-1. Reflector PWR pin irradiation hardware dimensions and materials.

Table 9-1. Composition of 24" Tall Cylinder of PWR fuel. 


\begin{tabular}{|c|c|}
\hline Nuclide & $\begin{array}{c}\text { Atomic Density } \\
\text { (atoms/b-cm) }\end{array}$ \\
\hline${ }^{235} \mathrm{U}$ & $1.056 \times 10^{-3}$ \\
\hline${ }^{238} \mathrm{U}$ & $2.215 \times 10^{-2}$ \\
\hline${ }^{16} \mathrm{O}$ & $4.461 \times 10^{-2}$ \\
\hline
\end{tabular}

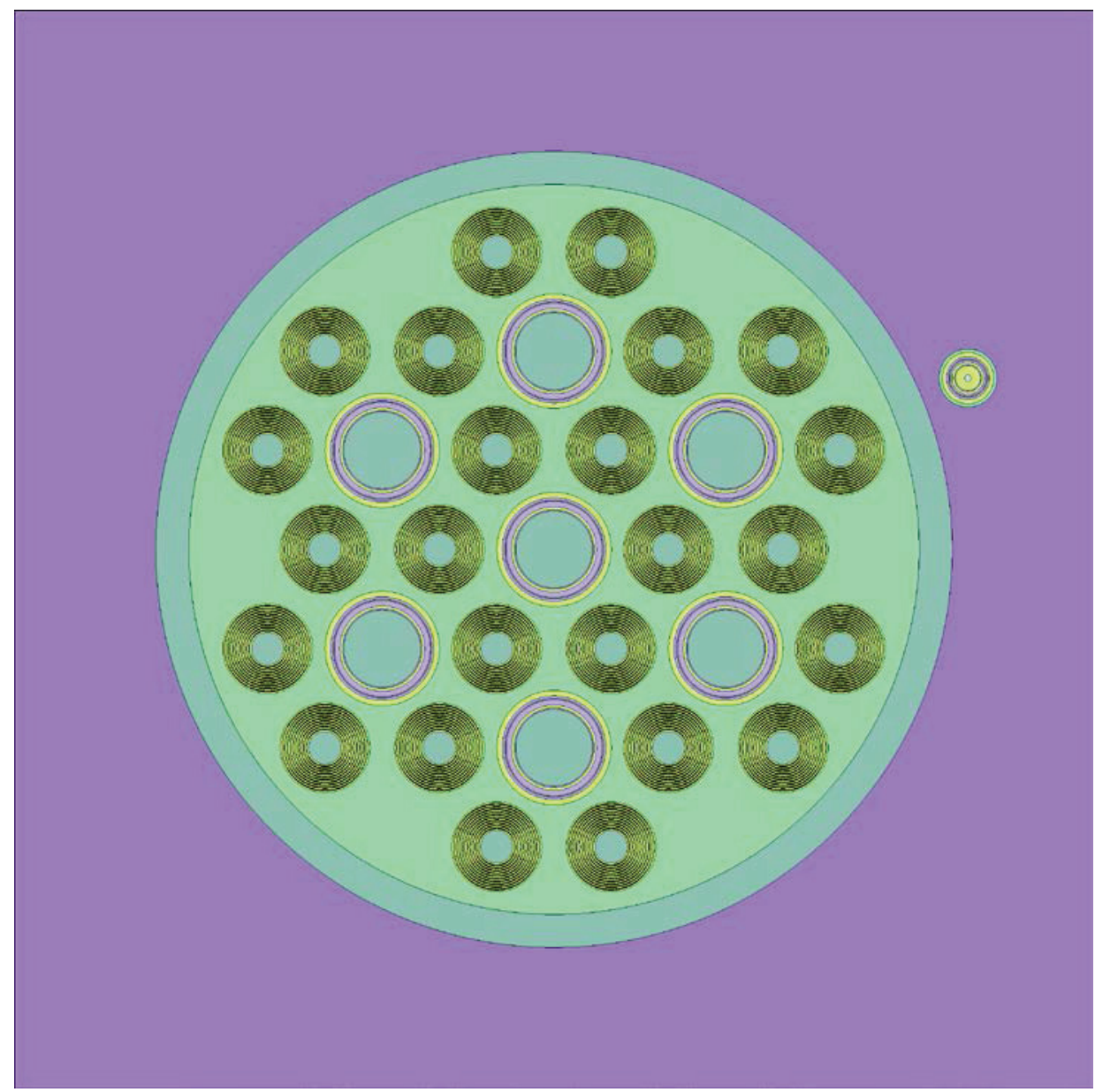

Figure 9-2. Baseline Cylindrical core model with PWR irradiation apparatus in reflector.

Table 9-2. Reactivity and linear power of PWR fuel pin test in reflector of baseline Cylindrical in core.

\begin{tabular}{|c|c|c|c|c|}
\hline \multirow{2}{*}{$\begin{array}{c}\text { D1 } \\
(\mathrm{cm})\end{array}$} & $\begin{array}{c}\mathrm{D2}^{\mathrm{b}} \\
(\mathrm{cm})\end{array}$ & $\begin{array}{c}\text { Initial } \\
k_{\text {eff }}\end{array}$ & \multicolumn{2}{|c|}{ Linear Power in PWR Fuel Pin } \\
\cline { 3 - 5 } & & $\mathrm{kW} / \mathrm{ft}$ & $\mathrm{kW} / \mathrm{m}$ \\
\hline
\end{tabular}

${ }^{\mathrm{a}} \mathrm{D} 1=$ Distance from core center to PWR pin sample center.

${ }^{\mathrm{b}} \mathrm{D} 2$ = Distance from outer pressure vessel surface to PWR pin sample center. 


\begin{tabular}{|c|c|c|c|c|}
\hline 54 & 6 & $1.18040 \pm 0.00015$ & 64.1 & 210 \\
\hline 64 & 16 & $1.18179 \pm 0.00015$ & 54.8 & 180 \\
\hline 74 & 26 & $1.18239 \pm 0.00015$ & 42.6 & 140 \\
\hline 84 & 36 & $1.18294 \pm 0.00015$ & 31.7 & 104 \\
\hline \multicolumn{2}{|l|}{ No PWR Pin Test } & $1.18448 \pm 0.00015$ & & \\
\hline
\end{tabular}

Table 9-3 shows analogous results, except with the SS304 pressure vessel described in Section 3. From these results, one sees that with the power in the PWR fuel pin in the reflector tank is reduced, although not to an extreme degree. This is expected since the results of tallies reported previously show that the flux values are not diminished greatly by using the thinner SS304 vessel.

Table 9-3. Reactivity and linear power of PWR fuel pin test in reflector of Cylindrical core with SS304 pressure vessel.

\begin{tabular}{|c|c|c|c|c|}
\hline \multirow{2}{*}{$\begin{array}{l}\mathrm{D1}^{\mathrm{a}} \\
(\mathrm{cm})\end{array}$} & \multirow{2}{*}{$\begin{array}{l}\mathrm{D}^{\mathrm{b}} \\
(\mathrm{cm})\end{array}$} & \multirow{2}{*}{$\begin{array}{c}\text { Initial } \\
\boldsymbol{k}_{\text {eff }}\end{array}$} & \multicolumn{2}{|c|}{ Linear Power in PWR Fuel Pin } \\
\hline & & & $\mathbf{k W} / \mathbf{f t}$ & $\mathbf{k W} / \mathbf{m}$ \\
\hline 54 & 9 & $1.13678 \pm 0.00015$ & 56.0 & 184 \\
\hline 64 & 19 & $1.13669 \pm 0.00015$ & 45.3 & 150 \\
\hline 74 & 29 & $1.13669 \pm 0.00015$ & 34.6 & 114 \\
\hline 84 & 39 & $1.13730 \pm 0.00015$ & 25.7 & 85 \\
\hline \multicolumn{2}{|c|}{ No PWR Pin Test } & $1.13797 \pm 0.00015$ & & \\
\hline
\end{tabular}

\subsection{Representative Loadings and Power Tilts}

In this section, preliminary consideration is given to prototypic core loadings in the Cylindrical core. First, an initial 3-batch loading was devised giving an approximately even distribution of power across the core. Note that this pattern was not optimized, but merely represents a starting point toward a flat core loading. The loading pattern is shown in Figure 9-3 with fresh, once-burned, and twice burned fuel represented by $F, 1 B$, and 2B, respectively. Once and twice-burned fuel compositions were determined using a Serpent depletion calculation where an entire core of fresh fuel was loaded. This was depleted for 50 days at $250 \mathrm{MW}$, followed by 46 days zero-power decay, then burned for another 50 days at $250 \mathrm{MW}$, then shut down again and decayed 46 days more. Once-burned fuel compositions were taken from the end of the first 46-day decay and Twice-burned compositions taken from the end of the second 46-

${ }^{\mathrm{a}} \mathrm{D} 1=$ Distance from core center to PWR pin sample center.

${ }^{\mathrm{b}} \mathrm{D} 2$ = Distance from outer pressure vessel surface to PWR pin sample center. 
day decay. These shutdown times are not based on exact expectations of operational needs, but assumed based on the need for some decay between cycles. The initial $k_{\text {eff }}$ of this core is 1.07689 and the cycle length was determined to be 50 days. This is expected from a 3-batch core with $\mathrm{B}_{1} \sim 100$ days if linear reactivity assumptions are made.

The ATR is routinely expected to achieve significant power tilting across its lobes in order to support irradiations of different intensities at the same time. ATR is capable of achieving a power tilt of greater than a 3:1 ratio from its SE to NW lobes, albeit at lower than its rated thermal power. [19] It is anticipated that in a MATRIX design, similar power-tilt capabilities will be required, though the "lobe" concept may not apply. Therefore, some simple studies of achieving power tilt through 3-batch core loading were performed. In addition to the prototypic 3-batch loading discussed previously and shown in Figure 9-3, four other loadings were modeled. These are shown in Figure 9-4. Starting from the prototypic flat core, the core labeled "Tilt 1" swaps two assemblies in such a way that concentrates power near the NW flux trap at the expense of power near the SE flux trap. Each subsequent tilted core thereafter results from swapping two more assemblies.

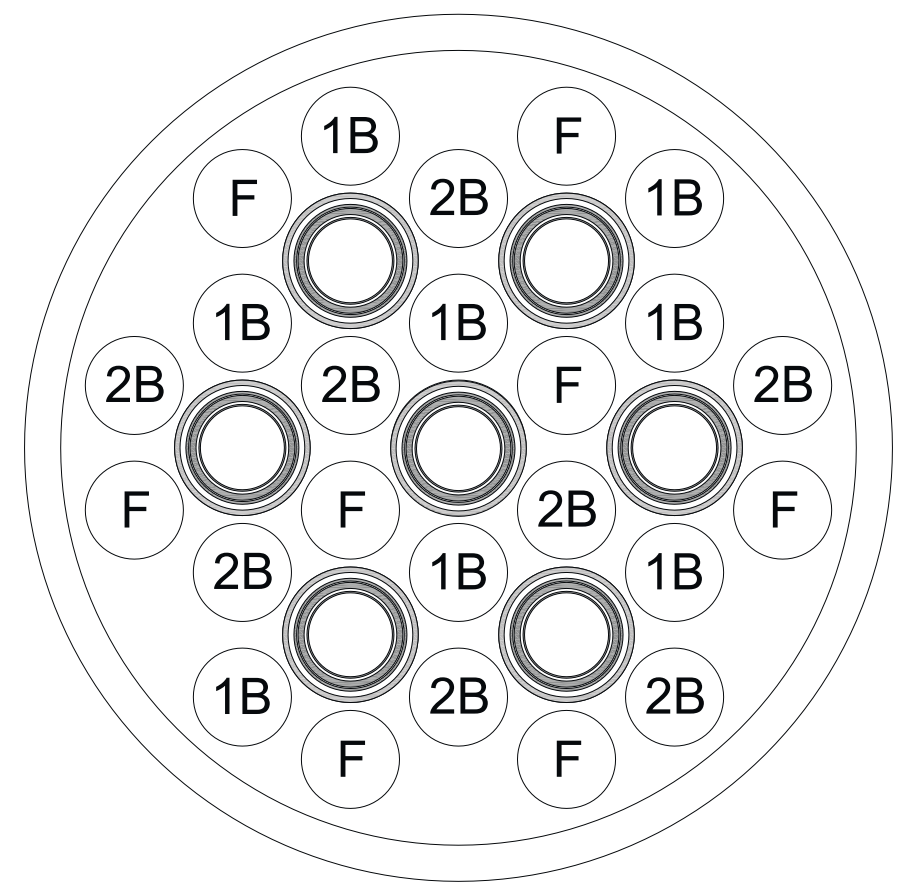

Figure 9-3. Assembly layout of prototypic flat 3-batch Cylindrical core.

Table 9-4 gives the $k_{\text {eff }}$, total neutron flux in the NW and SE flux traps, and the ratio of these fluxes for the prototypic flat case along with the four tilted core loadings. This shows that concentrating fresh assemblies together in the way shown has increased reactivity somewhat. This is, in part, due to the original location of fresh assemblies being more on the periphery of the reactor. Because the reactor is not kept critical for this analysis, the magnitude of the flux is not to be considered exact, however this gives the designer information as to how much tilt can be achieved with core loading alone. With each successive swap of assemblies, the ratio of total neutron flux from SE to NW increases less, until in the case labeled Tilt 4 it has reached a ratio 
of 2.0. Although it is not presently known how much tilt can be achieved in ATR with fuel assembly loading alone, control drums are also used in order to achieve this tilt. For this particular MATRIX concept, it appears that some type of shim would need to be used in order to augment the loading-driven tilt in order to achieve the 3:1 ratio that would be anticipated as a requirement.

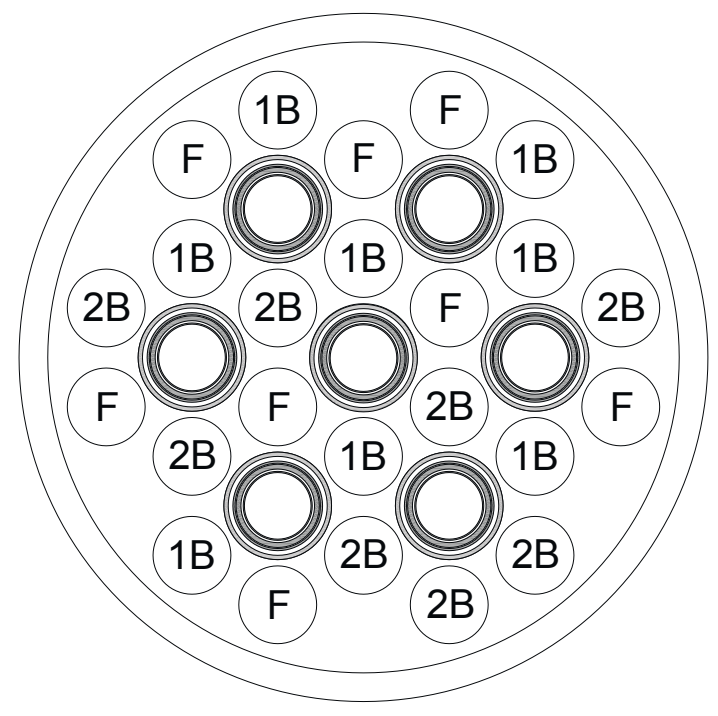

Tilt 1

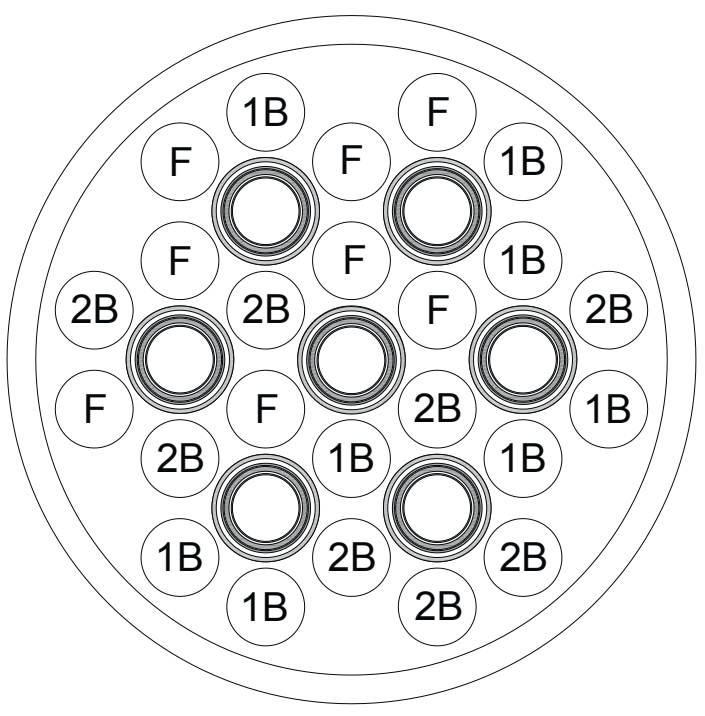

Tilt 3

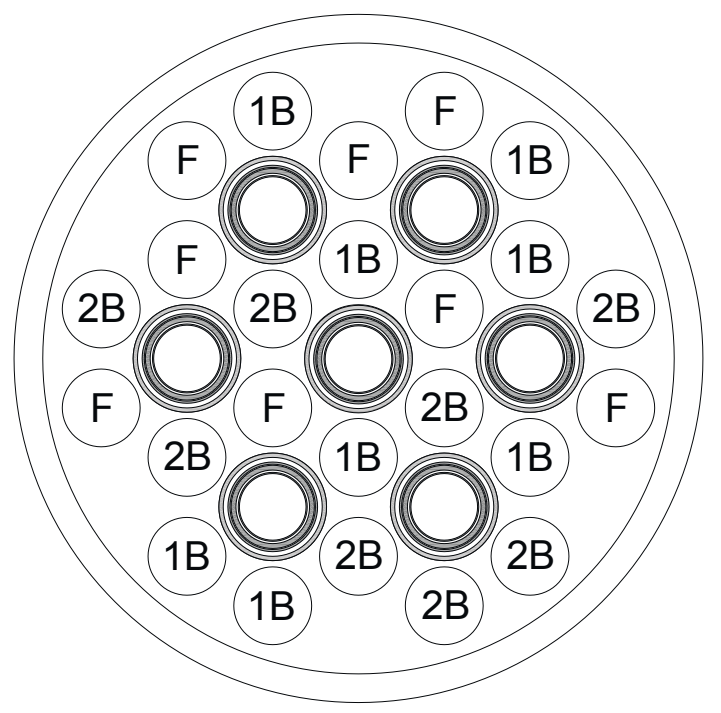

Tilt 2

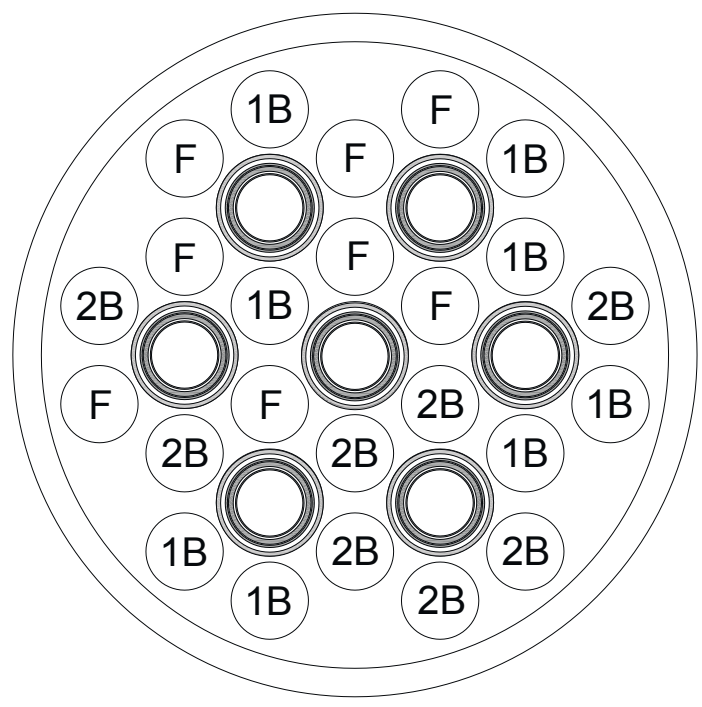

Tilt 4

Figure 9-4. Core loading maps for 3-batch tilted Cylindrical cores. 
Table 9-4. Flux in IPTs and power tilt for prototypic flat loading and tilted cores.

\begin{tabular}{|c|c|c|c|c|}
\hline \multirow{2}{*}{ Core Loading } & \multicolumn{2}{|c|}{$\begin{array}{c}\text { Total Neutron Flux in IPTs } \\
{\left[\mathbf{n} \cdot \mathbf{c m}^{-2} \cdot \mathbf{s}^{-1}\right]}\end{array}$} & \multirow{2}{*}{$\begin{array}{c}\text { Ratio } \\
\text { NW/SE }\end{array}$} & \multirow{2}{*}{$\boldsymbol{k}_{\text {eff }}$} \\
\cline { 2 - 3 } & $\mathbf{N W}$ & $\mathbf{S E}$ & 1.1 & 1.07689 \\
\hline Prototypic Flat & $1.7 \times 10^{15}$ & $1.5 \times 10^{15}$ & 1.5 & 1.07445 \\
\hline Tilt 1 & $1.9 \times 10^{15}$ & $1.3 \times 10^{15}$ & 1.7 & 1.08443 \\
\hline Tilt 2 & $2.0 \times 10^{15}$ & $1.2 \times 10^{15}$ & 1.8 & 1.08781 \\
\hline Tilt 3 & $2.1 \times 10^{15}$ & $1.2 \times 10^{15}$ & 2.0 & 1.09556 \\
\hline Tilt 4 & $2.2 \times 10^{15}$ & $1.1 \times 10^{15}$ & & \\
\hline
\end{tabular}

\subsection{Control Rods}

Exploration of control options was also initiated using the Cylindrical MATRIX core model. In this design, a natural location for shim and/or shutdown rods would be inside the center of some of the fuel assemblies. This is favorable because these would be locations of high rod worth. However, these are also locations of high fast flux that would not be available for irradiation experiments. Using the prototypic core loading discussed above and shown in Figure 9-3, a hollow absorber rod (98\% hafnium and $2 \%$ zirconium, $\rho=13.1739 \mathrm{~g} / \mathrm{cm}^{3}$ ) was conceptualized to fit just inside the inner fuel plate of the assemblies. The composition of this absorber material is that of the ATR neck shim rods. [12] This is shown in Figure 9-5. The rod has Al-6061 cladding and fits inside an Al-6061 guide tube. Unlabeled white portions in the drawing are coolant water. 


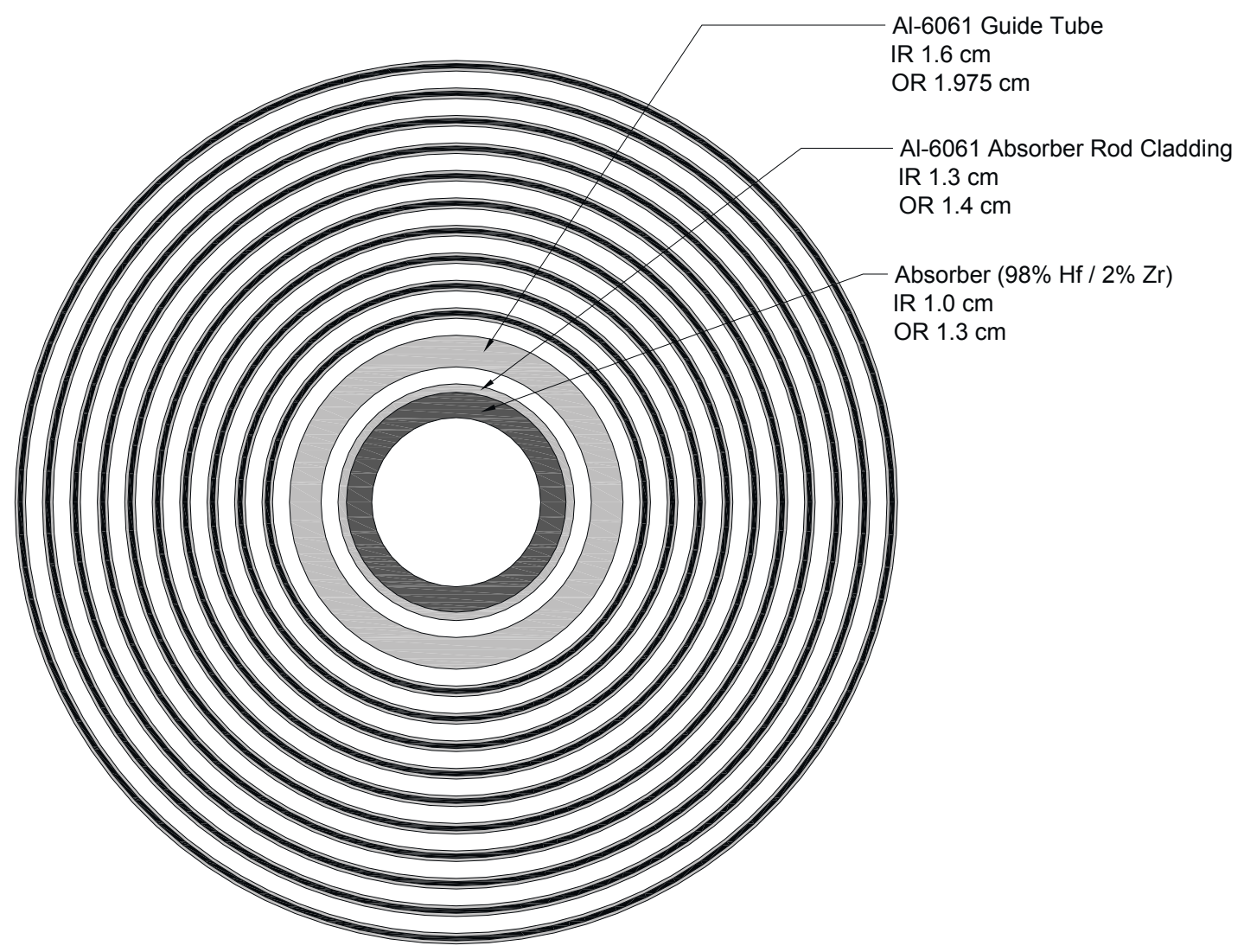

Figure 9-5. Diagram of possible shim rod design inside Cylindrical fuel assembly.

It is desirable to have the worth of a single rod worth be substantial, but less than $\$ 1$ in order to avoid prompt criticality in the event of ejection (if physically possible) or rapid withdrawal. The outer diameter of the hafnium portion of the rods were selected based on this and an approximately $\$ 0.75$ value was targeted.

Table 9-5 shows the worth of absorber rods for various configurations. First, the rods were placed only inside fresh assemblies (8 rods), then inside once-burned assemblies (8 rods). Finally, rods were placed inside both fresh and once-burned assemblies. In all cases, the worth per rod was close to $\$ 0.75$.

Note that the discussion has avoided terming these rods as "shim" or "shut-down". This is because rods such as these, or rods having less absorbing media or dimensions, could be selected for either shutdown or shim.

Rods could be bi-modal wherein they have a gray section to be used as shim followed by a black section for shut-down. As more information is known for the operating parameters of the reactor, these types of concepts may be investigated. 
Table 9-5. Absorber rod worth for various configurations.

\begin{tabular}{|c|c|c|c|c|}
\hline Shim locations & $\begin{array}{c}\text { Number of Rods } \\
\text { Inserted }\end{array}$ & $\boldsymbol{k}_{\text {eff }}$ & $\begin{array}{c}\text { Total Rod } \\
\text { Worth (\$) }^{\mathbf{a}}\end{array}$ & $\begin{array}{c}\text { Worth per } \\
\text { Rod (\$) }\end{array}$ \\
\hline None & 0 & 1.07689 & NA & NA \\
\hline Fresh Fuel only & 8 & 1.03490 & 5.80 & 0.72 \\
\hline 1B Fuel only & 8 & 1.03346 & 6.00 & 0.75 \\
\hline Fresh and 1B Fuel & 16 & 0.99365 & 12.0 & 0.75 \\
\hline
\end{tabular}

\subsection{Potential for Shutdown Rods in Reflector Tank}

In Section 4, the results of the reflector studies indicated that the content of the $\mathrm{D}_{2} \mathrm{O}$ reflector tank has a large reactivity effect. This was demonstrated in the Cylindrical design, but is expected to be true generally due to the similarities of the different MATRIX designs. ${ }^{b}$ For example, as seen in Table 4-1, replacing $\mathrm{D}_{2} \mathrm{O}$ with $\mathrm{H}_{2} \mathrm{O}$ in the reflector tank has a reactivity effect of approximately $-\$ 10$. This signals an opportunity to place shutdown control mechanisms in the reflector tank rather than inside the pressure vessel. The main advantage of this would be to simplify the core internals design and reduce the number of penetrations in the pressure vessel. Shutdown mechanisms in the reflector tank would not have to cross a high-pressure boundary and thus would be simpler and could possibly be less prone to failure. Because so much of the moderation occurs outside the pressure boundary, absorber at the interface between pressure vessel and reflector tank can take advantage of this large re-entering flux.

Shutdown blades in the reflector tank were conceptualized and modeled in the prototypic Cylindrical core described above. Figure 9-6 shows a Serpent rendering of the arrangement of blades surrounding the pressure vessel. The thickness of the blades was $1.5 \mathrm{~cm}$ with $0.7 \mathrm{~cm}$ thick hafnium inside. The clearance between the outer pressure vessel surface and the inner surface of the blades was $0.5 \mathrm{~cm}$. Table $9-6$ contains other relevant parameters of the shutdown blades used for this calculation.

\footnotetext{
${ }^{\mathrm{a}} \beta_{\text {eff }}=0.00650$, approximately the average value during depletion of prototypic cycle.

${ }^{\mathrm{b}}$ The exception to this is likely to be any of the variations calling for a SS304 pressure vessel. The absorption of the stainless steel pressure vessel serves to decouple the core from the reflector tank content.
} 


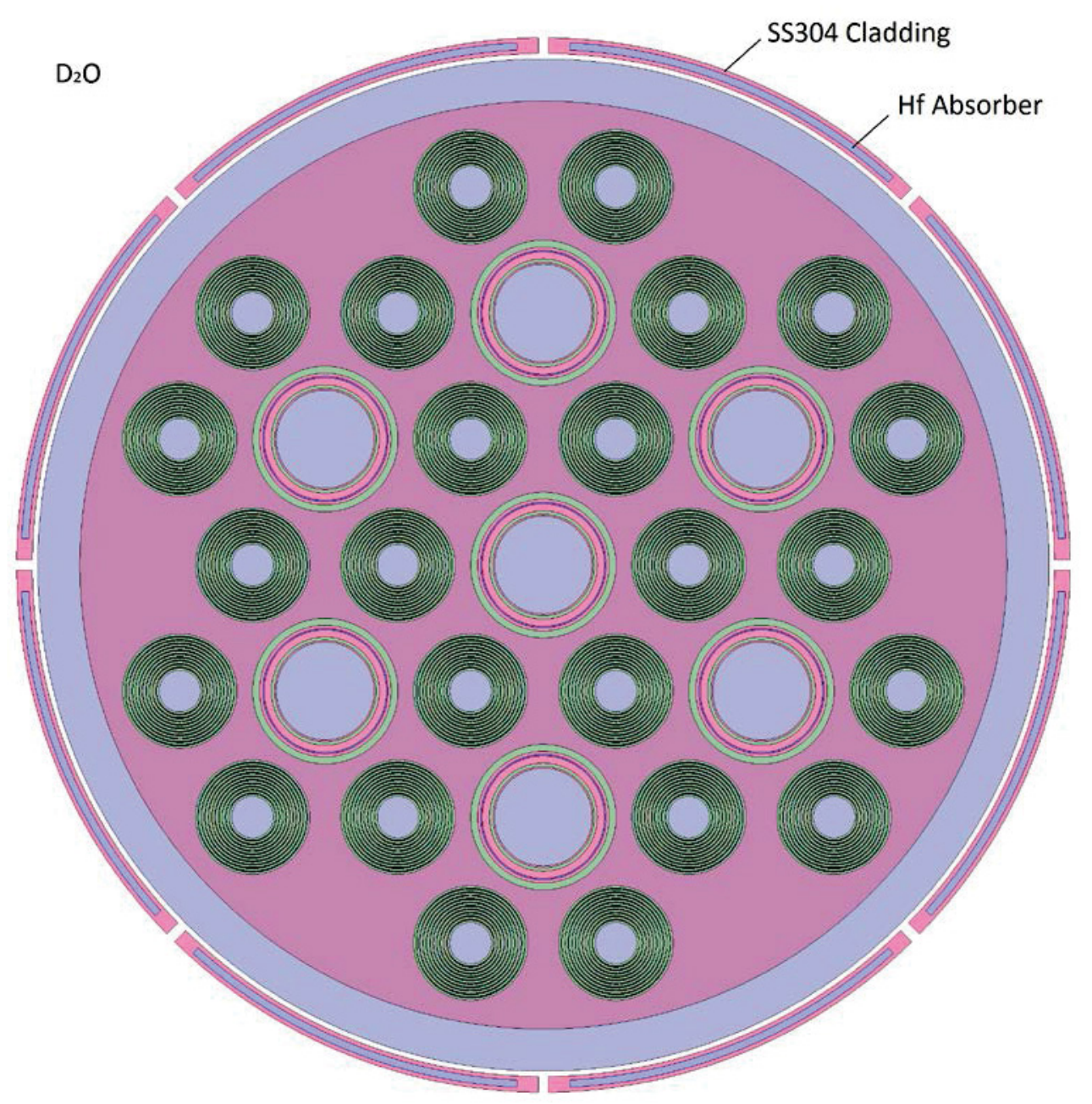

Figure 9-6. View of Cylindrical core with shutdown blades in $\mathrm{D}_{2} \mathrm{O}$ reflector tank.

Table 9-6. Parameters of shutdown blades in reflector tank

\begin{tabular}{|l|l|}
\hline Parameter & Value \\
\hline Blade Thickness $(\mathrm{cm})$ & 1.5 \\
\hline Hafnium Thickness $(\mathrm{cm})$ & 0.7 \\
\hline Exclusion length at end of hafnium inside clad $(\mathrm{cm})$ & 2.0 \\
\hline Clearance between vessel and blade $(\mathrm{cm})$ & 0.5 \\
\hline Distance between ends of blades $(\mathrm{cm})$ & 1.0 \\
\hline Axial height of hafnium $(\mathrm{cm})$ & 140 \\
\hline Axial height of blades $(\mathrm{cm})$ & 144 \\
\hline
\end{tabular}


The value of the inserted control blades described here was approximately $-\$ 15$, resulting in a core multiplication factor of 0.97 . This means that there may be potential for inserting enough reactivity outside the pressure vessel to provide adequate shutdown margin for the core. Recall that this is at BOC with no burnable poisons or shims inserted. With these measures in place, more than adequate shutdown margin may be achievable with a scheme such as this. Various modifications to this concept should be investigated for effectiveness and simplicity.

\subsection{Consideration of Structural Support}

Until this point, cylindrical fuel assemblies have been modeled as only 10 concentric fuel plates surrounding a central filler or test zone. This does not account for 1) the need for structural support in the form of vertical ribs, nor 2) the possibility that it would be more desirable for these fuel assemblies to be three separate arcuate assemblies spanning $120^{\circ}$ each. Reasons for the latter could be fuel management or criticality safety (desire for an inability for a single element to go critical). Though three separate assemblies in each location were not analyzed in this work, Zircaloy ribs were placed into each assembly such that the fuel was divided into three separate arcs, as shown in Figure 9-7. This not only provides some accounting for the loss of fuel meat which would result from these structural components, but gives some estimate of the performance of the alternate three-assembly arrangement.

The resulting reactivity penalty of the addition of the Zircaloy ribs was a reduction in $B_{1}$ from 100 days to 80 days. In a 3 -batch scheme, this amounts to a cycle length reduction from around 50 days to approximately 40 days. This does not compromise the advantage over ATR with regard to cycle length.

Inside the Zircaloy ribs, cadmium wires could be included to serve as burnable absorbers. This arrangement is similar to what is proposed for the LEU fuel in BR-2 [20,21] and is also shown in Figure 9-7. 


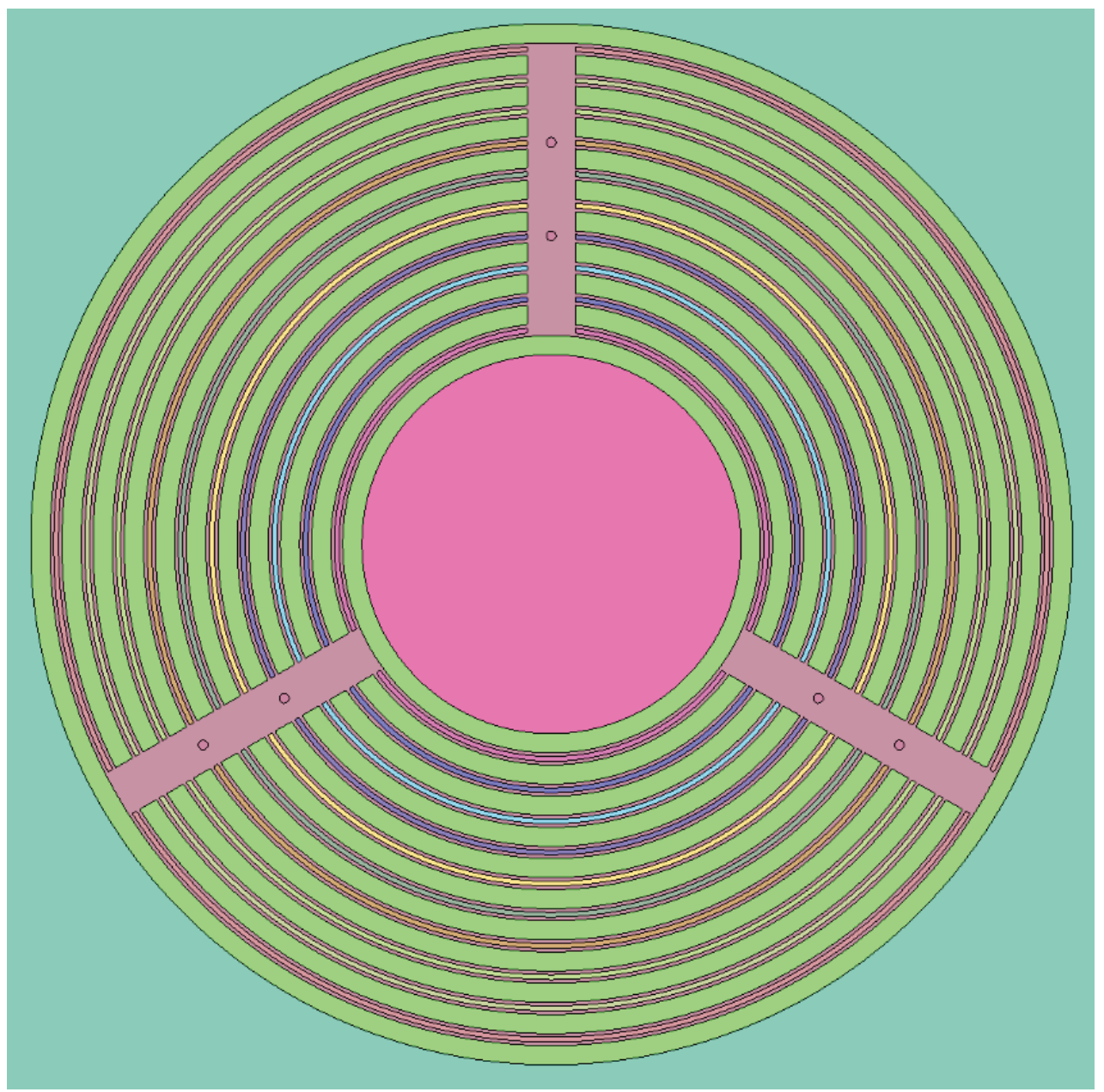

Figure 9-7. Cylindrical fuel element with $0.5 \mathrm{~cm}$ thick side plates and cadmium wires.

\subsection{Test Loop Void Reactivity}

Additional calculations were performed using the sample 3-batch loading from Section Error! Reference source not found. in order to provide a preliminary quantification of the test loop void reactivity. The center IPT was selected for this calculation as it is anticipated to have the most positive void reactivity. In the test space, a range of aluminum/water mixtures was evaluated from $10 \%$ to $40 \%$ water in $10 \%$ increments. For each water content value, a nominal case was modeled in Serpent followed by a voided case, which was modeled by simply removing the water from the IPT test space. The fast/thermal flux ratio for each nominal case was also tallied. Table 9-7 shows the results of the void reactivity and flux ratio calculations. These calculations indicated that in order to keep the void reactivity below $\$ 1$, one would have to limit the water content in the IPT to between 30 and $40 \%$ by volume. This provides a range of fast-tothermal flux ratios from approximately 4 to 2 in this test space. Note that this analysis was performed using coolant water having the same temperature and pressure (and thus density) as the MATRIX cooling water. If the test space contains a specimen being irradiated at PWR conditions, it would then have lower water density. This would allow for more coolant by 
volume to respect the same void reactivity limit as the higher density case evaluated here. One should also note that this calculation was performed using the aluminum rack with no beryllium content. Changes to the rack content should have significant effects on the loop void reactivity and this should be evaluated as the design progresses.

Table 9-7. Void reactivity results for Cylindrical core with prototypic 3-batch loading.

\begin{tabular}{|c|c|c|}
\hline $\begin{array}{c}\text { Nominal } \mathrm{H}_{\mathbf{2}} \mathrm{O} \text { Content } \\
\text { (\% by vol.) }\end{array}$ & $\begin{array}{c}\text { Void Reactivity } \\
\mathbf{( \$ )}\end{array}$ & $\begin{array}{c}\text { Fast/Thermal Flux Ratio } \\
\text { (energy cutoff at } \mathbf{0 . 6 2 5} \mathbf{~ e V})\end{array}$ \\
\hline 10 & 0.32 & 4.3 \\
\hline 20 & 0.54 & 3.0 \\
\hline 30 & 0.86 & 2.2 \\
\hline 40 & 1.08 & 1.6 \\
\hline
\end{tabular}

\section{Conclusions and Path Forward}

This report provides analysis of potential MATRIX reactor concepts that could be deployed in the event the ATR were to shut down permanently. A continuation of FY 2012 work, this report details four main designs, which served as a starting point for work performed in FY 2013, along with a number of variations on these main concepts. Designs were evaluated based on their satisfaction of anticipated customer requirements and the Cylindrical variant was selected for further analysis of options. Because design is at a very early stage, not enough information is known about the concepts for some of the performance metrics to be considered discriminators. Therefore, this downselection should be considered preliminary and the backup alternatives should the other three main designs. Some three-dimensional renderings of the cylindrical MATRIX concept are included in Appendix $\mathrm{C}$ of this report.

The baseline Cylindrical MATRIX design is expected to be capable of higher burnup than the ATR (or longer cycle length given a particular batch scheme). The volume of test space in IPTs is larger in MATRIX with comparable magnitude of neutron flux. In addition to the IPTs, the Cylindrical MATRIX concept features test spaces at the centers of fuel assemblies where very high fast flux can be achieved. This magnitude of fast flux is similar to that achieved in the ATR A-positions, however, the available volume having these conditions is greater in the MATRIX design than in the ATR.

Perhaps the most significant advantage of the Cylindrical MATRIX design over the ATR is the large reflector tank located outside the pressure vessel. Constructed from aluminum and located close to the fuel elements, the low parasitic capture pressure vessel facilitates a very high thermal neutron flux in the reflector tank. This concept is borrowed both from some of the original BATR designs and from the Jules Horowitz reactor under construction in France. Nominally, the reflector tank would be an atmospheric pressure (or near-atmospheric) $\mathrm{D}_{2} \mathrm{O}$ volume to serve as a high thermal flux test space. In this work, an alternative concept was introduced wherein aluminum cans containing $\mathrm{D}_{2} \mathrm{O}$ are placed in an open $\mathrm{H}_{2} \mathrm{O}$ pool. From preliminary neutronic studies, this concept appears to perform well. 
From the analyses performed in this work, it appears that the Cylindrical MATRIX design can be designed to meet the anticipated needs of the ATR replacement reactor. However, this statement must be qualified by acknowledging that this design is quite immature, and therefore any requirements currently met must be re-evaluated as the design matures. Also, some of the requirements were not strictly met, but are believed to be achievable once features to be added later are designed. An example of this is the 3/1 power tilt. This has not been demonstrated in the simulations, but it is expected that a control scheme can be developed that meets this requirement.

As is always the case after a conceptual design study such as this, there is a multitude of follow-on work that needs to be performed in order to verify the feasibility and understand the performance of the MATRIX reactor. This includes, but is not limited to:

- Power Tilt and Shim - The NR requirement of being able to tilt the power at a 3/1 ratio is based on lobe power. Though the concept of lobes may not have explicit meaning in the MATRIX reactor, the ability of achieving a 3/1 ratio of neutron flux in two separate IPTs would adhere to the spirit of this requirement. IN this work, tilt was quantified using the ratio of thermal flux between IPT test spaces. A ratio of $2 / 1$ was achieved with fuel loading alone. The design of shims must be such that not excess reactivity can be held down globally, but in a way that facilitates a sufficient power tilt during depletion.

- Shutdown - Some calculations of rod worth were performed in this work but greater attention should be paid to this feature of the design. The location, geometry, and materials of the shutdown mechanisms should be evaluated in greater detail in future work.

- Secondary Missions - Detailed analysis of performance in specific secondary missions has not been performed. For example, rate of production of various radioisotopes, and the performances of a beam tube or a neutron radiograph should be analyzed. Figures of merit should be not only the performance of each secondary mission individually, but their effects on the core, if any, along with their interference with one another should be considered as well.

- Aluminum Pressure Vessel - The neutronic benefits of an aluminum pressure vessel in the MATRIX are substantial. However, the cost and licensing burdens of using an aluminum pressure vessel over a more conventional material should be analyzed. Frequency of replacement compared to other materials should also be evaluated.

- Fuel Element Hold-Down in Upflow - Hydraulic forces on a fuel element at the flow speeds typical in a high-power reactor such as MATRIX can be greater than the weight of the fuel element. The mechanisms for holding fuel elements in place in upflow should be considered at an early stage. Other items, such as tests and flow restrictors, also must be held in place as their ejection can introduce a positive reactivity effect. This must also be investigated at an early state of analysis.

- Transient Testing - The MATRIX reactor must be capable of producing transient power excursions analogous to those of PALM cycles in the current ATR. A scheme to achieve this should be devised.

- Thermal Hydraulic Analysis - In the early stages of this work, the decision was made to adopt the power density and plate thickness of the ATR for the MATRIX concepts. This 
was done in order to simplify the design space initially, providing some assurance that the steady state thermal hydraulic conditions would be satisfactory. In FY 2012, some thermal hydraulic calculations were performed using RELAP5. This was done, however, with the $100 \mathrm{~cm}$ cores initially conceptualized. These need to be repeated with the 120 $\mathrm{cm}$ tall cores. The potential for passive decay heat removal should be evaluated as well.

\section{Acknowledgements}

The authors would like to acknowledge Mark D. DeHart (INL) for providing a Serpent model of ATR from which the calculations performed in this work were adapted. The authors would also like to thank Paul D. Bayless (INL) for thermal hydraulic support of this project in the previous fiscal year and for contributing the MATRIX acronym. David Combs (INL) developed the 3D renderings of the MATRIX concept shown on the front cover and in Appendix C of this report.

\section{References}

1. Ryskamp, J.M. (ed.), "Safety Requirements, Facility User Needs, and Reactor Concepts for a New Broad Application Test Reactor," Idaho National Laboratory Report, NRRT-N-91-036, October 1991.

2. Terry, W.J. (ed.), "Preliminary Design Studies on the Broad Application Test Reactor," Idaho National Laboratory Report, EGG-NRRT-10350, August 1992.

3. Jahshan, S.N., W.K. Terry, C.D. Fletcher, and G.L. Thinnes, "Parametric Studies on the Broad Application Test Reactor," Idaho National Laboratory Report, EGG-NRE-11017, September 1993.

4. Jahshan, S.N. and W.K. Terry, "A Comparison of Two Versions of a Proposed Test Reactor Using the MCNP Neutronics Code," Nuclear Technology, Vol. 110, pp. 93-105, April 1995.

5. Gougar, H.D. and M.A. Pope, "Requirements, Supporting Technologies, and Recommended Scoping Investigations for a New Test Reactor Fueled with LowEnriched Uranium," Idaho National Laboratory Report, INL/LTD-12-27111, September 2012.

6. Pope, M.A., H. D. Gougar, and J.M. Ryskamp, "Evaluation of Broad Application Test Reactor Concepts with Low-Enriched Uranium Fuel," Idaho National Laboratory, INL/LTD-12-25788, August 2012.

7. Pope, M.A., P.D. Bayless, H. D. Gougar, and J.M. Ryskamp, "Evaluation of Multi-Mission Test Reactor Concepts," Idaho National Laboratory, INL/LTD12-27125, September 2012.

8. A. P. Cochran Letter to A. J. Bieniawski, "Naval Reactors Functional Requirements for the Advanced Test Reactor," NR:RM:APCochran S\#08-04461, Dec 11, 2008. 
9. Leppänen, J. "PSG2/Serpent - A Continuous-energy Monte Carlo Reactor Physics Burnup Calculation Code: User's Manual,” VTT Technical Research Centre of Finland, available at http://montecarlo.vtt.fi, September 5, 2011.

10. Briesmeister, J.F., "MCNP-A General Monte Carlo N-Particle Transport Code," Los Alamos National Laboratory, Los Alamos, New Mexico, 1997.

11. Idaho National Laboratory, "RELAP5-3D ${ }^{\odot}$ Code Manual," INL/EXT-98-00834, Rev. 4.0, June 2012.

12. Idaho National Laboratory, "Advanced Test Reactor: Serpentine Arrangement of Highly Enriched Water-Moderated Uranium-Aluminide Fuel Plates Reflected by Beryllium," HEU-MET-THERM-022, Rev. 4, Available as NEA/NSC/DOC/(95)03/II, September 31 (2010).

13. Email Communication with Patricia Sireta of CEA, Cadarache, April 25, 2012.

14. Cross-section plots generated using KAERI online plotter, available at http://atom.kaeri.re.kr/

15. M. Boyard, J.M. Cherel, C. Pascal, and B. Guigon, "The Jules Horowitz Reactor Core and Cooling System Design," Proceedings of the International Group on Research Reactors (IGORR10), Gaithersburg, Maryland, USA, September 12-16 (2005).

16. Longhurst, G.R. and R.D. Rohe, "Beryllium Use in the Advanced Test Reactor," Proceedings of the $8^{\text {th }}$ IEA International Workshop on Beryllium Technology, December (2007).

17. S.S. Kim, B.G. Schnitzler, “Advanced Test Reactor: Serpentine Arrangement of Highly Enriched Water-Moderated Uranium-Aluminide Fuel Plates Reflected by Beryllium", NEA/NSC/DOC/(95)03/II, Volume II, HEU-MET-THERM-022.

18. Todreas, N.E. and M.S. Kazimi, Nuclear Systems I: Thermal Hydraulic Fundamentals, Hemisphere Publishing Corporation, New York, New York, 1990.

19. S. S. Kim and J. A. McClure, "PDQ Reactor Physics Analysis for the ATR-FSAR Upgrade,” Internal Technical Report PG-T-92-003 Rev. 1, Sept. 1993.

20. Kalcheva, S., G. Van den Branden, and E. Koonen, "Reactivity Performances of Two Prototypes HEU Fuel Elements with Cadmium Wires Irradiated in the BR2 Reactor," Proceedings of RRFM-2012, European Research Reactor Conference, Prague, Czech Republic, March 18-22, 2012.

21. Kalcheva, S., G. and E. Koonen, "Cd's Burnable Poison for BR2 Fuel Element," Proceedings of RRFM-2012, European Research Reactor Conference, Prague, Czech Republic, March 18-22, 2012. 


\section{Appendix A}

\section{Sample Serpent Inputs}




\title{
Cylindrical - Baseline model, single batch depletion
}

\author{
$\%$ title Cylindrical MATRIX core \\ $\%$ Michael Pope \\ $\%$ \\ $\%$ \%

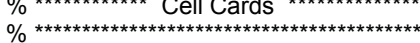 \\ $\%{ }^{* * *}$ Fuel Plates *** Universe $1 * * * *$ \\ cell 11 al6061 $-1 \quad-6061 \%$ \\ $\begin{array}{llllllll}\text { cell } & 2 & 1 & \text { h2o } & 1 & -2 & -60 & 61\end{array} \%$ Water internal to plate 1 \\ $\begin{array}{lllllllll}\text { cell } & 3 & 1 & \text { zirc4 } & 2 & -3 & -60 & 61 & \% \\ & \text { Fuel Clad inner Plate } 1\end{array}$ \\ cell $4 \quad 1$ fuel1 $33-4 \quad-60 \quad 61 \%$ Fuel Meat Plate 1 \\ $\begin{array}{lllllllll}\text { cell } & 5 & 1 & \text { zirc4 } & 4 & -5 & -60 & 61 & \% \\ & \text { Fuel Clad outer Plate } 1\end{array}$

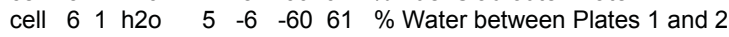

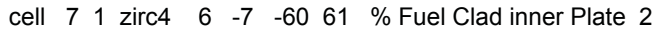 \\ $\begin{array}{llllllllll}\text { cell } & 8 & 1 & \text { fuel2 } & 7 & -8 & -60 & 61 & \% & \text { Fuel Meat Plate } 2\end{array}$ \\ cell 91 zirc4 $\quad 8 \quad-9 \quad-60 \quad 61 \%$ Fuel Clad outer Plate 2

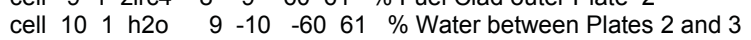 \\ cell 111 zirc4 $10-11 \quad-60 \quad 61 \%$ Fuel Clad inner Plate 3 \\ cell 121 fuel3 $11 \quad-12 \quad-60 \quad 61 \%$ Fuel Meat Plate 3

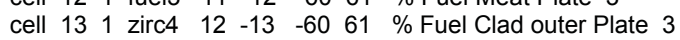 \\ cell $14 \quad 1$ h2o $13-14 \quad-6061 \%$ Water between Plates 3 and 4 \\ cell $15 \quad 1$ zirc4 $14 \quad-15 \quad-60 \quad 61 \quad \%$ Fuel Clad inner Plate 4 \\ cell 161 fuel $4 \quad 15-16 \quad-60 \quad 61 \%$ Fuel Meat Plate 4 \\ cell $17 \quad 1$ zirc4 $16 \quad-17 \quad-60 \quad 61 \%$ Fuel Clad outer Plate 4 \\ cell $18 \quad 1$ h2o $17 \quad-18 \quad-60 \quad 61 \%$ Water between Plates 4 and 5 \\ cell 191 zirc4 $18 \quad-19 \quad-60 \quad 61 \quad \%$ Fuel Clad inner Plate 5 \\ $\begin{array}{llllllll}\text { cell } 20 & 1 & \text { fuel5 } & 19 & -20 & -60 & 61 & \%\end{array}$ Fuel Meat Plate 5 \\ cell $21 \quad 1$ zirc4 $20 \quad-21 \quad-60 \quad 61 \quad \%$ Fuel Clad outer Plate 5 \\ $\begin{array}{lllllll}\text { cell } 22 & 1 & \text { h2o } & 21 & -22 & -60 & 61\end{array} \%$ Water between Plates 5 and 6

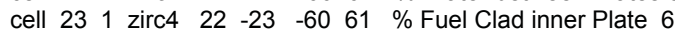

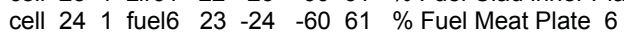

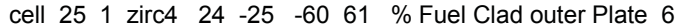

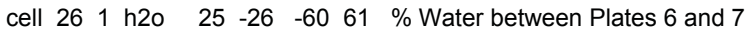 \\ $\begin{array}{lllllllll}\text { cell } 27 & 1 & \text { zirc4 } & 26 & -27 & -60 & 61 & \% & \text { Fuel Clad inner Plate } 7\end{array}$ \\ cell 281 fuel7 $27 \quad-28 \quad-60 \quad 61 \quad \%$ Fuel Meat Plate 7 \\ cell 291 zirc4 $28 \quad-29 \quad-60 \quad 61 \%$ Fuel Clad outer Plate 7 \\ $\begin{array}{lllllllll}\text { cell } 30 & 1 & \text { h2o } & 29 & -30 & -60 & 61 & \% & \text { Water between Plates } 7 \text { and } 8\end{array}$

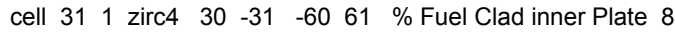 \\ cell 321 fuel8 $31 \quad-32 \quad-60 \quad 61 \%$ Fuel Meat Plate 8

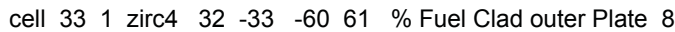 \\ $\begin{array}{lllllllll}\text { cell } 34 & 1 & \text { h2o } & 33 & -34 & -60 & 61 & \%\end{array}$ Water between Plates 8 and 9 \\ $\begin{array}{llllllll}\text { cell } 35 & 1 & \text { zirc4 } & 34 & -35 & -60 & 61 & \% \\ \text { Fuel Clad inner Plate } 9\end{array}$ \\ cell 361 fuel9 $35 \quad-36 \quad-60 \quad 61 \%$ Fuel Meat Plate 9 \\ $\begin{array}{llllllll}\text { cell } 37 & 1 & \text { zirc4 } & 36 & -37 & -60 & 61 & \%\end{array}$

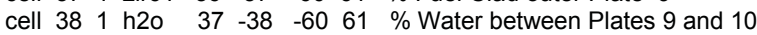 \\ cell 391 zirc4 $38 \quad-39 \quad-60 \quad 61 \%$ Fuel Clad inner Plate 10 \\ cell 401 fuel10 $39 \quad-40 \quad-60 \quad 61 \%$ Fuel Meat Plate 10 \\ cell $41 \quad 1$ zirc4 $40 \quad-41 \quad-60 \quad 61 \%$ Fuel Clad outer Plate 10 \\ cell $42 \quad 1 \quad$ h2o $\quad 41-42 \quad-60 \quad 61 \%$ Water between Plate 10 rack \\ cell 431 AIRack $42 \quad-6061 \%$ al6061 rack
}

$\%$ Upper and lower plates without fuel homogenized

cell 551 TopPlat $-42 \quad 60-62 \%$ upper end plates

cell 561 BotPlat $-42 \quad-6163 \%$ lower end plates

$\%$ Upper and lower end boxes homogenized

cell 571 TopBox $-42 \quad 62-64 \%$ upper end plate

cell 581 BotBox $-42 \quad-63 \quad 65 \%$ lower end plate

$\%$ h2o above and below fuel assembly

cell $59 \quad 1$ h2o $\quad-42 \quad 64 \quad \%$ water above fuel assembly above fuel

$\begin{array}{llllll}\text { cell } 60 & 1 & \mathrm{~h} 2 \mathrm{o} & -42 & -65 & \%\end{array}$

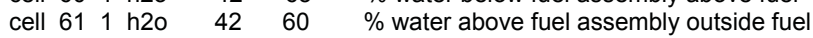

cell $\begin{array}{llllll}62 & 1 & \text { h2o } & 42 & -61 & \%\end{array}$

$\%$ *** Small Test Location *** Universe 2 ****

cell 1202 al6061 $-11061-60 \%$ test space

cell 1212 al6061 $-11060 \quad \%$ above test space

cell 1222 al6061 $-110-61 \%$ below test space

cell 1232 ss304 $110-111 \%$ flow tube

cell 1242 h2o $111-112 \%$ return flow

cell 1252 ss304 $112-113 \%$ pressure tube

cell 1262 helium $113-114 \%$ helium annulus

cell 1272 ss304 $114-115 \%$ insulation jacket

cell 1282 h2o $115-116 \%$ water gap

cell 1292 AIRack $116-117-6061 \%$ baffle

cell 2302 AIRack $117 \quad-60 \quad 61 \%$ al6061 rack adjacent to test location

cell 2282 h2o $116 \quad 60 \quad \%$ water above core next to test loc. 


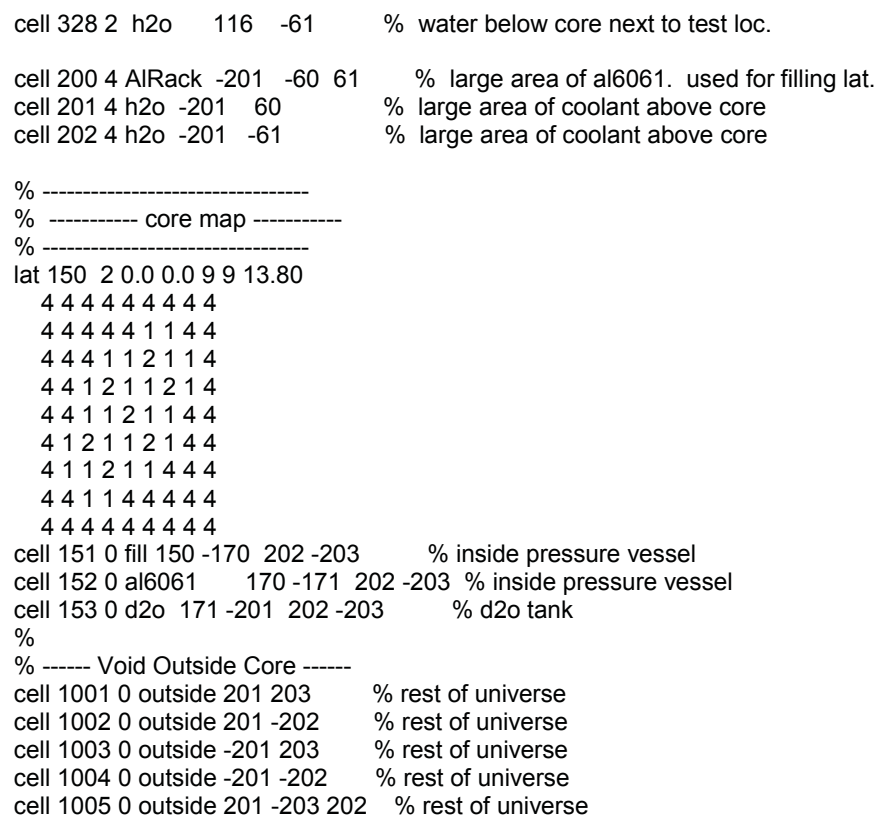

$\%$ Outside of filler

$\%$ Fuel Cld inner Plate 1

$\%$ Fuel Mt inner Plate 1 $\%$ Fuel Mt outer Plate 1

$\%$ Fuel Cld outer Plate 1

$\%$ Fuel Cld inner Plate 2 $\%$ Fuel Mt inner Plate 2

$\%$ Fuel Mt outer Plate 2

$\%$ Fuel Cld outer Plate 2

$\%$ Fuel Cld inner Plate 3

$\%$ Fuel Mt inner Plate 3

$\%$ Fuel Mt outer Plate 3

$\%$ Fuel Cld outer Plate 3

$\%$ Fuel Cld inner Plate 4

$\%$ Fuel Mt inner Plate 4

$\%$ Fuel Mt outer Plate 4

$\%$ Fuel Cld outer Plate 4

$\%$ Fuel Cld inner Plate 5

$\%$ Fuel Mt inner Plate 5

$\%$ Fuel Mt outer Plate 5

$\%$ Fuel Cld outer Plate 5

$\%$ Fuel Cld inner Plate 6

$\%$ Fuel Mt inner Plate 6

$\%$ Fuel Mt outer Plate 6

$\%$ Fuel Cld outer Plate 6

$\%$ Fuel Cld inner Plate 7

$\%$ Fuel Mt inner Plate 7

$\%$ Fuel Mt outer Plate 7

$\%$ Fuel Cld outer Plate 7

$\%$ Fuel Cld inner Plate 8

$\%$ Fuel Mt inner Plate 8

$\%$ Fuel Mt outer Plate 8

$\%$ Fuel Cld outer Plate 8

$\%$ Fuel Cld inner Plate 9

$\%$ Fuel Mt inner Plate 9

$\%$ Fuel Mt outer Plate 9

$\%$ Fuel Cld outer Plate 9

$\%$ Fuel Cld inner Plate 10

$\%$ Fuel Mt inner Plate 10

$\%$ Fuel Mt outer Plate 10

$\%$ Fuel Cld outer Plate 10 


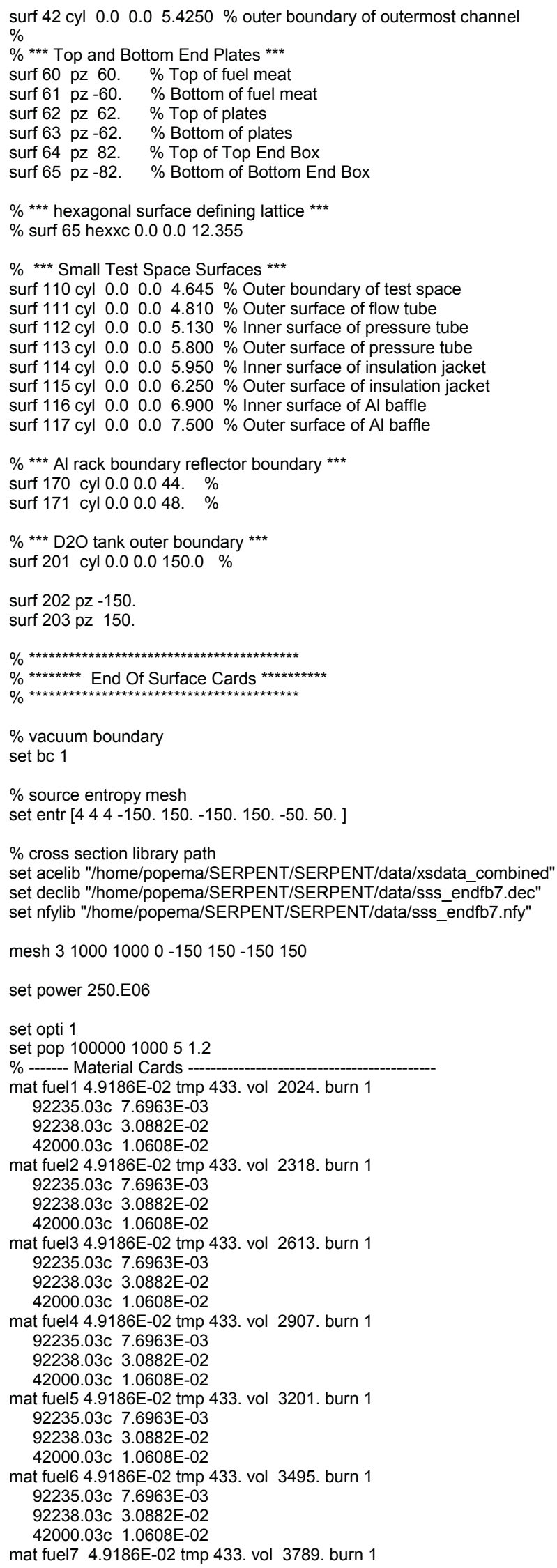


92235.03c 7.6963E-03 92238.03c 3.0882E-02 42000.03c 1.0608E-02

mat fuel8 4.9186E-02 tmp 433. vol 4083. burn 1 92235.03c 7.6963E-03 92238.03c 3.0882E-02 42000.03c 1.0608E-02

mat fuel9 4.9186E-02 tmp 433. vol 4377. burn 1 $92235.03 \mathrm{C}$ 7.6963E-03 $92238.03 \mathrm{c} 3.0882 \mathrm{E}-02$ 42000.03c 1.0608E-02 mat fuel10 4.9186E-02 tmp 433. vol 4671. burn 1 92235.03c 7.6963E-03 92238.03 C 3.0882E-02 42000.03c 1.0608E-02

$\%$----- $\mathrm{H} 2 \mathrm{O}$ Coolant ------

$\% \mathrm{~T}=70 \mathrm{C}, \mathrm{P}=2.3 \mathrm{MPa}$, rho=0.979 $\mathrm{g} / \mathrm{cc}$

mat h2o 9.818E-02 moder h2o 1001 1001.03c 6.545E-02 8016.03c 3.273E-02

$\%$---- D2O

mat d2o 9.986E-02 moder d2o 1002 1002.03c 6.657E-02 8016.03c 3.329E-02 $\%$----- Al-6061 -----

mat al6061 -2.715 \% Al-6061 density $2.715 \mathrm{~g} / \mathrm{cc}$ $14000.03 \mathrm{c}-0.7 \% \quad 0.7 \% \mathrm{Si}$ $26000.03 \mathrm{c}-0.6 \% \quad 0.60 \% \mathrm{Fe}$ 29000.03 c $-0.22 \% \quad 0.22 \% \mathrm{Cu}$ $25055.03 \mathrm{c}-0.08 \% \quad 0.08 \% \mathrm{Mn}$ $12000.03 \mathrm{c}-1.0 \% \quad 1.0 \% \mathrm{Mg}$ $24000.03 c-0.2 \% \quad 0.1 \% \mathrm{Cr}$ $30000.03 \mathrm{c}-0.08 \% \quad 0.08 \% \mathrm{Zn}$ $22000.03 c-0.03 \% \quad 0.03 \% \mathrm{Ti}$ $13027.03 \mathrm{c}-97.09 \% \quad 97.09 \% \mathrm{Al}$

$\%$ assumed $6.3 \%$ by volume $\mathrm{H} 2 \mathrm{O}$

mat be9 1.2188E-01 moder be 4009 moder h2o $1001 \%$ moder be 4009.03c 1.157E-01

1001.03c 4.120E-03

$8016.03 \mathrm{c} 2.060 \mathrm{E}-03$

mat AIBe10 7.09E-02 moder be 4009 moder h2o $1001 \%$ moder be 4009.03c 1.616E-02

13027.03c 4.859E-02

1001.03c 4.120E-03

$8016.03 \mathrm{C}$ 2.060E-03

mat AIBe20 7.85E-02 moder be 4009 moder h2o $1001 \%$ moder be 4009.03c 3.097E-02

13027.03c 4.137E-02

$1001.03 \mathrm{C}$ 4.120E-03

$8016.03 \mathrm{c} 2.060 \mathrm{E}-03$

mat AIBe30 8.55E-02 moder be 4009 moder h2o $1001 \%$ moder be 4009.03c 4.457E-02

13027.03c 3.474E-02

$1001.03 \mathrm{c}$ 4.120E-03

$8016.03 \mathrm{C} 2.060 \mathrm{E}-03$

mat AIBe40 9.19E-02 moder be 4009 moder h2o $1001 \%$ moder be 4009.03c 5.712E-02

13027.03c 2.862E-02

1001.03c 4.120E-03

8016.03c 2.060E-03

mat AlBe60 1.03E-01 moder be 4009 moder h2o $1001 \%$ moder be 4009.03c 7.951E-02

$13027.03 \mathrm{c} 1.771 \mathrm{E}-02$

1001.03c 4.120E-03

8016.03 C 2.060E-03

mat AIBe80 1.13E-01 moder be 4009 moder h2o $1001 \%$ moder be 


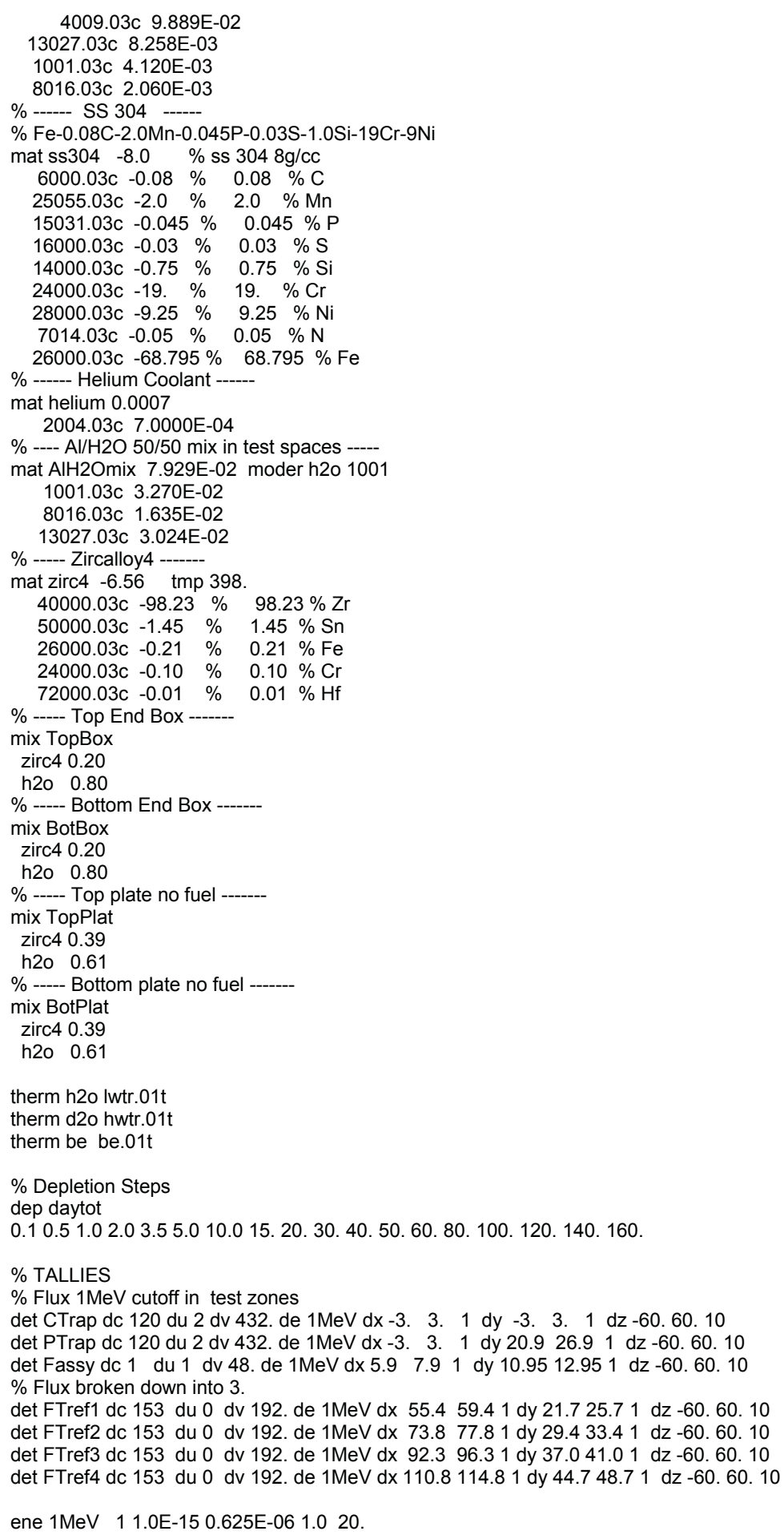




\section{Square - Baseline model, single-batch depletion}

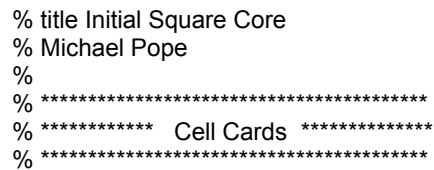


cell $168 \quad 1$ h2o $167 \quad-168 \quad-190 \quad-60 \quad 61$

cell 1691 zirc4 $168 \quad-169-190-60-61$

cell 1701 fuel $169-170-190-6061 \%$ fuel plate 18

cell $171 \quad 1$ zirc4 $170 \quad-171 \quad-190 \quad-60 \quad 61$

cell $172 \quad 1$ h2o $171 \quad-172 \quad-190 \quad-60 \quad 61$

cell $173 \quad 1$ zirc4 $172 \quad-173 \quad-190-60 \quad 61$

cell 1741 fuel $173-174-190-60 \quad 61 \%$ fuel plate 19

cell $175 \quad 1$ zirc4 $174 \quad-175 \quad-190 \quad-60 \quad 61$

cell $176 \quad 1$ h2o $175 \quad-176-190 \quad-6061$

cell $177 \quad 1$ zirc4 $176 \quad-177 \quad-190-60 \quad 61$

cell 1781 fuel $177 \quad-178 \quad-190-60 \quad 61 \%$ fuel plate 20

cell 1791 zirc4 178 -179 $-190-60 \quad 61$

cell $180 \quad 1$ h2o $179 \quad-180 \quad-190 \quad-6061$

cell $181 \quad 1$ zirc4 $180 \quad-181 \quad-190-60 \quad 61$

cell 1821 fuel $181 \quad-182-190-60 \quad 61 \%$ fuel plate 21

cell 1831 zirc4 $182 \quad-183 \quad-190-60 \quad 61$

cell $184 \quad 1$ h2o $183 \quad-190 \quad-60 \quad 61$

\% Upper and lower plates without fuel homogenized

cell 1851 TopPlat $-191 \quad 60-62 \%$ upper end plates

cell 1861 BotPlat $-191 \quad-6163 \%$ lower end plates

$\%$ Upper and lower end boxes homogenized

cell 1871 TopBox $-191 \quad 62-64 \%$ upper end plate

cell 1881 BotBox $-191 \quad-6365 \%$ lower end plate

cell 1891 h2o $-19164 \%$ water above assy

cell 1901 h2o $-191-65 \%$ water below assy

$\%$ *** Test location *** Universe 3 ****

cell 3003 AIRack -191 $61-60$

cell 3013 h2o -19160

cell 3023 h2o $-191-61$

$\%$ *** Control Rod location *** Universe 4 ****

cell 4004 AIRack -191 $61-60$

cell 4014 h2o -19160

cell 4024 h2o $-191-61$

$\%$ *** Rack Fill *** Universe 5 ****

cell 5005 AIRack -191 $61-60$

cell 5015 h2o -19160

cell 5025 h2o $-191-61$

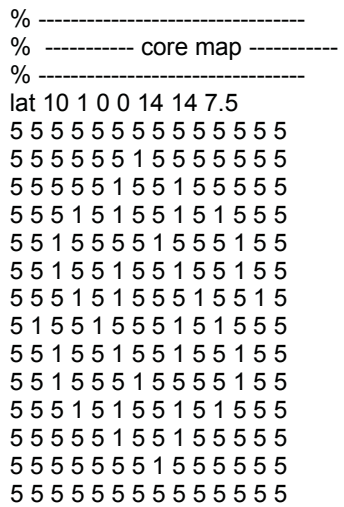

cell 8510 fill $10217227237247257267277-1000802-803 \%$ inside pressure vessel cell 8520 al6061 $1000-1001802-803 \%$ pressure vessel cell 8530 d2o $1001-801802-803 \%$ d2o tank

$\%$ *** Center In-Pile Tube ****

cell 12200 al6061 $-210 \quad 61-60$

cell 12210 al6061 $-210 \quad 60-803$

cell 12220 al6061 $-210-61802$

cell 12230 ss304 $210-211 \quad 802-803$

cell 12240 h2o $211-212 \quad 802-803$

cell 12250 ss304 $212 \quad-213 \quad 802-803$

cell 12260 helium $213-214 \quad 802-803$

cell 12270 ss304 $214 \quad-215 \quad 802-803$

cell 12280 h2o $215-216 \quad 802-803$

cell 12290 AIRack $216-217-60 \quad 61$

cell 12310 h2o $216-217 \quad 60-803$

cell 12320 h2o $216 \quad-217-61802$

$\%$ test space

$\%$ above test space

$\%$ below test space

$\%$ flow tube

$\%$ return flow

$\%$ pressure tube

$\%$ helium annulus

$\%$ insulation jacket

$\%$ water gap

$\%$ baffle - extends to $15 \mathrm{~cm} \times 15 \mathrm{~cm}$ square

$\%$ *** NE In-Pile Tube **** 


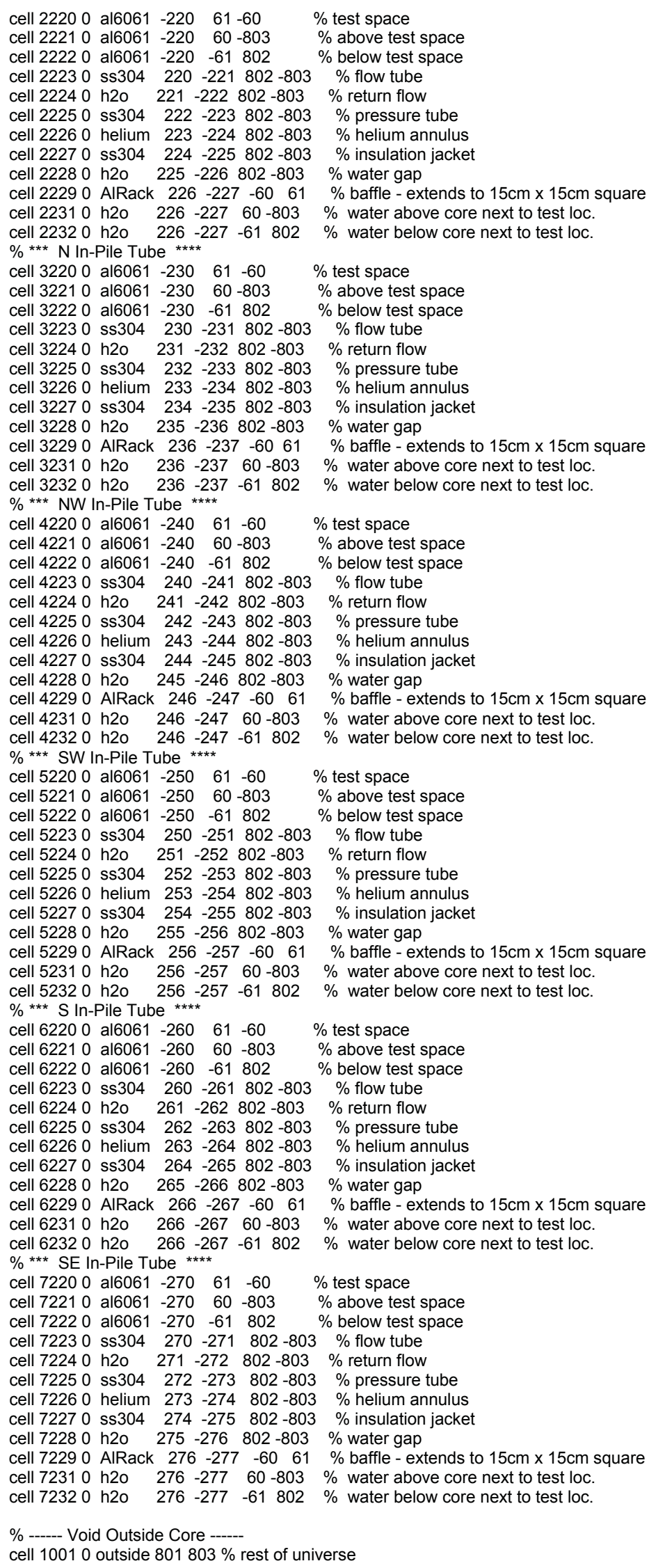


cell 10020 outside $801-802 \%$ rest of universe cell 10030 outside $-801803 \%$ rest of universe cell 10040 outside $-801-802 \%$ rest of universe cell 10050 outside $801-803802 \%$ rest of universe

$\% \% \% \% \% \% \% \% \% \% \% \%$

$\%$ SURFACES

$\% \% \% \% \% \% \% \% \% \% \%$

$\%$ FUEL PLATE SURFACES

surf $100 \mathrm{px}-3.3125$

surf $101 \mathrm{px}-3.2750$

surf $102 \mathrm{px}-3.2250$

surf $103 p x-3.1875$

surf $104 \mathrm{px}-2.9875$

surf $105 \mathrm{px}-2.9500$

surf $106 \mathrm{px}-2.9000$

surf $107 \mathrm{px}-2.8625$

surf $108 \mathrm{px}-2.6625$

surf $109 \mathrm{px}-2.6250$

surf $110 \mathrm{px}-2.5750$

surf $111 \mathrm{px}-2.5375$

surf $112 \mathrm{px}-2.3375$

surf $113 \mathrm{px}-2.3000$

surf $114 \mathrm{px}-2.2500$

surf $115 \mathrm{px}-2.2125$

surf $116 \mathrm{px}-2.0125$

surf $117 \mathrm{px}-1.9750$

surf $118 p x-1.9250$

surf $119 p x-1.8875$

surf $120 p x-1.6875$

surf $121 p x-1.6500$

surf $122 \mathrm{px}-1.6000$

surf $123 \mathrm{px}-1.5625$

surf $124 p x-1.3625$

surf $125 p x-1.3250$

surf $126 \mathrm{px}-1.2750$

surf $127 p x-1.2375$

surf $128 p x-1.0375$

surf $129 \mathrm{px}-1.000$

surf $130 \mathrm{px}-0.9500$

surf $131 p x-0.9125$

surf $132 \mathrm{px}-0.7125$

surf $133 \mathrm{px}-0.6750$

surf $134 p x-0.6250$

surf $135 p x-0.5875$

surf $136 p x-0.3875$

surf $137 p x-0.3500$

surf $138 p x-0.3000$

surf $139 p x-0.2625$

surf $140 \mathrm{px}-0.0625$

surf $141 p x-0.0250$

surf $142 \mathrm{px} 0.0250$

surf $143 \mathrm{px} 0.0625$

surf $144 \mathrm{px} 0.2625$

surf $145 \mathrm{px} 0.3000$

surf $146 \mathrm{px} 0.3500$

surf $147 p \times 0.3875$

surf $148 p \times 0.5875$

surf $149 \mathrm{px} 0.6250$

surf $150 \mathrm{px} 0.6750$

surf $151 p \times 0.7125$

surf $152 \mathrm{px} 0.9125$

surf $153 \mathrm{px} 0.9500$

surf 154 px 1.000

surf 155 px 1.0375

surf $156 \mathrm{px} 1.2375$

surf $157 \mathrm{px} 1.2750$

surf $158 \mathrm{px} 1.3250$

surf $159 \mathrm{px} 1.3625$

surf $160 \mathrm{px} 1.5625$

surf $161 \mathrm{px} 1.6000$

surf 162 px 1.6500

surf 163 px 1.6875

surf 164 px 1.8875

surf $165 \mathrm{px} 1.9250$

surf $166 \mathrm{px} 1.9750$

surf $167 \mathrm{px} 2.0125$

surf $168 \mathrm{px} 2.2125$

surf 169 px 2.2500 
surf $170 \mathrm{px} 2.3000$

surf $171 \mathrm{px} 2.3375$

surf $172 p \times 2.5375$

surf $173 \mathrm{px} 2.5750$

surf $174 \mathrm{px} 2.6250$

surf $175 \mathrm{px} 2.6625$

surf $176 \mathrm{px} 2.8625$

surf $177 \mathrm{px} 2.9000$

surf $178 \mathrm{px} 2.9500$

surf $179 \mathrm{px} 2.9875$

surf $180 \mathrm{px} 3.1875$

surf $181 \mathrm{px} 3.2250$

surf $182 \mathrm{px} 3.2750$

surf $183 \mathrm{px} 3.3125$

surf 190 sqc 003.5125

surf $191 \mathrm{sqc} \quad 0 \quad 0 \quad 3.75$

$\%$ *** Center Space Surfaces ***

surf $210 \mathrm{cyl} \quad 0.0 \quad 0.0 .4 .645 \%$ Outer boundary of test space

surf 211 cyl $0.0 \quad 0.0 \quad 4.810 \%$ Outer surface of flow tube

surf 212 cyl $0.0 \quad 0.0 \quad 5.130 \%$ Inner surface of pressure tube

surf 213 cyl $0.0 \quad 0.0 \quad 5.800 \quad \%$ Outer surface of pressure tube

surf 214 cyl $0.0 \quad 0.0 \quad 5.950 \quad \%$ Inner surface of insulation jacket

$\begin{array}{lllll}\text { surf } 215 \mathrm{cyl} & 0.0 & 0.0 & 6.250 & \%\end{array}$ Outer surface of insulation jacket

surf 216 cyl $0.0 \quad 0.0 \quad 6.900 \quad \%$ Inner surface of Al baffle

surf 217 sqc $0.0 \quad 0.07 .500 \quad \%$ Square Outer surface of Al baffle

$\%$ *** NE Space Surfaces ***

surf 220 cyl 22.5 15. $4.645 \%$ Outer boundary of test space

surf 221 cyl $22.515,4.810 \%$ Outer surface of flow tube

surf 222 cyl 22.5 15. $5.130 \%$ Inner surface of pressure tube

surf 223 cyl 22.5 15. $5.800 \%$ Outer surface of pressure tube

surf 224 cyl 22.5 15. $5.950 \%$ Inner surface of insulation jacket

surf 225 cyl 22.5 15. $6.250 \%$ Outer surface of insulation jacket

surf 226 cyl 22.5 15. $6.900 \%$ Inner surface of Al baffle

surf 227 sqc 22.5 15. $7.500 \%$ Square Outer surface of Al baffle

$\%{ }^{* * *} \mathrm{~N}$ Space Surfaces ***

surf 230 cyl $0.0 \quad 30.0 \quad 4.645 \quad \%$ Outer boundary of test space

surf 231 cyl $0.0 \quad 30.0 \quad 4.810 \%$ Outer surface of flow tube

surf 232 cyl $0.030 .0 \quad 5.130 \quad \%$ Inner surface of pressure tube

surf 233 cyl $0.0 \quad 30.05 .800 \%$ Outer surface of pressure tube

surf 234 cyl $0.0 \quad 30.0 \quad 5.950 \quad \%$ Inner surface of insulation jacket

surf 235 cyl $0.0 \quad 30.0 \quad 6.250 \quad \%$ Outer surface of insulation jacket

surf 236 cyl $0.0 \quad 30.06 .900 \quad \%$ Inner surface of Al baffle

surf 237 sqc $0.0 \quad 30.07 .500 \%$ Square Outer surface of Al baffle $\%$ *** NW Space Surfaces ***

surf $240 \mathrm{cyl}-22.5$ 15. $4.645 \%$ Outer boundary of test space

surf $241 \mathrm{cyl}-22.5$ 15. $4.810 \%$ Outer surface of flow tube

surf 242 cyl -22.5 15. $5.130 \%$ Inner surface of pressure tube

surf $243 \mathrm{cyl}-22.5$ 15. $5.800 \%$ Outer surface of pressure tube

surf $244 \mathrm{cyl}-22.5$ 15. $5.950 \%$ Inner surface of insulation jacket

surf $245 \mathrm{cyl}-22.5$ 15. $6.250 \%$ Outer surface of insulation jacket

surf 246 cyl -22.5 15. $6.900 \%$ Inner surface of Al baffle

surf 247 sqc -22.5 15. $7.500 \%$ Square Outer surface of Al baffle

$\%$ *** SW Space Surfaces ***

surf $250 \mathrm{cyl}-22.5-15$. $4.645 \%$ Outer boundary of test space

surf 251 cyl $-22.5-15.4 .810 \%$ Outer surface of flow tube

surf 252 cyl $-22.5-15.5 .130 \%$ Inner surface of pressure tube

surf 253 cyl $-22.5-15.5 .800 \%$ Outer surface of pressure tube

surf 254 cyl -22.5 -15. $5.950 \%$ Inner surface of insulation jacket

surf $255 \mathrm{cyl}-22.5-15.6 .250 \%$ Outer surface of insulation jacket surf 256 cyl $-22.5-15.6 .900 \%$ Inner surface of Al baffle surf 257 sqc $-22.5-15.7 .500 \%$ Square Outer surface of Al baffle $\% * * *$ S Space Surfaces ***

surf 260 cyl $0.0-30.0 \quad 4.645 \%$ Outer boundary of test space surf 261 cyl $0.0-30.0 \quad 4.810 \%$ Outer surface of flow tube surf 262 cyl $0.0-30.05 .130 \%$ Inner surface of pressure tube surf 263 cyl $0.0-30.0 \quad 5.800 \%$ Outer surface of pressure tube surf 264 cyl $0.0-30.0 \quad 5.950 \%$ Inner surface of insulation jacket surf 265 cyl $0.0-30.0 \quad 6.250 \%$ Outer surface of insulation jacket surf 266 cyl 0.0 -30.0 $6.900 \%$ Inner surface of Al baffle surf 267 sqc $\quad 0.0 \quad-30.0 \quad 7.500 \%$ Square Outer surface of Al baffle $\%$ *** SE Space Surfaces ***

surf 270 cyl $22.5 \quad-15.4 .645 \%$ Outer boundary of test space

surf $271 \mathrm{cyl} 22.5-15.4 .810 \%$ Outer surface of flow tube

surf 272 cyl $22.5-15.5 .130 \%$ Inner surface of pressure tube

surf 273 cyl $22.5-15.5 .800 \%$ Outer surface of pressure tube

surf 274 cyl $22.5-15.5 .950 \%$ Inner surface of insulation jacket

surf 275 cyl $22.5 \quad-15.6 .250 \%$ Outer surface of insulation jacket

surf 276 cyl $22.5 \quad-15$. $6.900 \%$ Inner surface of Al baffle 
surf 277 sqc $22.5-15.7 .500 \%$ Square Outer surface of Al baffle

$\%$ *** Top and Bottom End Plates ***

surf $60 \mathrm{pz} 60$. \% Top of fuel meat

surf $61 \mathrm{pz}-60$. $\%$ Bottom of fuel meat

surf $62 \mathrm{pz} 62$. \% Top of plates

surf $63 \mathrm{pz}-62$. \% Bottom of plates

surf 64 pz 82. \% Top of Top End Box

surf 65 pz -82. \% Bottom of Bottom End Box

$\%{ }^{* * *}$ Pressure Vessel ***

surf $1000 \mathrm{cyl} 0.0 \quad 0.047$

surf $1001 \mathrm{cyl} 0.0 \quad 0.051$

$\%$ infinite surfaces as placeholders for control rod and test locations

surf 300 inf

surf 400 inf

surf 500 inf

$\%$ *** D2O tank outer boundary ***

surf 801 cyl $0.00 .0150 .0 \%$

surf $802 \mathrm{pz}-150$

surf 803 pz 150 .

$\% \% \% \% \% \% \% \% \% \% \% \% \% \% \% \% \%$

$\%$ END OF SURFACES

$\% \% \% \% \% \% \% \% \% \% \% \% \% \% \% \%$

$\%$ vacuum boundary

set bc 1

$\%$ cross section library path !!!! for Fission machine !!!!

set acelib "/home/popema/SERPENT/SERPENT/data/xsdata combined"

set declib "/home/popema/SERPENT/SERPENT/data/sss_endfb7.dec"

set nfylib "/home/popema/SERPENT/SERPENT/data/sss_endfb7.nfy"

mesh $3100010000-150150-150150$

set power 250.E06

set opti 3

set pop 100000100051.2

$\%$----- Material Cards --

$\%$----- $\mathrm{H} 2 \mathrm{O}$ Coolant ---.

$\% \mathrm{~T}=70 \mathrm{C}, \mathrm{P}=2.3 \mathrm{MPa}$, rho $=0.979 \mathrm{~g} / \mathrm{cc}$

mat h2o 9.818E-02 moder h2o 1001

1001.03c 6.545E-02

8016.03c 3.273E-02

$\%$

mat d2o 9.986E-02 moder d2o 1002

1002.03 c 6.657E-02

8016.03c 3.329E-02

$\%$------Fuel-

mat fuel 4.9186E-02 tmp 433. vol 31865. burn 1

92235.03c 7.6963E-03

92238.03 C 3.0882E-02

42000.03c 1.0608E-02

$\%$----- Al-6061 ------

mat al6061 -2.715 \% Al-6061 density $2.715 \mathrm{~g} / \mathrm{cc}$

$14000.03 \mathrm{c}-0.7 \quad \% \quad 0.7 \quad \%$ Si

$26000.03 \mathrm{c}-0.6 \% \quad 0.60 \% \mathrm{Fe}$

29000.03 c $-0.22 \% \quad 0.22 \% \mathrm{Cu}$

$25055.03 \mathrm{c}-0.08 \% \quad 0.08 \% \mathrm{Mn}$

$12000.03 \mathrm{c}-1.0 \% \quad 1.0 \% \mathrm{Mg}$

$24000.03 c-0.2 \% \quad 0.1 \% \mathrm{Cr}$

$30000.03 \mathrm{c}-0.08 \% \quad 0.08 \% \mathrm{Zn}$

$22000.03 \mathrm{c}-0.03 \% \quad 0.03 \% \mathrm{Ti}$

$13027.03 \mathrm{c}-97.09 \% \quad 97.09 \% \mathrm{Al}$

$\%$------ Al-6061 Rack / part water ------

mat AIRack $6.22 \mathrm{E}-02 \%$ \% $\%$ water

14000.03c 3.87E-04\%

26000.03 C 1.67E-04\%

29000.03 C 5.38E-05\%

25055.03 c 2.26E-05\%

12000.03 C $6.39 \mathrm{E}-04 \%$

24000.03 C 5.97E-05\%

30000.03 c 1.90E-05\%

22000.03c 9.73E-06\%

13027.03 C $5.59 \mathrm{E}-02 \%$

1001.03c 3.27E-03 
8016.03c 1.64E-03

$\%$

\% Fe-0.08C-2.0Mn-0.045P-0.03S-1.0Si-19Cr-9Ni

mat ss $304-8.0 \quad \%$ ss $3048 \mathrm{~g} / \mathrm{cc}$

$6000.03 \mathrm{c}-0.08 \% \quad 0.08 \quad \% \mathrm{C}$

$25055.03 \mathrm{c}-2.0 \quad \% \quad 2.0 \quad \% \mathrm{Mn}$

$15031.03 \mathrm{c}-0.045 \% \quad 0.045 \% \mathrm{P}$

$16000.03 c-0.03 \% \quad 0.03 \% \mathrm{~S}$

$14000.03 \mathrm{c}-0.75 \% \quad 0.75 \% \mathrm{Si}$

$24000.03 \mathrm{c}-19 . \quad \% \quad 19 . \% \mathrm{Cr}$

$28000.03 \mathrm{c}-9.25 \% \quad 9.25 \% \mathrm{Ni}$

7014.03c $-0.05 \% \quad 0.05 \% \mathrm{~N}$

$26000.03 \mathrm{c}-68.795 \% \quad 68.795 \% \mathrm{Fe}$

$\%$----- Zircalloy4

mat zirc4 -6.56 tmp 398

$40000.03 c-98.23 \% \quad 98.23 \% \mathrm{Zr}$

$50000.03 \mathrm{c}-1.45 \% \quad 1.45 \% \mathrm{Sn}$

$26000.03 \mathrm{c}-0.21 \% \quad 0.21 \% \mathrm{Fe}$

$24000.03 \mathrm{c}-0.10 \% \quad 0.10 \% \mathrm{Cr}$

$72000.03 \mathrm{c}-0.01 \% \quad 0.01 \% \mathrm{Hf}$

$\%$----- Helium Coolant

mat helium 0.0007

2004.03c 7.0000E-04

\% ----- Top End Box ----

mix TopBox

zirc4 0.20

h2o 0.80

$\%$----- Bottom End Box -----.

mix BotBox

zirc4 0.20

h2o 0.80

$\%$---- Top plate no fuel ------

mix TopPlat

zirc4 0.39

h2o 0.61

$\%$----- Bottom plate no fuel ------.

mix BotPlat

zirc4 0.39

h2o 0.61

$\%$ for installation on Fission

therm h2o Iwtr.01t

therm d2o hwtr.01

therm be be.01t

dep daytot

0.10 .51 .02 .03 .55 .010 .0 15. 20. 30. 40. 50. 60. 80. 100. 120. 140. 160.

$\%$ TALLIES

$\%$ Flux $1 \mathrm{MeV}$ cutoff in test zones

det CTrap dc $1220 \mathrm{du} 0 \mathrm{dv}$ 432. de $1 \mathrm{MeV} d \mathrm{~d}-3$. 3. $1 \mathrm{dy}-3.3 .1 \mathrm{dz}-60.60 .10$

det NTrap dc 3220 du $0 \mathrm{dv} 432$. de $1 \mathrm{MeV} d x-3$. 3. 1 dy 27. 33. $1 \mathrm{dz}-60.60 .10$

det NETrap dc $2220 \mathrm{du} 0 \mathrm{dv}$ 432. de $1 \mathrm{MeV}$ dx 19.525 .51 dy 12. $18.1 \mathrm{dz}-60.60 .10$

det NWTrap dc 4220 du 0 dv 432. de $1 \mathrm{MeV}$ dx $-25.5-19.51$ dy 12. $18.1 \mathrm{dz}-60.60 .10$

$\%$ Flux broken down into 3 .

det FTref1 dc 853 du 0 dv 192. de $1 \mathrm{MeV}$ dx 55.459 .41 dy $21.725 .71 \mathrm{dz}-60.60 .10$ det FTref2 dc 853 du 0 dv 192. de $1 \mathrm{MeV}$ dx 73.877 .81 dy $29.433 .41 \mathrm{dz}-60.60 .10$ det FTref3 dc 853 du 0 dv 192. de $1 \mathrm{MeV}$ dx 92.396 .31 dy $37.041 .01 \mathrm{dz}-60.60 .10$ det FTref4 dc 853 du 0 dv 192. de $1 \mathrm{MeV}$ dx $110.8114 .81 \mathrm{dy} 44.748 .71 \mathrm{dz}-60.60 .10$

ene $1 \mathrm{MeV}$ 11.0E-15 0.625E-06 1.020. 


\title{
Annular-Baseline case, single batch depletion
}

\author{
$\%$ title Baseline Annular Core \\ $\%$ Michael Pope \\ $\%$

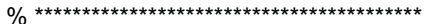

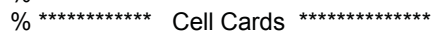

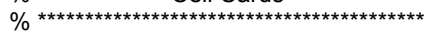 \\ $\%$ *** Universe $1^{* * *}$ Large central Location **** \\ $\%$ *** Test Space **** \\ cell 1401 al6061 $-121 \quad \%$ test space \\ cell 1421 h2o $121-122 \%$ return flow \\ cell 1431 ss304 $122-123 \%$ pressure tube \\ cell 1441 helium $123-124 \%$ helium annulus \\ cell 1451 ss304 $124-125 \%$ insulation jacket \\ cell 1461 h2o $125-126 \quad \%$ water gap \\ cell 1471 al6061 $126-127 \%$ baffle \\ cell $1481 \mathrm{~h} 2 \mathrm{o} \quad 127-1 \quad \%$ water outside baffle inside fuel plate 1 \\ cell 1491 h2o $\quad 48-128-8081 \%$ water outside fuel inside Rack \\ cell 1501 AIRack $128 \quad-80 \quad 81 \%$ al next to fuel \\ cell 1511 h2o $128 \quad 80-203 \quad \%$ water above rack, outside fuel \\ cell 1521 h2o $\quad 128 \quad-81202 \quad \%$ water below rack, outside fuel \\ $\%$ *** NNE Octant *** \\ cell $1002 \quad 1$ zirc4 $\quad 1 \quad-2 \quad-80 \quad 81308302 \%$ Fuel Clad inner Plate 1 \\ cell 10031 fuel $1 \quad 2 \quad-3 \quad-80 \quad 81308302 \%$ Fuel Meat Plate 1 \\ cell $1004 \quad 1$ zirc4 $\quad 3 \quad-4 \quad-80 \quad 81308302 \%$ Fuel Clad outer Plate 1

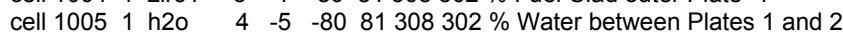 \\ cell 10061 zirc4 $\quad 5 \quad-6 \quad-80 \quad 81308302 \%$ Fuel Clad inner Plate 2 \\ cell 10071 fuel2 $\quad 6 \quad-7 \quad-8081308302 \%$ Fuel Meat Plate 2

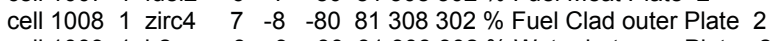 \\ cell 10091 h2o $\quad 8 \quad-9 \quad-8081308302 \%$ Water between Plates 2 and 3 \\ cell 10101 zirc4 $9-10 \quad-80 \quad 81308302 \%$ Fuel Clad inner Plate 3 \\ cell 10111 fuel $3 \quad 10-11 \quad-8081308302 \%$ Fuel Meat Plate 3 \\ cell $1012 \quad 1$ zirc4 $11 \quad-12 \quad-80 \quad 81308302 \%$ Fuel Clad outer Plate 3 \\ cell 10131 h2o $\quad 12-13 \quad-80 \quad 81308302 \%$ Water between Plates 3 and 4 \\ cell 10141 zirc4 $13 \quad-14 \quad-80 \quad 81308302 \%$ Fuel Clad inner Plate 4 \\ cell 10151 fuel $4 \quad 14 \quad-15 \quad-8081308302 \%$ Fuel Meat Plate 4 \\ cell 10161 zirc4 $15 \quad-16 \quad-80 \quad 81308302 \%$ Fuel Clad outer Plate 4 \\ cell $1017 \quad 1$ h2o $\quad 16-17 \quad-80 \quad 81308302 \%$ Water between Plates 4 and 5 \\ cell 10181 zirc4 $17 \quad-18 \quad-80 \quad 81308302 \%$ Fuel Clad inner Plate 5 \\ cell 10191 fuel5 $18-19 \quad-8081308302 \%$ Fuel Meat Plate 5 \\ cell 10201 zirc4 $19 \quad-20 \quad-80 \quad 81308302 \%$ Fuel Clad outer Plate 5 \\ $\begin{array}{llllllll}\text { cell } 1021 & 1 & \text { h2o } & 20 & -21 & -80 & 81308 & 302 \%\end{array}$ Water between Plates 5 and 6 \\ $\begin{array}{lllllllll}\text { cell } 1022 & 1 & \text { zirc4 } & 21 & -22 & -80 & 81308 & 302 & \%\end{array}$ Fuel Clad inner Plate 6 \\ cell 10231 fuel5 $22 \quad-23 \quad-80 \quad 81308302 \%$ Fuel Meat Plate 6 \\ cell $1024 \quad 1$ zirc4 $\quad 23 \quad-24 \quad-80 \quad 81308302 \%$ Fuel Clad outer Plate 6 \\ $\begin{array}{llllllll}\text { cell } 1025 & 1 & \text { h2o } & 24 & -25 & -80 & 81308 & 302 \% \text { Water between Plates } 6 \text { and } 7\end{array}$ \\ $\begin{array}{llllllll}\text { cell } 1026 & 1 & \text { zirc4 } & 25 & -26 & -80 & 81308 & 302\end{array} \%$ Fuel Clad inner Plate 7 \\ cell 10271 fuel7 $\quad 26 \quad-27 \quad-80 \quad 81308302 \%$ Fuel Meat Plate 7 \\ cell $1028 \quad 1$ zirc4 $\quad 27 \quad-28 \quad-80 \quad 81308302 \%$ Fuel Clad outer Plate 7 \\ $\begin{array}{llllllllll}\text { cell } 1029 & 1 & \text { h2o } & 28 & -29 & -80 & 81 & 308 & 302 & \%\end{array}$ Water between Plates 7 and 8 \\ cell $1030 \quad 1$ zirc4 $29 \quad-30 \quad-80 \quad 81308302 \%$ Fuel Clad inner Plate 8 \\ cell $1031 \quad 1$ fuel $8 \quad 30 \quad-31 \quad-80 \quad 81308302 \%$ Fuel Meat Plate 8 \\ cell $1032 \quad 1$ zirc4 $31 \quad-32 \quad-80 \quad 81308302 \%$ Fuel Clad outer Plate 8 \\ cell 10331 h2o $\quad 32-33 \quad-80 \quad 81308302 \%$ Water between Plates 8 and 9 \\ cell $1034 \quad 1$ zirc4 $33 \quad-34 \quad-80 \quad 81308302 \%$ Fuel Clad inner Plate 9 \\ cell 10351 fuel9 $34-35 \quad-80 \quad 81308302 \%$ Fuel Meat Plate 9 \\ $\begin{array}{lllllllll}\text { cell } 1036 & 1 & \text { zirc4 } & 35 & -36 & -80 & 81308 & 302 & \%\end{array}$ Fuel Clad outer Plate 9 \\ cell $1037 \quad 1$ h2o $\quad 36 \quad-37 \quad-8081308302 \%$ Water between Plates 9 and 10 \\ cell 10381 zirc4 $37 \quad-38 \quad-80 \quad 81308302 \%$ Fuel Clad inner Plate 10 \\ cell 10391 fuel10 $38 \quad-39 \quad-80 \quad 81308302 \%$ Fuel Meat Plate 10 \\ cell 10401 zirc4 $39-40 \quad-80 \quad 81308302 \%$ Fuel Clad outer Plate 10 \\ cell $1041 \quad 1$ h2o $\quad 40 \quad-41 \quad-80 \quad 81308302 \%$ Water between Plates 10 and 11 \\ cell $1042 \quad 1$ zirc4 $41-42 \quad-80 \quad 81308302 \%$ Fuel Clad inner Plate 11 \\ cell 10431 fuel11 $42 \quad-43 \quad-80 \quad 81308302 \%$ Fuel Meat Plate 11 \\ cell $1044 \quad 1$ zirc4 $43 \quad-44 \quad-80 \quad 81308302 \%$ Fuel Clad outer Plate 11 \\ cell $1045 \quad 1$ h2o $\quad 44-45 \quad-80 \quad 81308302 \%$ Water between Plates 11 and 12 \\ cell 10461 zirc4 $45 \quad-46 \quad-80 \quad 81308302 \%$ Fuel Clad inner Plate 12 \\ cell 10471 fuel12 $46 \quad-47 \quad-8081308302 \%$ Fuel Meat Plate 12 \\ cell $1048 \quad 1$ zirc4 $\quad 47 \quad-48 \quad-80 \quad 81308302 \%$ Fuel Clad outer Plate 12 \\ $\%$ *** ENE Octant *** \\ cell 11021 zirc4 $1 \quad-2 \quad-80 \quad 81-307305 \%$ Fuel Clad inner Plate 1

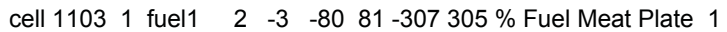

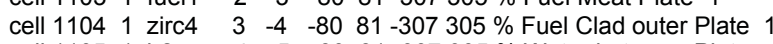 \\ cell 11051 h2o $\quad 4 \quad-5 \quad-80 \quad 81-307305 \%$ Water between Plates 1 and 2 \\ cell $1106 \quad 1$ zirc4 $\quad 5 \quad-6 \quad-80 \quad 81-307305 \%$ Fuel Clad inner Plate 2
}


cell 11071 fuel2 $\quad 6 \quad-7 \quad-80 \quad 81-307305 \%$ Fuel Meat Plate 2

cell 11081 zirc4 $\quad 7 \quad-8 \quad-80 \quad 81-307305 \%$ Fuel Clad outer Plate 2

cell $1109 \quad 1$ h2o $\quad 8 \quad-9 \quad-80 \quad 81-307305 \%$ Water between Plates 2 and 3

cell 11101 zirc4 $\quad 9-10 \quad-80 \quad 81-307305 \%$ Fuel Clad inner Plate 3

cell 11111 fuel3 $10-11 \quad-80 \quad 81-307305 \%$ Fuel Meat Plate 3

cell 11121 zirc4 $11-12 \quad-80 \quad 81-307305 \%$ Fuel Clad outer Plate 3

$\begin{array}{lllllllll}\text { cell } 1113 & 1 & \text { h2o } & 12 & -13 & -80 & 81 & -307 & 305 \%\end{array} \%$ Water between Plates 3 and 4

cell 11141 zirc4 $13-14 \quad-80 \quad 81-307305 \%$ Fuel Clad inner Plate 4

cell 11151 fuel4 $14 \quad-15 \quad-80 \quad 81-307305 \%$ Fuel Meat Plate 4

cell $1116 \quad 1$ zirc4 $15 \quad-16 \quad-80 \quad 81-307305 \%$ Fuel Clad outer Plate 4

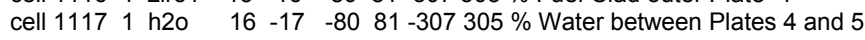

cell 11181 zirc4 $17-18 \quad-80 \quad 81-307305 \%$ Fuel Clad inner Plate 5

cell 11191 fuel5 $18-19 \quad-80 \quad 81-307305 \%$ Fuel Meat Plate 5

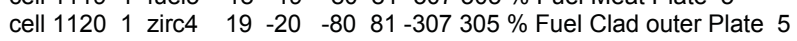

$\begin{array}{lllllllll}\text { cell } 1121 & 1 & \text { h2o } & 20 & -21 & -80 & 81 & -307 & 305 \%\end{array} \%$ Water between Plates 5 and 6

cell 11221 zirc4 $21 \quad-22 \quad-80 \quad 81-307305 \%$ Fuel Clad inner Plate 6

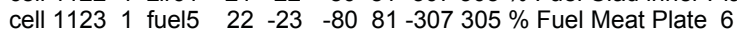

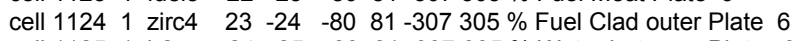

cell 11251 h2o $\quad 24-25 \quad-80 \quad 81-307305 \%$ Water between Plates 6 and 7

cell $1126 \quad 1$ zirc4 $25 \quad-26 \quad-80 \quad 81-307305 \%$ Fuel Clad inner Plate 7

$\begin{array}{llllllllll}\text { cell } 1127 & 1 \text { fuel7 } & 26 & -27 & -80 & 81 & -307 & 305 \% & \text { Fuel Meat Plate } 7\end{array}$

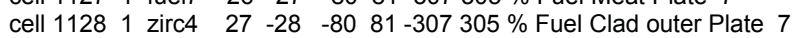

cell $1129 \quad 1$ h2o $\quad 28 \quad-29 \quad-80 \quad 81-307305 \%$ Water between Plates 7 and 8

cell 11301 zirc4 $29-30 \quad-80 \quad 81-307305 \%$ Fuel Clad inner Plate 8

$\begin{array}{llllllllll}\text { cell } 1131 & 1 & \text { fuel } 8 & 30 & -31 & -80 & 81 & -307 & 305 \% & \text { Fuel Meat Plate } 8\end{array}$

cell $1132 \quad 1$ zirc4 $31-32 \quad-80 \quad 81-307305 \%$ Fuel Clad outer Plate 8

cell 11331 h2o $\quad 32-33 \quad-80 \quad 81-307305 \%$ Water between Plates 8 and 9

cell $1134 \quad 1$ zirc4 $33 \quad-34 \quad-80 \quad 81-307305 \%$ Fuel Clad inner Plate 9

cell 11351 fuel9 $34 \quad-35 \quad-80 \quad 81-307305 \%$ Fuel Meat Plate 9

cell $1136 \quad 1$ zirc4 $35 \quad-36 \quad-80 \quad 81-307305 \%$ Fuel Clad outer Plate 9

$\begin{array}{lllllllll}\text { cell } 1137 & 1 & \text { h2o } & 36 & -37 & -80 & 81 & -307 & 305 \% \text { Water between Plates } 9 \text { and } 10\end{array}$

cell $1138 \quad 1$ zirc4 $37 \quad-38 \quad-80 \quad 81-307305 \%$ Fuel Clad inner Plate 10

cell 11391 fuel10 $38 \quad-39 \quad-80 \quad 81-307305 \%$ Fuel Meat Plate 10

cell $1140 \quad 1$ zirc4 $39-40 \quad-80 \quad 81-307305 \%$ Fuel Clad outer Plate 10

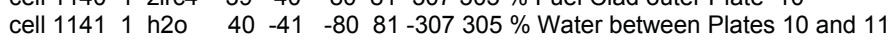

cell $1142 \quad 1$ zirc4 $41-42 \quad-80 \quad 81-307305 \%$ Fuel Clad inner Plate 11

cell 11431 fuel11 $42 \quad-43 \quad-80 \quad 81-307305 \%$ Fuel Meat Plate 11

cell $1144 \quad 1$ zirc4 $43 \quad-44 \quad-80 \quad 81-307305 \%$ Fuel Clad outer Plate 11

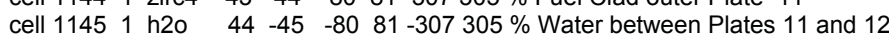

cell 11461 zirc4 $45 \quad-46 \quad-80 \quad 81-307305 \%$ Fuel Clad inner Plate 12

cell 11471 fuel12 $46 \quad-47 \quad-80 \quad 81-307305 \%$ Fuel Meat Plate 12

cell $1148 \quad 1$ zirc4 $47 \quad-48 \quad-80 \quad 81-307305 \%$ Fuel Clad outer Plate 12

$\%{ }^{* * *}$ ESE Octant ***

cell 12021 zirc4

cell 12031 fuel 1

cell 12041 zirc4

cell $12051 \mathrm{~h} 2 \mathrm{o}$

cell 12061 zirc4

cell 12071 fuel2

cell 12081 zirc4

cell $12091 \mathrm{~h} 2 \mathrm{o}$

cell 12101 zirc4

cell 12111 fuel3

cell 12121 zirc4

cell 12131 h2o

cell 12141 zirc 4

cell 12151 fuel 4

cell 12161 zirc4

cell 12171 h2o

cell 12181 zirc 4

cell 12191 fuel5

cell 12201 zirc4

cell $12211 \mathrm{~h} 2 \mathrm{o}$

cell 12221 zirc4

cell 12231 fuel5

cell 12241 zirc4

cell $12251 \mathrm{~h} 2 \mathrm{o}$

cell 12261 zirc4

cell $1227 \quad 1$ fuel 7

cell 12281 zirc 4

cell $12291 \mathrm{~h} 2 \mathrm{o}$

cell $1230 \quad 1$ zirc4

cell 12311 fuel8

cell 12321 zirc4

cell 12331 h2o

cell $1234 \quad 1$ zirc4

cell 12351 fuel9

cell 12361 zirc4

cell 12371 h2o

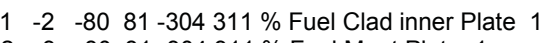

$\begin{array}{lllll}2 & -3 & -80 & 81 & -304 \\ 311 & \% & \text { Fuel Meat Plate } 1\end{array}$

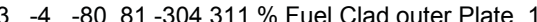

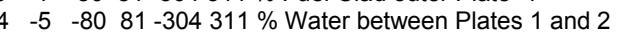

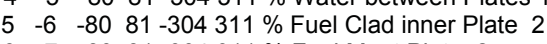

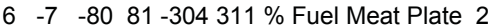

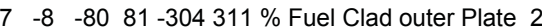

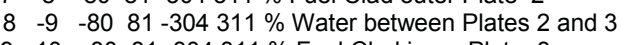

$9 \quad-10 \quad-80 \quad 81-304311 \%$ Fuel Clad inner Plate 3

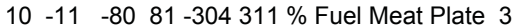

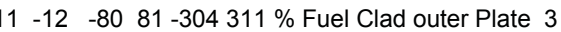

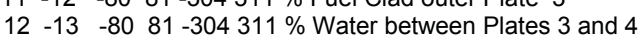

$13-14 \quad-80 \quad 81-304311 \%$ Fuel Clad inner Plate 4

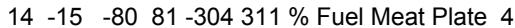

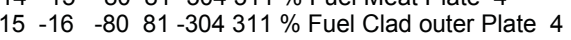

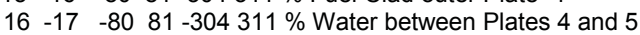

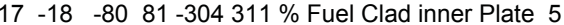

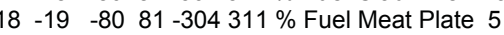

$\begin{array}{lllll}19 & -20 & -80 & 81 & -304 \\ 311 & \% & \text { Fuel Clad outer Plate } 5\end{array}$

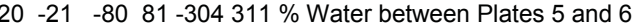

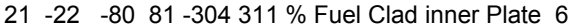

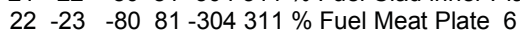

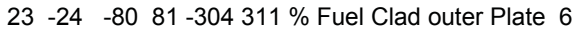

$\begin{array}{lllll}24 & -25 & -80 & 81 & -304 \\ 311 & \%\end{array}$ Water between Plates 6 and 7

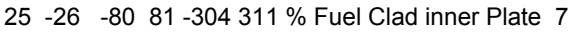

$\begin{array}{lllll}26 & -27 & -80 & 81 & -304 \\ 311 & \% & \text { Fuel Meat Plate } 7\end{array}$

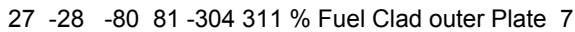

$\begin{array}{llllll}28 & -29 & -80 & 81 & -304 & 311 \%\end{array}$ Water between Plates 7 and 8

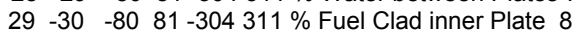

$\begin{array}{lllll}30 & -31 & -80 & 81 & -304 \\ 311 & \% & \text { Fuel Meat Plate } 8\end{array}$

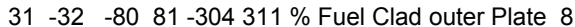

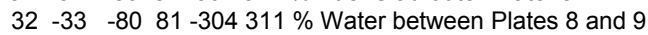

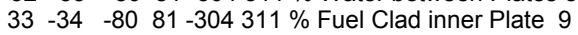

$\begin{array}{lllll}34 & -35 & -80 & 81 & -304 \\ 311 & \% & \text { Fuel Meat Plate } 9\end{array}$

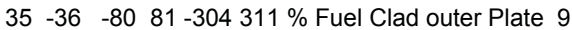

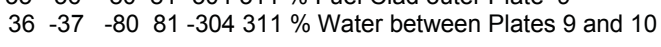


cell 12381 zirc4 $37-38 \quad-80 \quad 81-304311 \%$ Fuel Clad inner Plate 10 cell 12391 fuel10 $38 \quad-39 \quad-80 \quad 81-304311 \%$ Fuel Meat Plate 10

cell $1240 \quad 1$ zirc4 $39 \quad-40 \quad-80 \quad 81-304311 \%$ Fuel Clad outer Plate 10

cell $1241 \quad 1$ h2o $40-41 \quad-80 \quad 81-304311 \%$ Water between Plates 10 and 11

cell $1242 \quad 1$ zirc4 $41 \quad-42 \quad-80 \quad 81-304311 \%$ Fuel Clad inner Plate 11

cell 12431 fuel11 $42 \quad-43 \quad-80 \quad 81-304311 \%$ Fuel Meat Plate 11

cell 12441 zirc4 $43 \quad-44 \quad-80 \quad 81-304311 \%$ Fuel Clad outer Plate 11

cell $1245 \quad 1$ h2o $\quad 44-45 \quad-80 \quad 81-304311 \%$ Water between Plates 11 and 12

cell $1246 \quad 1$ zirc4 $45 \quad-46 \quad-80 \quad 81-304311 \%$ Fuel Clad inner Plate 12

cell 12471 fuel12 $46 \quad-47 \quad-80 \quad 81-304311 \%$ Fuel Meat Plate 12

cell $1248 \quad 1$ zirc4 $47 \quad-48 \quad-80 \quad 81-304311 \%$ Fuel Clad outer Plate 12

$\%$ *** SSE Octant ***

cell 13021 zirc4

cell 13031 fuel 1

cell 13041 zirc4

cell $13051 \mathrm{~h} 2 \mathrm{o}$

cell 13061 zirc

cell 13071 fuel2

cell 13081 zirc

cell $13091 \mathrm{~h} 2 \mathrm{o}$

cell 13101 zirc4

cell 13111 fuel3

cell 13121 zirc4

cell $13131 \mathrm{~h} 2 \mathrm{o}$

cell 13141 zirc

cell 13151 fuel

cell 13161 zirc4

cell $13171 \mathrm{~h} 20$

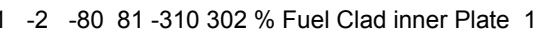

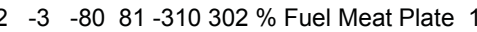

$\begin{array}{llllll}3 & -4 & -80 & 81 & -310 & 302 \%\end{array} \%$ Fuel Clad outer Plate 1

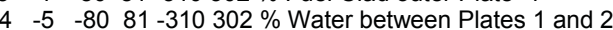

$\begin{array}{llllll}5 & -6 & -80 & 81 & -310 & 302\end{array} \%$ Fuel Clad inner Plate 2

$\begin{array}{llllll}6 & -7 & -80 & 81 & -310 & 302 \%\end{array}$

$\begin{array}{llllll}7 & -8 & -80 & 81 & -310 & 302 \%\end{array} \%$ Fuel Clad outer Plate 2

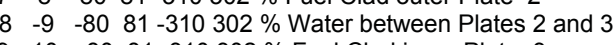

$9 \quad-10 \quad-80 \quad 81-310302 \%$ Fuel Clad inner Plate 3

$10-11-80 \quad 81-310302 \%$ Fuel Meat Plate 3

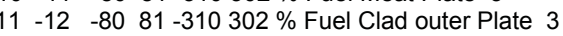

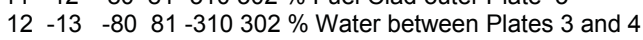

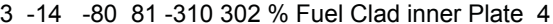

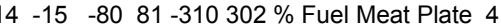

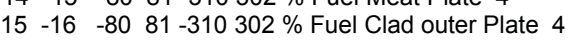

$\begin{array}{llllll}17 & -18 & -80 & 81 & -310 & 302\end{array} \%$ Fuel Clad inner Plate 5

cell 13191 fuel5 $\quad 18 \quad-19 \quad-80 \quad 81-310302 \%$ Fuel Meat Plate 5

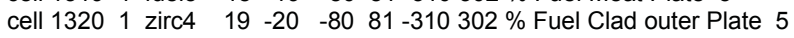

cell $1321 \quad 1$ h2o $\quad 20-21 \quad-80 \quad 81-310302 \%$ Water between Plates 5 and 6

cell $1322 \quad 1$ zirc4 $21 \quad-22 \quad-80 \quad 81-310302 \%$ Fuel Clad inner Plate 6

$\begin{array}{lllllllll}\text { cell } 1323 & 1 & \text { fuel5 } & 22 & -23 & -80 & 81 & -310 & 302\end{array} \%$ Fuel Meat Plate 6

$\begin{array}{llllllllll}\text { cell } 1324 & 1 & \text { zirc4 } & 23 & -24 & -80 & 81 & -310 & 302 & \%\end{array}$ Fuel Clad outer Plate 6

cell 13251 h2o $\quad 24 \quad-25 \quad-80 \quad 81-310302 \%$ Water between Plates 6 and 7

cell $1326 \quad 1$ zirc4 $25 \quad-26 \quad-80 \quad 81-310302 \%$ Fuel Clad inner Plate 7

$\begin{array}{lllllllll}\text { cell } 1327 & 1 & \text { fuel } 7 & 26 & -27 & -80 & 81 & -310 & 302\end{array} \%$ Fuel Meat Plate 7

cell 13281 zirc4 $27 \quad-28 \quad-80 \quad 81-310302 \%$ Fuel Clad outer Plate 7

$\begin{array}{lllllllll}\text { cell } 1329 & 1 & \text { h2o } & 28 & -29 & -80 & 81 & -310 & 302 \%\end{array} \%$ Water between Plates 7 and 8

$\begin{array}{lllllllll}\text { cell } 1330 & 1 & \text { zirc4 } & 29 & -30 & -80 & 81 & -310 & 302\end{array} \%$ Fuel Clad inner Plate 8

cell 13311 fuel8 $30 \quad-31 \quad-80 \quad 81-310302 \%$ Fuel Meat Plate 8

cell $1332 \quad 1$ zirc4 $31 \quad-32 \quad-80 \quad 81-310302 \%$ Fuel Clad outer Plate 8

$\begin{array}{lllllllll}\text { cell } 1333 & 1 & \text { h2o } & 32 & -33 & -80 & 81 & -310 & 302 \% \text { Water between Plates } 8 \text { and } 9\end{array}$

cell 13341 zirc4 $33 \quad-34 \quad-80 \quad 81-310302 \%$ Fuel Clad inner Plate 9

cell 13351 fuel9 $34 \quad-35 \quad-80 \quad 81-310302 \%$ Fuel Meat Plate 9

cell $1336 \quad 1$ zirc4 $35-36 \quad-80 \quad 81-310302 \%$ Fuel Clad outer Plate 9

$\begin{array}{lllllllll}\text { cell } 1337 & 1 & \text { h2o } & 36 & -37 & -80 & 81 & -310 & 302\end{array} \%$ Water between Plates 9 and 10

cell $1338 \quad 1$ zirc4 $37 \quad-38 \quad-80 \quad 81-310302 \%$ Fuel Clad inner Plate 10

cell 13391 fuel10 $38 \quad-39 \quad-80 \quad 81-310302 \%$ Fuel Meat Plate 10

cell 13401 zirc4 $39-40 \quad-80 \quad 81-310302 \%$ Fuel Clad outer Plate 10

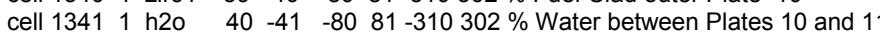

cell $1342 \quad 1$ zirc4 $41 \quad-42 \quad-80 \quad 81-310302 \%$ Fuel Clad inner Plate 11

cell 13431 fuel11 $42 \quad-43 \quad-80 \quad 81-310302 \%$ Fuel Meat Plate 11

cell $1344 \quad 1$ zirc4 $43-44 \quad-80 \quad 81-310302 \%$ Fuel Clad outer Plate 11

cell $1345 \quad 1$ h2o $\quad 44 \quad-45 \quad-80 \quad 81-310302 \%$ Water between Plates 11 and 12

cell $1346 \quad 1$ zirc4 $45 \quad-46 \quad-80 \quad 81-310302 \%$ Fuel Clad inner Plate 12

cell 13471 fuel12 $46 \quad-47 \quad-80 \quad 81-310302 \%$ Fuel Meat Plate 12

cell $1348 \quad 1$ zirc4 $47 \quad-48 \quad-80 \quad 81-310302 \%$ Fuel Clad outer Plate 12

$\%$ *** SSW Octant ***

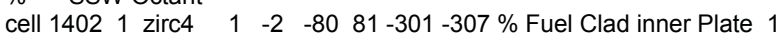

cell 14031 fuel1 $22 \quad-3 \quad-80 \quad 81-301-307 \%$ Fuel Meat Plate 1

cell 14041 zirc4 $\quad 3 \quad-4 \quad-80 \quad 81-301-307 \%$ Fuel Clad outer Plate 1

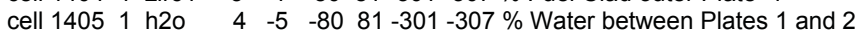

cell 14061 zirc4 $55 \quad-6 \quad-80 \quad 81-301-307 \%$ Fuel Clad inner Plate 2

cell 14071 fuel2 $\quad 6 \quad-7 \quad-80 \quad 81-301-307 \%$ Fuel Meat Plate 2

cell 14081 zirc4 $\quad 7 \quad-8 \quad-80 \quad 81-301-307 \%$ Fuel Clad outer Plate 2

$\begin{array}{llllllllllll}\text { cell } 1409 & 1 & \text { h2o } & 8 & -9 & -80 & 81 & -301 & -307 \% \text { Water between Plates } 2 \text { and } 3\end{array}$

cell 14101 zirc4 $9-10 \quad-80 \quad 81-301-307 \%$ Fuel Clad inner Plate 3

cell 14111 fuel3 $10-11 \quad-80 \quad 81-301-307 \%$ Fuel Meat Plate 3

cell 14121 zirc4 $11 \quad-12 \quad-80 \quad 81-301-307 \%$ Fuel Clad outer Plate 3

cell $1413 \quad 1$ h2o $12 \quad-13 \quad-80 \quad 81-301-307 \%$ Water between Plates 3 and 4

cell 14141 zirc4 $13-14 \quad-80 \quad 81-301-307 \%$ Fuel Clad inner Plate 4

cell 14151 fuel $4 \quad 14 \quad-15 \quad-80 \quad 81-301-307 \%$ Fuel Meat Plate 4

cell 14161 zirc4 $15-16 \quad-80 \quad 81-301-307 \%$ Fuel Clad outer Plate 4

cell $1417 \quad 1$ h2o $\quad 16 \quad-17 \quad-80 \quad 81-301-307 \%$ Water between Plates 4 and 5

cell $1418 \quad 1$ zirc4 $\quad 17 \quad-18 \quad-80 \quad 81-301-307 \%$ Fuel Clad inner Plate 5

$\begin{array}{lllllllll}\text { cell } 1419 & 1 \text { fuel5 } & 18 & -19 & -80 & 81 & -301 & -307 \% \text { Fuel Meat Plate } 5\end{array}$ 
cell 14201 zirc4 $19 \quad-20 \quad-80 \quad 81-301-307 \%$ Fuel Clad outer Plate 5

cell $1421 \quad 1$ h2o $\quad 20-21 \quad-80 \quad 81-301-307 \%$ Water between Plates 5 and 6

cell $1422 \quad 1$ zirc4 $21 \quad-22 \quad-80 \quad 81-301-307 \%$ Fuel Clad inner Plate 6

$\begin{array}{llllllllllll}\text { cell } 1423 & 1 & \text { fuel5 } & 22 & -23 & -80 & 81 & -301 & -307 & \% & \text { Fuel Meat Plate } 6\end{array}$

cell 14241 zirc4 $23-24 \quad-80 \quad 81-301-307 \%$ Fuel Clad outer Plate 6

cell $1425 \quad 1$ h2o $\quad 24-25 \quad-80 \quad 81-301-307 \%$ Water between Plates 6 and 7

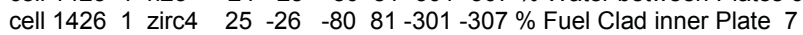

cell 14271 fuel7 $26 \quad-27 \quad-80 \quad 81-301-307 \%$ Fuel Meat Plate 7

cell 14281 zirc4 $27 \quad-28 \quad-80 \quad 81-301-307 \%$ Fuel Clad outer Plate 7

$\begin{array}{llllllllll}\text { cell } 1429 & 1 & \text { h2o } & 28 & -29 & -80 & 81 & -301 & -307 & \%\end{array}$ Water between Plates 7 and 8

$\begin{array}{llllllllll}\text { cell } 1430 & 1 & \text { zirc4 } & 29 & -30 & -80 & 81 & -301 & -307 \% \text { Fuel Clad inner Plate } 8\end{array}$

cell 14311 fuel8 $30 \quad-31 \quad-80 \quad 81-301-307 \%$ Fuel Meat Plate 8

cell $1432 \quad 1$ zirc4 $31-32 \quad-80 \quad 81-301-307 \%$ Fuel Clad outer Plate 8

$\begin{array}{llllllllll}\text { cell } 1433 & 1 & \text { h2o } & 32 & -33 & -80 & 81 & -301 & -307 \% \text { Water between Plates } 8 \text { and } 9\end{array}$

cell 14341 zirc4 $33 \quad-34 \quad-80 \quad 81-301-307 \%$ Fuel Clad inner Plate 9

cell 14351 fuel9 $34-35 \quad-80 \quad 81-301-307 \%$ Fuel Meat Plate 9

cell 14361 zirc4 $35-36 \quad-80 \quad 81-301-307 \%$ Fuel Clad outer Plate 9

$\begin{array}{llllllllll}\text { cell } 1437 & 1 & \text { h2o } & 36 & -37 & -80 & 81 & -301 & -307 \% \text { Water between Plates } 9 \text { and } 10\end{array}$

cell 14381 zirc4 $37 \quad-38 \quad-80 \quad 81-301-307 \%$ Fuel Clad inner Plate 10

cell 14391 fuel10 $38 \quad-39 \quad-80 \quad 81-301-307 \%$ Fuel Meat Plate 10

cell $1440 \quad 1 \quad$ zirc4 $39-40 \quad-80 \quad 81-301-307 \%$ Fuel Clad outer Plate 10

cell $1441 \quad 1$ h2o $\quad 40 \quad-41 \quad-80 \quad 81-301-307 \%$ Water between Plates 10 and 11

cell 14421 zirc4 $41 \quad-42 \quad-80 \quad 81-301-307 \%$ Fuel Clad inner Plate 11

cell 14431 fuel11 $42 \quad-43 \quad-80 \quad 81-301-307 \%$ Fuel Meat Plate 11

cell 14441 zirc4 $43 \quad-44 \quad-80 \quad 81-301-307 \%$ Fuel Clad outer Plate 11

cell 14451 h2o $\quad 44 \quad-45 \quad-80 \quad 81-301-307 \%$ Water between Plates 11 and 12

cell 14461 zirc4 $45 \quad-46 \quad-80 \quad 81-301-307 \%$ Fuel Clad inner Plate 12

cell $1447 \quad 1$ fuel12 $46 \quad-47 \quad-80 \quad 81-301-307 \%$ Fuel Meat Plate 12

cell 14481 zirc4 $47 \quad-48 \quad-80 \quad 81-301-307 \%$ Fuel Clad outer Plate 12

$\%$ *** WSW Octant ***

cell $1502 \quad 1$ zirc4 $1 \quad 1 \quad-2 \quad-80 \quad 81-304308 \%$ Fuel Clad inner Plate 1

cell 15031 fuel1 $\quad 2 \quad-3 \quad-80 \quad 81-304308 \%$ Fuel Meat Plate 1

cell $1504 \quad 1$ zirc4 $\quad 3 \quad-4 \quad-80 \quad 81-304308 \%$ Fuel Clad outer Plate 1

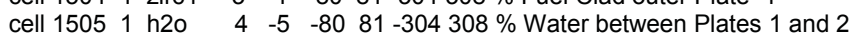

cell 15061 zirc4 $5 \quad 5 \quad-6 \quad-80 \quad 81-304308 \%$ Fuel Clad inner Plate 2

cell 15071 fuel2 $\quad 6 \quad-7 \quad-80 \quad 81-304308 \%$ Fuel Meat Plate 2

$\begin{array}{llllllllll}\text { cell } 1508 & 1 & \text { zirc4 } & 7 & -8 & -80 & 81 & -304 & 308 \% & \text { Fuel Clad outer Plate } 2\end{array}$

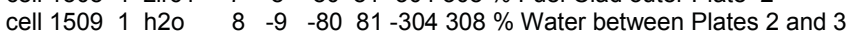

cell 15101 zirc4 $9 \begin{array}{lllll}-10 & -80 & 81 & -304 & 308 \%\end{array} \%$ Fuel Clad inner Plate 3

cell 15111 fuel3 $10-11 \quad-80 \quad 81-304308 \%$ Fuel Meat Plate 3

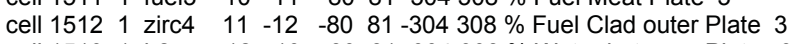

cell $1513 \quad 1$ h2o $\quad 12-13 \quad-80 \quad 81-304308 \%$ Water between Plates 3 and 4

cell $1514 \quad 1$ zirc4 $13-14 \quad-80 \quad 81-304308 \%$ Fuel Clad inner Plate 4

cell 15151 fuel4 $14 \quad-15 \quad-80 \quad 81-304308 \%$ Fuel Meat Plate 4

cell $1516 \quad 1$ zirc4 $15 \quad-16 \quad-80 \quad 81-304308 \%$ Fuel Clad outer Plate 4

cell $1517 \quad 1$ h2o $\quad 16-17 \quad-80 \quad 81-304308 \%$ Water between Plates 4 and 5

cell $1518 \quad 1$ zirc4 $17 \quad-18 \quad-80 \quad 81-304308 \%$ Fuel Clad inner Plate 5

$\begin{array}{llllllllll}\text { cell } 1519 & 1 & \text { fuel5 } & 18 & -19 & -80 & 81 & -304 & 308 \% & \text { Fuel Meat Plate } 5\end{array}$

cell 15201 zirc4 $19-20 \quad-80 \quad 81-304308 \%$ Fuel Clad outer Plate 5

cell $1521 \quad 1$ h2o $\quad 20-21 \quad-80 \quad 81-304308 \%$ Water between Plates 5 and 6

$\begin{array}{lllllllllll}\text { cell } 1522 & 1 & \text { zirc4 } & 21 & -22 & -80 & 81 & -304 & 308 \% & \text { Fuel Clad inner Plate } 6\end{array}$

cell 15231 fuel5 $22 \quad-23 \quad-80 \quad 81-304308 \%$ Fuel Meat Plate 6

cell 15241 zirc4 $23 \quad-24 \quad-80 \quad 81-304308 \%$ Fuel Clad outer Plate 6

cell $1525 \quad 1$ h2o $\quad 24 \quad-25 \quad-80 \quad 81-304308 \%$ Water between Plates 6 and 7

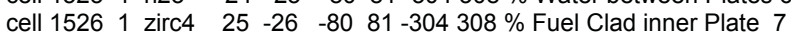

cell 15271 fuel7 $\quad 26 \quad-27 \quad-80 \quad 81-304308 \%$ Fuel Meat Plate 7

cell $1528 \quad 1$ zirc4 $27 \quad-28 \quad-80 \quad 81-304308 \%$ Fuel Clad outer Plate 7

$\begin{array}{llllllllll}\text { cell } 1529 & 1 & \text { h2o } & 28 & -29 & -80 & 81 & -304 & 308 \% & \text { Water between Plates } 7 \text { and } 8\end{array}$

cell 15301 zirc4 $29 \quad-30 \quad-80 \quad 81-304308 \%$ Fuel Clad inner Plate 8

cell 15311 fuel8 $30 \quad-31 \quad-80 \quad 81-304308 \%$ Fuel Meat Plate 8

cell $1532 \quad 1$ zirc4 $31 \quad-32 \quad-80 \quad 81-304 \quad 308 \%$ Fuel Clad outer Plate 8

$\begin{array}{lllllllll}\text { cell } 1533 & 1 & \text { h2o } & 32 & -33 & -80 & 81 & -304 & 308 \% \text { Water between Plates } 8 \text { and } 9\end{array}$

cell $1534 \quad 1$ zirc4 $33 \quad-34 \quad-80 \quad 81-304308 \%$ Fuel Clad inner Plate 9

cell 15351 fuel9 $34 \quad-35 \quad-80 \quad 81-304308 \%$ Fuel Meat Plate 9

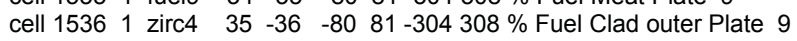

$\begin{array}{llllllllll}\text { cell } 1537 & 1 & \text { h2o } & 36 & -37 & -80 & 81 & -304 & 308 \% & \text { Water between Plates } 9 \text { and } 10\end{array}$

cell 15381 zirc4 $37 \quad-38 \quad-80 \quad 81-304308 \%$ Fuel Clad inner Plate 10

cell 15391 fuel10 $38 \quad-39 \quad-80 \quad 81-304308 \%$ Fuel Meat Plate 10

cell 15401 zirc4 $39-40 \quad-80 \quad 81-304308 \%$ Fuel Clad outer Plate 10

cell 15411 h2o $\quad 40-41 \quad-80 \quad 81-304308 \%$ Water between Plates 10 and 11

cell $1542 \quad 1$ zirc4 $41-42 \quad-80 \quad 81-304308 \%$ Fuel Clad inner Plate 11

cell 15431 fuel11 $42 \quad-43 \quad-80 \quad 81-304308 \%$ Fuel Meat Plate 11

cell 15441 zirc4 $43 \quad-44 \quad-80 \quad 81-304308 \%$ Fuel Clad outer Plate 11

cell $1545 \quad 1$ h2o $\quad 44-45 \quad-80 \quad 81-304308 \%$ Water between Plates 11 and 12

cell $1546 \quad 1$ zirc4 $45 \quad-46 \quad-80 \quad 81-304308 \%$ Fuel Clad inner Plate 12

cell 15471 fuel12 $46 \quad-47 \quad-80 \quad 81-304308 \%$ Fuel Meat Plate 12

cell 15481 zirc4 $47 \quad-48 \quad-80 \quad 81-304308 \%$ Fuel Clad outer Plate 12

$\%{ }^{* * *}$ WNW Octant $* * *$ 
cell 16021 zirc4 cell 16031 fuel 1 cell 16041 zirc cell $16051 \mathrm{~h} 2 \mathrm{o}$ cell 16061 zirc

cell 16071 fuel cell 16081 zirc4 cell 16091 h2o cell 16101 zirc4 cell 16111 fuel3 cell 16121 zirc cell 16131 h2o

cell 16141 zirc4 cell 16151 fuel 4 cell 16161 zirc4 cell 16171 h2o cell 16181 zirc4 cell 16191 fuel5 cell 16201 zirc cell 16211 h2o cell 16221 zirc4 cell 16231 fuel5 cell 16241 zirc 4 cell 16251 h2o cell 16261 zirc4 cell 16271 fuel7 cell 16281 zirc4 cell 16291 h2o cell 16301 zirc4 cell 16311 fuel8 cell 16321 zirc4 cell $16331 \mathrm{~h} 2 \mathrm{o}$ cell 16341 zirc cell 16351 fuelg cell 16361 zirc4 cell 16371 h2o cell 16381 zirc4 cell 16391 fuel

cell 16401 zirc4 cell 16411 h2o cell 16421 zirc4 cell 16431 fuel cell 16441 zirc 4 cell 16451 h2o cell 16461 zirc cell 16471 fuel 12 cell 16481 zirc4

$1-2 \quad-80 \quad 81-310305 \%$ Fuel Clad inner Plate 1 \begin{tabular}{llllll}
2 & -3 & -80 & 81 & -310 & $305 \%$ \\
\hline
\end{tabular}

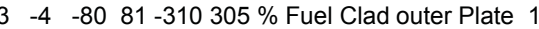

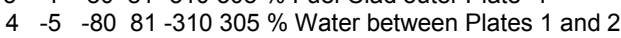

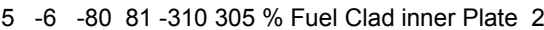
$\begin{array}{llllll}6 & -7 & -80 & 81 & -310 & 305 \%\end{array} \%$ Fuel Meat Plate 2

$\begin{array}{llllll}7 & -8 & -80 & 81 & -310 & 305 \%\end{array} \%$ Fuel Clad outer Plate 2

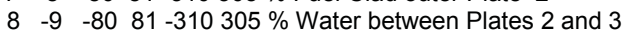
$9 \quad-10 \quad-80 \quad 81-310305 \%$ Fuel Clad inner Plate 3

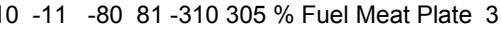

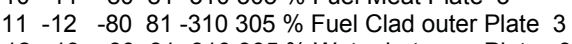

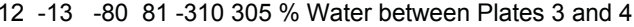

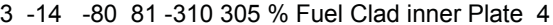

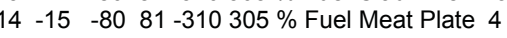

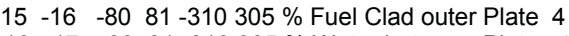

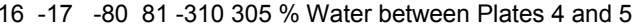

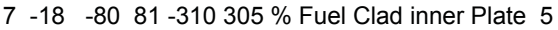

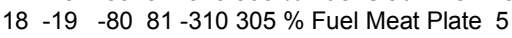

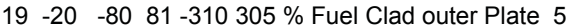
$20-21-80 \quad 81-310305 \%$ Water between Plates 5 and 6

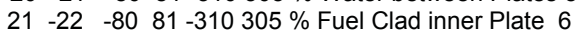

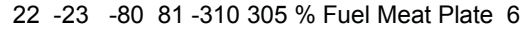

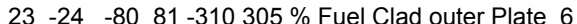

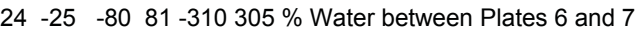

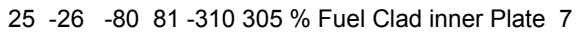

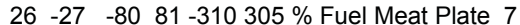

$\begin{array}{llllll}27 & -28 & -80 & 81 & -310 & 305 \%\end{array} \%$ Fuel Clad outer Plate 7

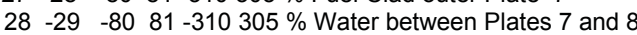

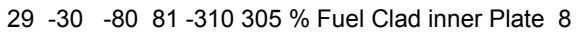

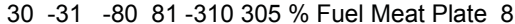

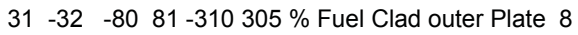

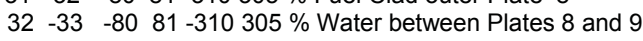

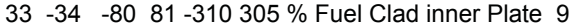

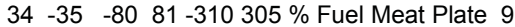

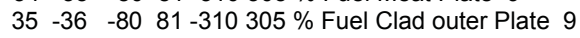

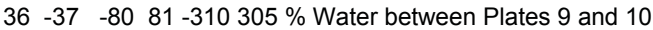

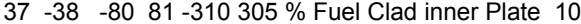

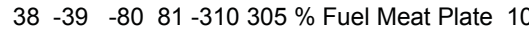

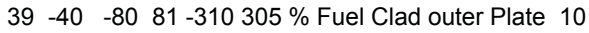

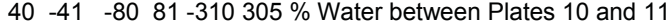

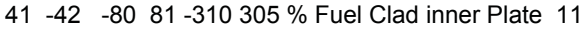

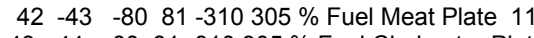

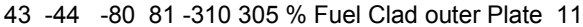

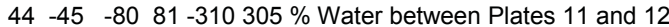

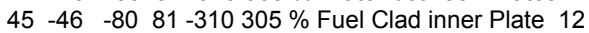
$\begin{array}{llllll}46 & -47 & -80 & 81 & -310 & 305 \%\end{array} \%$ Fuel Meat Plate 12 $\%$ *** NNW Octant *** cell 17021 zirc4 $11-2 \quad-80 \quad 81311-301 \%$ Fuel Clad inner Plate 1 cell 17031 fuel1 $2 \quad-3 \quad-80 \quad 81311-301 \%$ Fuel Meat Plate 1 cell 17041 zirc4 $35-4-8081311-301 \%$ Fuel Clad outer Plate 1 cell 17051 h2o $\quad 4 \quad-5 \quad-80 \quad 81311-301 \%$ Water between Plates 1 and 2 cell 17061 zirc4 5 -6 $-8081311-301 \%$ Fuel Clad inner Plate 2 cell 17071 fuel2 $6 \begin{array}{llllllll}6 & -7 & -80 & 81 & 311-301 \% & \text { Fuel Meat Plate } 2\end{array}$ cell $1708 \quad 1$ zirc4 $7 \quad-8 \quad-80 \quad 81311-301 \%$ Fuel Clad outer Plate 2 cell 17091 h2o $\quad 8 \quad-9-80 \quad 81311-301 \%$ Water between Plates 2 and 3 cell 17101 zirc4 $9-10-80 \quad 81311-301 \%$ Fuel Clad inner Plate 3 cell 17111 fuel3 $10-11-80 \quad 81311-301 \%$ Fuel Meat Plate 3 cell $1712 \quad 1$ zirc4 $11-12 \quad-80 \quad 81311-301 \%$ Fuel Clad outer Plate 3 cell $1713 \quad 1$ h2o $\quad 12-13 \quad-80 \quad 81311-301 \%$ Water between Plates 3 and 4 cell 17141 zirc4 $13-14-8081311-301 \%$ Fuel Clad inner Plate 4 cell 17151 fuel4 $14-15-80 \quad 81311-301 \%$ Fuel Meat Plate 4 cell 17161 zirc4 $15-16-80 \quad 81311-301 \%$ Fuel Clad outer Plate 4 cell $1717 \quad 1$ h2o $\quad 16-17-80 \quad 81311-301 \%$ Water between Plates 4 and 5 cell $1718 \quad 1$ zirc4 $17 \quad-18 \quad-80 \quad 81311-301 \%$ Fuel Clad inner Plate 5 cell 17191 fuel5 $18-19-80 \quad 81311-301 \%$ Fuel Meat Plate 5 cell 17201 zirc4 $19-20 \quad-80 \quad 81311-301 \%$ Fuel Clad outer Plate 5 $\begin{array}{llllllllll}\text { cell } 1721 & 1 \text { h2o } & 20 & -21 & -80 & 81 & 311 & -301 \% \text { Water between Plates } 5 \text { and } 6\end{array}$ cell $1722 \quad 1$ zirc4 $21 \quad-22 \quad-80 \quad 81311-301 \%$ Fuel Clad inner Plate 6 cell 17231 fuel5 $22 \quad-23-80 \quad 81311-301 \%$ Fuel Meat Plate 6 cell $1724 \quad 1$ zirc4 $23 \quad-24 \quad-80 \quad 81311-301 \%$ Fuel Clad outer Plate 6 $\begin{array}{lllllllll}\text { cell } 1725 & 1 & \text { h2o } & 24 & -25 & -80 & 81 & 311 & -301 \% \text { Water between Plates } 6 \text { and } 7\end{array}$ cell $1726 \quad 1$ zirc4 $25 \quad-26 \quad-80 \quad 81311-301 \%$ Fuel Clad inner Plate 7 cell 17271 fuel7 $26 \quad-27 \quad-80 \quad 81311-301 \%$ Fuel Meat Plate 7 cell $1728 \quad 1$ zirc4 $27 \quad-28 \quad-80 \quad 81311-301 \%$ Fuel Clad outer Plate 7 $\begin{array}{lllllllllll}\text { cell } 1729 & 1 & \text { h2o } & 28 & -29 & -80 & 81 & 311 & -301 \% \text { Water between Plates } 7 \text { and } 8\end{array}$ cell 17301 zirc4 $29-30-80 \quad 81311-301 \%$ Fuel Clad inner Plate 8 cell 17311 fuel8 $30 \quad-31 \quad-80 \quad 81311-301 \%$ Fuel Meat Plate 8 cell $1732 \quad 1$ zirc4 $31 \quad-32 \quad-80 \quad 81311-301 \%$ Fuel Clad outer Plate 8 
cell 17331 h2o $32-33-80 \quad 81311-301 \%$ Water between Plates 8 and 9 cell 17341 zirc4 $33 \quad-34-80 \quad 81311-301 \%$ Fuel Clad inner Plate 9

cell 17351 fuel9 $34-35-80 \quad 81311-301 \%$ Fuel Meat Plate 9

$\begin{array}{lllllllllll}\text { cell } 1736 & 1 & \text { zirc4 } & 35 & -36 & -80 & 81 & 311 & -301 \% \text { Fuel Clad outer Plate } 9\end{array}$

cell $1737 \quad 1$ h2o $\quad 36-37 \quad-80 \quad 81311-301 \%$ Water between Plates 9 and 10

cell 17381 zirc4 $37-38-80 \quad 81311-301 \%$ Fuel Clad inner Plate 10

cell 17391 fuel10 $38 \quad-39-80 \quad 81311-301 \%$ Fuel Meat Plate 10

cell 17401 zirc4 $39-40 \quad-80 \quad 81311-301 \%$ Fuel Clad outer Plate 10

cell $1741 \quad 1$ h2o $\quad 40 \quad-41-80 \quad 81311-301 \%$ Water between Plates 10 and 11

cell 17421 zirc4 $41-42-8081311-301 \%$ Fuel Clad inner Plate 11

cell 17431 fuel11 $42 \quad-43 \quad-80 \quad 81311-301 \%$ Fuel Meat Plate 11

cell $1744 \quad 1$ zirc4 $43 \quad-44 \quad-80 \quad 81311-301 \%$ Fuel Clad outer Plate 11

cell $1745 \quad 1$ h2o $\quad 44-45 \quad-80 \quad 81311-301 \%$ Water between Plates 11 and 12

cell 17461 zirc4 $45 \quad-46-80 \quad 81311-301 \%$ Fuel Clad inner Plate 12

cell 17471 fuel12 $46 \quad-47-80 \quad 81311-301 \%$ Fuel Meat Plate 12

cell 17481 zirc4 $47 \quad-48$-80 $81311-301 \%$ Fuel Clad outer Plate 12

$\%$ Other materials above and below fuel assembly

cell $\begin{array}{lllllll}77 & 1 & \mathrm{~h} 2 \mathrm{o} & 1 & -128 & 64 & -203\end{array} \%$ water above fuel assembly

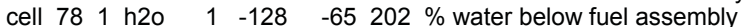

$\%$ Upper and lower plates without fuel homogenized

cell 1851 TopPlat $1 \quad-128 \quad 80-62 \%$ upper end plates

cell 1861 BotPlat $1-128-8163 \%$ lower end plates

$\%$ Upper and lower end boxes homogenized

cell $187 \quad 1$ TopBox $1-128 \quad 62-64 \%$ upper end box

cell 1881 BotBox $\quad 1 \quad-128 \quad-63 \quad 65 \%$ lower end box

$\%$ Side plates (fused adjacent plates

cell 901 zirc4 1 -48 $301-302 \quad 303-80 \quad 81 \%$ N sideplates

cell 911 zirc4 1 -48 $307-308 \quad 303-80 \quad 81 \%$ NE sideplates

cell 921 zirc4 $1-48 \quad 304-305 \quad 300-80 \quad 81 \%$ E sideplates

cell 931 zirc4 $1-48 \quad 310-311-303-80 \quad 81 \%$ SE sideplates

cell 941 zirc4 1 -48 $301-302-303-80 \quad 81 \%$ S sideplates

cell 951 zirc4 1 -48 $307-308-303-80 \quad 81 \%$ SW sideplates

cell 961 zirc4 $1-48 \quad 304-305-300-80 \quad 81 \%$ W sideplates

cell 971 zirc4 1 -48 $310-311303-80 \quad 81 \%$ NW sideplates

$\%$ *** Outer Test Location *** Universe 2 ****

cell 1202 al6061 $-220 \quad \%$ test space

cell 1212 ss304 220 -221 \% flow tube

cell 1222 h2o $221-222 \%$ return flow

cell 1232 ss304 $222-223 \%$ pressure tube

cell 1242 helium $223-224 \%$ helium annulus

cell 1252 ss304 $224-225 \%$ insulation jacket

cell 1262 h2o $225-226 \%$ water gap

cell 1272 al6061 $226-227 \%$ guide tube

cell 1282 h2o $227-228 \quad \%$ water

cell 1292 al6061 $228-229 \%$ safety rod follower

cell 1302 h2o $229-126 \%$ water just inside baffle

cell 1312 al6061 $126-127 \%$ baffle

cell 1322 h2o $127 \quad-1 \quad \%$ water just inside fuel plate 1

cell 1332 h2o $48-128-8081 \%$ water outside fuel inside rack cell 1342 AIRack $128 \quad-8081 \%$ Al Rack next to fuel

cell 1352 h2o $\quad 128 \quad 80-203 \%$ water above rack, outside fuel

$\begin{array}{lllll}\text { cell } 1362 \text { h2o } & 128 & -81 & 202 \% & \% \text { water below rack, outside fuel }\end{array}$

$\%$ *** NNE Octant ***

cell 20022 zirc4 $\quad 1 \quad-2 \quad-8081308302 \%$ Fuel Clad inner Plate 1

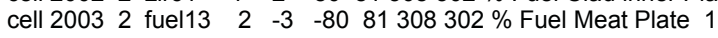

cell 20042 zirc4 $\quad 3 \quad-4 \quad-80 \quad 81308302 \%$ Fuel Clad outer Plate 1

cell 20052 h2o $\quad 4 \quad-5 \quad-8081308302 \%$ Water between Plates 1 and 2

cell 20062 zirc4 $\quad 5 \quad-6 \quad-80 \quad 81308302 \%$ Fuel Clad inner Plate 2

cell 20072 fuel14 $6 \begin{array}{llllll}6 & -7 & -80 & 81308 & 302 \% & \text { Fuel Meat Plate } 2\end{array}$

cell $2008 \quad 2$ zirc4 $\quad 7 \quad-8 \quad-80 \quad 81308302 \%$ Fuel Clad outer Plate 2

cell 20092 h2o $\quad 8 \quad-9 \quad-80 \quad 81308302 \%$ Water between Plates 2 and 3

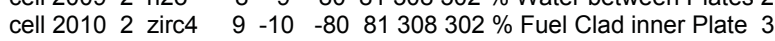

cell 20112 fuel15 $10-11 \quad-80 \quad 81308302 \%$ Fuel Meat Plate 3

cell $2012 \quad 2$ zirc4 $11 \quad-12 \quad-80 \quad 81308302 \%$ Fuel Clad outer Plate 3

cell 20132 h2o $\quad 12-13 \quad-80 \quad 81308302 \%$ Water between Plates 3 and 4

cell 20142 zirc4 $13 \quad-14 \quad-80 \quad 81308302 \%$ Fuel Clad inner Plate 4

cell 20152 fuel16 $14 \quad-15 \quad-80 \quad 81308302 \%$ Fuel Meat Plate 4

cell 20162 zirc4 $15 \quad-16 \quad-80 \quad 81308302 \%$ Fuel Clad outer Plate 4

$\begin{array}{llllllll}\text { cell } 2017 & 2 & \text { h2o } & 16 & -17 & -80 & 81308 & 302 \% \text { Water between Plates } 4 \text { and } 5\end{array}$

cell 20182 zirc4 $17 \quad-18 \quad-80 \quad 81308302 \%$ Fuel Clad inner Plate 5

cell 20192 fuel17 $18 \quad-19 \quad-80 \quad 81308302 \%$ Fuel Meat Plate 5

cell 20202 zirc4 $19 \quad-20 \quad-80 \quad 81308302 \%$ Fuel Clad outer Plate 5

$\begin{array}{llllllllll}\text { cell } 2021 & 2 & \text { h2o } & 20 & -21 & -80 & 81 & 308 & 302 & \%\end{array}$ Water between Plates 5 and 6

cell $2022 \quad 2$ zirc4 $21 \quad-22 \quad-80 \quad 81308302 \%$ Fuel Clad inner Plate 6

cell 20232 fuel18 $22 \quad-23 \quad-80 \quad 81308302 \%$ Fuel Meat Plate 6

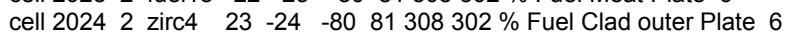


cell 20252 h2o $\quad 24-25 \quad-80 \quad 81308302 \%$ Water between Plates 6 and 7 cell 20262 zirc4 $25 \quad-26 \quad-80 \quad 81308302 \%$ Fuel Clad inner Plate 7 cell 20272 fuel19 $26 \quad-27 \quad-80 \quad 81308302 \%$ Fuel Meat Plate 7 $\begin{array}{llllllllll}\text { cell } 2028 & 2 & \text { zirc4 } & 27 & -28 & -80 & 81 & 308 & 302 & \%\end{array}$ Fuel Clad outer Plate 7 cell 20292 h2o $\quad 28 \quad-29 \quad-80 \quad 81308302 \%$ Water between Plates 7 and 8 cell 20302 zirc4 $29 \quad-30 \quad-80 \quad 81308302 \%$ Fuel Clad inner Plate 8 cell 20312 fuel20 $30 \quad-31 \quad-80 \quad 81308302 \%$ Fuel Meat Plate 8 $\begin{array}{lllllllll}\text { cell } 2032 & 2 & \text { zirc4 } & 31 & -32 & -80 & 81 & 308 & 302\end{array} \%$ Fuel Clad outer Plate 8 cell 20332 h2o $\quad 32-33 \quad-80 \quad 81308302 \%$ Water between Plates 8 and 9 cell 20342 zirc4 $33 \quad-34 \quad-80 \quad 81308302 \%$ Fuel Clad inner Plate 9 cell 20352 fuel21 $34 \quad-35 \quad-80 \quad 81308302 \%$ Fuel Meat Plate 9 cell 20362 zirc4 $35-36 \quad-80 \quad 81308302 \%$ Fuel Clad outer Plate 9 cell 20372 h2o $\quad 36-37 \quad-80 \quad 81308302 \%$ Water between Plates 9 and 10 cell 20382 zirc4 $37 \quad-38 \quad-80 \quad 81308302 \%$ Fuel Clad inner Plate 10 cell 20392 fuel22 $38 \quad-39 \quad-80 \quad 81308302 \%$ Fuel Meat Plate 10 cell 20402 zirc4 $39-40 \quad-80 \quad 81308302 \%$ Fuel Clad outer Plate 10 cell 20412 h2o $\quad 40 \quad-41 \quad-80 \quad 81308302 \%$ Water between Plates 10 and 11 cell 20422 zirc4 $41-42 \quad-80 \quad 81308302 \%$ Fuel Clad inner Plate 11 cell 20432 fuel23 $42 \quad-43 \quad-80 \quad 81308302 \%$ Fuel Meat Plate 11 cell 20442 zirc4 $43 \quad-44 \quad-80 \quad 81308302 \%$ Fuel Clad outer Plate 11 cell 20452 h2o $\quad 44-45 \quad-8081308302 \%$ Water between Plates 11 and 12 cell 20462 zirc4 $45 \quad-46 \quad-80 \quad 81308302 \%$ Fuel Clad inner Plate 12 cell 20472 fuel24 $46 \quad-47 \quad-8081308302 \%$ Fuel Meat Plate 12 cell 20482 zirc4 $47 \quad-48 \quad-80 \quad 81308302 \%$ Fuel Clad outer Plate 12

$\%$ *** ENE Octant ***

cell $2102 \quad 2$ zirc4 $1 \quad-2 \quad-80 \quad 81-307305 \%$ Fuel Clad inner Plate 1

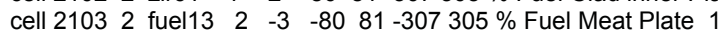
cell 21042 zirc4 $3 \quad-4 \quad-80 \quad 81-307 \quad 305 \%$ Fuel Clad outer Plate 1 cell 21052 h2o $\quad 4 \quad 4 \quad-5 \quad-80 \quad 81-307305 \%$ Water between Plates 1 and 2 cell 21062 zirc4 $5 \quad-6 \quad-80 \quad 81-307305 \%$ Fuel Clad inner Plate 2

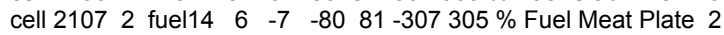
cell 21082 zirc4 $\quad 7 \quad-8 \quad-80 \quad 81-307 \quad 305 \%$ Fuel Clad outer Plate 2 cell 21092 h2o $\quad 8 \quad-9 \quad-80 \quad 81-307305 \%$ Water between Plates 2 and 3

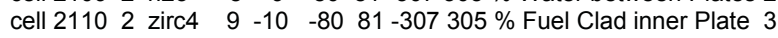
cell 21112 fuel15 $10-11-80 \quad 81-307305 \%$ Fuel Meat Plate 3 cell $2112 \quad 2$ zirc4 $11 \quad-12 \quad-80 \quad 81-307305 \%$ Fuel Clad outer Plate 3

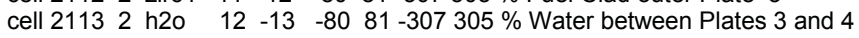
cell 21142 zirc4 $13 \quad-14 \quad-80 \quad 81-307305 \%$ Fuel Clad inner Plate 4 cell 21152 fuel16 $14 \quad-15 \quad-80 \quad 81-307305 \%$ Fuel Meat Plate 4

cell $2116 \quad 2$ zirc4 $15-16 \quad-80 \quad 81-307305 \%$ Fuel Clad outer Plate 4 cell 21172 h2o $16 \quad-17 \quad-80 \quad 81-307305 \%$ Water between Plates 4 and 5 cell 21182 zirc4 $17 \quad-18 \quad-80 \quad 81-307305 \%$ Fuel Clad inner Plate 5 cell 21192 fuel17 $18-19-80 \quad 81-307305 \%$ Fuel Meat Plate 5 cell 21202 zirc4 $19 \quad-20 \quad-80 \quad 81-307305 \%$ Fuel Clad outer Plate 5 $\begin{array}{llllllllll}\text { cell } 2121 & 2 & \text { h2o } & 20 & -21 & -80 & 81 & -307 & 305 & \%\end{array}$ Water between Plates 5 and 6 cell $2122 \quad 2$ zirc4 $21 \quad-22 \quad-80 \quad 81-307305 \%$ Fuel Clad inner Plate 6 cell 21232 fuel18 $22 \quad-23 \quad-80 \quad 81-307305 \%$ Fuel Meat Plate 6

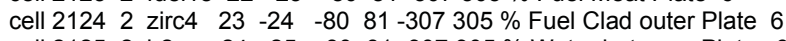

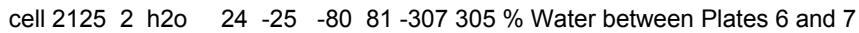
cell $2126 \quad 2$ zirc4 $25 \quad-26 \quad-80 \quad 81-307305 \%$ Fuel Clad inner Plate 7 cell 21272 fuel19 $26 \quad-27 \quad-80 \quad 81-307305 \%$ Fuel Meat Plate 7

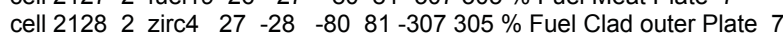

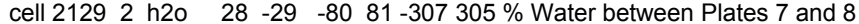
cell 21302 zirc4 $29 \quad-30 \quad-80 \quad 81-307305 \%$ Fuel Clad inner Plate 8

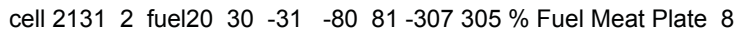
cell $2132 \quad 2$ zirc4 $31 \quad-32 \quad-80 \quad 81-307305 \%$ Fuel Clad outer Plate 8

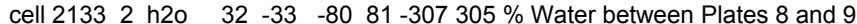
cell 21342 zirc4 $33 \quad-34 \quad-80 \quad 81-307305 \%$ Fuel Clad inner Plate 9 cell 21352 fuel21 $34 \quad-35 \quad-80 \quad 81-307305 \%$ Fuel Meat Plate 9 cell 21362 zirc4 $35-36 \quad-80 \quad 81-307305 \%$ Fuel Clad outer Plate 9

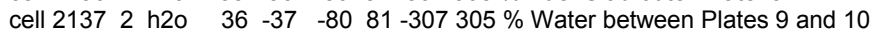
cell 21382 zirc4 $37 \quad-38 \quad-80 \quad 81-307305 \%$ Fuel Clad inner Plate 10 cell 21392 fuel22 $38 \quad-39 \quad-80 \quad 81-307305 \%$ Fuel Meat Plate 10 cell 21402 zirc4 $39 \quad-40 \quad-80 \quad 81-307305 \%$ Fuel Clad outer Plate 10 cell 21412 h2o $40 \quad-41 \quad-80 \quad 81-307305 \%$ Water between Plates 10 and 11 cell 21422 zirc4 $41 \quad-42 \quad-80 \quad 81-307305 \%$ Fuel Clad inner Plate 11 cell 21432 fuel23 $42 \quad-43 \quad-80 \quad 81-307305 \%$ Fuel Meat Plate 11 cell 21442 zirc4 $43 \quad-44 \quad-80 \quad 81-307305 \%$ Fuel Clad outer Plate 11 cell 21452 h2o $44 \quad-45 \quad-80 \quad 81-307305 \%$ Water between Plates 11 and 12 cell 21462 zirc4 $45 \quad-46 \quad-80 \quad 81-307305 \%$ Fuel Clad inner Plate 12 cell 21472 fuel24 $46 \quad-47 \quad-80 \quad 81-307305 \%$ Fuel Meat Plate 12 cell 21482 zirc4 $47 \quad-48 \quad-80 \quad 81-307305 \%$ Fuel Clad outer Plate 12

$\%{ }^{* * *}$ ESE Octant ***

cell $2202 \quad 2$ zirc4 $\quad 1 \quad-2 \quad-80 \quad 81-304311 \%$ Fuel Clad inner Plate 1 cell 22032 fuel13 $2 \quad-3 \quad-80 \quad 81-304311 \%$ Fuel Meat Plate 1 cell 22042 zirc4 $3 \quad-4 \quad-80 \quad 81-304311 \%$ Fuel Clad outer Plate 1 cell 22052 h2o $\quad 4 \quad-5 \quad-80 \quad 81-304311 \%$ Water between Plates 1 and 2 cell 22062 zirc4 $\quad 5 \quad-6 \quad-80 \quad 81-304311 \%$ Fuel Clad inner Plate 2 


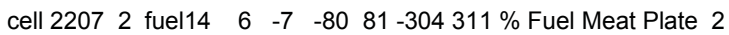
cell 22082 zirc4 $\quad 7 \quad-8 \quad-80 \quad 81-304311 \%$ Fuel Clad outer Plate 2

cell 22092 h2o $\quad 8 \quad-9 \quad-80 \quad 81-304311 \%$ Water between Plates 2 and 3

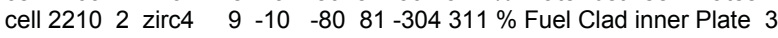

cell 22112 fuel15 $10-11 \quad-80 \quad 81-304311 \%$ Fuel Meat Plate 3

cell 22122 zirc4 $11-12 \quad-80 \quad 81-304311 \%$ Fuel Clad outer Plate 3

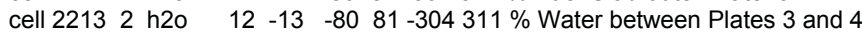

cell 22142 zirc4 $13-14 \quad-80 \quad 81-304311 \%$ Fuel Clad inner Plate 4

cell 22152 fuel16 $14 \quad-15 \quad-80 \quad 81-304311 \%$ Fuel Meat Plate 4

cell $2216 \quad 2$ zirc4 $15-16 \quad-80 \quad 81-304311 \%$ Fuel Clad outer Plate 4

cell $2217 \quad 2$ h2o $\quad 16 \quad-17 \quad-80 \quad 81-304311 \%$ Water between Plates 4 and 5

cell 22182 zirc4 $17-18 \quad-80 \quad 81-304311 \%$ Fuel Clad inner Plate 5

cell 22192 fuel17 $18-19 \quad-80 \quad 81-304311 \%$ Fuel Meat Plate 5

cell 22202 zirc4 $19-20 \quad-80 \quad 81-304311 \%$ Fuel Clad outer Plate 5

$\begin{array}{lllllllll}\text { cell } 2221 & 2 & \text { h2o } & 20 & -21 & -80 & 81 & -304 & 311 \% \text { Water between Plates } 5 \text { and } 6\end{array}$

cell 22222 zirc4 $21 \quad-22 \quad-80 \quad 81-304311 \%$ Fuel Clad inner Plate 6

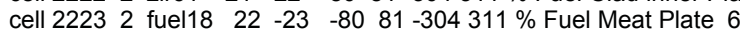

cell 22242 zirc4 $23-24 \quad-80 \quad 81-304311 \%$ Fuel Clad outer Plate 6

cell 22252 h2o $\quad 24-25 \quad-80 \quad 81-304311 \%$ Water between Plates 6 and 7

cell 22262 zirc4 $25 \quad-26 \quad-80 \quad 81-304311 \%$ Fuel Clad inner Plate 7

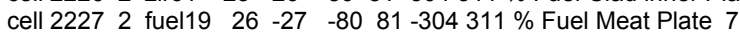

cell 22282 zirc4 $27 \quad-28 \quad-80 \quad 81-304311 \%$ Fuel Clad outer Plate 7

cell 22292 h2o $\quad 28-29 \quad-80 \quad 81-304311 \%$ Water between Plates 7 and 8

cell 22302 zirc4 $29-30 \quad-80 \quad 81-304311 \%$ Fuel Clad inner Plate 8

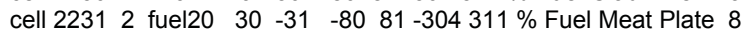

cell $2232 \quad 2$ zirc4 $31 \quad-32 \quad-80 \quad 81-304311 \%$ Fuel Clad outer Plate 8

cell 22332 h2o $\quad 32-33 \quad-80 \quad 81-304311 \%$ Water between Plates 8 and 9

cell 22342 zirc4 $33-34 \quad-80 \quad 81-304311 \%$ Fuel Clad inner Plate 9

cell 22352 fuel21 $34 \quad-35 \quad-80 \quad 81-304311 \%$ Fuel Meat Plate 9

cell 22362 zirc4 $35 \quad-36 \quad-80 \quad 81-304311 \%$ Fuel Clad outer Plate 9

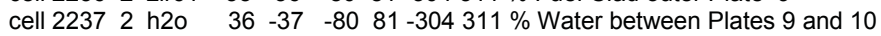

cell 22382 zirc4 $37 \quad-38 \quad-80 \quad 81-304311 \%$ Fuel Clad inner Plate 10

cell 22392 fuel22 38 -39 $-80 \quad 81-304311 \%$ Fuel Meat Plate 10

cell 22402 zirc4 $39-40 \quad-80 \quad 81-304311 \%$ Fuel Clad outer Plate 10

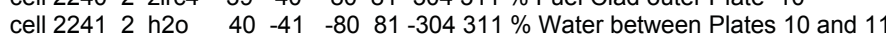

cell $2242 \quad 2$ zirc4 $41-42 \quad-80 \quad 81-304311 \%$ Fuel Clad inner Plate 11

cell 22432 fuel23 $42 \quad-43 \quad-80 \quad 81-304311 \%$ Fuel Meat Plate 11

cell 22442 zirc4 $43 \quad-44 \quad-80 \quad 81-304311 \%$ Fuel Clad outer Plate 11

cell 22452 h2o $\quad 44 \quad-45 \quad-80 \quad 81-304311 \%$ Water between Plates 11 and 12

cell 22462 zirc4 $45 \quad-46 \quad-80 \quad 81-304311 \%$ Fuel Clad inner Plate 12

cell 22472 fuel24 $46 \quad-47 \quad-80 \quad 81-304311 \%$ Fuel Meat Plate 12

cell 22482 zirc4 $47 \quad-48 \quad-80 \quad 81-304311 \%$ Fuel Clad outer Plate 12

$\%$ *** SSE Octant ***

cell $2302 \quad 2$ zirc4 $1 \quad-2 \quad-80 \quad 81-310302 \%$ Fuel Clad inner Plate 1

cell 23032 fuel13 $2 \quad-3 \quad-80 \quad 81-310302 \%$ Fuel Meat Plate 1

cell 23042 zirc4 $3 \quad 3 \quad-4 \quad-80 \quad 81-310302 \%$ Fuel Clad outer Plate 1

cell 23052 h2o $\quad 4 \quad-5 \quad-80 \quad 81-310302 \%$ Water between Plates 1 and 2

cell 23062 zirc4 $5 \quad 5 \quad-6 \quad-80 \quad 81-310302 \%$ Fuel Clad inner Plate 2

cell 23072 fuel14 $6 \begin{array}{lllllll}6 & -7 & -80 & 81 & -310 & 302 \% & \text { Fuel Meat Plate } 2\end{array}$

cell $2308 \quad 2$ zirc4 $\quad 7 \quad-8 \quad-80 \quad 81-310302 \%$ Fuel Clad outer Plate 2

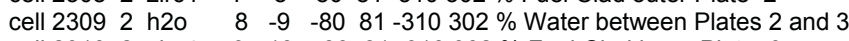

cell 23102 zirc4 $9-10 \quad-80 \quad 81-310302 \%$ Fuel Clad inner Plate 3

cell 23112 fuel15 $10-11 \quad-80 \quad 81-310302 \%$ Fuel Meat Plate 3

cell 23122 zirc4 $11-12 \quad-80 \quad 81-310302 \%$ Fuel Clad outer Plate 3

cell 23132 h2o $12 \quad-13 \quad-80 \quad 81-310302 \%$ Water between Plates 3 and 4

cell 23142 zirc4 $13-14 \quad-80 \quad 81-310302 \%$ Fuel Clad inner Plate 4

cell 23152 fuel16 $14 \quad-15 \quad-80 \quad 81-310302 \%$ Fuel Meat Plate 4

cell 23162 zirc4 $15-16 \quad-80 \quad 81-310302 \%$ Fuel Clad outer Plate 4

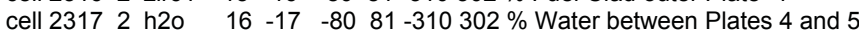

cell 23182 zirc4 $17-18 \quad-80 \quad 81-310302 \%$ Fuel Clad inner Plate 5

cell 23192 fuel17 $18 \quad-19 \quad-80 \quad 81-310302 \%$ Fuel Meat Plate 5

cell 23202 zirc4 $19-20 \quad-80 \quad 81-310302 \%$ Fuel Clad outer Plate 5

cell $2321 \quad 2$ h2o $\quad 20-21 \quad-80 \quad 81-310302 \%$ Water between Plates 5 and 6

cell 23222 zirc4 $21 \quad-22 \quad-80 \quad 81-310302 \%$ Fuel Clad inner Plate 6

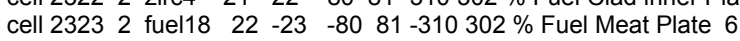

cell 23242 zirc4 $23 \quad-24 \quad-80 \quad 81-310302 \%$ Fuel Clad outer Plate 6

cell $2325 \quad 2$ h2o $\quad 24 \quad-25 \quad-80 \quad 81-310302 \%$ Water between Plates 6 and 7

cell 23262 zirc4 $25 \quad-26 \quad-80 \quad 81-310302 \%$ Fuel Clad inner Plate 7

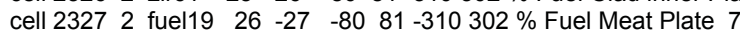

cell 23282 zirc4 $27 \quad-28 \quad-80 \quad 81-310302 \%$ Fuel Clad outer Plate 7

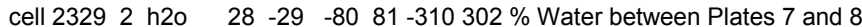

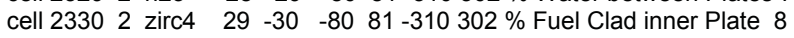

cell 23312 fuel20 $30 \quad-31 \quad-80 \quad 81-310302 \%$ Fuel Meat Plate 8

cell 23322 zirc4 $31 \quad-32 \quad-80 \quad 81-310302 \%$ Fuel Clad outer Plate 8

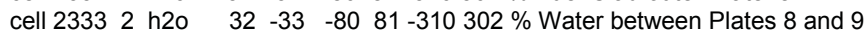

cell 23342 zirc4 $33 \quad-34 \quad-80 \quad 81-310302 \%$ Fuel Clad inner Plate 9

cell 23352 fuel21 $34 \quad-35 \quad-80 \quad 81-310302 \%$ Fuel Meat Plate 9

cell $2336 \quad 2$ zirc4 $35 \quad-36 \quad-80 \quad 81-310302 \%$ Fuel Clad outer Plate 9

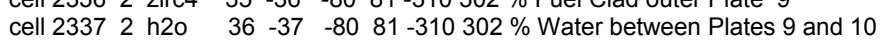


cell 23382 zirc4 $37 \quad-38 \quad-80 \quad 81-310302 \%$ Fuel Clad inner Plate 10 cell 23392 fuel22 $38 \quad-39 \quad-80 \quad 81-310302 \%$ Fuel Meat Plate 10

cell 23402 zirc4 $39-40 \quad-80 \quad 81-310302 \%$ Fuel Clad outer Plate 10

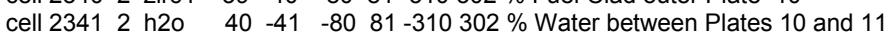

cell $2342 \quad 2$ zirc4 $41 \quad-42 \quad-80 \quad 81-310302 \%$ Fuel Clad inner Plate 11

cell 23432 fuel23 $42 \quad-43 \quad-80 \quad 81-310302 \%$ Fuel Meat Plate 11

cell 23442 zirc4 $43 \quad-44 \quad-80 \quad 81-310302 \%$ Fuel Clad outer Plate 11

cell $2345 \quad 2$ h2o $\quad 44-45 \quad-80 \quad 81-310302 \%$ Water between Plates 11 and 12

cell 23462 zirc4 $45 \quad-46 \quad-80 \quad 81-310302 \%$ Fuel Clad inner Plate 12

cell 23472 fuel24 $46 \quad-47 \quad-80 \quad 81-310302 \%$ Fuel Meat Plate 12

cell 23482 zirc4 $47 \quad-48 \quad-80 \quad 81-310302 \%$ Fuel Clad outer Plate 12

$\%$ *** SSW Octant ***

cell 24022 zirc4 $1-2 \quad-80 \quad 81-301-307 \%$ Fuel Clad inner Plate 1

cell 24032 fuel13 $2 \quad-3 \quad-80 \quad 81-301-307 \%$ Fuel Meat Plate 1

cell 24042 zirc4 $3 \quad-4 \quad-80 \quad 81-301-307 \%$ Fuel Clad outer Plate 1

cell 24052 h2o $\quad 4 \quad-5-80 \quad 81-301-307 \%$ Water between Plates 1 and 2

cell 24062 zirc4 5 - 6 -80 $81-301-307 \%$ Fuel Clad inner Plate 2

cell 24072 fuel14 6 -7 $-80 \quad 81-301-307 \%$ Fuel Meat Plate 2

cell $2408 \quad 2$ zirc4 $\quad 7 \quad-8 \quad-80 \quad 81-301-307 \%$ Fuel Clad outer Plate 2

cell 24092 h2o $\quad 8 \quad-9-80 \quad 81-301-307 \%$ Water between Plates 2 and 3

cell 24102 zirc4 $9-10-80 \quad 81-301-307 \%$ Fuel Clad inner Plate 3

cell 24112 fuel15 $10-11-8081-301-307 \%$ Fuel Meat Plate 3

cell 24122 zirc4 $11-12-80 \quad 81-301-307 \%$ Fuel Clad outer Plate 3

cell 24132 h2o $12-13-80 \quad 81-301-307 \%$ Water between Plates 3 and 4

cell 24142 zirc4 $13-14-80 \quad 81-301-307 \%$ Fuel Clad inner Plate 4

cell 24152 fuel16 $14-15-80 \quad 81-301-307 \%$ Fuel Meat Plate 4

cell 24162 zirc4 $15-16-80 \quad 81-301-307 \%$ Fuel Clad outer Plate 4

cell 24172 h2o $\quad 16-17-80 \quad 81-301-307 \%$ Water between Plates 4 and 5

cell 24182 zirc4 $17-18-80 \quad 81-301-307 \%$ Fuel Clad inner Plate 5

cell 24192 fuel17 $18-19-80 \quad 81-301-307 \%$ Fuel Meat Plate 5

cell 24202 zirc4 $19-20-80 \quad 81-301-307 \%$ Fuel Clad outer Plate 5

cell 24212 h2o $\quad 20-21-80 \quad 81-301-307 \%$ Water between Plates 5 and 6

cell 24222 zirc4 $21 \quad-22 \quad-80 \quad 81-301-307 \%$ Fuel Clad inner Plate 6

cell 24232 fuel18 $22 \quad-23 \quad-80 \quad 81-301-307 \%$ Fuel Meat Plate 6

cell 24242 zirc4 $23-24-80 \quad 81-301-307 \%$ Fuel Clad outer Plate 6

cell 24252 h2o $24-25-80 \quad 81-301-307 \%$ Water between Plates 6 and 7

cell 24262 zirc4 $25 \quad-26-80 \quad 81-301-307 \%$ Fuel Clad inner Plate 7

cell 24272 fuel19 $26 \quad-27 \quad-80 \quad 81-301-307 \%$ Fuel Meat Plate 7

cell 24282 zirc4 $27-28-80 \quad 81-301-307 \%$ Fuel Clad outer Plate 7

$\begin{array}{llllllllll}\text { cell } 2429 & 2 & \text { h2o } & 28 & -29 & -80 & 81 & -301 & -307 & \%\end{array}$ Water between Plates 7 and 8

cell 24302 zirc4 $29-30-80 \quad 81-301-307 \%$ Fuel Clad inner Plate 8

cell 24312 fuel20 $30 \quad-31 \quad-80 \quad 81-301-307 \%$ Fuel Meat Plate 8

cell 24322 zirc4 $31 \quad-32 \quad-80 \quad 81-301-307 \%$ Fuel Clad outer Plate 8

$\begin{array}{lllllllll}\text { cell } 2433 & 2 & \text { h2o } & 32 & -33 & -80 & 81 & -301 & -307 \% \text { Water between Plates } 8 \text { and } 9\end{array}$

cell 24342 zirc4 $33-34-80 \quad 81-301-307 \%$ Fuel Clad inner Plate 9

cell 24352 fuel21 $34-35-80 \quad 81-301-307 \%$ Fuel Meat Plate 9

cell 24362 zirc4 $35 \quad-36-80 \quad 81-301-307 \%$ Fuel Clad outer Plate 9

$\begin{array}{lllllllllll}\text { cell } 2437 & 2 & \text { h2o } & 36 & -37 & -80 & 81 & -301 & -307 \% \text { Water between Plates } 9 \text { and } 10\end{array}$

cell 24382 zirc4 $37-38-80 \quad 81-301-307 \%$ Fuel Clad inner Plate 10

cell 24392 fuel22 38 -39 $-80 \quad 81-301-307 \%$ Fuel Meat Plate 10

cell 24402 zirc4 $39-40-80 \quad 81-301-307 \%$ Fuel Clad outer Plate 10

cell 24412 h2o $40-41-80 \quad 81-301-307 \%$ Water between Plates 10 and 11

cell 24422 zirc4 $41-42-80 \quad 81-301-307 \%$ Fuel Clad inner Plate 11

cell 24432 fuel23 $42 \quad-43 \quad-80 \quad 81-301-307 \%$ Fuel Meat Plate 11

cell 24442 zirc4 $43-44-80 \quad 81-301-307 \%$ Fuel Clad outer Plate 11

cell 24452 h2o $44-45-80 \quad 81-301-307 \%$ Water between Plates 11 and 12

cell 24462 zirc4 $45-46-80 \quad 81-301-307 \%$ Fuel Clad inner Plate 12

cell 24472 fuel24 $46-47-80 \quad 81-301-307 \%$ Fuel Meat Plate 12

cell $2448 \quad 2$ zirc4 $47 \quad-48 \quad-80 \quad 81-301-307 \%$ Fuel Clad outer Plate 12

$\%$ *** WSW Octant ***

cell 25022 zirc4 $1 \quad-2 \quad-80 \quad 81-304308 \%$ Fuel Clad inner Plate 1

cell 25032 fuel13 $2 \quad-3 \quad-80 \quad 81-304308 \%$ Fuel Meat Plate 1

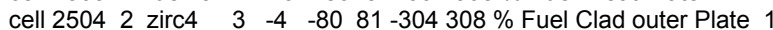

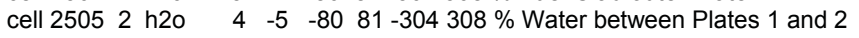

cell 25062 zirc4 $\quad 5 \quad-6 \quad-80 \quad 81-304308 \%$ Fuel Clad inner Plate 2

cell 25072 fuel14 $6 \begin{array}{llllll}-7 & -80 & 81 & -304 & 308 \% & \text { Fuel Meat Plate } 2\end{array}$

cell $2508 \quad 2$ zirc4 $\quad 7 \quad-8 \quad-80 \quad 81-304308 \%$ Fuel Clad outer Plate 2

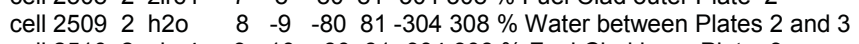

cell 25102 zirc4 $9-10 \quad-80 \quad 81-304308 \%$ Fuel Clad inner Plate 3

cell 25112 fuel15 $10-11 \quad-80 \quad 81-304308 \%$ Fuel Meat Plate 3

cell 25122 zirc4 $11 \quad-12 \quad-80 \quad 81-304308 \%$ Fuel Clad outer Plate 3

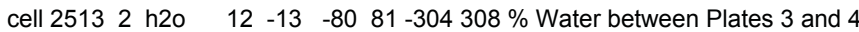

cell 25142 zirc4 $13-14 \quad-80 \quad 81-304308 \%$ Fuel Clad inner Plate 4

cell 25152 fuel16 $14-15 \quad-80 \quad 81-304308 \%$ Fuel Meat Plate 4

cell 25162 zirc4 $15-16 \quad-80 \quad 81-304308 \%$ Fuel Clad outer Plate 4

cell $2517 \quad 2$ h2o $\quad 16 \quad-17 \quad-80 \quad 81-304308 \%$ Water between Plates 4 and 5

cell 25182 zirc4 $17-18 \quad-80 \quad 81-304308 \%$ Fuel Clad inner Plate 5

cell 25192 fuel17 $18 \quad-19 \quad-80 \quad 81-304308 \%$ Fuel Meat Plate 5 
cell 25202 zirc4 $19-20 \quad-80 \quad 81-304308 \%$ Fuel Clad outer Plate 5 cell 25212 h2o $\quad 20 \quad-21 \quad-80 \quad 81-304308 \%$ Water between Plates 5 and 6 cell 25222 zirc4 $21 \quad-22 \quad-80 \quad 81-304308 \%$ Fuel Clad inner Plate 6 cell 25232 fuel18 $22 \quad-23 \quad-80 \quad 81-304308 \%$ Fuel Meat Plate 6 cell 25242 zirc4 $23 \quad-24 \quad-80 \quad 81-304308 \%$ Fuel Clad outer Plate 6 cell 25252 h2o $\quad 24 \quad-25 \quad-80 \quad 81-304308 \%$ Water between Plates 6 and 7 cell 25262 zirc4 $25 \quad-26 \quad-80 \quad 81-304308 \%$ Fuel Clad inner Plate 7 cell 25272 fuel19 $26 \quad-27 \quad-80 \quad 81-304308 \%$ Fuel Meat Plate 7 cell 25282 zirc4 $27 \quad-28 \quad-80 \quad 81-304308 \%$ Fuel Clad outer Plate 7

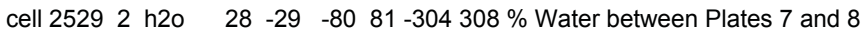
cell 25302 zirc4 $29 \quad-30 \quad-80 \quad 81-304308 \%$ Fuel Clad inner Plate 8 cell 25312 fuel20 $30 \quad-31 \quad-80 \quad 81-304308 \%$ Fuel Meat Plate 8 cell 25322 zirc4 $31 \quad-32 \quad-80 \quad 81-304308 \%$ Fuel Clad outer Plate 8

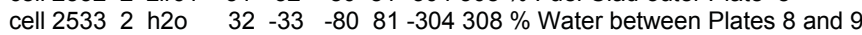
cell 25342 zirc4 $33 \quad-34 \quad-80 \quad 81-304308 \%$ Fuel Clad inner Plate 9 cell 25352 fuel21 $34 \quad-35 \quad-80 \quad 81-304308 \%$ Fuel Meat Plate 9 cell 25362 zirc4 $35-36 \quad-80 \quad 81-304308 \%$ Fuel Clad outer Plate 9

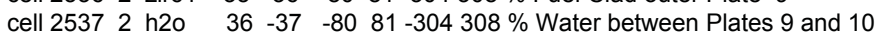
cell $2538 \quad 2$ zirc4 $37 \quad-38 \quad-80 \quad 81-304308 \%$ Fuel Clad inner Plate 10 cell 25392 fuel22 $38 \quad-39 \quad-80 \quad 81-304308 \%$ Fuel Meat Plate 10 cell 25402 zirc4 $39-40 \quad-80 \quad 81-304308 \%$ Fuel Clad outer Plate 10 cell 25412 h2o $\quad 40-41 \quad-80 \quad 81-304308 \%$ Water between Plates 10 and 11 cell 25422 zirc4 $41 \quad-42 \quad-80 \quad 81-304308 \%$ Fuel Clad inner Plate 11 cell 25432 fuel23 $42 \quad-43 \quad-80 \quad 81-304308 \%$ Fuel Meat Plate 11 cell 25442 zirc4 $43 \quad-44 \quad-80 \quad 81-304308 \%$ Fuel Clad outer Plate 11 cell 25452 h2o $\quad 44 \quad-45 \quad-80 \quad 81-304308 \%$ Water between Plates 11 and 12 cell 25462 zirc4 $45-46 \quad-80 \quad 81-304308 \%$ Fuel Clad inner Plate 12 cell 25472 fuel24 $46 \quad-47 \quad-80 \quad 81-304308 \%$ Fuel Meat Plate 12 cell $2548 \quad 2$ zirc4 $47 \quad-48 \quad-80 \quad 81-304 \quad 308 \%$ Fuel Clad outer Plate 12

$\%$ *** WNW Octant ***

cell $2602 \quad 2$ zirc4 $1-2 \quad-80 \quad 81-310305 \%$ Fuel Clad inner Plate 1 cell 26032 fuel13 $22 \quad-3 \quad-80 \quad 81-310305 \%$ Fuel Meat Plate 1 cell $2604 \quad 2$ zirc4 $\quad 3 \quad-4 \quad-80 \quad 81-310305 \%$ Fuel Clad outer Plate 1

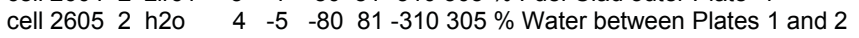
cell 26062 zirc4 $5 \quad-6 \quad-80 \quad 81-310305 \%$ Fuel Clad inner Plate 2 cell $2607 \quad 2$ fuel14 $6 \begin{array}{llllll}-7 & -80 & 81 & -310 & 305 \% & \text { Fuel Meat Plate } 2\end{array}$ cell 26082 zirc4 $\quad 7 \quad-8 \quad-80 \quad 81-310305 \%$ Fuel Clad outer Plate 2 cell 26092 h2o $\quad 8 \quad-9 \quad-80 \quad 81-310305 \%$ Water between Plates 2 and 3 cell 26102 zirc4 $9-10 \quad-80 \quad 81-310305 \%$ Fuel Clad inner Plate 3 cell 26112 fuel15 $10-11 \quad-80 \quad 81-310305 \%$ Fuel Meat Plate 3 cell 26122 zirc4 $11-12 \quad-80 \quad 81-310305 \%$ Fuel Clad outer Plate 3 cell 26132 h2o $\quad 12-13 \quad-80 \quad 81-310305 \%$ Water between Plates 3 and 4 cell 26142 zirc4 $13-14 \quad-80 \quad 81-310305 \%$ Fuel Clad inner Plate 4 cell 26152 fuel16 $14-15 \quad-80 \quad 81-310305 \%$ Fuel Meat Plate 4 cell 26162 zirc4 $15 \quad-16 \quad-80 \quad 81-310305 \%$ Fuel Clad outer Plate 4 cell $2617 \quad 2$ h2o $\quad 16 \quad-17 \quad-80 \quad 81-310305 \%$ Water between Plates 4 and 5 cell 26182 zirc4 $17 \quad-18 \quad-80 \quad 81-310305 \%$ Fuel Clad inner Plate 5 cell 26192 fuel17 18 -19 $-80 \quad 81-310305 \%$ Fuel Meat Plate 5 cell 26202 zirc4 $19-20 \quad-80 \quad 81-310305 \%$ Fuel Clad outer Plate 5

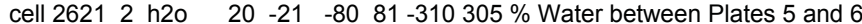

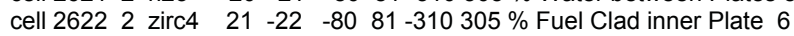
cell 26232 fuel18 $22 \quad-23 \quad-80 \quad 81-310305 \%$ Fuel Meat Plate 6 cell 26242 zirc4 $23 \quad-24 \quad-80 \quad 81-310305 \%$ Fuel Clad outer Plate 6 cell 26252 h2o $\quad 24 \quad-25 \quad-80 \quad 81-310305 \%$ Water between Plates 6 and 7 cell 26262 zirc4 $25 \quad-26 \quad-80 \quad 81-310305 \%$ Fuel Clad inner Plate 7 cell 26272 fuel19 $26 \quad-27 \quad-80 \quad 81-310305 \%$ Fuel Meat Plate 7 cell 26282 zirc4 $27 \quad-28 \quad-80 \quad 81-310305 \%$ Fuel Clad outer Plate 7

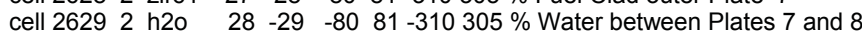
cell 26302 zirc4 $29 \quad-30 \quad-80 \quad 81-310305 \%$ Fuel Clad inner Plate 8 cell 26312 fuel20 $30 \quad-31 \quad-80 \quad 81-310305 \%$ Fuel Meat Plate 8 cell 26322 zirc4 $31 \quad-32 \quad-80 \quad 81-310305 \%$ Fuel Clad outer Plate 8

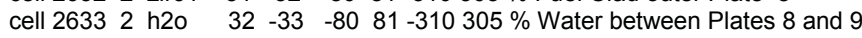
cell 26342 zirc4 $33 \quad-34 \quad-80 \quad 81-310305 \%$ Fuel Clad inner Plate 9 cell 26352 fuel21 $34 \quad-35 \quad-80 \quad 81-310305 \%$ Fuel Meat Plate 9 cell 26362 zirc4 $35 \quad-36 \quad-80 \quad 81-310305 \%$ Fuel Clad outer Plate 9

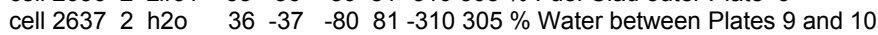
cell 26382 zirc4 $37 \quad-38 \quad-80 \quad 81-310305 \%$ Fuel Clad inner Plate 10 cell 26392 fuel22 $38 \quad-39 \quad-80 \quad 81-310305 \%$ Fuel Meat Plate 10 cell 26402 zirc4 $39 \quad-40 \quad-80 \quad 81-310305 \%$ Fuel Clad outer Plate 10 cell 26412 h2o $\quad 40 \quad-41 \quad-80 \quad 81-310305 \%$ Water between Plates 10 and 11 cell 26422 zirc4 $41 \quad-42 \quad-80 \quad 81-310305 \%$ Fuel Clad inner Plate 11 cell 26432 fuel23 $42 \quad-43 \quad-80 \quad 81-310305 \%$ Fuel Meat Plate 11 cell $2644 \quad 2$ zirc4 $43 \quad-44 \quad-80 \quad 81-310305 \%$ Fuel Clad outer Plate 11 cell 26452 h2o $\quad 44-45 \quad-80 \quad 81-310305 \%$ Water between Plates 11 and 12 cell $2646 \quad 2$ zirc4 $45 \quad-46 \quad-80 \quad 81-310305 \%$ Fuel Clad inner Plate 12 cell 26472 fuel24 $46 \quad-47 \quad-80 \quad 81-310305 \%$ Fuel Meat Plate 12 cell 26482 zirc4 $47 \quad-48 \quad-80 \quad 81-310305 \%$ Fuel Clad outer Plate 12

$\%{ }^{* * *}$ NNW Octant *** 
cell 27022 zirc4 $1 \quad-2 \quad-80 \quad 81311-301 \%$ Fuel Clad inner Plate 1 cell 27032 fuel13 $2 \quad-3 \quad-80 \quad 81311-301 \%$ Fuel Meat Plate 1

cell $2704 \quad 2$ zirc4 $3 \quad-4 \quad-80 \quad 81311-301 \%$ Fuel Clad outer Plate 1

cell 27052 h2o $4 \quad 4 \quad-5-80 \quad 81311-301 \%$ Water between Plates 1 and 2

cell 27062 zirc4 $5 \quad-6-8081311-301 \%$ Fuel Clad inner Plate 2

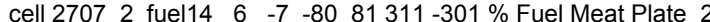

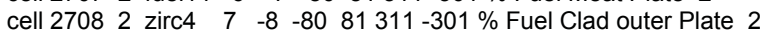

cell 27092 h2o $\quad 8 \quad-9 \quad-80 \quad 81311-301 \%$ Water between Plates 2 and 3

cell 27102 zirc4 $9-10-8081311-301 \%$ Fuel Clad inner Plate 3

cell 27112 fuel15 $10-11-80 \quad 81311-301 \%$ Fuel Meat Plate 3

cell 27122 zirc4 $11 \quad-12-80 \quad 81311-301 \%$ Fuel Clad outer Plate 3

cell 27132 h2o $12-13-8081311-301 \%$ Water between Plates 3 and 4

cell 27142 zirc4 $13-14-8081311-301 \%$ Fuel Clad inner Plate 4

cell 27152 fuel16 $14 \quad-15-80 \quad 81311-301 \%$ Fuel Meat Plate 4

cell $2716 \quad 2$ zirc4 $15 \quad-16 \quad-80 \quad 81311-301 \%$ Fuel Clad outer Plate 4

cell 27172 h2o $\quad 16-17-80 \quad 81311-301 \%$ Water between Plates 4 and 5

cell 27182 zirc4 $17-18 \quad-80 \quad 81311-301 \%$ Fuel Clad inner Plate 5

cell 27192 fuel17 $18-19-80 \quad 81311-301 \%$ Fuel Meat Plate 5

cell 27202 zirc4 $19-20-80 \quad 81311-301 \%$ Fuel Clad outer Plate 5

cell 27212 h2o $\quad 20-21-80 \quad 81311-301 \%$ Water between Plates 5 and 6

cell $2722 \quad 2$ zirc4 $21 \quad-22 \quad-80 \quad 81311-301 \%$ Fuel Clad inner Plate 6

cell 27232 fuel18 $22 \quad-23 \quad-80 \quad 81311-301 \%$ Fuel Meat Plate 6

cell 27242 zirc4 $23 \quad-24 \quad-80 \quad 81311-301 \%$ Fuel Clad outer Plate 6

cell $2725 \quad 2$ h2o $24 \quad-25-80 \quad 81311-301 \%$ Water between Plates 6 and 7

cell $2726 \quad 2$ zirc4 $25 \quad-26 \quad-80 \quad 81311-301 \%$ Fuel Clad inner Plate 7

cell 27272 fuel19 $26 \quad-27 \quad-80 \quad 81311-301 \%$ Fuel Meat Plate 7

cell $2728 \quad 2$ zirc4 $27 \quad-28 \quad-80 \quad 81311-301 \%$ Fuel Clad outer Plate 7

$\begin{array}{lllllllllll}\text { cell } 2729 & 2 & \text { h2o } & 28 & -29 & -80 & 81 & 311 & -301 \% \text { Water between Plates } 7 \text { and } 8\end{array}$

$\begin{array}{lllllllll}\text { cell } 2730 & 2 & \text { zirc4 } & 29 & -30 & -80 & 81 & 311 & -301 \% \text { Fuel Clad inner Plate } 8\end{array}$

cell 27312 fuel20 $30 \quad-31 \quad-80 \quad 81311-301 \%$ Fuel Meat Plate 8

cell 27322 zirc4 $31 \quad-32 \quad-80 \quad 81311-301 \%$ Fuel Clad outer Plate 8

$\begin{array}{llllllllll}\text { cell } 2733 & 2 & \text { h2o } & 32 & -33 & -80 & 81 & 311 & -301 \% \text { Water between Plates } 8 \text { and } 9\end{array}$

cell 27342 zirc4 $33-34-8081311-301 \%$ Fuel Clad inner Plate 9

cell 27352 fuel21 $34-35-80 \quad 81311-301 \%$ Fuel Meat Plate 9

cell 27362 zirc4 $35-36-80 \quad 81311-301 \%$ Fuel Clad outer Plate 9

cell $2737 \quad 2$ h2o $\quad 36 \quad-37 \quad-80 \quad 81311-301 \%$ Water between Plates 9 and 10

cell 27382 zirc4 $37 \quad-38$-80 $81311-301 \%$ Fuel Clad inner Plate 10

cell 27392 fuel22 $38 \quad-39-80 \quad 81311-301 \%$ Fuel Meat Plate 10

cell 27402 zirc4 $39-40-80 \quad 81311-301 \%$ Fuel Clad outer Plate 10

cell 27412 h2o $\quad 40 \quad-41-80 \quad 81311-301 \%$ Water between Plates 10 and 11

cell 27422 zirc4 $41 \quad-42-80 \quad 81311-301 \%$ Fuel Clad inner Plate 11

cell 27432 fuel23 $42 \quad-43-80 \quad 81311-301 \%$ Fuel Meat Plate 11

cell 27442 zirc4 $43 \quad-44-80 \quad 81311-301 \%$ Fuel Clad outer Plate 11

cell 27452 h2o $\quad 44 \quad-45-80 \quad 81311-301 \%$ Water between Plates 11 and 12

cell 27462 zirc4 $45-46-8081311-301 \%$ Fuel Clad inner Plate 12

cell 27472 fuel24 $46 \quad-47 \quad-80 \quad 81311-301 \%$ Fuel Meat Plate 12

cell 27482 zirc4 $47 \quad-48 \quad-80 \quad 81311-301 \%$ Fuel Clad outer Plate 12

$\%$ Other materials above and below fuel assembly

cell 5772 h2o $\quad 1-128 \quad 64-203 \quad \%$ water above fuel assembly

$\begin{array}{lllllll}\text { cell } 578 & 2 & \text { h2o } & 1 & -128 & -65 & 202\end{array} \%$ water below fuel assembly

$\%$ Upper and lower plates without fuel homogenized

cell 5852 TopPlat $1-128 \quad 80-62 \%$ upper end plates

cell 5862 BotPlat $1-128-8163 \quad \%$ lower end plates

$\%$ Upper and lower end boxes homogenized

cell 5872 TopBox $1-128 \quad 62-64 \quad \%$ upper end box

cell 5882 BotBox $1-128 \quad-63 \quad 65 \quad \%$ lower end box

$\%$ Side plates (fused adjacent plates)

cell 5902 zirc4 1 -48 $301-302303-80 \quad 81 \%$ N sideplates

cell 5912 zirc4 1 -48 307 -308 $303-80 \quad 81 \%$ NE sideplates

cell 5922 zirc4 1 -48 $304-305 \quad 300-80 \quad 81 \%$ E sideplates

cell 5932 zirc4 $1-48310-311-303-80 \quad 81 \%$ SE sideplates

cell 5942 zirc4 1 -48 $301-302-303-80 \quad 81 \%$ S sideplates

cell 5952 zirc4 $1-48307-308-303-80 \quad 81 \%$ SW sideplates

cell 5962 zirc4 1 -48 $304-305-300-80 \quad 81 \%$ W sideplates

cell 5972 zirc4 $1-48 \quad 310-311 \quad 303-80 \quad 81 \%$ NW sideplates

cell 2004 AIRack -201 \% large area of Rack. used for filling lat.

lat 1504002

1 0. 0.1

634. 0. 222222

cell 1810 fill $150-205 \quad 202-203 \quad \%$ inside pressure vessel cell 1820 al6061 $205-206 \quad 202-203 \%$ pressure vessel cell 1830 d2o $206-201 \quad 202-203 \quad \%$ d2o outside pressure vessel $\%$ 
\% ------ Void Outside Core ------

cell 90010 outside $201203 \%$ rest of universe

cell 90020 outside $201-202 \%$ rest of universe

cell 90030 outside $-201203 \%$ rest of universe

cell 90040 outside $-201-202 \%$ rest of universe

cell 90050 outside $201-203202 \%$ rest of universe

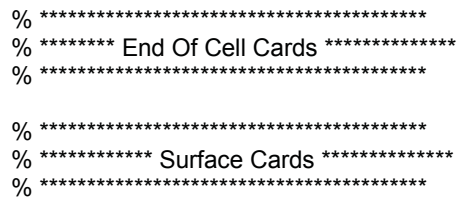

$\%{ }^{* * *}$ Fuel Plate Cylindrical surfaces Large Central Location **** surf 1 cyl $0.0 \quad 0.0 \quad 9.6000 \quad \%$ Fuel Cld inner Plate 1 $\begin{array}{lllll}\text { surf } 2 \text { cyl } & 0.0 & 0.0 & 9.6375 & \% \text { Fuel Mt inner Plate } 1\end{array}$ surf 3 cyl $0.0 \quad 0.0 \quad 9.6875 \quad \%$ Fuel Mt outer Plate 1 surf 4 cyl $0.0 \quad 0.0 \quad 9.7250 \quad \%$ Fuel Cld outer Plate 1 surf 5 cyl $0.0 \quad 0.0 \quad 9.9250 \quad \%$ Fuel Cld inner Plate 2 surf 6 cyl $0.0 \quad 0.0 \quad 9.9625 \quad$ \% Fuel Mt inner Plate 2

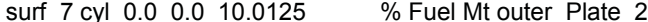
surf 8 cyl $0.0 \quad 0.010 .0500 \quad \%$ Fuel Cld outer Plate 2 surf 9 cyl $0.0 \quad 0.0 \quad 10.2500 \quad \%$ Fuel Cld inner Plate 3 surf 10 cyl $0.0 \quad 0.0 \quad 10.2875 \quad \%$ Fuel Mt inner Plate 3 surf $11 \mathrm{cyl} \quad 0.0 \quad 0.0 \quad 10.3375 \quad \%$ Fuel Mt outer Plate 3 surf 12 cyl $0.0 \quad 0.0 \quad 10.3750 \quad \%$ Fuel Cld outer Plate 3 surf 13 cyl $0.0 \quad 0.010 .5750 \quad \%$ Fuel Cld inner Plate 4 surf 14 cyl $0.0 \quad 0.0 \quad 10.6125 \quad \%$ Fuel Mt inner Plate 4 surf 15 cyl $0.0 \quad 0.010 .6625 \quad \%$ Fuel Mt outer Plate 4 surf 16 cyl $0.0 \quad 0.0 \quad 10.7000 \quad \%$ Fuel Cld outer Plate 4 surf 17 cyl $0.0 \quad 0.0 \quad 10.9000 \quad \%$ Fuel Cld inner Plate 5 surf 18 cyl $0.0 \quad 0.0 \quad 10.9375 \quad \%$ Fuel Mt inner Plate 5 surf 19 cyl $0.0 \quad 0.010 .9875 \quad \%$ Fuel Mt outer Plate 5 surf 20 cyl $0.0 \quad 0.011 .0250 \quad \%$ Fuel Cld outer Plate 5 surf $21 \mathrm{cyl} \quad 0.0 \quad 0.0 \quad 11.2250 \quad \%$ Fuel Cld inner Plate 6 surf 22 cyl $\begin{array}{llll}0.0 & 0.0 & 11.2625 & \% \text { Fuel Mt inner Plate } 6\end{array}$ surf 23 cyl $0.0 \quad 0.0 \quad 11.3125 \quad \%$ Fuel Mt outer Plate 6 surf 24 cyl $\begin{array}{llll}0.0 & 0.0 & 11.3500 & \% \text { Fuel Cld outer Plate } 6\end{array}$ surf 25 cyl $0.0 \quad 0.011 .5500 \quad \%$ Fuel Cld inner Plate 7 surf 26 cyl $0.0 \quad 0.0 \quad 11.5875 \quad \%$ Fuel Mt inner Plate 7 surf 27 cyl $0.0 \quad 0.011 .6375 \quad \%$ Fuel Mt outer Plate 7 surf 28 cyl $\begin{array}{lllll}0.0 & 0.0 & 11.6750 & \% \text { Fuel Cld outer Plate } 7\end{array}$ surf 29 cyl $0.0 \quad 0.011 .8750 \quad \%$ Fuel Cld inner Plate 8 surf 30 cyl $0.0 \quad 0.011 .9125 \quad \%$ Fuel Mt inner Plate 8 surf $31 \mathrm{cyl} \quad 0.0 \quad 0.0 \quad 11.9625 \quad \%$ Fuel Mt outer Plate 8 surf 32 cyl $0.0 \quad 0.012 .0000 \quad \%$ Fuel Cld outer Plate 8 surf 33 cyl $0.0 \quad 0.012 .2000 \quad \%$ Fuel Cld inner Plate 9 surf 34 cyl $0.0 \quad 0.0 \quad 12.2375 \quad \%$ Fuel Mt inner Plate 9 surf 35 cyl $0.0 \quad 0.0 \quad 12.2875 \quad \%$ Fuel Mt outer Plate 9 surf 36 cyl $0.0 \quad 0.012 .3250 \quad \%$ Fuel Cld outer Plate 9 surf $\begin{array}{lllll}37 & \text { cyl } & 0.0 & 0.0 & 12.5250\end{array} \%$ Fuel Cld inner Plate 10 surf 38 cyl $\begin{array}{llll}0.0 & 0.0 & 12.5625 & \% \text { Fuel Mt inner Plate } 10\end{array}$ surf 39 cyl $0.0 \quad 0.012 .6125 \quad \%$ Fuel Mt outer Plate 10 surf 40 cyl $0.0 \quad 0.012 .6500 \quad \%$ Fuel Cld outer Plate 10 surf 41 cyl $0.0 \quad 0.012 .8500 \quad \%$ Fuel Cld inner Plate 11 surf 42 cyl $0.0 \quad 0.0 \quad 12.8875 \quad \%$ Fuel Mt inner Plate 11 $\begin{array}{lllll}\text { surf } 43 \text { cyl } & 0.0 & 0.0 & 12.9375 & \% \text { Fuel Mt outer Plate } 11\end{array}$ surf 44 cyl $0.0 \quad 0.0 \quad 12.9750 \quad \%$ Fuel Cld outer Plate 11 surf 45 cyl $\begin{array}{llll}0.0 & 0.0 & 13.1750 \quad \% \text { Fuel Cld inner Plate } 12\end{array}$ surf 46 cyl $0.0 \quad 0.0 \quad 13.2125 \quad \%$ Fuel Mt inner Plate 12 surf 47 cyl $0.0 \quad 0.0 \quad 13.2625 \quad \%$ Fuel Mt outer Plate 12 surf 48 cyl $\begin{array}{lllll}0.0 & 0.0 & 13.3000 & \% \text { Fuel Cld outer Plate } 12\end{array}$

$\%$ *** vertical planes separating arcuate assemblies

surf 300 px 0.0

surf $301 \mathrm{px}-0.475$

surf 302 px 0.475

surf 303 py 0.0

surf 304 py -0.475

surf 305 py 0.475

surf 306 plane -1.01 .00 .00 .0$

surf 307 plane $-1.01 .00 .0-0.671751442$

surf 308 plane $-1.01 .00 .0 \quad 0.671751442$

surf 309 plane 1.01 .00 .00 .0

surf 310 plane $1.01 .00 .0-0.671751442$ 
surf 311 plane $1.01 .00 .0 \quad 0.671751442$

$\%$ *** Top and Bottom End Plates ***

surf $80 \mathrm{pz} 60$. \% Top of fuel meat

surf $81 \mathrm{pz}-60$. \% Bottom of fuel meat

surf $62 \mathrm{pz} 62$. \% Top of plates

surf $63 \mathrm{pz}-62$. \% Bottom of plates

surf $64 \mathrm{pz} 82$. \% Top of Top End Box

surf 65 pz -82. \% Bottom of Bottom End Box

$\%{ }^{* * *}$ Large Test Space Surfaces ***

surf $121 \mathrm{cyl} \quad 0.0 \quad 0.0 \quad 6.35 \%$ Outer surface of flux monitor holder

surf 122 cyl $0.0 \quad 0.0 \quad 6.95 \%$ Inner surface of pressure tube

surf 123 cyl $0.0 \quad 0.0 \quad 7.85 \%$ Outer surface of pressure tube

surf 124 cyl $0.0 \quad 0.07 .95 \%$ Inner surface of insulation jacket

surf $125 \mathrm{cyl} \quad 0.0 \quad 0.0 \quad 8.10 \%$ Outer surface of insulation jacket

surf 126 cyl $0.0 \quad 0.0 \quad 8.70 \%$ Inner surface of Al baffle

surf 127 cyl $0.0 \quad 0.0 \quad 9.40 \%$ Outer surface of Al baffle

surf 128 cyl $0.0 \quad 0.0 \quad 13.5 \%$ Inner surface of rack hole

$\%$ *** Small Test Space Surfaces peripheral location ***

surf 220 cyl $0.0 \quad 0.0 \quad 3.54 \%$ Outer boundary of test space

surf 221 cyl $0.0 \quad 0.0 \quad 3.70 \%$ Outer surface of flow tube

surf 222 cyl $0.0 \quad 0.0 \quad 4.00 \%$ Inner surface of pressure tube

surf 223 cyl $0.0 \quad 0.0 \quad 4.90 \%$ Outer surface of pressure tube

surf 224 cyl $0.0 \quad 0.0 \quad 5.00 \%$ Inner surface of insulation jacket

surf 225 cyl $0.0 \quad 0.0 \quad 5.30 \%$ Outer surface of insulation jacket

surf 226 cyl $0.0 \quad 0.0 \quad 5.80 \%$ Inner surface of safety rod guide tube

surf 227 cyl $0.0 \quad 0.0 \quad 6.40 \%$ Outer surface of safety rod guide tube

surf 228 cyl $0.0 \quad 0.07 .50 \%$ Inner surface of safety rod follower

surf 229 cyl $0.0 \quad 0.0 \quad 8.30 \%$ Outer surface of safety rod follower

$\%$ *** Pressure Vessel tank inner/outer boundary ***

surf 205 cyl 0.00 .051 . \%

surf 206 cyl 0.00 .055 . \%

$\%$ *** D2O tank outer boundary ***

surf 201 cyl $0.00 .0150 .0 \%$

surf $202 \mathrm{pz}-150$

surf 203 pz 150

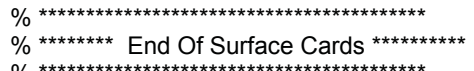

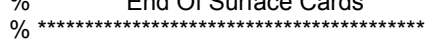

$\%$ vacuum boundary

set bc 1

$\%$ source points

$\%$ src 1 sp 1.240 .00 .0

$\%$ source entropy mesh

set entr [4 44 -150. 150. -150. 150. -50. 50. ]

$\%$ cross section library path !!!! for Fission machine !!!!

set acelib "/home/popema/SERPENT/SERPENT/data/xsdata_combined"

set declib "/home/popema/SERPENT/SERPENT/data/sss endfb7.dec"

set nfylib "/home/popema/SERPENT/SERPENT/data/sss_endfb7.nfy"

plot $3150015000-6060-6060$

mesh $3100010000-150150-150150$

set power 250.E06

set opti 1

set pop 10000100051.2

$\%$ set nbuf 10000000

$\%$------ Material Cards -

$\%$----- Fuel -----

mat fuel1 4.9186E-02 tmp 433. vol 318.7 burn 1

92235.03c 7.6963E-03

$92238.03 \mathrm{C} 3.0882 \mathrm{E}-02$

42000.03c 1.0608E-02

mat fuel2 4.9186E-02 tmp 433. vol 330.9 burn 1

92235.03c 7.6963E-03

92238.03c 3.0882E-02

42000.03 c 1.0608E-02

mat fuel3 4.9186E-02 tmp 433. vol 343.2 burn 1

92235.03c 7.6963E-03

92238.03 c $3.0882 \mathrm{E}-02$ 
42000.03c 1.0608E-02

mat fuel4 4.9186E-02 tmp 433. vol 355.4 burn 1 92235.03c 7.6963E-03

92238.03c 3.0882E-02

$42000.03 \mathrm{c} 1.0608 \mathrm{E}-02$

mat fuel5 4.9186E-02 tmp 433. vol 367.7 burn 1 92235.03 c 7.6963E-03 92238.03 c 3.0882E-02 42000.03c 1.0608E-02

mat fuel6 4.9186E-02 tmp 433. vol 379.9 burn 1 92235.03c 7.6963E-03 92238.03c 3.0882E-02 42000.03c 1.0608E-02

mat fuel7 4.9186E-02 tmp 433. vol 392.2 burn 1 92235.03c 7.6963E-03 92238.03 C 3.0882E-02 42000.03c 1.0608E-02

mat fuel8 4.9186E-02 tmp 433. vol 404.4 burn 1 92235.03c 7.6963E-03 92238.03c 3.0882E-02 42000.03c 1.0608E-02 mat fuel9 4.9186E-02 tmp 433. vol 416.7 burn 1 92235.03c 7.6963E-03 92238.03c 3.0882E-02 42000.03c 1.0608E-02

mat fuel10 4.9186E-02 tmp 433. vol 428.9 burn 1 92235.03c 7.6963E-03 92238.03 C 3.0882E-02 42000.03 c $1.0608 \mathrm{E}-02$

mat fuel11 4.9186E-02 tmp 433. vol 441.2 burn 1 92235.03c 7.6963E-03 92238.03 C 3.0882E-02 42000.03c 1.0608E-02

mat fuel12 4.9186E-02 tmp 433. vol 453.4 burn 1 92235.03c 7.6963E-03 92238.03c 3.0882E-02 42000.03c 1.0608E-02

mat fuel13 4.9186E-02 tmp 433. vol 1951.8 burn 1 92235.03 c 7.6963E-03 92238.03 C 3.0882E-02 $42000.03 \mathrm{c} 1.0608 \mathrm{E}-02$

mat fuel14 4.9186E-02 tmp 433. vol 2026.9 burn 1 92235.03c 7.6963E-03 92238.03 C 3.0882E-02 42000.03 c $1.0608 \mathrm{E}-02$

mat fuel15 4.9186E-02 tmp 433. vol 2101.9 burn 1 $92235.03 \mathrm{c}$ 7.6963E-03 $92238.03 \mathrm{C} 3.0882 \mathrm{E}-02$ 42000.03c 1.0608E-02 mat fuel16 4.9186E-02 tmp 433. vol 2177.0 burn 1 92235.03c 7.6963E-03 $92238.03 \mathrm{c} 3.0882 \mathrm{E}-02$ 42000.03 c 1.0608E-02

mat fuel17 4.9186E-02 tmp 433. vol 2252.0 burn 1 92235.03c 7.6963E-03 92238.03 C $3.0882 \mathrm{E}-02$ 42000.03c 1.0608E-02

mat fuel18 4.9186E-02 tmp 433. vol 2327.1 burn 1 92235.03c 7.6963E-03 $92238.03 \mathrm{C} 3.0882 \mathrm{E}-02$ $42000.03 \mathrm{C} 1.0608 \mathrm{E}-02$

mat fuel19 4.9186E-02 tmp 433. vol 2402.1 burn 1 92235.03c 7.6963E-03 92238.03c 3.0882E-02 $42000.03 \mathrm{c} 1.0608 \mathrm{E}-02$

mat fuel20 4.9186E-02 tmp 433. vol 2477.2 burn 1 92235.03c 7.6963E-03 92238.03 C 3.0882E-02 42000.03c 1.0608E-02

mat fuel21 4.9186E-02 tmp 433. vol 2552.2 burn 1 92235.03c 7.6963E-03 $92238.03 \mathrm{C} 3.0882 \mathrm{E}-02$ $42000.03 \mathrm{C} \quad 1.0608 \mathrm{E}-02$ mat fuel22 4.9186E-02 tmp 433. vol 2627.2 burn 1 92235.03c 7.6963E-03 92238.03c 3.0882E-02 42000.03 c 1.0608E-02

mat fuel23 4.9186E-02 tmp 433. vol 2702.3 burn 1 92235.03c 7.6963E-03 92238.03 c 3.0882E-02 
42000.03c 1.0608E-02

mat fuel24 4.9186E-02 tmp 433. vol 2777.3 burn 1

92235.03c 7.6963E-03

92238.03 c 3.0882E-02

42000.03c 1.0608E-02

$\%$----- $\mathrm{H} 2 \mathrm{O}$ Coolant ------

$\% \mathrm{~T}=70 \mathrm{C}, \mathrm{P}=2.3 \mathrm{MPa}$, rho=0.979 $\mathrm{g} / \mathrm{cc}$

mat h2o 9.818E-02 moder h2o 1001

1001.03c 6.545E-02

8016.03c 3.273E-02

$\%$----- D2O

mat d2o 9.986E-02 moder d2o 1002

1002.03 c 6.657E-02

8016.03c 3.329E-02

\% ----- Al-6061 ------

mat al6061 -2.715 \% Al-6061 density $2.715 \mathrm{~g} / \mathrm{cc}$

$14000.03 c-0.7 \% \quad 0.7 \% \mathrm{Si}$

$26000.03 \mathrm{c}-0.6 \% \quad 0.60 \% \mathrm{Fe}$

$29000.03 \mathrm{c}-0.22 \% \quad 0.22 \% \mathrm{Cu}$

$25055.03 \mathrm{c}-0.08 \% \quad 0.08 \% \mathrm{Mn}$

$12000.03 \mathrm{c}-1.0 \% \quad 1.0 \% \mathrm{Mg}$

$24000.03 c-0.2 \% \quad 0.1 \% \mathrm{Cr}$

$30000.03 c-0.08 \% \quad 0.08 \% \mathrm{Zn}$

22000.03 c $-0.03 \% \quad 0.03 \% \mathrm{Ti}$

$13027.03 c-97.09 \% \quad 97.09 \% \mathrm{Al}$

$\%$----- Al-6061 Rack / part water ---

mat AIRack 6.22E-02 \% 5\% water

14000.03c 3.87E-04\%

26000.03 C $1.67 \mathrm{E}-04 \%$

29000.03C $5.38 \mathrm{E}-05 \%$

25055.03 C 2.26E-05\%

12000.03c $6.39 \mathrm{E}-04 \%$

24000.03C 5.97E-05\%

30000.03c 1.90E-05\%

22000.03c 9.73E-06\%

13027.03C 5.59E-02\%

1001.03c 3.27E-03

8016.03c 1.64E-03

$\%$----- Beryllium ----.

$\%$ assumed $6 \%$ by volume $\mathrm{H} 2 \mathrm{O}$

mat be9 $1.2188 \mathrm{E}-01$ moder be $4009 \%$ moder be 4009.03c 1.157E-01

1001.03c 4.120E-03

8016.03c 2.060E-03

$\%$---- SS 304

$\%$ Fe-0.08C-2.0Mn-0.045P-0.03S-1.0Si-19Cr-9Ni

mat ss304 $-8.0 \quad \%$ ss $3048 \mathrm{~g} / \mathrm{cc}$

$6000.03 \mathrm{c}-0.08 \% \quad 0.08 \% \mathrm{C}$

$25055.03 \mathrm{c}-2.0 \% \quad 2.0 \% \mathrm{Mn}$

$15031.03 \mathrm{c}-0.045 \% \quad 0.045 \% \mathrm{P}$

$16000.03 c-0.03 \% \quad 0.03 \%$ S

$14000.03 \mathrm{c}-0.75 \% \quad 0.75 \% \mathrm{Si}$

$24000.03 \mathrm{c}-19 . \quad \% \quad$ 19. $\% \mathrm{Cr}$

$28000.03 \mathrm{c}-9.25 \% \quad 9.25 \% \mathrm{Ni}$

$7014.03 \mathrm{c}-0.05 \% \quad 0.05 \% \mathrm{~N}$

$26000.03 \mathrm{c}-68.795 \% \quad 68.795 \% \mathrm{Fe}$

$\%$----- Helium Coolant ----

mat helium 0.0007

2004.03c 7.0000E-04

$\%$---- Al/H2O 50/50 mix in test spaces ----

mat AlH2Omix 7.929E-02 moder h2o 1001

1001.03c 3.270E-02

8016.03 C 1.635E-02

13027.03c 3.024E-02

$\%$----- Zircalloy4 -------

mat zirc4 -6.56 tmp 398.

$40000.03 c-98.23 \% \quad 98.23 \%$ Z

$50000.03 \mathrm{c}-1.45 \% \quad 1.45 \% \mathrm{Sn}$

$26000.03 \mathrm{c}-0.21 \quad \% \quad 0.21 \% \mathrm{Fe}$

$24000.03 \mathrm{c}-0.10 \quad \% \quad 0.10 \% \mathrm{Cr}$

$72000.03 \mathrm{c}-0.01 \% \quad 0.01 \% \mathrm{Hf}$

\% ----- Top End Box -----

mix TopBox

zirc4 0.20

h2o 0.80

$\%$----- Bottom End Box -----.-

mix BotBox

zirc4 0.20

h2o 0.80

\% ----- Top plate no fuel ------- 
mix TopPlat

zirc4 0.39

h2o 0.61

$\%$---- Bottom plate no fuel ------

mix BotPlat

zirc4 0.39

h2o 0.61

$\%$ for installation on Fission

therm h2o Iwtr.01t

therm d2o hwtr.01t

therm be be.01t

dep daytot

0.10 .51 .02 .03 .55 .010 .0 15. 20. 30. 40. 50. 60. 80. 100. 120. 140. 160

$\%$ TALLIES

$\%$ Flux $1 \mathrm{MeV}$ cutoff in test zones

det CTrap dc $140 \mathrm{du} 1 \mathrm{dv}$ 432. de $1 \mathrm{MeV} \mathrm{dx}-3$. 3. $1 \mathrm{dy}-3.3 .1 \mathrm{dz}-60.60 .10$

det PTrap dc 120 du 2 dv 432. de $1 \mathrm{MeV}$ dx 31. 37. 1 dy -3. 3. $1 \mathrm{dz}-60.60 .10$

$\%$ Flux broken down into 3 .

det FTref1 dc 183 du 0 dv 192. de $1 \mathrm{MeV}$ dx 55.459 .41 dy 21.725 .71 dz -60.60 .10$ det FTref2 dc 183 du 0 dv 192. de $1 \mathrm{MeV}$ dx 73.877 .81 dy $29.433 .41 \mathrm{dz}-60.60 .10$ det FTref3 dc 183 du 0 dv 192. de $1 \mathrm{MeV}$ dx 92.396 .31 dy $37.041 .01 \mathrm{dz}-60.60 .10$ det FTref4 dc 183 du 0 dv 192. de $1 \mathrm{MeV}$ dx 110.8114 .81 dy $44.748 .71 \mathrm{dz}-60.60 .10$

ene $1 \mathrm{MeV} \quad 1$ 1.0E-15 0.625E-06 1.020 


\section{PBT - Baseline model, single-batch depletion}

$\%$ title Initial M-A Core

$\%$ Michael Pope

$\%$

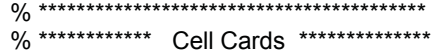

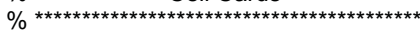

$\%^{* * *}$ Universe $1^{* * *}$ Large central Location ****

$\%$ *** Test Space ****

cell 1401 al6061 -121 \% test space

cell 1421 h2o $121-122 \quad \%$ return flow

cell 1431 ss304 $122-123 \quad \%$ pressure tube

cell 1441 helium $123-124 \quad \%$ helium annulus

cell 1451 ss304 $124-125 \quad \%$ insulation jacket

cell 1461 h2o $125-126 \quad \%$ water gap

cell 1471 al6061 $126-127 \quad \%$ baffle

cell 1481 h2o $127 \quad-1 \quad \%$ water outside baffle inside fuel plate 1

cell 1491 h2o $\quad 48-128-80 \quad 81 \%$ water outside fuel inside Pressure vessel

cell 1501 al6061 $128-129-80 \quad 81 \%$ al6061 pressure vessel next to fuel

$\begin{array}{lllll}\text { cell } 1511 & \mathrm{~d} 2 \mathrm{o} & 129 & -80 & 81\end{array} \% \mathrm{~d} 2 \mathrm{o}$ outside next to fuel

$\%{ }^{* * *}$ NNE Octant ${ }^{* * *}$

cell 10021 zirc4 1 - $-2 \quad-8081308302 \%$ Fuel Clad inner Plate 1

cell 10031 fuel1 $\quad 2 \quad-3 \quad-8081308302 \%$ Fuel Meat Plate 1

cell 10041 zirc4 $\quad 3 \quad-4 \quad-8081308302 \%$ Fuel Clad outer Plate 1

cell 10051 h2o $\quad 4 \quad-5 \quad-80 \quad 81308302 \%$ Water between Plates 1 and 2

cell 10061 zirc4 $\quad 5 \quad-6 \quad-8081308302 \%$ Fuel Clad inner Plate 2

cell 10071 fuel2 $\quad 6 \quad-7 \quad-8081308302 \%$ Fuel Meat Plate 2

$\begin{array}{llllllll}\text { cell } 1008 & 1 & \text { zirc4 } & 7 & -8 & -80 & 81308 & 302 \%\end{array}$ Fuel Clad outer Plate 2

cell $1009 \quad 1$ h2o $\quad 8 \quad-9 \quad-80 \quad 81308302 \%$ Water between Plates 2 and 3

cell 10101 zirc4 $\quad 9 \quad-10 \quad-80 \quad 81308302 \%$ Fuel Clad inner Plate 3

cell 10111 fuel3 $10-11 \quad-8081308302 \%$ Fuel Meat Plate 3

cell $1012 \quad 1$ zirc4 $\quad 11-12 \quad-80 \quad 81308302 \%$ Fuel Clad outer Plate 3

$\begin{array}{llllllll}\text { cell } 1013 & 1 & \text { h2o } & 12 & -13 & -80 & 81308 & 302 \%\end{array}$ Water between Plates 3 and 4

$\begin{array}{lllllllll}\text { cell } 1014 & 1 & \text { zirc4 } & 13 & -14 & -80 & 81 & 308 & 302\end{array} \%$ Fuel Clad inner Plate 4

cell 10151 fuel4 $\quad 14-15 \quad-80 \quad 81308302 \%$ Fuel Meat Plate 4

cell 10161 zirc4 $\quad 15-16 \quad-80 \quad 81308302 \%$ Fuel Clad outer Plate 4

cell $1017 \quad 1$ h2o $\quad 16-17-8081308302 \%$ Water between Plates 4 and 5

cell 10181 zirc4 $17 \quad-18 \quad-8081308302 \%$ Fuel Clad inner Plate 5

cell 10191 fuel5 $\quad 18-19 \quad-80 \quad 81308302 \%$ Fuel Meat Plate 5

cell 10201 zirc4 $\quad 19-20 \quad-80 \quad 81308302 \%$ Fuel Clad outer Plate 5

cell $1021 \quad 1$ h2o $20-21 \quad-80 \quad 81308302 \%$ Water between Plates 5 and 6

cell 10221 zirc4 $\quad 21 \quad-22 \quad-80 \quad 81308302 \%$ Fuel Clad inner Plate 6

cell 10231 fuel5 $\quad 22 \quad-23 \quad-80 \quad 81308302 \%$ Fuel Meat Plate 6

$\begin{array}{lllllllll}\text { cell } 1024 & 1 & \text { zirc4 } & 23 & -24 & -80 & 81308 & 302 \% & \text { Fuel Clad outer Plate } 6\end{array}$

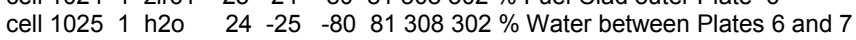

cell 10261 zirc4 $\quad 25 \quad-26 \quad-80 \quad 81308302 \%$ Fuel Clad inner Plate 7

cell 10271 fuel7 $\quad 26 \quad-27 \quad-80 \quad 81308302 \%$ Fuel Meat Plate 7

$\begin{array}{lllllllll}\text { cell } 1028 & 1 & \text { zirc4 } & 27 & -28 & -80 & 81308 & 302 & \%\end{array}$ Fuel Clad outer Plate 7

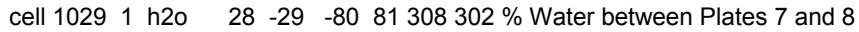

$\begin{array}{llllllll}\text { cell } 1030 & 1 & \text { zirc4 } & 29 & -30 & -80 & 81308 & 302 \% \text { Fuel Clad inner Plate } 8\end{array}$

$\begin{array}{lllllll}\text { cell } 1031 & 1 \text { fuel } 8 & 30 & -31 & -80 & 81308 & 302 \%\end{array} \%$ Fuel Meat Plate 8

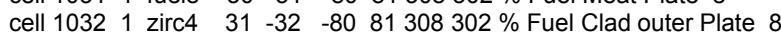

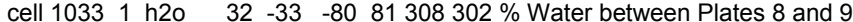

cell 10341 zirc4 $33 \quad-34 \quad-80 \quad 81308302 \%$ Fuel Clad inner Plate 9

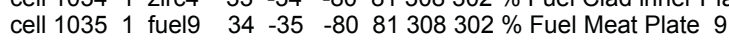

cell 10361 zirc4 $\quad 35 \quad-36 \quad-80 \quad 81308302 \%$ Fuel Clad outer Plate 9

$\begin{array}{llllllll}\text { cell } 1037 & 1 & \text { h2o } & 36 & -37 & -80 & 81308 & 302 \%\end{array} \%$ Water between Plates 9 and 10

$\begin{array}{llllllll}\text { cell } 1038 & 1 & \text { zirc4 } & 37 & -38 & -80 & 81308 & 302 \% \text { Fuel Clad inner Plate } 10\end{array}$

cell 10391 fuel10 $38 \quad-39 \quad-80 \quad 81308302 \%$ Fuel Meat Plate 10

cell $1040 \quad 1$ zirc4 $\quad 39-40 \quad-80 \quad 81308302 \%$ Fuel Clad outer Plate 10

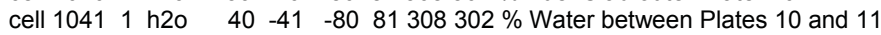

cell 10421 zirc4 $4 \begin{array}{lllll}41 & -42 & -80 & 81308302 \% & \text { Fuel Clad inner Plate } 11\end{array}$

cell 10431 fuel11 $42-43 \quad-8081308302 \%$ Fuel Meat Plate 11

cell $1044 \quad 1$ zirc4 $\quad 43 \quad-44 \quad-80 \quad 81308302 \%$ Fuel Clad outer Plate 11

cell 10451 h2o $\quad 44-45-8081308302 \%$ Water between Plates 11 and 12

cell 10461 zirc4 $45 \quad-46 \quad-8081308302 \%$ Fuel Clad inner Plate 12

cell $1047 \quad 1$ fuel12 $46 \quad-47 \quad-80 \quad 81308302 \%$ Fuel Meat Plate 12

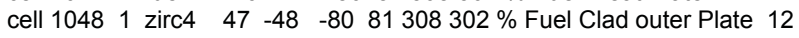

$\%$ *** ENE Octant ***

cell $1102 \quad 1$ zirc4 $\quad 1 \quad-2 \quad-80 \quad 81-307305 \%$ Fuel Clad inner Plate 1

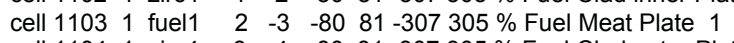

cell 11041 zirc4 $\quad 3 \quad-4 \quad-80 \quad 81-307305 \%$ Fuel Clad outer Plate 1

$\begin{array}{lllllll}\text { cell } 1105 & 1 \text { h2o } & 4 & -5 & -80 & 81-307 & 305 \% \text { Water between Plates } 1 \text { and } 2\end{array}$

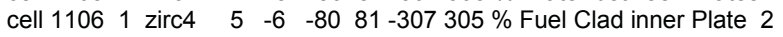


cell 11071 fuel2 $\quad 6 \quad-7 \quad-80 \quad 81-307305 \%$ Fuel Meat Plate 2

cell 11081 zirc4 $\quad 7 \quad-8 \quad-80 \quad 81-307305 \%$ Fuel Clad outer Plate 2

cell $1109 \quad 1$ h2o $\quad 8 \quad-9 \quad-80 \quad 81-307305 \%$ Water between Plates 2 and 3

cell 11101 zirc4 $\quad 9-10 \quad-80 \quad 81-307305 \%$ Fuel Clad inner Plate 3

cell 11111 fuel3 $10-11 \quad-80 \quad 81-307305 \%$ Fuel Meat Plate 3

cell $1112 \quad 1$ zirc4 $11 \quad-12 \quad-80 \quad 81-307 \quad 305 \%$ Fuel Clad outer Plate 3

$\begin{array}{lllllllll}\text { cell } 1113 & 1 & \text { h2o } & 12 & -13 & -80 & 81 & -307 & 305 \%\end{array} \%$ Water between Plates 3 and 4

cell 11141 zirc4 $13-14 \quad-80 \quad 81-307305 \%$ Fuel Clad inner Plate 4

cell 11151 fuel4 $14 \quad-15 \quad-80 \quad 81-307305 \%$ Fuel Meat Plate 4

cell $1116 \quad 1$ zirc4 $15 \quad-16 \quad-80 \quad 81-307 \quad 305 \%$ Fuel Clad outer Plate 4

$\begin{array}{lllllllll}\text { cell } 1117 & 1 & \text { h2o } & 16 & -17 & -80 & 81 & -307 & 305 \%\end{array}$ Water between Plates 4 and 5

cell 11181 zirc4 $17-18 \quad-80 \quad 81-307305 \%$ Fuel Clad inner Plate 5

cell 11191 fuel5 $18-19 \quad-80 \quad 81-307305 \%$ Fuel Meat Plate 5

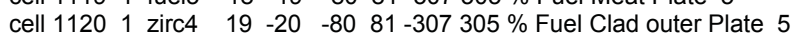

$\begin{array}{lllllllll}\text { cell } 1121 & 1 & \text { h2o } & 20 & -21 & -80 & 81 & -307 & 305 \%\end{array}$ Water between Plates 5 and 6

cell 11221 zirc4 $21 \quad-22 \quad-80 \quad 81-307305 \%$ Fuel Clad inner Plate 6

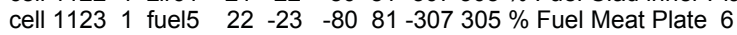

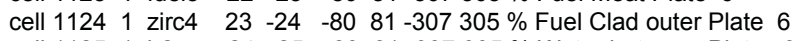

cell 11251 h2o $\quad 24-25 \quad-80 \quad 81-307305 \%$ Water between Plates 6 and 7

cell $1126 \quad 1$ zirc4 $25 \quad-26 \quad-80 \quad 81-307305 \%$ Fuel Clad inner Plate 7

$\begin{array}{llllllllll}\text { cell } 1127 & 1 \text { fuel7 } & 26 & -27 & -80 & 81 & -307 & 305 \% & \text { Fuel Meat Plate } 7\end{array}$

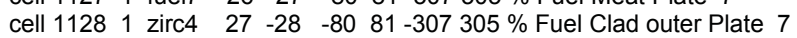

cell $1129 \quad 1$ h2o $\quad 28 \quad-29 \quad-80 \quad 81-307305 \%$ Water between Plates 7 and 8

cell 11301 zirc4 $29-30 \quad-80 \quad 81-307305 \%$ Fuel Clad inner Plate 8

$\begin{array}{llllllllll}\text { cell } 1131 & 1 & \text { fuel } 8 & 30 & -31 & -80 & 81 & -307 & 305 \% & \text { Fuel Meat Plate } 8\end{array}$

cell $1132 \quad 1$ zirc4 $31-32 \quad-80 \quad 81-307305 \%$ Fuel Clad outer Plate 8

cell 11331 h2o $\quad 32-33 \quad-80 \quad 81-307305 \%$ Water between Plates 8 and 9

cell $1134 \quad 1$ zirc4 $33 \quad-34 \quad-80 \quad 81-307305 \%$ Fuel Clad inner Plate 9

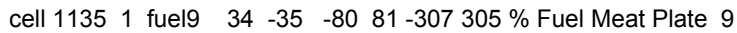

cell $1136 \quad 1$ zirc4 $35-36 \quad-80 \quad 81-307305 \%$ Fuel Clad outer Plate 9

cell $1137 \quad 1$ h2o $\quad 36-37 \quad-80 \quad 81-307305 \%$ Water between Plates 9 and 10

cell $1138 \quad 1$ zirc4 $37 \quad-38 \quad-80 \quad 81-307305 \%$ Fuel Clad inner Plate 10

cell 11391 fuel10 $38 \quad-39 \quad-80 \quad 81-307305 \%$ Fuel Meat Plate 10

cell $1140 \quad 1$ zirc4 $39-40 \quad-80 \quad 81-307305 \%$ Fuel Clad outer Plate 10

$\begin{array}{llllllllll}\text { cell } 1141 & 1 & \text { h2o } & 40 & -41 & -80 & 81 & -307 & 305 & \%\end{array}$ Water between Plates 10 and 11

cell $1142 \quad 1$ zirc4 $41-42 \quad-80 \quad 81-307305 \%$ Fuel Clad inner Plate 11

cell 11431 fuel11 $42 \quad-43 \quad-80 \quad 81-307305 \%$ Fuel Meat Plate 11

cell $1144 \quad 1$ zirc4 $43 \quad-44 \quad-80 \quad 81-307305 \%$ Fuel Clad outer Plate 11

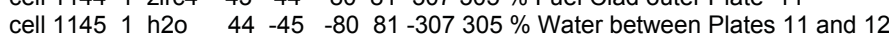

cell 11461 zirc4 $45 \quad-46 \quad-80 \quad 81-307305 \%$ Fuel Clad inner Plate 12

cell 11471 fuel12 $46 \quad-47 \quad-80 \quad 81-307305 \%$ Fuel Meat Plate 12

cell $1148 \quad 1$ zirc4 $47 \quad-48 \quad-80 \quad 81-307 \quad 305 \%$ Fuel Clad outer Plate 12

$\%{ }^{* * *}$ ESE Octant ***

cell 12021 zirc4

cell 12031 fuel 1

cell 12041 zirc4

cell $12051 \mathrm{~h} 2 \mathrm{o}$

cell 12061 zirc4

cell 12071 fuel2

cell 12081 zirc4

cell $12091 \mathrm{~h} 2 \mathrm{o}$

cell 12101 zirc4

cell 12111 fuel3

cell 12121 zirc4

cell 12131 h2o

cell 12141 zirc 4

cell 12151 fuel 4

cell $1216 \quad 1$ zirc4

cell 12171 h2o

cell 12181 zirc 4

cell 12191 fuel5

cell 12201 zirc4

cell $12211 \mathrm{~h} 2 \mathrm{o}$

cell 12221 zirc4

cell 12231 fuel5

cell 12241 zirc4

cell $12251 \mathrm{~h} 2 \mathrm{o}$

cell 12261 zirc4

cell $1227 \quad 1$ fuel7

cell 12281 zirc 4

cell $12291 \mathrm{~h} 2 \mathrm{O}$

cell $1230 \quad 1$ zirc4

cell 12311 fuel8

cell 12321 zirc4

cell 12331 h2o

cell 12341 zirc4

cell 12351 fuel9

cell 12361 zirc4

cell 12371 h2o

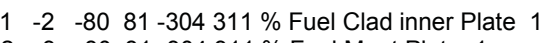

$\begin{array}{lllll}2 & -3 & -80 & 81 & -304 \\ 311 & \% & \text { Fuel Meat Plate } 1\end{array}$

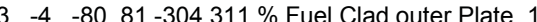

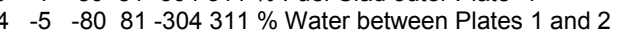

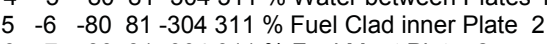

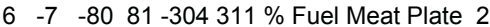

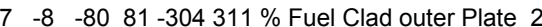

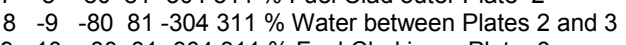

$9 \quad-10 \quad-80 \quad 81-304311 \%$ Fuel Clad inner Plate 3

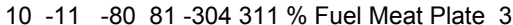

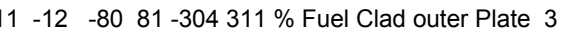

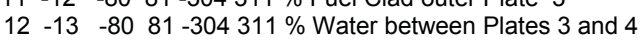

$13-14 \quad-80 \quad 81-304311 \%$ Fuel Clad inner Plate 4

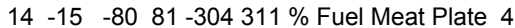

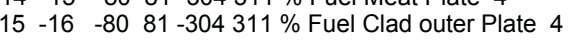

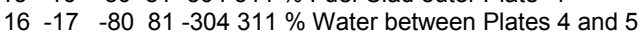

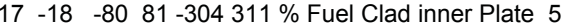

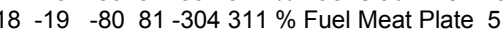

$\begin{array}{lllll}19 & -20 & -80 & 81 & -304 \\ 311 & \% & \text { Fuel Clad outer Plate } 5\end{array}$

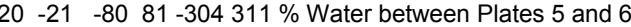

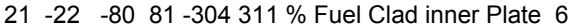

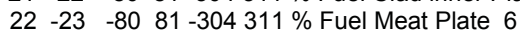

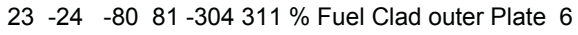

$\begin{array}{lllll}24 & -25 & -80 & 81 & -304 \\ 311 & \%\end{array}$ Water between Plates 6 and 7

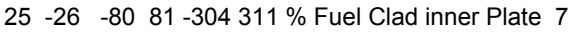

$\begin{array}{lllll}26 & -27 & -80 & 81 & -304 \\ 311 & \% & \text { Fuel Meat Plate } 7\end{array}$

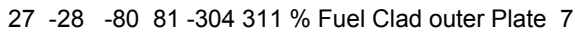

$\begin{array}{llllll}28 & -29 & -80 & 81 & -304 & 311 \%\end{array}$ Water between Plates 7 and 8

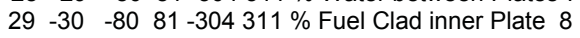

$\begin{array}{lllll}30 & -31 & -80 & 81 & -304 \\ 311 & \% & \text { Fuel Meat Plate } 8\end{array}$

$\begin{array}{llllll}31 & -32 & -80 & 81 & -304 & 311 \% \text { Fuel Clad outer Plate } 8\end{array}$

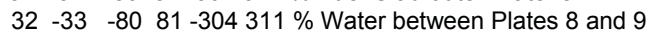

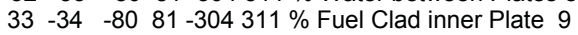

$\begin{array}{lllll}34 & -35 & -80 & 81 & -304 \\ 311 & \% & \text { Fuel Meat Plate } 9\end{array}$

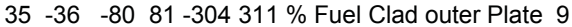

$\begin{array}{lllll}36 & -37 & -80 & 81-304 & 311 \%\end{array}$ Water between Plates 9 and 10 
cell 12381 zirc4 $37-38 \quad-80 \quad 81-304311 \%$ Fuel Clad inner Plate 10 cell 12391 fuel10 $38 \quad-39 \quad-80 \quad 81-304311 \%$ Fuel Meat Plate 10

cell $1240 \quad 1$ zirc4 $39 \quad-40 \quad-80 \quad 81-304311 \%$ Fuel Clad outer Plate 10

cell $1241 \quad 1$ h2o $40 \quad-41 \quad-80 \quad 81-304311 \%$ Water between Plates 10 and 11

cell $1242 \quad 1$ zirc4 $41 \quad-42 \quad-80 \quad 81-304311 \%$ Fuel Clad inner Plate 11

cell 12431 fuel11 $42 \quad-43 \quad-80 \quad 81-304311 \%$ Fuel Meat Plate 11

cell 12441 zirc4 $43 \quad-44 \quad-80 \quad 81-304311 \%$ Fuel Clad outer Plate 11

cell $1245 \quad 1$ h2o $44 \quad-45 \quad-80 \quad 81-304311 \%$ Water between Plates 11 and 12

cell $1246 \quad 1$ zirc4 $45 \quad-46 \quad-80 \quad 81-304311 \%$ Fuel Clad inner Plate 12

cell 12471 fuel12 $46 \quad-47 \quad-80 \quad 81-304311 \%$ Fuel Meat Plate 12

cell $1248 \quad 1$ zirc4 $47 \quad-48 \quad-80 \quad 81-304311 \%$ Fuel Clad outer Plate 12

$\%$ *** SSE Octant ***

cell 13021 zirc4

cell 13031 fuel 1

cell 13041 zirc4

cell $13051 \mathrm{~h} 2 \mathrm{o}$

cell 13061 zirc

cell 13071 fuel2

cell 13081 zirc

cell $13091 \mathrm{~h} 2 \mathrm{o}$

cell 13101 zirc4

cell 13111 fuel3

cell 13121 zirc4

cell $13131 \mathrm{~h} 2 \mathrm{o}$

cell 13141 zirc

cell 13151 fuel

cell 13161 zirc 4

cell $13171 \mathrm{~h} 20$

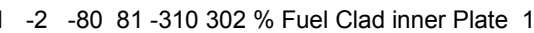

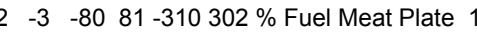

$\begin{array}{lllllll}3 & -4 & -80 & 81 & -310 & 302 \% & \text { Fuel Clad outer Plate } 1\end{array}$

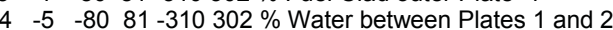

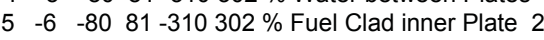

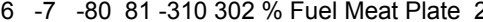

$\begin{array}{llllll}7 & -8 & -80 & 81 & -310 & 302 \%\end{array} \%$ Fuel Clad outer Plate 2

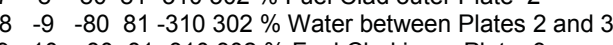

$9 \quad-10 \quad-80 \quad 81-310302 \%$ Fuel Clad inner Plate 3

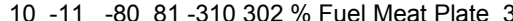

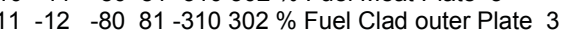

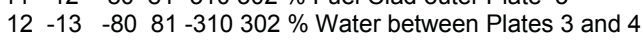

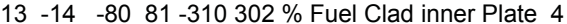

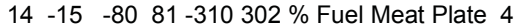

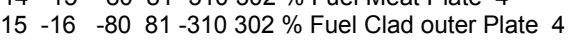

$\begin{array}{llllll}17 & -18 & -80 & 81 & -310 & 302\end{array} \%$ Fuel Clad inner Plate 5

cell 13191 fuel5 $18 \quad-19 \quad-80 \quad 81-310302 \%$ Fuel Meat Plate 5

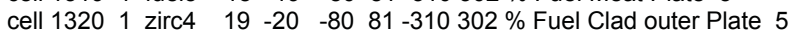

cell $1321 \quad 1$ h2o $\quad 20-21 \quad-80 \quad 81-310302 \%$ Water between Plates 5 and 6

cell $1322 \quad 1$ zirc4 $21 \quad-22 \quad-80 \quad 81-310302 \%$ Fuel Clad inner Plate 6

$\begin{array}{lllllllll}\text { cell } 1323 & 1 & \text { fuel5 } & 22 & -23 & -80 & 81 & -310 & 302\end{array} \%$ Fuel Meat Plate 6

$\begin{array}{llllllllll}\text { cell } 1324 & 1 & \text { zirc4 } & 23 & -24 & -80 & 81 & -310 & 302 & \%\end{array}$ Fuel Clad outer Plate 6

cell 13251 h2o $\quad 24 \quad-25 \quad-80 \quad 81-310302 \%$ Water between Plates 6 and 7

cell $1326 \quad 1$ zirc4 $25 \quad-26 \quad-80 \quad 81-310302 \%$ Fuel Clad inner Plate 7

$\begin{array}{lllllllll}\text { cell } 1327 & 1 & \text { fuel } 7 & 26 & -27 & -80 & 81 & -310 & 302\end{array} \%$ Fuel Meat Plate 7

cell 13281 zirc4 $27 \quad-28 \quad-80 \quad 81-310302 \%$ Fuel Clad outer Plate 7

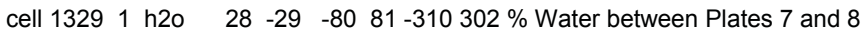

$\begin{array}{lllllllll}\text { cell } 1330 & 1 & \text { zirc4 } & 29 & -30 & -80 & 81 & -310 & 302\end{array} \%$ Fuel Clad inner Plate 8

cell 13311 fuel8 $30 \quad-31 \quad-80 \quad 81-310302 \%$ Fuel Meat Plate 8

cell $1332 \quad 1$ zirc4 $31 \quad-32 \quad-80 \quad 81-310302 \%$ Fuel Clad outer Plate 8

$\begin{array}{lllllllll}\text { cell } 1333 & 1 & \text { h2o } & 32 & -33 & -80 & 81 & -310 & 302 \% \text { Water between Plates } 8 \text { and } 9\end{array}$

cell 13341 zirc4 $33 \quad-34 \quad-80 \quad 81-310302 \%$ Fuel Clad inner Plate 9

cell 13351 fuel9 $34 \quad-35 \quad-80 \quad 81-310302 \%$ Fuel Meat Plate 9

cell $1336 \quad 1$ zirc4 $35 \quad-36 \quad-80 \quad 81-310302 \%$ Fuel Clad outer Plate 9

$\begin{array}{lllllllll}\text { cell } 1337 & 1 & \text { h2o } & 36 & -37 & -80 & 81 & -310 & 302\end{array} \%$ Water between Plates 9 and 10

cell $1338 \quad 1$ zirc4 $37 \quad-38 \quad-80 \quad 81-310302 \%$ Fuel Clad inner Plate 10

cell 13391 fuel10 $38 \quad-39 \quad-80 \quad 81-310302 \%$ Fuel Meat Plate 10

cell 13401 zirc4 $39-40 \quad-80 \quad 81-310302 \%$ Fuel Clad outer Plate 10

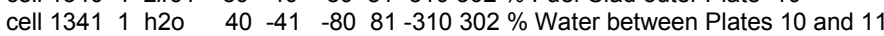

cell $1342 \quad 1$ zirc4 $41 \quad-42 \quad-80 \quad 81-310302 \%$ Fuel Clad inner Plate 11

cell 13431 fuel11 $42 \quad-43 \quad-80 \quad 81-310302 \%$ Fuel Meat Plate 11

cell $1344 \quad 1$ zirc4 $43-44 \quad-80 \quad 81-310302 \%$ Fuel Clad outer Plate 11

cell $1345 \quad 1$ h2o $\quad 44 \quad-45 \quad-80 \quad 81-310302 \%$ Water between Plates 11 and 12

cell $1346 \quad 1$ zirc4 $45 \quad-46 \quad-80 \quad 81-310302 \%$ Fuel Clad inner Plate 12

cell 13471 fuel12 $46 \quad-47 \quad-80 \quad 81-310302 \%$ Fuel Meat Plate 12

cell $1348 \quad 1$ zirc4 $47 \quad-48 \quad-80 \quad 81-310302 \%$ Fuel Clad outer Plate 12

$\%$ *** SSW Octant ***

cell 14021 zirc4 $1 \quad 1 \quad-2 \quad-80 \quad 81-301-307 \%$ Fuel Clad inner Plate 1

cell 14031 fuel1 $22 \quad-3 \quad-80 \quad 81-301-307 \%$ Fuel Meat Plate 1

cell 14041 zirc4 $\quad 3 \quad-4 \quad-80 \quad 81-301-307 \%$ Fuel Clad outer Plate 1

$\begin{array}{llllllllll}\text { cell } 1405 & 1 & \text { h2o } & 4 & -5 & -80 & 81 & -301 & -307 & \%\end{array}$ Water between Plates 1 and 2

cell 14061 zirc4 $55 \quad-6 \quad-80 \quad 81-301-307 \%$ Fuel Clad inner Plate 2

cell 14071 fuel2 $\quad 6 \quad-7 \quad-80 \quad 81-301-307 \%$ Fuel Meat Plate 2

cell 14081 zirc4 $\quad 7 \quad-8 \quad-80 \quad 81-301-307 \%$ Fuel Clad outer Plate 2

$\begin{array}{llllllllllll}\text { cell } 1409 & 1 & \text { h2o } & 8 & -9 & -80 & 81 & -301 & -307 & \% \text { Water between Plates } 2 \text { and } 3\end{array}$

cell 14101 zirc4 $9-10-80 \quad 81-301-307 \%$ Fuel Clad inner Plate 3

cell 14111 fuel3 $10-11 \quad-80 \quad 81-301-307 \%$ Fuel Meat Plate 3

cell 14121 zirc4 $11-12 \quad-80 \quad 81-301-307 \%$ Fuel Clad outer Plate 3

cell $1413 \quad 1$ h2o $\quad 12-13 \quad-80 \quad 81-301-307 \%$ Water between Plates 3 and 4

cell 14141 zirc4 $13-14 \quad-80 \quad 81-301-307 \%$ Fuel Clad inner Plate 4

cell 14151 fuel $4 \quad 14-15 \quad-80 \quad 81-301-307 \%$ Fuel Meat Plate 4

cell $1416 \quad 1$ zirc4 $15-16 \quad-80 \quad 81-301-307 \%$ Fuel Clad outer Plate 4

cell $1417 \quad 1$ h2o $\quad 16-17 \quad-80 \quad 81-301-307 \%$ Water between Plates 4 and 5

cell $1418 \quad 1$ zirc4 $\quad 17 \quad-18 \quad-80 \quad 81-301-307 \%$ Fuel Clad inner Plate 5

$\begin{array}{lllllllll}\text { cell } 1419 & 1 \text { fuel5 } & 18 & -19 & -80 & 81 & -301 & -307 \% \text { Fuel Meat Plate } 5\end{array}$ 
cell 14201 zirc4 $19-20 \quad-80 \quad 81-301-307 \%$ Fuel Clad outer Plate 5

cell $1421 \quad 1$ h2o $\quad 20-21 \quad-80 \quad 81-301-307 \%$ Water between Plates 5 and 6

cell 14221 zirc4 $21 \quad-22 \quad-80 \quad 81-301-307 \%$ Fuel Clad inner Plate 6

cell 14231 fuel5 $22 \quad-23 \quad-80 \quad 81-301-307 \%$ Fuel Meat Plate 6

cell $1424 \quad 1$ zirc4 $23-24 \quad-80 \quad 81-301-307 \%$ Fuel Clad outer Plate 6

cell $1425 \quad 1$ h2o $\quad 24 \quad-25 \quad-80 \quad 81-301-307 \%$ Water between Plates 6 and 7

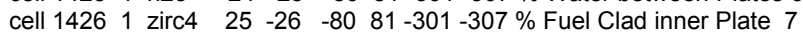

cell 14271 fuel7 $26 \quad-27 \quad-80 \quad 81-301-307 \%$ Fuel Meat Plate 7

cell $1428 \quad 1$ zirc4 $27 \quad-28 \quad-80 \quad 81-301-307 \%$ Fuel Clad outer Plate 7

$\begin{array}{llllllllll}\text { cell } 1429 & 1 & \text { h2o } & 28 & -29 & -80 & 81 & -301 & -307 & \%\end{array}$ Water between Plates 7 and 8

$\begin{array}{llllllllll}\text { cell } 1430 & 1 & \text { zirc4 } & 29 & -30 & -80 & 81 & -301 & -307 \% \text { Fuel Clad inner Plate } 8\end{array}$

cell 14311 fuel8 $30 \quad-31 \quad-80 \quad 81-301-307 \%$ Fuel Meat Plate 8

cell $1432 \quad 1$ zirc4 $31-32 \quad-80 \quad 81-301-307 \%$ Fuel Clad outer Plate 8

$\begin{array}{llllllllll}\text { cell } 1433 & 1 & \text { h2o } & 32 & -33 & -80 & 81 & -301 & -307 \% \text { Water between Plates } 8 \text { and } 9\end{array}$

cell 14341 zirc4 $33 \quad-34 \quad-80 \quad 81-301-307 \%$ Fuel Clad inner Plate 9

cell 14351 fuel9 $34 \quad-35 \quad-80 \quad 81-301-307 \%$ Fuel Meat Plate 9

cell $1436 \quad 1$ zirc4 $35-36 \quad-80 \quad 81-301-307 \%$ Fuel Clad outer Plate 9

$\begin{array}{llllllllll}\text { cell } 1437 & 1 & \text { h2o } & 36 & -37 & -80 & 81 & -301 & -307 \% \text { Water between Plates } 9 \text { and } 10\end{array}$

cell 14381 zirc4 $37 \quad-38 \quad-80 \quad 81-301-307 \%$ Fuel Clad inner Plate 10

cell 14391 fuel10 $38 \quad-39-80 \quad 81-301-307 \%$ Fuel Meat Plate 10

cell $1440 \quad 1$ zirc4 $39-40 \quad-80 \quad 81-301-307 \%$ Fuel Clad outer Plate 10

$\begin{array}{lllllllll}\text { cell } 1441 & 1 & \text { h2o } & 40 & -41 & -80 & 81 & -301 & -307\end{array} \%$ Water between Plates 10 and 11

cell 14421 zirc4 $41-42 \quad-80 \quad 81-301-307 \%$ Fuel Clad inner Plate 11

cell 14431 fuel11 $42 \quad-43 \quad-80 \quad 81-301-307 \%$ Fuel Meat Plate 11

cell $1444 \quad 1$ zirc4 $43 \quad-44 \quad-80 \quad 81-301-307 \%$ Fuel Clad outer Plate 11

cell 14451 h2o $\quad 44-45 \quad-80 \quad 81-301-307 \%$ Water between Plates 11 and 12

cell 14461 zirc4 $45 \quad-46 \quad-80 \quad 81-301-307 \%$ Fuel Clad inner Plate 12

cell $1447 \quad 1$ fuel12 $46 \quad-47 \quad-80 \quad 81-301-307 \%$ Fuel Meat Plate 12

cell $1448 \quad 1$ zirc4 $47 \quad-48 \quad-80 \quad 81-301-307 \%$ Fuel Clad outer Plate 12

$\%$ *** WSW Octant ***

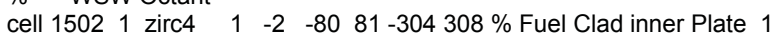

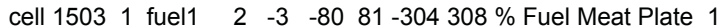

cell $1504 \quad 1$ zirc4 $\quad 3 \quad-4 \quad-80 \quad 81-304308 \%$ Fuel Clad outer Plate 1

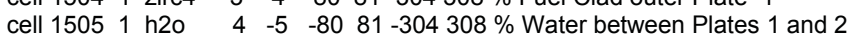

cell 15061 zirc4 $5 \quad-6 \quad-80 \quad 81-304308 \%$ Fuel Clad inner Plate 2

cell 15071 fuel2 $\quad 6 \quad-7 \quad-80 \quad 81-304308 \%$ Fuel Meat Plate 2

$\begin{array}{llllllllll}\text { cell } 1508 & 1 & \text { zirc4 } & 7 & -8 & -80 & 81 & -304 & 308 \% & \text { Fuel Clad outer Plate } 2\end{array}$

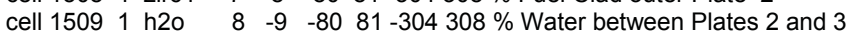

cell 15101 zirc4 $9-10 \quad-80 \quad 81-304308 \%$ Fuel Clad inner Plate 3

cell 15111 fuel3 $10-11 \quad-80 \quad 81-304308 \%$ Fuel Meat Plate 3

cell $1512 \quad 1$ zirc4 $11 \quad-12 \quad-80 \quad 81-304308 \%$ Fuel Clad outer Plate 3

cell 15131 h2o $12-13 \quad-80 \quad 81-304308 \%$ Water between Plates 3 and 4

cell $1514 \quad 1$ zirc4 $13-14 \quad-80 \quad 81-304308 \%$ Fuel Clad inner Plate 4

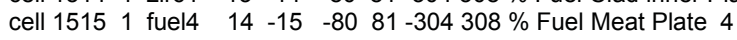

cell $1516 \quad 1$ zirc4 $15 \quad-16 \quad-80 \quad 81-304308 \%$ Fuel Clad outer Plate 4

cell $1517 \quad 1$ h2o $\quad 16 \quad-17 \quad-80 \quad 81-304308 \%$ Water between Plates 4 and 5

cell 15181 zirc4 $17-18 \quad-80 \quad 81-304308 \%$ Fuel Clad inner Plate 5

cell 15191 fuel5 $18 \quad-19 \quad-80 \quad 81-304308 \%$ Fuel Meat Plate 5

cell 15201 zirc4 $19-20 \quad-80 \quad 81-304308 \%$ Fuel Clad outer Plate 5

cell $1521 \quad 1$ h2o $\quad 20-21 \quad-80 \quad 81-304308 \%$ Water between Plates 5 and 6

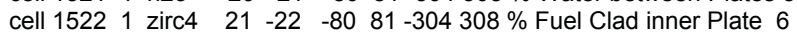

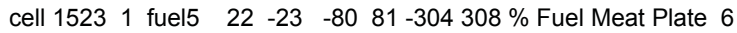

cell 15241 zirc4 $23 \quad-24 \quad-80 \quad 81-304308 \%$ Fuel Clad outer Plate 6

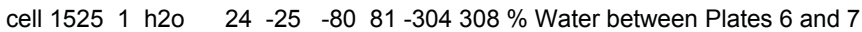

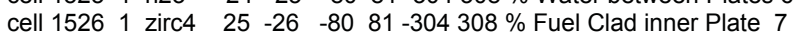

cell 15271 fuel7 $\quad 26 \quad-27 \quad-80 \quad 81-304308 \%$ Fuel Meat Plate 7

cell $1528 \quad 1$ zirc4 $27 \quad-28 \quad-80 \quad 81-304308 \%$ Fuel Clad outer Plate 7

$\begin{array}{lllllllllll}\text { cell } 1529 & 1 & \text { h2o } & 28 & -29 & -80 & 81 & -304 & 308 \% \text { Water between Plates } 7 \text { and } 8\end{array}$

cell 15301 zirc4 $29 \quad-30 \quad-80 \quad 81-304308 \%$ Fuel Clad inner Plate 8

cell 15311 fuel8 $30 \quad-31 \quad-80 \quad 81-304308 \%$ Fuel Meat Plate 8

cell $1532 \quad 1$ zirc4 $31 \quad-32 \quad-80 \quad 81-304308 \%$ Fuel Clad outer Plate 8

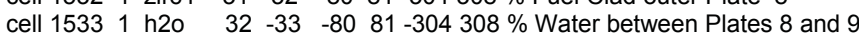

cell 15341 zirc4 $33 \quad-34 \quad-80 \quad 81-304308 \%$ Fuel Clad inner Plate 9

cell 15351 fuel9 $34 \quad-35 \quad-80 \quad 81-304308 \%$ Fuel Meat Plate 9

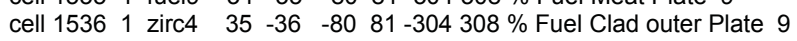

$\begin{array}{lllllllll}\text { cell } 1537 & 1 & \text { h2o } & 36 & -37 & -80 & 81 & -304 & 308\end{array} \%$ Water between Plates 9 and 10

cell 15381 zirc4 $37 \quad-38 \quad-80 \quad 81-304308 \%$ Fuel Clad inner Plate 10

cell 15391 fuel10 $38 \quad-39 \quad-80 \quad 81-304308 \%$ Fuel Meat Plate 10

cell 15401 zirc4 $39-40 \quad-80 \quad 81-304308 \%$ Fuel Clad outer Plate 10

cell $1541 \quad 1$ h2o $\quad 40-41 \quad-80 \quad 81-304308 \%$ Water between Plates 10 and 11

cell 15421 zirc4 $41 \quad-42 \quad-80 \quad 81-304308 \%$ Fuel Clad inner Plate 11

cell 15431 fuel11 $42 \quad-43 \quad-80 \quad 81-304308 \%$ Fuel Meat Plate 11

cell $1544 \quad 1$ zirc4 $43 \quad-44 \quad-80 \quad 81-304308 \%$ Fuel Clad outer Plate 11

cell $1545 \quad 1$ h2o $\quad 44-45 \quad-80 \quad 81-304308 \%$ Water between Plates 11 and 12

cell $1546 \quad 1$ zirc4 $45 \quad-46 \quad-80 \quad 81-304308 \%$ Fuel Clad inner Plate 12

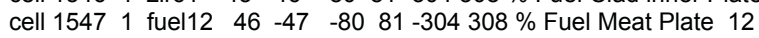

cell 15481 zirc4 $47 \quad-48 \quad-80 \quad 81-304308 \%$ Fuel Clad outer Plate 12

$\%{ }^{* * *}$ WNW Octant $* * *$ 
cell 16021 zirc4 cell 16031 fuel 1 cell 16041 zirc cell 16051 h2o cell 16061 zirc

cell 16071 fuel cell 16081 zirc4 cell 16091 h2o cell 16101 zirc4 cell 16111 fuel3 cell 16121 zirc cell 16131 h2o

cell 16141 zirc4 cell 16151 fuel 4 cell 16161 zirc4 cell 16171 h2o cell 16181 zirc4 cell 16191 fuel5 cell 16201 zirc cell 16211 h2o cell 16221 zirc4 cell 16231 fuel5 cell 16241 zirc4 cell 16251 h2o cell 16261 zirc4 cell 16271 fuel7 cell 16281 zirc4 cell 16291 h2o cell 16301 zirc4 cell 16311 fuel8 cell 16321 zirc4 cell 16331 h2o cell 16341 zirc cell 16351 fuelg cell 16361 zirc4 cell 16371 h2o cell 16381 zirc4 cell 16391 fuel

cell 16401 zirc4 cell 16411 h2o cell 16421 zirc4 cell 16431 fuel cell 16441 zirc 4 cell $16451 \mathrm{~h} 2 \mathrm{o}$ cell $1646 \quad 1$ zirc4 $45 \quad-46 \quad-80 \quad 81-310305 \%$ Fuel Clad inner Plate 12 $\begin{array}{llllll}46 & -47 & -80 & 81 & -310 & 305 \%\end{array} \%$ Fuel Meat Plate 12 cell $1648 \quad 1$ zirc4 $47 \quad-48 \quad-80 \quad 81-310305 \%$ Fuel Clad outer Plate 12

$\%{ }^{* * *}$ NNW Octant ***

cell $1702 \quad 1$ zirc4 $11-2 \quad-80 \quad 81311-301 \%$ Fuel Clad inner Plate 1 cell 17031 fuel $1 \quad 2 \quad-3 \quad-80 \quad 81311-301 \%$ Fuel Meat Plate 1 cell $1704 \quad 1$ zirc4 $3 \quad-4 \quad-80 \quad 81311-301 \%$ Fuel Clad outer Plate 1 cell $1705 \quad 1$ h2o $\quad 4 \quad-5-80 \quad 81311-301 \%$ Water between Plates 1 and 2 cell 17061 zirc4 5 -6 $-80 \quad 81311-301 \%$ Fuel Clad inner Plate 2 cell 17071 fuel2 $\quad 6 \quad-7 \quad-80 \quad 81311-301 \%$ Fuel Meat Plate 2 cell $1708 \quad 1$ zirc4 $\quad 7 \quad-8 \quad-80 \quad 81311-301 \%$ Fuel Clad outer Plate 2 cell 17091 h2o $\quad 8 \quad-9 \quad-80 \quad 81311-301 \%$ Water between Plates 2 and 3 cell 17101 zirc4 $9-10-80 \quad 81311-301 \%$ Fuel Clad inner Plate 3 cell 17111 fuel3 $10-11-8081311-301 \%$ Fuel Meat Plate 3 cell 17121 zirc4 $11-12-80 \quad 81311-301 \%$ Fuel Clad outer Plate 3 cell $1713 \quad 1$ h2o $\quad 12-13 \quad-80 \quad 81311-301 \%$ Water between Plates 3 and 4 cell 17141 zirc4 $13-14-8081311-301 \%$ Fuel Clad inner Plate 4 cell 17151 fuel4 $14-15-80 \quad 81311-301 \%$ Fuel Meat Plate 4 cell 17161 zirc4 $15-16 \quad-80 \quad 81311-301 \%$ Fuel Clad outer Plate 4 cell $1717 \quad 1$ h2o $\quad 16 \quad-17 \quad-80 \quad 81311-301 \%$ Water between Plates 4 and 5 cell $1718 \quad 1$ zirc4 $17 \quad-18 \quad-80 \quad 81311-301 \%$ Fuel Clad inner Plate 5 cell 17191 fuel5 $18-19-80 \quad 81311-301 \%$ Fuel Meat Plate 5 cell 17201 zirc4 $19-20 \quad-80 \quad 81311-301 \%$ Fuel Clad outer Plate 5 $\begin{array}{llllllllll}\text { cell } 1721 & 1 \text { h2o } & 20 & -21 & -80 & 81 & 311 & -301 \% \text { Water between Plates } 5 \text { and } 6\end{array}$ cell $1722 \quad 1$ zirc4 $21 \quad-22 \quad-80 \quad 81311-301 \%$ Fuel Clad inner Plate 6 cell 17231 fuel5 $22 \quad-23-80 \quad 81311-301 \%$ Fuel Meat Plate 6 cell $1724 \quad 1$ zirc4 $23-24 \quad-80 \quad 81311-301 \%$ Fuel Clad outer Plate 6 $\begin{array}{lllllllll}\text { cell } 1725 & 1 & \text { h2o } & 24 & -25 & -80 & 81 & 311 & -301 \% \text { Water between Plates } 6 \text { and } 7\end{array}$ cell 17261 zirc4 $25 \quad-26 \quad-80 \quad 81311-301 \%$ Fuel Clad inner Plate 7 cell 17271 fuel7 $26 \quad-27 \quad-80 \quad 81311-301 \%$ Fuel Meat Plate 7 cell $1728 \quad 1$ zirc4 $27 \quad-28 \quad-80 \quad 81311-301 \%$ Fuel Clad outer Plate 7 $\begin{array}{lllllllllll}\text { cell } 1729 & 1 & \text { h2o } & 28 & -29 & -80 & 81 & 311 & -301 \% \text { Water between Plates } 7 \text { and } 8\end{array}$ cell 17301 zirc4 $29-30-80 \quad 81311-301 \%$ Fuel Clad inner Plate 8 cell 17311 fuel8 $30 \quad-31 \quad-80 \quad 81311-301 \%$ Fuel Meat Plate 8 cell $1732 \quad 1$ zirc4 $31 \quad-32 \quad-80 \quad 81311-301 \%$ Fuel Clad outer Plate 8 
cell 17331 h2o $32-33-80 \quad 81311-301 \%$ Water between Plates 8 and 9 cell 17341 zirc4 $33 \quad-34-80 \quad 81311-301 \%$ Fuel Clad inner Plate 9

cell 17351 fuel9 $34 \quad-35-80 \quad 81311-301 \%$ Fuel Meat Plate 9

$\begin{array}{lllllllllll}\text { cell } 1736 & 1 & \text { zirc4 } & 35 & -36 & -80 & 81 & 311 & -301 \% \text { Fuel Clad outer Plate } 9\end{array}$

cell $1737 \quad 1$ h2o $\quad 36-37 \quad-80 \quad 81311-301 \%$ Water between Plates 9 and 10

cell 17381 zirc4 $37-38-80 \quad 81311-301 \%$ Fuel Clad inner Plate 10

cell 17391 fuel10 $38 \quad-39-80 \quad 81311-301 \%$ Fuel Meat Plate 10

cell 17401 zirc4 $39-40 \quad-80 \quad 81311-301 \%$ Fuel Clad outer Plate 10

cell $1741 \quad 1 \mathrm{~h} 2 \mathrm{o} \quad 40 \quad-41-80 \quad 81311-301 \%$ Water between Plates 10 and 11

cell 17421 zirc4 $41 \quad-42-80 \quad 81311-301 \%$ Fuel Clad inner Plate 11

cell 17431 fuel11 $42 \quad-43 \quad-80 \quad 81311-301 \%$ Fuel Meat Plate 11

cell $1744 \quad 1$ zirc4 $43 \quad-44 \quad-80 \quad 81311-301 \%$ Fuel Clad outer Plate 11

cell $1745 \quad 1$ h2o $\quad 44-45 \quad-80 \quad 81311-301 \%$ Water between Plates 11 and 12

cell $1746 \quad 1$ zirc4 $45 \quad-46 \quad-80 \quad 81311-301 \%$ Fuel Clad inner Plate 12

cell 17471 fuel12 $46 \quad-47-80 \quad 81311-301 \%$ Fuel Meat Plate 12

cell 17481 zirc4 $47 \quad-48$-80 $81311-301 \%$ Fuel Clad outer Plate 12

$\%$ Other materials above and below fuel assembly

cell $\begin{array}{llllll}77 & 1 & \mathrm{~h} 2 \mathrm{o} & 1 & -128 & 64\end{array} \%$ water above fuel assembly

$\begin{array}{lllllll}\text { cell } & 78 & 1 & \mathrm{~h} 2 \mathrm{o} & 1 & -128 & -65\end{array} \%$ water below fuel assembly

$\%$ Upper and lower plates without fuel homogenized

cell 1851 TopPlat $1-128 \quad 80-62 \quad \%$ upper end plates

cell 1861 BotPlat $1-128-8163 \quad \%$ lower end plates

$\%$ Upper and lower end boxes homogenized

cell $187 \quad 1$ TopBox $1-128 \quad 62-64 \%$ upper end box

cell 1881 BotBox $1-128 \quad-63 \quad 65 \%$ lower end box

cell $79 \quad 1$ al6061 $128-129 \quad 80 \quad \%$ pressure vessel above fuel assembly cell 801 al6061 $128-129 \quad-81 \quad \%$ pressure vessel below fuel assembly

$\begin{array}{llllll}\text { cell } & 81 & 1 & \text { d2o } & 129 & 80\end{array} \%$ d2o outside PV above fuel assembly

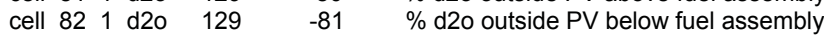

$\%$ Side plates (fused adjacent plates

cell 901 zirc4 1 -48 $301-302 \quad 303-80 \quad 81 \%$ N sideplates

cell 911 zirc4 1 -48 $307-308 \quad 303-80 \quad 81 \%$ NE sideplates

cell 921 zirc4 $1-48304-305 \quad 300-80 \quad 81 \%$ E sideplates

cell 931 zirc4 1 -48 $310-311-303-80 \quad 81 \%$ SE sideplates

cell 941 zirc4 $1-48301-302-303-80 \quad 81 \%$ S sideplates

cell 951 zirc4 $1-48307-308-303-80 \quad 81 \%$ SW sideplates

cell 961 zirc4 1 -48 $304-305-300-80 \quad 81 \%$ W sideplates

cell 971 zirc4 $1-48 \quad 310-311 \quad 303-80 \quad 81 \%$ NW sideplates

$\%$ *** Outer Test Location *** Universe 2 ****

cell 1202 al6061 $-220 \quad \%$ test space

cell 1212 ss304 $220-221 \%$ flow tube

cell 1222 h2o $221-222 \%$ return flow

cell 1232 ss304 $222-223 \%$ pressure tube

cell 1242 helium $223-224 \%$ helium annulus

cell 1252 ss304 $224-225 \%$ insulation jacket

cell 1262 h2o $225-226 \%$ water gap

cell 1272 al6061 $226-227 \%$ guide tube

cell 1282 h2o $227-228 \%$ water

cell 1292 al6061 $228-229 \%$ safety rod follower

cell 1302 h2o $229-126 \quad \%$ water just inside baffle

cell 1312 al6061 $126-127 \%$ baffle

cell 1322 h2o $127 \quad-1 \quad \%$ water just inside fuel plate 1

cell 1332 h2o $\quad 48-128-8081 \%$ water outside fuel inside Pressure vesse

cell 1342 al6061 $128-129-8081 \%$ al6061 pressure vessel next to fuel

$\begin{array}{llllll}\text { cell } 1352 & \text { d2o } & 129 & -80 & 81 & \% \\ \text { d2o outside next to fuel }\end{array}$

$\%$ *** NNE Octant ***

cell 20022 zirc4 $\quad 1 \quad-2 \quad-80 \quad 81308302 \%$ Fuel Clad inner Plate 1

cell 20032 fuel13 $2 \quad-3 \quad-8081308302 \%$ Fuel Meat Plate 1

cell 20042 zirc4 $3 \quad-4 \quad-8081308302 \%$ Fuel Clad outer Plate 1

cell 20052 h2o $\quad 4 \quad-5 \quad-80 \quad 81308302 \%$ Water between Plates 1 and 2

cell 20062 zirc4 $\quad 5 \quad-6 \quad-80 \quad 81308302 \%$ Fuel Clad inner Plate 2

cell 20072 fuel14 $6 \begin{array}{lllll}-7 & -80 & 81308 & 302 \% & \text { Fuel Meat Plate } 2\end{array}$

cell $2008 \quad 2$ zirc4 $\quad 7 \quad-8 \quad-80 \quad 81308302 \%$ Fuel Clad outer Plate 2

cell 20092 h2o $\quad 8 \quad-9 \quad-80 \quad 81308302 \%$ Water between Plates 2 and 3

cell 20102 zirc4 $9-10 \quad-80 \quad 81308302 \%$ Fuel Clad inner Plate 3

cell 20112 fuel15 $10-11 \quad-8081308302 \%$ Fuel Meat Plate 3

cell 20122 zirc4 $11 \quad-12 \quad-8081308302 \%$ Fuel Clad outer Plate 3

cell 20132 h2o $12 \quad-13 \quad-80 \quad 81308302 \%$ Water between Plates 3 and 4

cell 20142 zirc4 $13-14 \quad-8081308302 \%$ Fuel Clad inner Plate 4

cell 20152 fuel16 $14 \quad-15 \quad-80 \quad 81308302 \%$ Fuel Meat Plate 4

cell 20162 zirc4 $15-16 \quad-80 \quad 81308302 \%$ Fuel Clad outer Plate 4

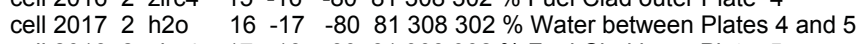

cell 20182 zirc4 $17 \quad-18 \quad-80 \quad 81308302 \%$ Fuel Clad inner Plate 5

cell 20192 fuel17 $18 \quad-19 \quad-80 \quad 81308302 \%$ Fuel Meat Plate 5

cell 20202 zirc4 $19 \quad-20 \quad-80 \quad 81308302 \%$ Fuel Clad outer Plate 5 
cell 20212 h2o $\quad 20-21 \quad-80 \quad 81308302 \%$ Water between Plates 5 and 6 cell 20222 zirc4 $21 \quad-22 \quad-80 \quad 81308302 \%$ Fuel Clad inner Plate 6 cell 20232 fuel18 $22 \quad-23 \quad-80 \quad 81308302 \%$ Fuel Meat Plate 6 cell 20242 zirc4 $23 \quad-24 \quad-80 \quad 81308302 \%$ Fuel Clad outer Plate 6 cell 20252 h2o $\quad 24 \quad-25 \quad-80 \quad 81308302 \%$ Water between Plates 6 and 7 cell $2026 \quad 2$ zirc4 $25 \quad-26 \quad-80 \quad 81308302 \%$ Fuel Clad inner Plate 7

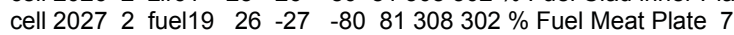
cell $2028 \quad 2$ zirc4 $27 \quad-28 \quad-80 \quad 81308302 \%$ Fuel Clad outer Plate 7 cell 20292 h2o $\quad 28-29 \quad-80 \quad 81308302 \%$ Water between Plates 7 and 8 cell 20302 zirc4 $29 \quad-30 \quad-80 \quad 81308302 \%$ Fuel Clad inner Plate 8 cell 20312 fuel20 $30 \quad-31 \quad-80 \quad 81308302 \%$ Fuel Meat Plate 8 cell 20322 zirc4 $31 \quad-32 \quad-80 \quad 81308302 \%$ Fuel Clad outer Plate 8 cell 20332 h2o $\quad 32 \quad-33 \quad-80 \quad 81308302 \%$ Water between Plates 8 and 9 cell 20342 zirc4 $33 \quad-34 \quad-80 \quad 81308302 \%$ Fuel Clad inner Plate 9 cell 20352 fuel21 $34 \quad-35 \quad-80 \quad 81308302 \%$ Fuel Meat Plate 9 cell $2036 \quad 2$ zirc4 $35 \quad-36 \quad-80 \quad 81308302 \%$ Fuel Clad outer Plate 9 cell 20372 h2o $\quad 36-37 \quad-80 \quad 81308302 \%$ Water between Plates 9 and 10 cell 20382 zirc4 $37 \quad-38 \quad-80 \quad 81308302 \%$ Fuel Clad inner Plate 10 cell 20392 fuel22 $38 \quad-39 \quad-80 \quad 81308302 \%$ Fuel Meat Plate 10 cell 20402 zirc4 $39-40 \quad-80 \quad 81308302 \%$ Fuel Clad outer Plate 10 $\begin{array}{llllllll}\text { cell } 2041 & 2 & \text { h2o } & 40 & -41 & -80 & 81308 & 302 \%\end{array}$ Water between Plates 10 and 11 cell 20422 zirc4 $41-42 \quad-80 \quad 81308302 \%$ Fuel Clad inner Plate 11 cell 20432 fuel23 $42 \quad-43 \quad-8081308302 \%$ Fuel Meat Plate 11 cell 20442 zirc4 $43 \quad-44 \quad-8081308302 \%$ Fuel Clad outer Plate 11

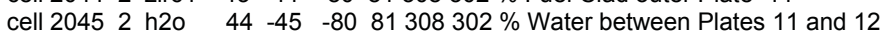
cell 20462 zirc4 $45 \quad-46 \quad-8081308302 \%$ Fuel Clad inner Plate 12 cell 20472 fuel24 $46 \quad-47 \quad-80 \quad 81308302 \%$ Fuel Meat Plate 12 cell 20482 zirc4 $47 \quad-48 \quad-80 \quad 81308302 \%$ Fuel Clad outer Plate 12

$\%$ *** ENE Octant ***

cell $2102 \quad 2$ zirc4 $1-2 \quad-80 \quad 81-307305 \%$ Fuel Clad inner Plate 1

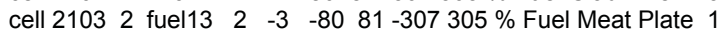

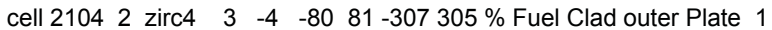
cell 21052 h2o $\quad 4 \quad-5 \quad-80 \quad 81-307305 \%$ Water between Plates 1 and 2 cell 21062 zirc4 $5 \quad-6 \quad-80 \quad 81-307305 \%$ Fuel Clad inner Plate 2

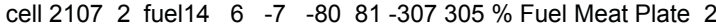
cell 21082 zirc4 $\quad 7 \quad-8 \quad-80 \quad 81-307305 \%$ Fuel Clad outer Plate 2

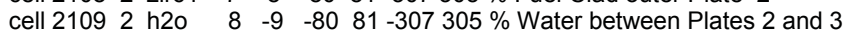

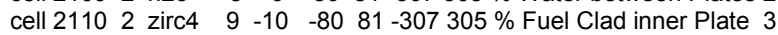
cell 21112 fuel15 $10 \quad-11 \quad-80 \quad 81-307305 \%$ Fuel Meat Plate 3

cell $2112 \quad 2$ zirc4 $11 \quad-12 \quad-80 \quad 81-307305 \%$ Fuel Clad outer Plate 3 $\begin{array}{lllllllll}\text { cell } 2113 & 2 & \text { h2o } & 12 & -13 & -80 & 81 & -307 & 305\end{array} \%$ Water between Plates 3 and 4 cell 21142 zirc4 $13-14 \quad-80 \quad 81-307305 \%$ Fuel Clad inner Plate 4 cell 21152 fuel16 $14 \quad-15 \quad-80 \quad 81-307305 \%$ Fuel Meat Plate 4 cell 21162 zirc4 $15 \quad-16 \quad-80 \quad 81-307305 \%$ Fuel Clad outer Plate 4 $\begin{array}{lllllllll}\text { cell } 2117 & 2 & \text { h2o } & 16 & -17 & -80 & 81 & -307 & 305\end{array} \%$ Water between Plates 4 and 5 cell 21182 zirc4 $17 \quad-18 \quad-80 \quad 81-307305 \%$ Fuel Clad inner Plate 5 cell 21192 fuel17 $18-19-80 \quad 81-307305 \%$ Fuel Meat Plate 5 cell 21202 zirc4 $19-20 \quad-80 \quad 81-307305 \%$ Fuel Clad outer Plate 5 $\begin{array}{lllllllll}\text { cell } 2121 & 2 & \text { h2o } & 20 & -21 & -80 & 81 & -307 & 305 \%\end{array} \%$ Water between Plates 5 and 6 cell $2122 \quad 2$ zirc4 $21 \quad-22 \quad-80 \quad 81-307305 \%$ Fuel Clad inner Plate 6

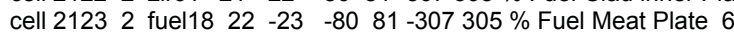
cell 21242 zirc4 $23 \quad-24 \quad-80 \quad 81-307305 \%$ Fuel Clad outer Plate 6 cell 21252 h2o $\quad 24 \quad-25 \quad-80 \quad 81-307305 \%$ Water between Plates 6 and 7 cell 21262 zirc4 $25 \quad-26 \quad-80 \quad 81-307305 \%$ Fuel Clad inner Plate 7

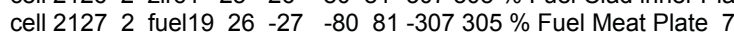
cell 21282 zirc4 $27 \quad-28 \quad-80 \quad 81-307305 \%$ Fuel Clad outer Plate 7

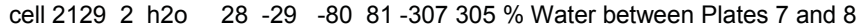
cell 21302 zirc4 $29 \quad-30 \quad-80 \quad 81-307305 \%$ Fuel Clad inner Plate 8 cell 21312 fuel20 $30 \quad-31 \quad-80 \quad 81-307305 \%$ Fuel Meat Plate 8 cell $2132 \quad 2$ zirc4 $31 \quad-32 \quad-80 \quad 81-307305 \%$ Fuel Clad outer Plate 8 $\begin{array}{lllllllll}\text { cell } 2133 & 2 & \text { h2o } & 32 & -33 & -80 & 81 & -307 & 305 \% \text { Water between Plates } 8 \text { and } 9\end{array}$ cell 21342 zirc4 $33 \quad-34 \quad-80 \quad 81-307305 \%$ Fuel Clad inner Plate 9 cell 21352 fuel21 $34 \quad-35 \quad-80 \quad 81-307305 \%$ Fuel Meat Plate 9

cell 21362 zirc4 $35 \quad-36 \quad-80 \quad 81-307305 \%$ Fuel Clad outer Plate 9

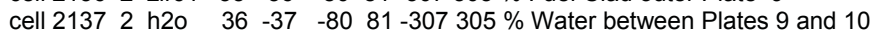
cell 21382 zirc4 $37 \quad-38 \quad-80 \quad 81-307305 \%$ Fuel Clad inner Plate 10 cell 21392 fuel22 $38 \quad-39 \quad-80 \quad 81-307305 \%$ Fuel Meat Plate 10 cell 21402 zirc4 $39 \quad-40 \quad-80 \quad 81-307 \quad 305 \%$ Fuel Clad outer Plate 10

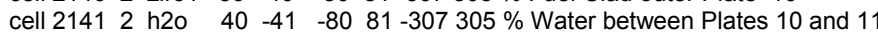
cell 21422 zirc4 $41 \quad-42 \quad-80 \quad 81-307305 \%$ Fuel Clad inner Plate 11 cell 21432 fuel23 $42 \quad-43 \quad-80 \quad 81-307305 \%$ Fuel Meat Plate 11 cell 21442 zirc4 $43 \quad-44 \quad-80 \quad 81-307305 \%$ Fuel Clad outer Plate 11 cell 21452 h2o $\quad 44-45 \quad-80 \quad 81-307305 \%$ Water between Plates 11 and 12 cell 21462 zirc4 $45 \quad-46 \quad-80 \quad 81-307305 \%$ Fuel Clad inner Plate 12 cell 21472 fuel24 $46 \quad-47 \quad-80 \quad 81-307305 \%$ Fuel Meat Plate 12 cell $2148 \quad 2$ zirc4 $47 \quad-48 \quad-80 \quad 81-307 \quad 305 \%$ Fuel Clad outer Plate 12

$\%$ *** ESE Octant ***

cell 22022 zirc4 $1 \quad-2 \quad-80 \quad 81-304311 \%$ Fuel Clad inner Plate 1 
cell 22032 fuel13 $2 \quad-3 \quad-80 \quad 81-304311 \%$ Fuel Meat Plate 1 cell 22042 zirc4 $3 \quad-4 \quad-80 \quad 81-304311 \%$ Fuel Clad outer Plate 1 cell 22052 h2o $\quad 4 \quad-5 \quad-80 \quad 81-304311 \%$ Water between Plates 1 and 2 cell 22062 zirc4 $5 \quad 5 \quad-6 \quad-80 \quad 81-304311 \%$ Fuel Clad inner Plate 2 cell 22072 fuel14 $6 \begin{array}{llllll}6 & -7 & -80 & 81 & -304 & 311 \%\end{array}$ cell $2208 \quad 2$ zirc4 $\quad 7 \quad-8 \quad-80 \quad 81-304311 \%$ Fuel Clad outer Plate 2

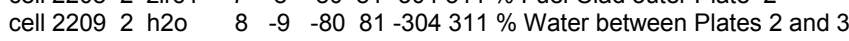
cell 22102 zirc4 $9-10 \quad-80 \quad 81-304311 \%$ Fuel Clad inner Plate 3 cell 22112 fuel15 $10-11 \quad-80 \quad 81-304311 \%$ Fuel Meat Plate 3 cell 22122 zirc4 $11-12 \quad-80 \quad 81-304311 \%$ Fuel Clad outer Plate 3

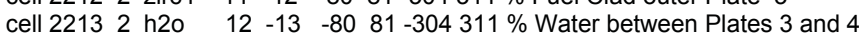
cell 22142 zirc4 $13-14 \quad-80 \quad 81-304311 \%$ Fuel Clad inner Plate 4 cell 22152 fuel16 $14 \quad-15 \quad-80 \quad 81-304311 \%$ Fuel Meat Plate 4 cell 22162 zirc4 $15-16 \quad-80 \quad 81-304311 \%$ Fuel Clad outer Plate 4 cell 22172 h2o $\quad 16 \quad-17 \quad-80 \quad 81-304311 \%$ Water between Plates 4 and 5 cell 22182 zirc4 $17-18 \quad-80 \quad 81-304311 \%$ Fuel Clad inner Plate 5 cell 22192 fuel17 $18-19 \quad-80 \quad 81-304311 \%$ Fuel Meat Plate 5 cell 22202 zirc4 $19-20 \quad-80 \quad 81-304311 \%$ Fuel Clad outer Plate 5 cell 22212 h2o $\quad 20-21 \quad-80 \quad 81-304311 \%$ Water between Plates 5 and 6 cell $2222 \quad 2$ zirc4 $21 \quad-22 \quad-80 \quad 81-304311 \%$ Fuel Clad inner Plate 6 cell 22232 fuel18 $22 \quad-23 \quad-80 \quad 81-304311 \%$ Fuel Meat Plate 6 cell 22242 zirc4 $23 \quad-24 \quad-80 \quad 81-304311 \%$ Fuel Clad outer Plate 6 cell 22252 h2o $\quad 24 \quad-25 \quad-80 \quad 81-304311 \%$ Water between Plates 6 and 7 cell 22262 zirc4 $25 \quad-26 \quad-80 \quad 81-304311 \%$ Fuel Clad inner Plate 7

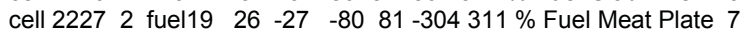
cell 22282 zirc4 $27 \quad-28 \quad-80 \quad 81-304311 \%$ Fuel Clad outer Plate 7 $\begin{array}{lllllllll}\text { cell } 2229 & 2 & \text { h2o } & 28 & -29 & -80 & 81 & -304 & 311 \% \\ \text { Water between Plates } 7 \text { and } 8\end{array}$ cell 22302 zirc4 $29-30 \quad-80 \quad 81-304311 \%$ Fuel Clad inner Plate 8 cell 22312 fuel20 $30 \quad-31 \quad-80 \quad 81-304311 \%$ Fuel Meat Plate 8 cell $2232 \quad 2$ zirc4 $31 \quad-32 \quad-80 \quad 81-304311 \%$ Fuel Clad outer Plate 8 cell 22332 h2o $\quad 32-33 \quad-80 \quad 81-304311 \%$ Water between Plates 8 and 9 cell 22342 zirc4 $33-34 \quad-80 \quad 81-304311 \%$ Fuel Clad inner Plate 9 cell 22352 fuel21 $34 \quad-35 \quad-80 \quad 81-304311 \%$ Fuel Meat Plate 9 cell 22362 zirc4 $35-36 \quad-80 \quad 81-304311 \%$ Fuel Clad outer Plate 9 $\begin{array}{llllllllll}\text { cell } 2237 & 2 & \text { h2o } & 36 & -37 & -80 & 81 & -304 & 311 \% \text { Water between Plates } 9 \text { and } 10\end{array}$ cell 22382 zirc4 $37-38 \quad-80 \quad 81-304311 \%$ Fuel Clad inner Plate 10 cell 22392 fuel22 $38 \quad-39 \quad-80 \quad 81-304311 \%$ Fuel Meat Plate 10 cell 22402 zirc4 $39-40 \quad-80 \quad 81-304311 \%$ Fuel Clad outer Plate 10

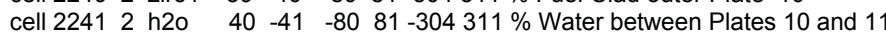
cell 22422 zirc4 $41 \quad-42 \quad-80 \quad 81-304311 \%$ Fuel Clad inner Plate 11 cell 22432 fuel23 $42 \quad-43 \quad-80 \quad 81-304311 \%$ Fuel Meat Plate 11 cell 22442 zirc4 $43 \quad-44 \quad-80 \quad 81-304311 \%$ Fuel Clad outer Plate 11 cell 22452 h2o $\quad 44-45 \quad-80 \quad 81-304311 \%$ Water between Plates 11 and 12 cell 22462 zirc4 $45-46 \quad-80 \quad 81-304311 \%$ Fuel Clad inner Plate 12 cell 22472 fuel24 $46 \quad-47 \quad-80 \quad 81-304311 \%$ Fuel Meat Plate 12 cell 22482 zirc4 $47 \quad-48 \quad-80 \quad 81-304311 \%$ Fuel Clad outer Plate 12

$\%$ *** SSE Octant ***

cell 23022 zirc4 $1 \quad-2 \quad-80 \quad 81-310302 \%$ Fuel Clad inner Plate 1

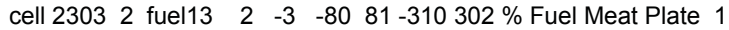
cell 23042 zirc4 $\quad 3 \quad-4 \quad-80 \quad 81-310302 \%$ Fuel Clad outer Plate 1

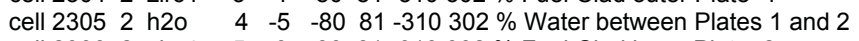
cell $2306 \quad 2$ zirc4 $\quad 5 \quad-6 \quad-80 \quad 81-310302 \%$ Fuel Clad inner Plate 2 cell $2307 \quad 2$ fuel14 $6 \begin{array}{llllll}-7 & -80 & 81 & -310 & 302 \% & \text { Fuel Meat Plate } 2\end{array}$ cell $2308 \quad 2$ zirc4 $\quad 7 \quad-8 \quad-80 \quad 81-310302 \%$ Fuel Clad outer Plate 2

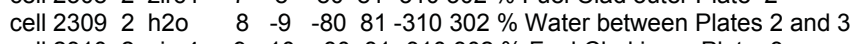
cell 23102 zirc4 $9-10 \quad-80 \quad 81-310302 \%$ Fuel Clad inner Plate 3 cell 23112 fuel15 $10-11 \quad-80 \quad 81-310302 \%$ Fuel Meat Plate 3 cell 23122 zirc4 $11-12 \quad-80 \quad 81-310302 \%$ Fuel Clad outer Plate 3

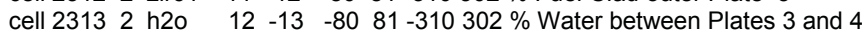
cell 23142 zirc4 $13-14 \quad-80 \quad 81-310302 \%$ Fuel Clad inner Plate 4 cell 23152 fuel16 $14 \quad-15 \quad-80 \quad 81-310302 \%$ Fuel Meat Plate 4 cell 23162 zirc4 $15-16 \quad-80 \quad 81-310302 \%$ Fuel Clad outer Plate 4 cell $2317 \quad 2$ h2o $\quad 16 \quad-17 \quad-80 \quad 81-310302 \%$ Water between Plates 4 and 5 cell 23182 zirc4 $17 \quad-18 \quad-80 \quad 81-310302 \%$ Fuel Clad inner Plate 5 cell 23192 fuel17 18 -19 $-80 \quad 81-310302 \%$ Fuel Meat Plate 5 cell 23202 zirc4 $19 \quad-20 \quad-80 \quad 81-310302 \%$ Fuel Clad outer Plate 5

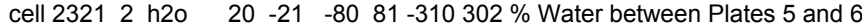
cell 23222 zirc4 $21 \quad-22 \quad-80 \quad 81-310302 \%$ Fuel Clad inner Plate 6

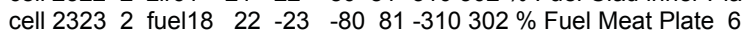
cell 23242 zirc4 $23-24 \quad-80 \quad 81-310302 \%$ Fuel Clad outer Plate 6 cell $2325 \quad 2$ h2o $\quad 24 \quad-25 \quad-80 \quad 81-310302 \%$ Water between Plates 6 and 7 cell 23262 zirc4 $25 \quad-26 \quad-80 \quad 81-310302 \%$ Fuel Clad inner Plate 7

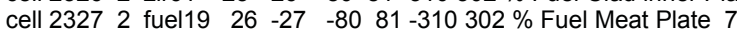
cell 23282 zirc4 $27 \quad-28 \quad-80 \quad 81-310302 \%$ Fuel Clad outer Plate 7

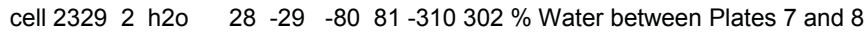
cell 23302 zirc4 $29-30 \quad-80 \quad 81-310302 \%$ Fuel Clad inner Plate 8 $\begin{array}{lllllll}\text { cell } 23312 \text { fuel20 } & 30 & -31 & -80 & 81 & -310 & 302 \%\end{array} \%$ Fuel Meat Plate 8 cell 23322 zirc4 $31 \quad-32 \quad-80 \quad 81-310302 \%$ Fuel Clad outer Plate 8 $\begin{array}{lllllllll}\text { cell } 2333 & 2 & \text { h2o } & 32 & -33 & -80 & 81 & -310 & 302\end{array} \%$ Water between Plates 8 and 9 
cell 23342 zirc4 $33 \quad-34 \quad-80 \quad 81-310302 \%$ Fuel Clad inner Plate 9 cell 23352 fuel21 $34 \quad-35 \quad-80 \quad 81-310302 \%$ Fuel Meat Plate 9 cell 23362 zirc4 $35 \quad-36 \quad-80 \quad 81-310302 \%$ Fuel Clad outer Plate 9

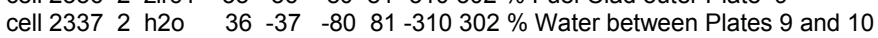
cell $2338 \quad 2$ zirc4 $37 \quad-38 \quad-80 \quad 81-310302 \%$ Fuel Clad inner Plate 10 cell 23392 fuel22 $38 \quad-39 \quad-80 \quad 81-310302 \%$ Fuel Meat Plate 10 cell 23402 zirc4 $39 \quad-40 \quad-80 \quad 81-310302 \%$ Fuel Clad outer Plate 10

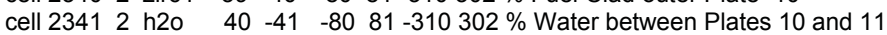
cell 23422 zirc4 $41 \quad-42 \quad-80 \quad 81-310302 \%$ Fuel Clad inner Plate 11 cell 23432 fuel23 $42 \quad-43 \quad-80 \quad 81-310302 \%$ Fuel Meat Plate 11 cell 23442 zirc4 $43 \quad-44 \quad-80 \quad 81-310302 \%$ Fuel Clad outer Plate 11 cell 23452 h2o $\quad 44-45 \quad-80 \quad 81-310302 \%$ Water between Plates 11 and 12 cell 23462 zirc4 $45 \quad-46 \quad-80 \quad 81-310302 \%$ Fuel Clad inner Plate 12 cell 23472 fuel24 $46 \quad-47 \quad-80 \quad 81-310302 \%$ Fuel Meat Plate 12 cell $2348 \quad 2$ zirc4 $47 \quad-48 \quad-80 \quad 81-310302 \%$ Fuel Clad outer Plate 12

$\%$ *** SSW Octant ***

cell 24022 zirc4 1 -2 $-80 \quad 81-301-307 \%$ Fuel Clad inner Plate 1 cell 24032 fuel13 2 -3 $-80 \quad 81-301-307 \%$ Fuel Meat Plate 1

cell 24042 zirc4 $3 \quad-4-80 \quad 81-301-307 \%$ Fuel Clad outer Plate 1

cell 24052 h2o $\quad 4 \quad-5-80 \quad 81-301-307 \%$ Water between Plates 1 and 2

cell 24062 zirc4 $5 \quad-6 \quad-80 \quad 81-301-307 \%$ Fuel Clad inner Plate 2

cell 24072 fuel14 $6 \begin{array}{lllllll}-7 & -80 & 81 & -301 & -307 \% & \text { Fuel Meat Plate } 2\end{array}$

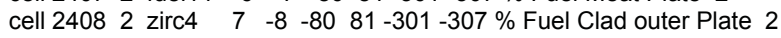

cell 24092 h2o $\quad 8 \quad-9-80 \quad 81-301-307 \%$ Water between Plates 2 and 3

cell 24102 zirc4 $9-10-80 \quad 81-301-307 \%$ Fuel Clad inner Plate 3

cell 24112 fuel15 $10-11-8081-301-307 \%$ Fuel Meat Plate 3

cell 24122 zirc4 $11-12 \quad-80 \quad 81-301-307 \%$ Fuel Clad outer Plate 3

cell $2413 \quad 2$ h2o 12 -13 $-80 \quad 81-301-307 \%$ Water between Plates 3 and 4

cell 24142 zirc4 $13-14-80 \quad 81-301-307 \%$ Fuel Clad inner Plate 4

cell 24152 fuel16 $14-15-80 \quad 81-301-307 \%$ Fuel Meat Plate 4

cell 24162 zirc4 $15-16-80 \quad 81-301-307 \%$ Fuel Clad outer Plate 4

cell 24172 h2o $\quad 16 \quad-17-80 \quad 81-301-307 \%$ Water between Plates 4 and 5

cell 24182 zirc4 $17-18-80 \quad 81-301-307 \%$ Fuel Clad inner Plate 5

cell 24192 fuel17 $18-19-80 \quad 81-301-307 \%$ Fuel Meat Plate 5

cell 24202 zirc4 $19-20-80 \quad 81-301-307 \%$ Fuel Clad outer Plate 5

cell 24212 h2o $\quad 20-21-80 \quad 81-301-307 \%$ Water between Plates 5 and 6

cell 24222 zirc4 $21-22-80 \quad 81-301-307 \%$ Fuel Clad inner Plate 6

cell 24232 fuel18 $22 \quad-23 \quad-80 \quad 81-301-307 \%$ Fuel Meat Plate 6

cell 24242 zirc4 $23-24-80 \quad 81-301-307 \%$ Fuel Clad outer Plate 6

$\begin{array}{lllllllll}\text { cell } 2425 & 2 & \text { h2o } & 24 & -25 & -80 & 81 & -301 & -307 \% \text { Water between Plates } 6 \text { and } 7\end{array}$

cell 24262 zirc4 $25 \quad-26-80 \quad 81-301-307 \%$ Fuel Clad inner Plate 7

cell 24272 fuel19 $26 \quad-27-80 \quad 81-301-307 \%$ Fuel Meat Plate 7

cell 24282 zirc4 $27 \quad-28 \quad-80 \quad 81-301-307 \%$ Fuel Clad outer Plate 7

$\begin{array}{llllllllll}\text { cell } 2429 & 2 & \text { h2o } & 28 & -29 & -80 & 81 & -301 & -307 \% \text { Water between Plates } 7 \text { and } 8\end{array}$

cell 24302 zirc4 $29-30-80 \quad 81-301-307 \%$ Fuel Clad inner Plate 8

cell 24312 fuel20 $30 \quad-31 \quad-80 \quad 81-301-307 \%$ Fuel Meat Plate 8

cell 24322 zirc4 $31-32 \quad-80 \quad 81-301-307 \%$ Fuel Clad outer Plate 8

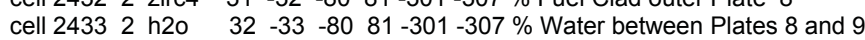

cell 24342 zirc4 $33-34-80 \quad 81-301-307 \%$ Fuel Clad inner Plate 9

cell 24352 fuel21 $34-35-80 \quad 81-301-307 \%$ Fuel Meat Plate 9

cell 24362 zirc4 $35-36-80 \quad 81-301-307 \%$ Fuel Clad outer Plate 9

$\begin{array}{lllllllll}\text { cell } 2437 & 2 & \text { h2o } & 36 & -37 & -80 & 81 & -301 & -307 \% \text { Water between Plates } 9 \text { and } 10\end{array}$

cell 24382 zirc4 $37 \quad-38-80 \quad 81-301-307 \%$ Fuel Clad inner Plate 10

cell 24392 fuel22 $38-39-80 \quad 81-301-307 \%$ Fuel Meat Plate 10

cell 24402 zirc4 $39-40-80 \quad 81-301-307 \%$ Fuel Clad outer Plate 10

cell 24412 h2o $\quad 40-41-80 \quad 81-301-307 \%$ Water between Plates 10 and 11

cell 24422 zirc4 $41-42-80 \quad 81-301-307 \%$ Fuel Clad inner Plate 11

cell 24432 fuel23 $42-43-80 \quad 81-301-307 \%$ Fuel Meat Plate 11

cell 24442 zirc4 $43 \quad-44-80 \quad 81-301-307 \%$ Fuel Clad outer Plate 11

cell 24452 h2o $\quad 44-45-80 \quad 81-301-307 \%$ Water between Plates 11 and 12

cell 24462 zirc4 $45 \quad-46-80 \quad 81-301-307 \%$ Fuel Clad inner Plate 12

cell 24472 fuel24 $46 \quad-47-80 \quad 81-301-307 \%$ Fuel Meat Plate 12

cell 24482 zirc4 $47 \quad-48$-80 81 -301-307\% Fuel Clad outer Plate 12

$\%$ *** WSW Octant ***

cell 25022 zirc4 $\quad 1 \quad-2 \quad-80 \quad 81-304308 \%$ Fuel Clad inner Plate 1

cell 25032 fuel13 $22 \quad-3 \quad-80 \quad 81-304308 \%$ Fuel Meat Plate 1

cell 25042 zirc4 $\quad 3 \quad-4 \quad-80 \quad 81-304308 \%$ Fuel Clad outer Plate 1

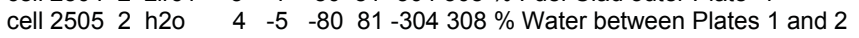

cell 25062 zirc4 5 - 5 - 5 -80 $81-304308 \%$ Fuel Clad inner Plate 2

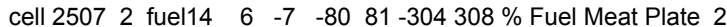

cell 25082 zirc4 $\quad 7 \quad-8 \quad-80 \quad 81-304308 \%$ Fuel Clad outer Plate 2

cell 25092 h2o $\quad 8 \quad-9 \quad-80 \quad 81-304308 \%$ Water between Plates 2 and 3

cell 25102 zirc4 $9-10 \quad-80 \quad 81-304308 \%$ Fuel Clad inner Plate 3

cell 25112 fuel15 $10-11 \quad-80 \quad 81-304308 \%$ Fuel Meat Plate 3

cell 25122 zirc4 $11-12 \quad-80 \quad 81-304308 \%$ Fuel Clad outer Plate 3

cell 25132 h2o $\quad 12-13 \quad-80 \quad 81-304308 \%$ Water between Plates 3 and 4

cell 25142 zirc4 $13-14 \quad-80 \quad 81-304308 \%$ Fuel Clad inner Plate 4

cell 25152 fuel16 $14 \quad-15 \quad-80 \quad 81-304308 \%$ Fuel Meat Plate 4 
cell 25162 zirc4 $15-16 \quad-80 \quad 81-304308 \%$ Fuel Clad outer Plate 4 cell $2517 \quad 2$ h2o $\quad 16 \quad-17 \quad-80 \quad 81-304308 \%$ Water between Plates 4 and 5 cell 25182 zirc4 $17-18 \quad-80 \quad 81-304308 \%$ Fuel Clad inner Plate 5 cell 25192 fuel17 18 -19 $\quad-80 \quad 81-304308 \%$ Fuel Meat Plate 5 cell 25202 zirc4 $19-20 \quad-80 \quad 81-304308 \%$ Fuel Clad outer Plate 5 $\begin{array}{llllllllll}\text { cell } 2521 & 2 & \text { h2o } & 20 & -21 & -80 & 81 & -304 & 308 \% & \text { Water between Plates } 5 \text { and } 6\end{array}$ cell 25222 zirc4 $21 \quad-22 \quad-80 \quad 81-304308 \%$ Fuel Clad inner Plate 6 cell 25232 fuel18 $22 \quad-23 \quad-80 \quad 81-304308 \%$ Fuel Meat Plate 6 cell 25242 zirc4 $23 \quad-24 \quad-80 \quad 81-304308 \%$ Fuel Clad outer Plate 6 cell 25252 h2o $\quad 24 \quad-25 \quad-80 \quad 81-304308 \%$ Water between Plates 6 and 7 cell 25262 zirc4 $25 \quad-26 \quad-80 \quad 81-304308 \%$ Fuel Clad inner Plate 7 cell 25272 fuel19 $26 \quad-27 \quad-80 \quad 81-304308 \%$ Fuel Meat Plate 7 cell 25282 zirc4 $27 \quad-28 \quad-80 \quad 81-304308 \%$ Fuel Clad outer Plate 7

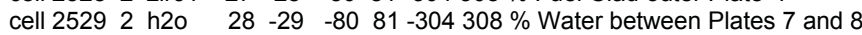
cell 25302 zirc4 $29 \quad-30 \quad-80 \quad 81-304308 \%$ Fuel Clad inner Plate 8 cell 25312 fuel20 $30 \quad-31 \quad-80 \quad 81-304308 \%$ Fuel Meat Plate 8 cell 25322 zirc4 $31 \quad-32 \quad-80 \quad 81-304308 \%$ Fuel Clad outer Plate 8

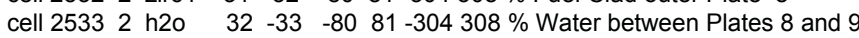
cell 25342 zirc4 $33 \quad-34 \quad-80 \quad 81-304308 \%$ Fuel Clad inner Plate 9 cell 25352 fuel21 $34 \quad-35 \quad-80 \quad 81-304308 \%$ Fuel Meat Plate 9 cell 25362 zirc4 $35-36 \quad-80 \quad 81-304308 \%$ Fuel Clad outer Plate 9 cell $2537 \quad 2$ h2o $\quad 36-37 \quad-80 \quad 81-304308 \%$ Water between Plates 9 and 10 cell 25382 zirc4 $37 \quad-38 \quad-80 \quad 81-304308 \%$ Fuel Clad inner Plate 10 cell 25392 fuel22 $38 \quad-39 \quad-80 \quad 81-304308 \%$ Fuel Meat Plate 10 cell 25402 zirc4 $39-40 \quad-80 \quad 81-304308 \%$ Fuel Clad outer Plate 10 cell $2541 \quad 2$ h2o $\quad 40-41 \quad-80 \quad 81-304308 \%$ Water between Plates 10 and 11 cell 25422 zirc4 $41 \quad-42 \quad-80 \quad 81-304308 \%$ Fuel Clad inner Plate 11 cell 25432 fuel23 $42 \quad-43 \quad-80 \quad 81-304308 \%$ Fuel Meat Plate 11 cell 25442 zirc4 $43 \quad-44 \quad-80 \quad 81-304308 \%$ Fuel Clad outer Plate 11 cell $2545 \quad 2$ h2o $\quad 44-45 \quad-80 \quad 81-304308 \%$ Water between Plates 11 and 12 cell 25462 zirc4 $45 \quad-46 \quad-80 \quad 81-304308 \%$ Fuel Clad inner Plate 12 cell 25472 fuel24 $46 \quad-47 \quad-80 \quad 81-304308 \%$ Fuel Meat Plate 12 cell 25482 zirc4 $47 \quad-48 \quad-80 \quad 81-304308 \%$ Fuel Clad outer Plate 12

$\%$ *** WNW Octant ***

cell 26022 zirc4 $1 \quad-2 \quad-80 \quad 81-310305 \%$ Fuel Clad inner Plate 1 cell 26032 fuel13 $22 \quad-3 \quad-80 \quad 81-310305 \%$ Fuel Meat Plate 1 cell 26042 zirc4 $3 \quad-4 \quad-80 \quad 81-310305 \%$ Fuel Clad outer Plate 1

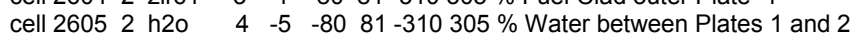
cell 26062 zirc4 5 -6 $-6 \quad-80 \quad 81-310305 \%$ Fuel Clad inner Plate 2

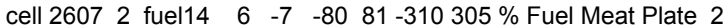
cell 26082 zirc4 $\quad 7 \quad-8 \quad-80 \quad 81-310305 \%$ Fuel Clad outer Plate 2 cell 26092 h2o $\quad 8 \quad-9 \quad-80 \quad 81-310305 \%$ Water between Plates 2 and 3 cell 26102 zirc4 $9-10 \quad-80 \quad 81-310305 \%$ Fuel Clad inner Plate 3 cell 26112 fuel15 $10-11 \quad-80 \quad 81-310305 \%$ Fuel Meat Plate 3 cell 26122 zirc4 $11 \quad-12 \quad-80 \quad 81-310305 \%$ Fuel Clad outer Plate 3 cell 26132 h2o $\quad 12 \quad-13 \quad-80 \quad 81-310305 \%$ Water between Plates 3 and 4 cell 26142 zirc4 $13-14 \quad-80 \quad 81-310305 \%$ Fuel Clad inner Plate 4 cell 26152 fuel16 $14-15 \quad-80 \quad 81-310305 \%$ Fuel Meat Plate 4 cell 26162 zirc4 $15-16 \quad-80 \quad 81-310305 \%$ Fuel Clad outer Plate 4 cell $2617 \quad 2$ h2o $\quad 16-17 \quad-80 \quad 81-310305 \%$ Water between Plates 4 and 5 cell 26182 zirc4 $17 \quad-18 \quad-80 \quad 81-310305 \%$ Fuel Clad inner Plate 5 cell 26192 fuel17 $18-19 \quad-80 \quad 81-310305 \%$ Fuel Meat Plate 5 cell 26202 zirc4 $19-20 \quad-80 \quad 81-310305 \%$ Fuel Clad outer Plate 5

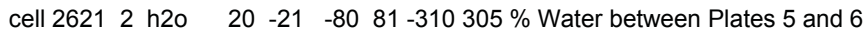
cell 26222 zirc4 $21 \quad-22 \quad-80 \quad 81-310305 \%$ Fuel Clad inner Plate 6 cell 26232 fuel18 $22 \quad-23 \quad-80 \quad 81-310305 \%$ Fuel Meat Plate 6 cell 26242 zirc4 $23 \quad-24 \quad-80 \quad 81-310305 \%$ Fuel Clad outer Plate 6

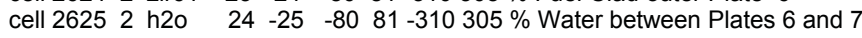
cell $2626 \quad 2$ zirc4 $25 \quad-26 \quad-80 \quad 81-310305 \%$ Fuel Clad inner Plate 7 cell 26272 fuel19 $26 \quad-27 \quad-80 \quad 81-310305 \%$ Fuel Meat Plate 7 cell 26282 zirc4 $27 \quad-28 \quad-80 \quad 81-310305 \%$ Fuel Clad outer Plate 7 $\begin{array}{lllllllll}\text { cell } 2629 & 2 & \text { h2o } & 28 & -29 & -80 & 81 & -310 & 305 \%\end{array}$ Water between Plates 7 and 8 cell 26302 zirc4 $29 \quad-30 \quad-80 \quad 81-310305 \%$ Fuel Clad inner Plate 8 cell 26312 fuel20 $30 \quad-31 \quad-80 \quad 81-310305 \%$ Fuel Meat Plate 8 cell 26322 zirc4 $31 \quad-32 \quad-80 \quad 81-310305 \%$ Fuel Clad outer Plate 8

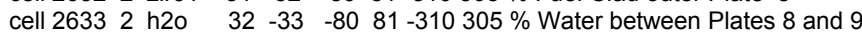
cell 26342 zirc4 $33-34 \quad-80 \quad 81-310305 \%$ Fuel Clad inner Plate 9 cell 26352 fuel21 $34-35 \quad-80 \quad 81-310305 \%$ Fuel Meat Plate 9 cell 26362 zirc4 $35-36 \quad-80 \quad 81-310305 \%$ Fuel Clad outer Plate 9 cell $2637 \quad 2$ h2o $\quad 36 \quad-37 \quad-80 \quad 81-310305 \%$ Water between Plates 9 and 10 cell 26382 zirc4 $37-38 \quad-80 \quad 81-310305 \%$ Fuel Clad inner Plate 10 cell 26392 fuel22 $38 \quad-39 \quad-80 \quad 81-310305 \%$ Fuel Meat Plate 10 cell $2640 \quad 2$ zirc4 $39 \quad-40 \quad-80 \quad 81-310305 \%$ Fuel Clad outer Plate 10 cell 26412 h2o $\quad 40 \quad-41 \quad-80 \quad 81-310305 \%$ Water between Plates 10 and 11 cell 26422 zirc4 $41 \quad-42 \quad-80 \quad 81-310305 \%$ Fuel Clad inner Plate 11 cell 26432 fuel23 $42 \quad-43 \quad-80 \quad 81-310305 \%$ Fuel Meat Plate 11 cell 26442 zirc4 $43 \quad-44 \quad-80 \quad 81-310305 \%$ Fuel Clad outer Plate 11 cell 26452 h2o $\quad 44-45 \quad-80 \quad 81-310305 \%$ Water between Plates 11 and 12 cell 26462 zirc4 $45 \quad-46 \quad-80 \quad 81-310305 \%$ Fuel Clad inner Plate 12 
cell 26472 fuel24 $46 \quad-47 \quad-80 \quad 81-310305 \%$ Fuel Meat Plate 12 cell 26482 zirc4 $47 \quad-48 \quad-80 \quad 81-310305 \%$ Fuel Clad outer Plate 12

$\%$ *** NNW Octant ***

cell 27022 zirc4 1 -2 $-80 \quad 81311-301 \%$ Fuel Clad inner Plate 1

cell 27032 fuel13 $2 \quad-3 \quad-80 \quad 81311-301 \%$ Fuel Meat Plate 1

cell 27042 zirc4 $3 \quad-4-80 \quad 81311-301 \%$ Fuel Clad outer Plate 1

cell 27052 h2o $\quad 4 \quad-5 \quad-80 \quad 81311-301 \%$ Water between Plates 1 and 2

cell 27062 zirc4 5 - 6 -80 $81311-301 \%$ Fuel Clad inner Plate 2

cell $2707 \quad 2$ fuel14 $6 \begin{array}{llllll}-8 & -80 & 81311-301 \% \text { Fuel Meat Plate } 2\end{array}$

cell 27082 zirc4 $7 \quad-8 \quad-8081311-301 \%$ Fuel Clad outer Plate 2

cell 27092 h2o $\quad 8 \quad-9 \quad-80 \quad 81311-301 \%$ Water between Plates 2 and 3

cell 27102 zirc4 $9-10-80 \quad 81311-301 \%$ Fuel Clad inner Plate 3

cell 27112 fuel15 $10-11-8081311-301 \%$ Fuel Meat Plate 3

cell 27122 zirc4 11 -12 $-80 \quad 81311-301 \%$ Fuel Clad outer Plate 3

cell 27132 h2o $12 \quad-13 \quad-80 \quad 81311-301 \%$ Water between Plates 3 and 4

cell 27142 zirc4 $13-14-8081311-301 \%$ Fuel Clad inner Plate 4

cell 27152 fuel16 $14-15-80 \quad 81311-301 \%$ Fuel Meat Plate 4

cell 27162 zirc4 15 -16 $-8081311-301 \%$ Fuel Clad outer Plate 4

cell $2717 \quad 2$ h2o $\quad 16 \quad-17 \quad-80 \quad 81311-301 \%$ Water between Plates 4 and 5

cell $2718 \quad 2$ zirc4 $17 \quad-18 \quad-80 \quad 81311-301 \%$ Fuel Clad inner Plate 5

cell 27192 fuel17 $18-19-80 \quad 81311-301 \%$ Fuel Meat Plate 5

cell 27202 zirc4 $19-20-80 \quad 81311-301 \%$ Fuel Clad outer Plate 5

cell 27212 h2o $20 \quad-21-80 \quad 81311-301 \%$ Water between Plates 5 and 6

cell 27222 zirc4 $21 \quad-22 \quad-80 \quad 81311-301 \%$ Fuel Clad inner Plate 6

cell 27232 fuel18 $22 \quad-23-80 \quad 81311-301 \%$ Fuel Meat Plate 6

cell 27242 zirc4 $23 \quad-24 \quad-80 \quad 81311-301 \%$ Fuel Clad outer Plate 6

cell 27252 h2o $24-25 \quad-80 \quad 81311-301 \%$ Water between Plates 6 and 7

cell 27262 zirc4 $25 \quad-26 \quad-80 \quad 81311-301 \%$ Fuel Clad inner Plate 7

cell 27272 fuel19 $26 \quad-27-80 \quad 81311-301 \%$ Fuel Meat Plate 7

cell $2728 \quad 2$ zirc4 $27 \quad-28 \quad-80 \quad 81311-301 \%$ Fuel Clad outer Plate 7

$\begin{array}{llllllllll}\text { cell } 2729 & 2 & \text { h2o } & 28 & -29 & -80 & 81 & 311 & -301 \% \text { Water between Plates } 7 \text { and } 8\end{array}$

cell 27302 zirc4 $29-30-80 \quad 81311-301 \%$ Fuel Clad inner Plate 8

cell 27312 fuel20 $30 \quad-31-80 \quad 81311-301 \%$ Fuel Meat Plate 8

cell 27322 zirc4 $31 \quad-32-80 \quad 81311-301 \%$ Fuel Clad outer Plate 8

cell 27332 h2o $32 \quad-33-80 \quad 81311-301 \%$ Water between Plates 8 and 9

cell 27342 zirc4 $33-34-80 \quad 81311-301 \%$ Fuel Clad inner Plate 9

cell 27352 fuel21 $34 \quad-35-80 \quad 81311-301 \%$ Fuel Meat Plate 9

cell 27362 zirc4 $35 \quad-36 \quad-80 \quad 81311-301 \%$ Fuel Clad outer Plate 9

cell $2737 \quad 2$ h2o $\quad 36-37 \quad-80 \quad 81311-301 \%$ Water between Plates 9 and 10

cell 27382 zirc4 $37 \quad-38-80 \quad 81311-301 \%$ Fuel Clad inner Plate 10

cell 27392 fuel22 38 -39 $-80 \quad 81311-301 \%$ Fuel Meat Plate 10

cell 27402 zirc4 $39-40-80 \quad 81311-301 \%$ Fuel Clad outer Plate 10

cell 27412 h2o $\quad 40-41 \quad-80 \quad 81311-301 \%$ Water between Plates 10 and 11

cell 27422 zirc4 $41 \quad-42 \quad-80 \quad 81311-301 \%$ Fuel Clad inner Plate 11

cell 27432 fuel23 $42 \quad-43-80 \quad 81311-301 \%$ Fuel Meat Plate 11

cell 27442 zirc4 $43 \quad-44 \quad-80 \quad 81311-301 \%$ Fuel Clad outer Plate 11

cell 27452 h2o $\quad 44 \quad-45 \quad-80 \quad 81311-301 \%$ Water between Plates 11 and 12

cell 27462 zirc4 $45 \quad-46 \quad-80 \quad 81311-301 \%$ Fuel Clad inner Plate 12

cell 27472 fuel24 46 -47 $-8081311-301 \%$ Fuel Meat Plate 12

cell 27482 zirc4 $47 \quad-48$-80 $81311-301 \%$ Fuel Clad outer Plate 12

$\%$ Other materials above and below fuel assembly

cell $\begin{array}{llllll}577 & 2 & \text { h2o } & 1 & -128 & 64\end{array} \%$ water above fuel assembly

$\begin{array}{llllll}\text { cell } 578 & 2 & \text { h2o } & 1 & -128 & -65\end{array} \%$ water below fuel assembly

$\%$ Upper and lower plates without fuel homogenized

cell 5852 TopPlat $1-128 \quad 80-62 \quad \%$ upper end plates

cell 5862 BotPlat $1-128-8163 \quad \%$ lower end plates

$\%$ Upper and lower end boxes homogenized

cell 5872 TopBox $1-128 \quad 62-64 \quad \%$ upper end box

cell 5882 BotBox $1-128 \quad-6365 \quad \%$ lower end box

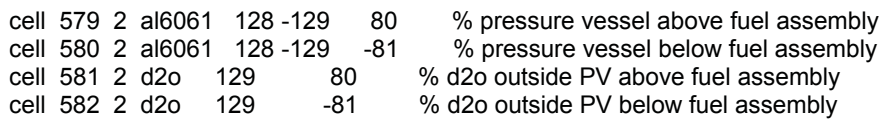

$\%$ Side plates (fused adjacent plates

cell 5902 zirc4 $1-48301-302 \quad 303-80 \quad 81 \% \mathrm{~N}$ sideplates

cell 5912 zirc4 $1-48307-308 \quad 303-80 \quad 81 \%$ NE sideplates

cell 5922 zirc4 1 -48 $304-305 \quad 300-80 \quad 81 \%$ E sideplates

cell 5932 zirc4 $1-48310-311-303-80 \quad 81 \%$ SE sideplates

cell 5942 zirc4 $1-48 \quad 301-302-303-80 \quad 81 \%$ S sideplates

cell 5952 zirc4 $1-48307-308-303-80 \quad 81 \%$ SW sideplates

cell 5962 zirc4 1 -48 $304-305-300-8081 \%$ W sideplates

cell 5972 zirc4 $1-48310-311303-80 \quad 81 \%$ NW sideplates

cell 2004 d2o $-201 \%$ large area of d2o. used for filling lat.

lat 1504002 
1 0.0 .1

634. 0. 222222

cell 1810 fill $150-201202-203 \%$ inside pressure vessel $\%$

$\%$------ Void Outside Core -----

cell 90010 outside $201203 \%$ rest of universe cell 90020 outside $201-202 \%$ rest of universe cell 90030 outside $-201203 \%$ rest of universe cell 90040 outside $-201-202 \%$ rest of universe cell 90050 outside $201-203202 \%$ rest of universe

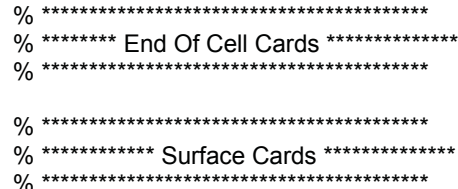

$\%{ }^{* * *}$ Fuel Plate Cylindrical surfaces Large Central Location **** surf 1 cyl $0.0 \quad 0.0 \quad 9.6000 \quad \%$ Fuel Cld inner Plate 1 surf 2 cyl $0.0 \quad 0.0 \quad 9.6375 \quad \%$ Fuel Mt inner Plate 1 $\begin{array}{lllll}\text { surf } 3 \mathrm{cyl} & 0.0 & 0.0 & 9.6875 \quad \% \text { Fuel Mt outer Plate } 1\end{array}$ surf $4 \mathrm{cyl} \quad 0.0 \quad 0.0 \quad 9.7250 \quad \%$ Fuel Cld outer Plate 1 surf 5 cyl $0.0 \quad 0.09 .9250 \quad \%$ Fuel Cld inner Plate 2 surf 6 cyl $0.0 \quad 0.0 \quad 9.9625 \quad \%$ Fuel Mt inner Plate 2 surf $7 \mathrm{cyl} \quad 0.0 \quad 0.0 \quad 10.0125 \quad \%$ Fuel Mt outer Plate 2

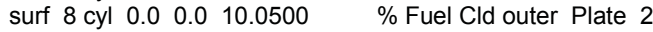
surf 9 cyl $0.0 \quad 0.0 \quad 10.2500 \quad \%$ Fuel Cld inner Plate 3 surf 10 cyl $\begin{array}{lll}0.0 & 0.0 & 10.2875 \quad \% \text { Fuel Mt inner Plate } 3\end{array}$ $\begin{array}{llll}\text { surf } 11 \text { cyl } & 0.0 & 0.0 & 10.3375\end{array} \%$ Fuel Mt outer Plate 3 surf 12 cyl $0.0 \quad 0.010 .3750 \quad \%$ Fuel Cld outer Plate 3 surf 13 cyl $0.0 \quad 0.010 .5750 \quad \%$ Fuel Cld inner Plate 4 surf 14 cyl $0.0 \quad 0.0 \quad 10.6125 \quad \%$ Fuel Mt inner Plate 4 surf 15 cyl $0.0 \quad 0.0 \quad 10.6625 \quad \%$ Fuel Mt outer Plate 4 surf 16 cyl $0.0 \quad 0.010 .7000 \quad \%$ Fuel Cld outer Plate 4 surf $\begin{array}{llll}17 \text { cyl } & 0.0 & 0.0 & 10.9000\end{array} \%$ Fuel Cld inner Plate 5 surf 18 cyl $\begin{array}{llll}0.0 & 0.0 & 10.9375 & \% \text { Fuel Mt inner Plate } 5\end{array}$ surf $19 \mathrm{cyl} \quad 0.0 \quad 0.0 \quad 10.9875 \quad \%$ Fuel Mt outer Plate 5 surf 20 cyl $0.0 \quad 0.011 .0250 \quad \%$ Fuel Cld outer Plate 5 surf 21 cyl $0.0 \quad 0.0 \quad 11.2250 \quad \%$ Fuel Cld inner Plate 6

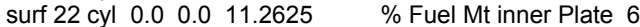
surf 23 cyl $0.0 \quad 0.0 \quad 11.3125 \quad \%$ Fuel Mt outer Plate 6 surf 24 cyl $0.0 \quad 0.011 .3500 \quad \%$ Fuel Cld outer Plate 6 surf 25 cyl $0.0 \quad 0.011 .5500 \quad \%$ Fuel Cld inner Plate 7 surf 26 cyl $0.0 \quad 0.0 \quad 11.5875 \quad \%$ Fuel Mt inner Plate 7 surf $27 \mathrm{cyl} \quad \begin{array}{llll}0.0 & 0.0 & 11.6375 \quad \% \text { Fuel Mt outer Plate } 7\end{array}$ surf 28 cyl $0.0 \quad 0.011 .6750 \quad \%$ Fuel Cld outer Plate 7 surf 29 cyl $0.0 \quad 0.011 .8750 \quad \%$ Fuel Cld inner Plate 8

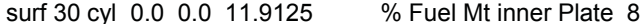
surf 31 cyl $0.0 \quad 0.0 \quad 11.9625 \quad \%$ Fuel Mt outer Plate 8 surf 32 cyl $\begin{array}{llll}0.0 & 0.0 & 12.0000 & \% \text { Fuel Cld outer Plate } 8\end{array}$ surf 33 cyl $0.0 \quad 0.0 \quad 12.2000 \quad \%$ Fuel Cld inner Plate 9 surf 34 cyl $\begin{array}{llll}0.0 & 0.0 & 12.2375 & \% \text { Fuel Mt inner Plate } 9\end{array}$ $\begin{array}{llll}\text { surf } 35 \text { cyl } & 0.0 & 0.0 & 12.2875\end{array} \%$ Fuel Mt outer Plate 9 surf 36 cyl $0.0 \quad 0.0 \quad 12.3250 \quad \%$ Fuel Cld outer Plate 9 surf 37 cyl $0.0 \quad 0.012 .5250 \quad \%$ Fuel Cld inner Plate 10 $\begin{array}{llll}\text { surf } 38 \text { cyl } & 0.0 & 0.0 & 12.5625\end{array} \%$ Fuel Mt inner Plate 10 surf 39 cyl $0.0 \quad 0.012 .6125 \quad \%$ Fuel Mt outer Plate 10 surf 40 cyl $0.0 \quad 0.012 .6500 \quad \%$ Fuel Cld outer Plate 10 surf 41 cyl $0.0 \quad 0.012 .8500 \quad \%$ Fuel Cld inner Plate 11 $\begin{array}{llll}\text { surf } 42 \text { cyl } & 0.0 & 0.0 & 12.8875\end{array} \%$ Fuel Mt inner Plate 11 surf 43 cyl $\begin{array}{llll}0.0 & 0.0 & 12.9375 & \% \text { Fuel Mt outer Plate } 11\end{array}$ surf 44 cyl $0.0 \quad 0.012 .9750 \quad \%$ Fuel Cld outer Plate 11 surf 45 cyl $0.0 \quad 0.0 \quad 13.1750 \quad \%$ Fuel Cld inner Plate 12 surf 46 cyl $\begin{array}{llll}0.0 & 0.0 & 13.2125 & \% \text { Fuel Mt inner Plate } 12\end{array}$ surf $47 \mathrm{cyl} \quad 0.0 \quad 0.0 \quad 13.2625 \quad \%$ Fuel Mt outer Plate 12 surf $\begin{array}{lllll}48 & \text { cyl } & 0.0 & 0.0 & 13.3000\end{array} \%$ Fuel Cld outer Plate 12

$\%$ *** vertical planes separating arcuate assemblies

surf 300 px 0.0

surf $301 \mathrm{px}-0.475$

surf 302 px 0.475

surf 303 py 0.0

surf 304 py -0.475

surf 305 py 0.475

surf 306 plane $-1.01 .00 .0 \quad 0.0$ 
surf 307 plane $-1.01 .00 .0-0.671751442$

surf 308 plane $-1.01 .00 .0 \quad 0.671751442$

surf 309 plane 1.01 .00 .00 .0

surf 310 plane $1.01 .00 .0-0.671751442$

surf 311 plane $1.01 .00 .0 \quad 0.671751442$

$\%$ *** Top and Bottom End Plates ***

surf $80 \mathrm{pz} 60$.

surf $81 \mathrm{pz}-60$. \% Bottom of fuel meat

surf $62 \mathrm{pz} 62$. \% Top of plates

surf $63 \mathrm{pz}-62$. \% Bottom of plates

surf 64 pz 82. \% Top of Top End Box

surf $65 \mathrm{pz}-82$. \% Bottom of Bottom End Box

$\%$ *** Large Test Space Surfaces ***

surf $121 \mathrm{cyl} \quad 0.0 \quad 0.06 .35 \%$ Outer surface of flux monitor holder

surf 122 cyl $0.0 \quad 0.0 \quad 6.95 \%$ Inner surface of pressure tube

surf 123 cyl $0.0 \quad 0.0 \quad 7.85 \%$ Outer surface of pressure tube

surf 124 cyl $0.0 \quad 0.07 .95 \%$ Inner surface of insulation jacket

surf 125 cyl $0.0 \quad 0.0 \quad 8.10 \%$ Outer surface of insulation jacket

surf 126 cyl $0.0 \quad 0.0 \quad 8.70 \%$ Inner surface of Al baffle

surf 127 cyl $0.0 \quad 0.0 \quad 9.40 \%$ Outer surface of Al baffle

surf 128 cyl $0.0 \quad 0.0 \quad 13.5 \%$ Inner surface of Pressure boundary Tube

surf 129 cyl $0.0 \quad 0.0 \quad 14.6 \%$ Outer surface of Pressure boundary Tube

$\% * * *$ Small Test Space Surfaces peripheral location ***

surf 220 cyl $0.0 \quad 0.0 \quad 3.54 \%$ Outer boundary of test space

surf 221 cyl $0.0 \quad 0.0 \quad 3.70 \%$ Outer surface of flow tube

surf 222 cyl $0.0 \quad 0.0 \quad 4.00 \%$ Inner surface of pressure tube

surf 223 cyl $0.0 \quad 0.0 \quad 4.90 \%$ Outer surface of pressure tube

surf 224 cyl $0.0 \quad 0.0 \quad 5.00 \%$ Inner surface of insulation jacket

surf 225 cyl $0.0 \quad 0.0 \quad 5.30 \%$ Outer surface of insulation jacket

surf 226 cyl $0.0 \quad 0.0 \quad 5.80 \%$ Inner surface of safety rod guide tube

surf 227 cyl $0.0 \quad 0.0 \quad 6.40 \%$ Outer surface of safety rod guide tube

surf 228 cyl $0.0 \quad 0.07 .50 \%$ Inner surface of safety rod follower

surf 229 cyl $0.0 \quad 0.0 \quad 8.30 \%$ Outer surface of safety rod follower

$\%{ }^{* * *} \mathrm{D} 2 \mathrm{O}$ tank outer boundary ***

surf 201 cyl $0.00 .0150 .0 \%$

surf $202 \mathrm{pz}-150$.

surf 203 pz 150 .

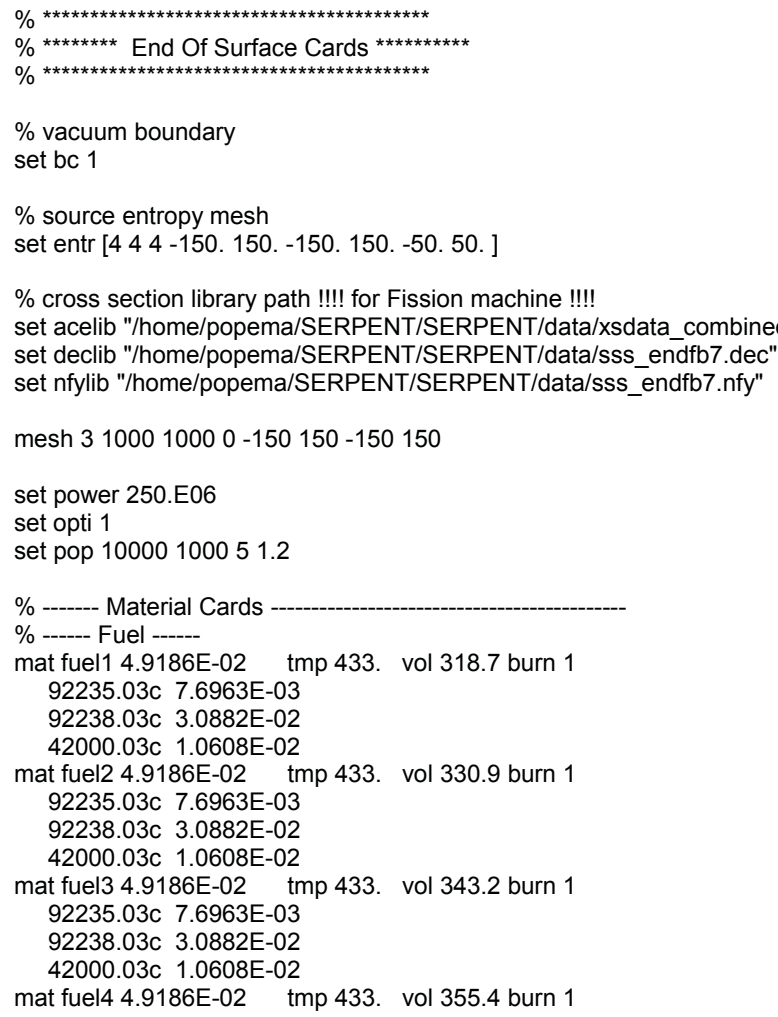


92235.03c 7.6963E-03 92238.03c 3.0882E-02 42000.03c 1.0608E-02

mat fuel5 4.9186E-02 tmp 433. vol 367.7 burn 1 92235.03c 7.6963E-03

$92238.03 \mathrm{C} 3.0882 \mathrm{E}-02$

42000.03c 1.0608E-02

mat fuel6 4.9186E-02 tmp 433. vol 379.9 burn 1 92235.03c 7.6963E-03

92238.03c 3.0882E-02

42000.03c 1.0608E-02

mat fuel7 4.9186E-02 tmp 433. vol 392.2 burn 1

92235.03c 7.6963E-03

92238.03c 3.0882E-02

42000.03 c 1.0608E-02

mat fuel8 4.9186E-02 tmp 433. vol 404.4 burn 1

92235.03c 7.6963E-03

92238.03c 3.0882E-02

42000.03 c 1.0608E-02

mat fuel9 4.9186E-02 tmp 433. vol 416.7 burn 1

92235.03c 7.6963E-03

92238.03 C 3.0882E-02

42000.03c 1.0608E-02

mat fuel10 4.9186E-02 tmp 433. vol 428.9 burn 1

92235.03c 7.6963E-03

92238.03 C 3.0882E-02

$42000.03 \mathrm{C} 1.0608 \mathrm{E}-02$

mat fuel11 4.9186E-02 tmp 433. vol 441.2 burn 1 92235.03c 7.6963E-03

92238.03c 3.0882E-02 42000.03c 1.0608E-02

mat fuel12 4.9186E-02 tmp 433. vol 453.4 burn 1 92235.03c 7.6963E-03

$92238.03 \mathrm{C} 3.0882 \mathrm{E}-02$

42000.03c 1.0608E-02

mat fuel13 4.9186E-02 tmp 433. vol 1951.8 burn 1

92235.03c 7.6963E-03

$92238.03 \mathrm{c} 3.0882 \mathrm{E}-02$

42000.03c 1.0608E-02

mat fuel14 4.9186E-02 tmp 433. vol 2026.9 burn 1

92235.03c 7.6963E-03

92238.03c 3.0882E-02

42000.03c 1.0608E-02

mat fuel15 4.9186E-02 tmp 433. vol 2101.9 burn 1 92235.03c 7.6963E-03

92238.03c 3.0882E-02

$42000.03 \mathrm{C}$ 1.0608E-02

mat fuel16 4.9186E-02 tmp 433. vol 2177.0 burn 1

92235.03c 7.6963E-03

$92238.03 \mathrm{C} 3.0882 \mathrm{E}-02$

$42000.03 \mathrm{C} 1.0608 \mathrm{E}-02$

mat fuel17 4.9186E-02 tmp 433. vol 2252.0 burn 1 92235.03 c 7.6963E-03

$92238.03 \mathrm{C} 3.0882 \mathrm{E}-02$

42000.03c 1.0608E-02

mat fuel18 4.9186E-02 tmp 433. vol 2327.1 burn 1 92235.03c 7.6963E-03

$92238.03 \mathrm{c} 3.0882 \mathrm{E}-02$

42000.03c 1.0608E-02

mat fuel19 4.9186E-02 tmp 433. vol 2402.1 burn 1

92235.03c 7.6963E-03

$92238.03 \mathrm{C} 3.0882 \mathrm{E}-02$

42000.03 c 1.0608E-02

mat fuel20 4.9186E-02 tmp 433. vol 2477.2 burn 1 92235.03c 7.6963E-03

92238.03 C 3.0882E-02

42000.03c 1.0608E-02

mat fuel21 4.9186E-02 tmp 433. vol 2552.2 burn 1 92235.03c 7.6963E-03 92238.03 C 3.0882E-02 $42000.03 \mathrm{c} 1.0608 \mathrm{E}-02$

mat fuel22 4.9186E-02 tmp 433. vol 2627.2 burn 1 92235.03c 7.6963E-03 92238.03 C 3.0882E-02 42000.03c 1.0608E-02

mat fuel23 4.9186E-02 tmp 433. vol 2702.3 burn 1 $92235.03 \mathrm{c}$ 7.6963E-03

$92238.03 \mathrm{C} 3.0882 \mathrm{E}-02$

$42000.03 \mathrm{C}$ 1.0608E-02

mat fuel24 4.9186E-02 tmp 433. vol 2777.3 burn 1 


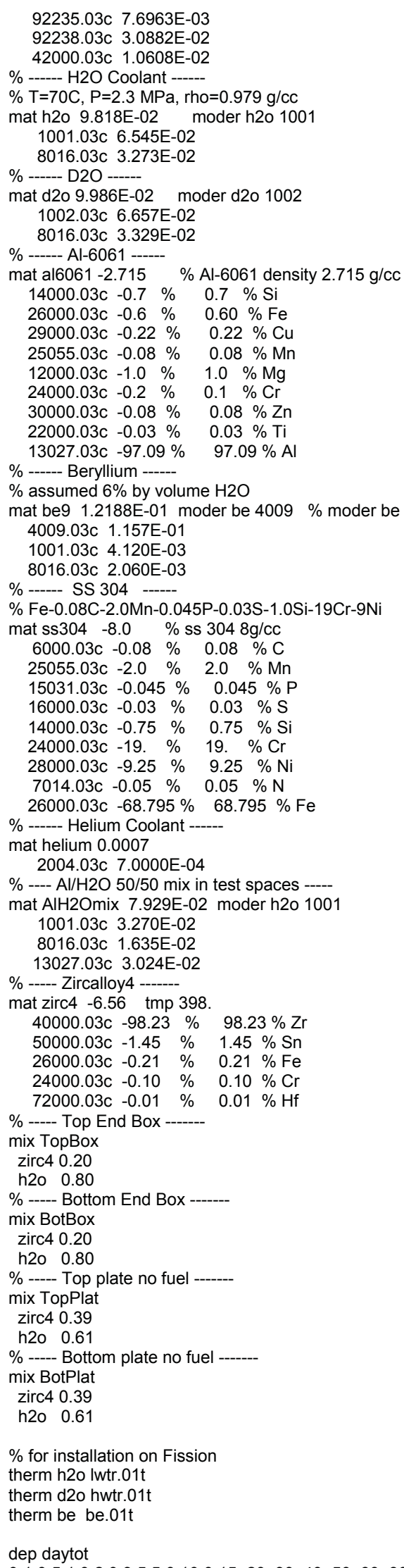

$\%$ assumed $6 \%$ by volume $\mathrm{H} 2 \mathrm{O}$

mat be9 1.2188E-01 moder be $4009 \%$ moder be 
$\%$ TALLIES

$\%$ Flux $1 \mathrm{MeV}$ cutoff in test zones

det CTrap dc $140 \mathrm{du} 1 \mathrm{dv} 432$. de $1 \mathrm{MeV} d x-3$. 3. 1 dy $-3.3 .1 \mathrm{dz}-60.60 .10$ det PTrap dc 120 du 2 dv 432. de $1 \mathrm{MeV}$ dx 31. 37. 1 dy -3. 3. $1 \mathrm{dz}-60.60 .10$

$\%$ Flux broken down into 3 .

det FTref1 dc 181 du 0 dv 192. de $1 \mathrm{MeV}$ dx 55.459 .41 dy $21.725 .71 \mathrm{dz}-60.60 .10$ det FTref2 dc 181 du 0 dv 192. de $1 \mathrm{MeV}$ dx 73.877 .81 dy $29.433 .41 \mathrm{dz}-60.60 .10$ det FTref3 dc 181 du 0 dv 192. de $1 \mathrm{MeV}$ dx 92.396 .31 dy $37.041 .01 \mathrm{dz}-60.60 .10$ det FTref4 dc 181 du 0 dv 192. de $1 \mathrm{MeV}$ dx $110.8114 .81 \mathrm{dy} 44.748 .71 \mathrm{dz}-60.60 .10$

ene $1 \mathrm{MeV}$ 11.0E-15 0.625E-06 1.020. 
Appendix B

ATR Core Diagrams 


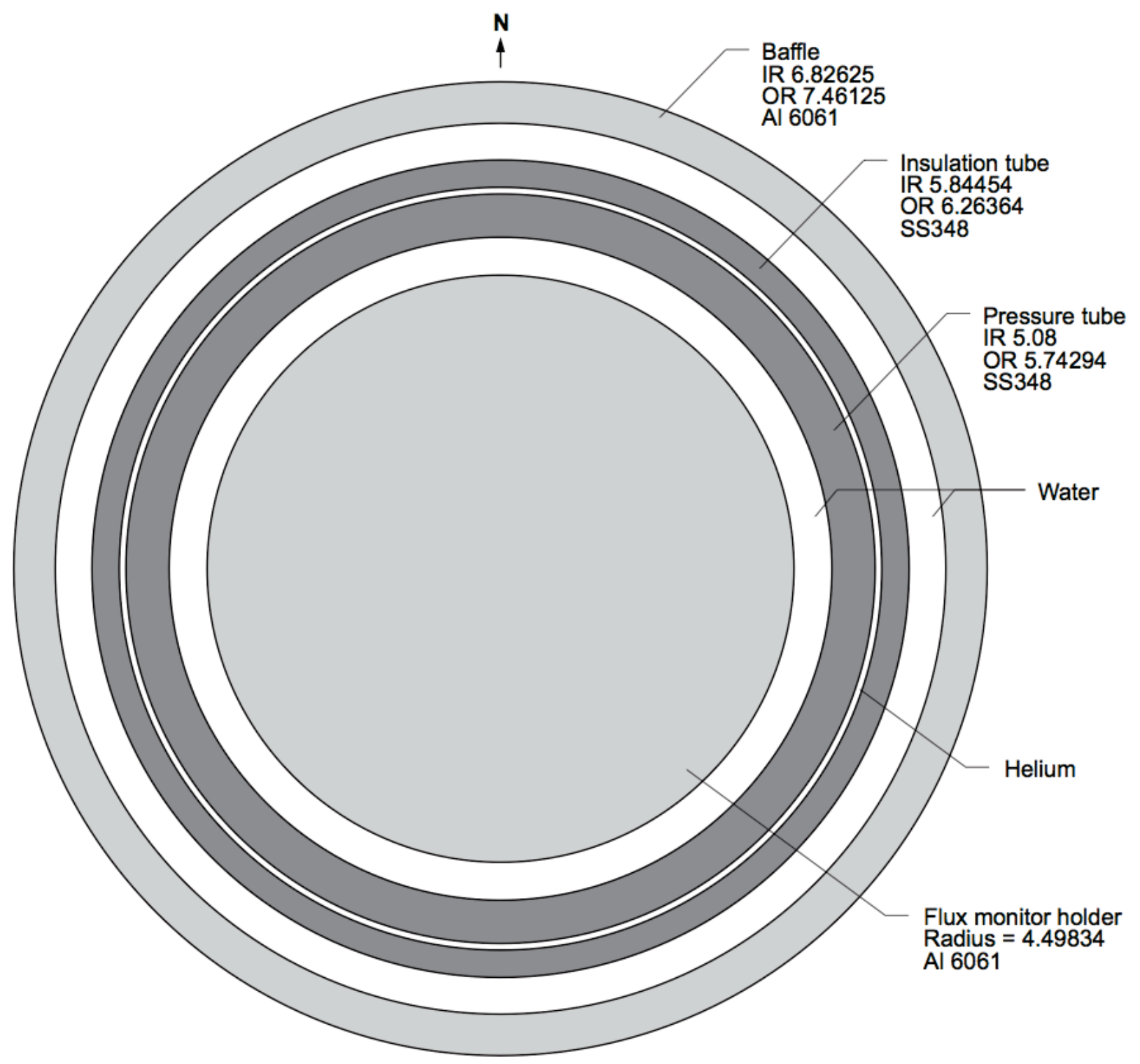

Dimensions in $\mathrm{cm}$

Figure C-1. Diagram of Northwest flux trap in ATR. 


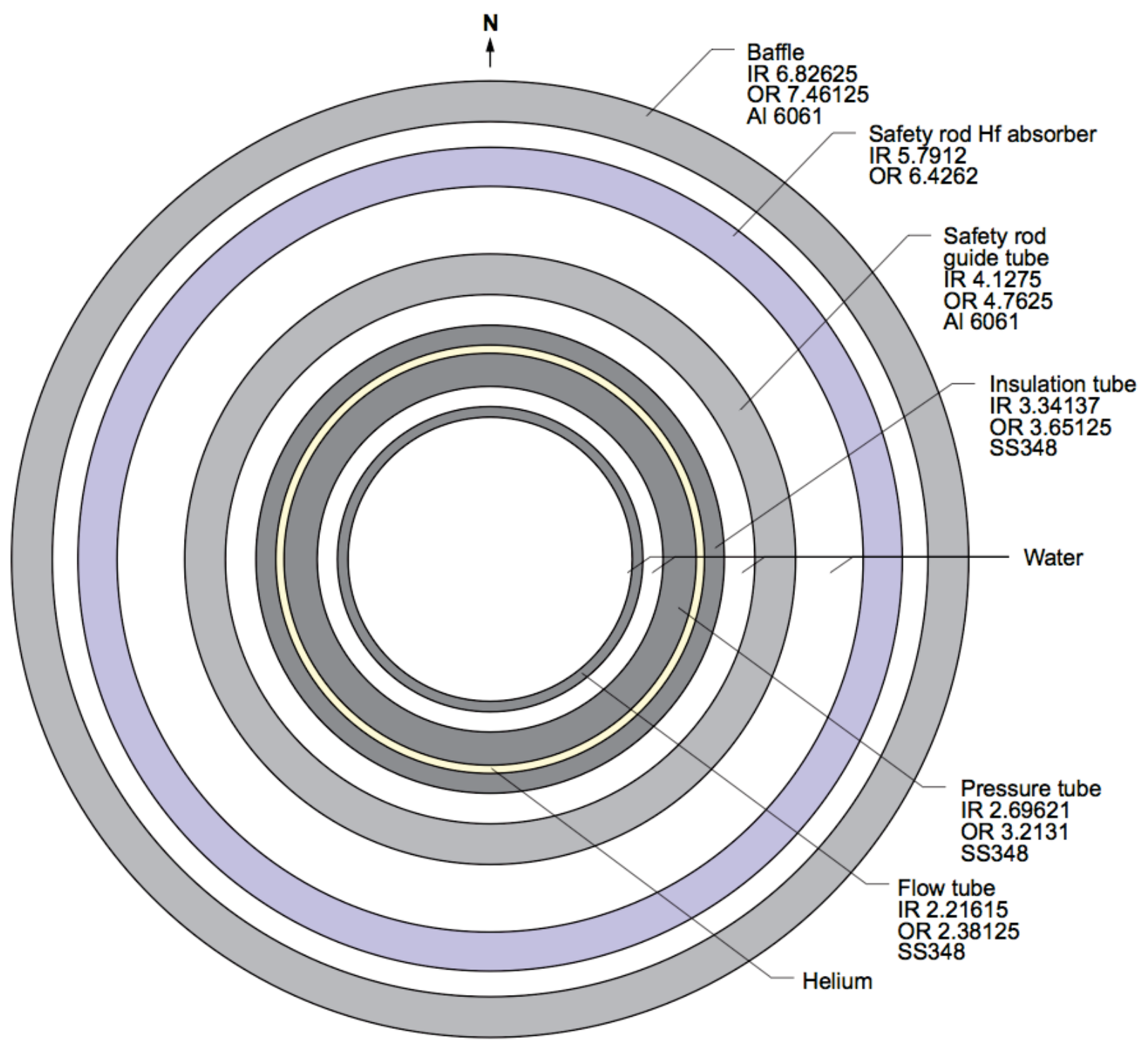

Dimensions in $\mathrm{cm}$

Figure C-2. Diagram of North, West, Southwest, and Southeast flux traps in ATR. 


\section{Appendix C Renderings of Cylindrical MATRIX Concept}




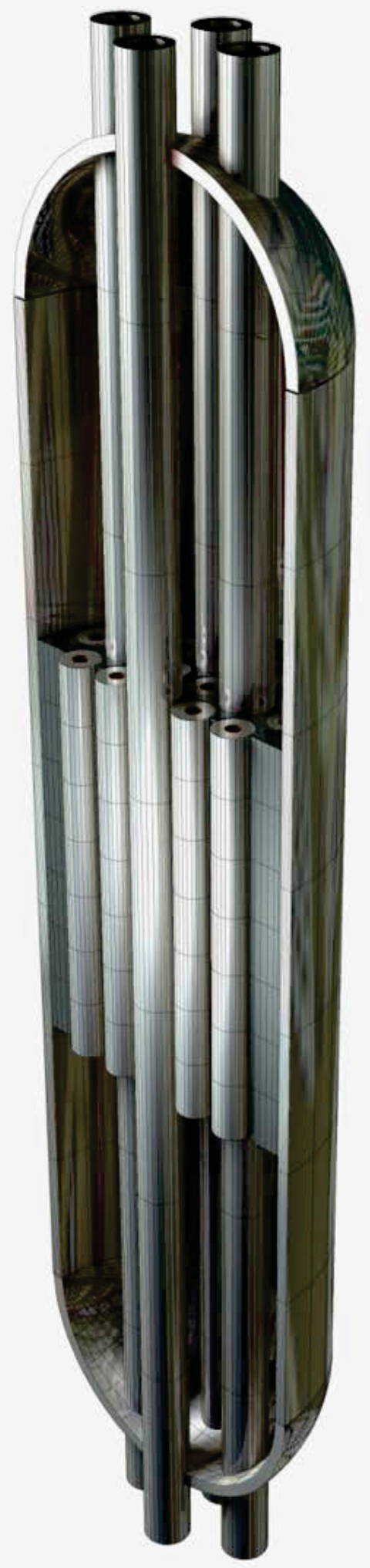

Figure C-1. Reactor vessel cut to show core internals. 


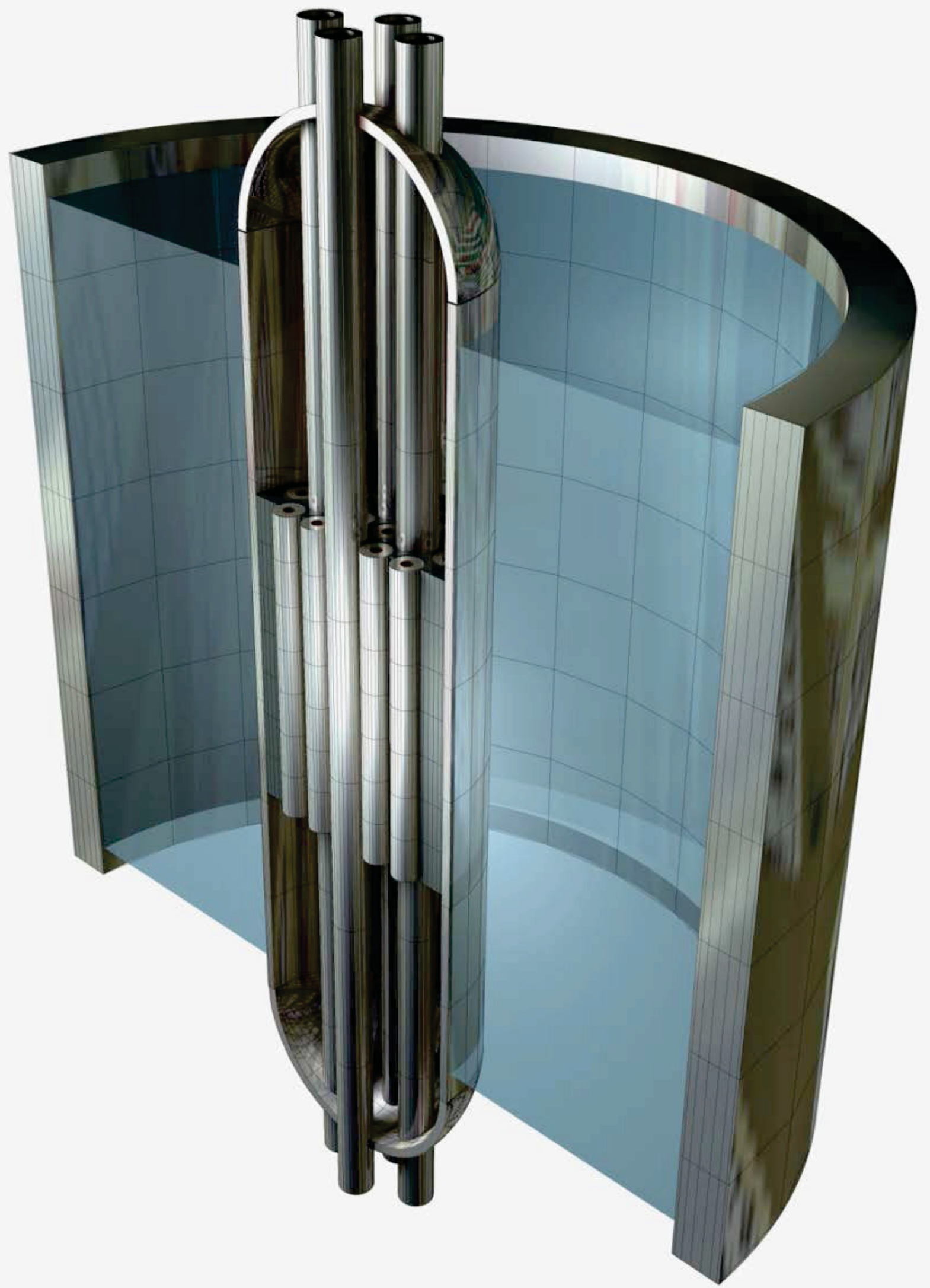

Figure $\mathrm{C}-2$. Vessel with surrounding $\mathrm{D}_{2} \mathrm{O}$ tank. 


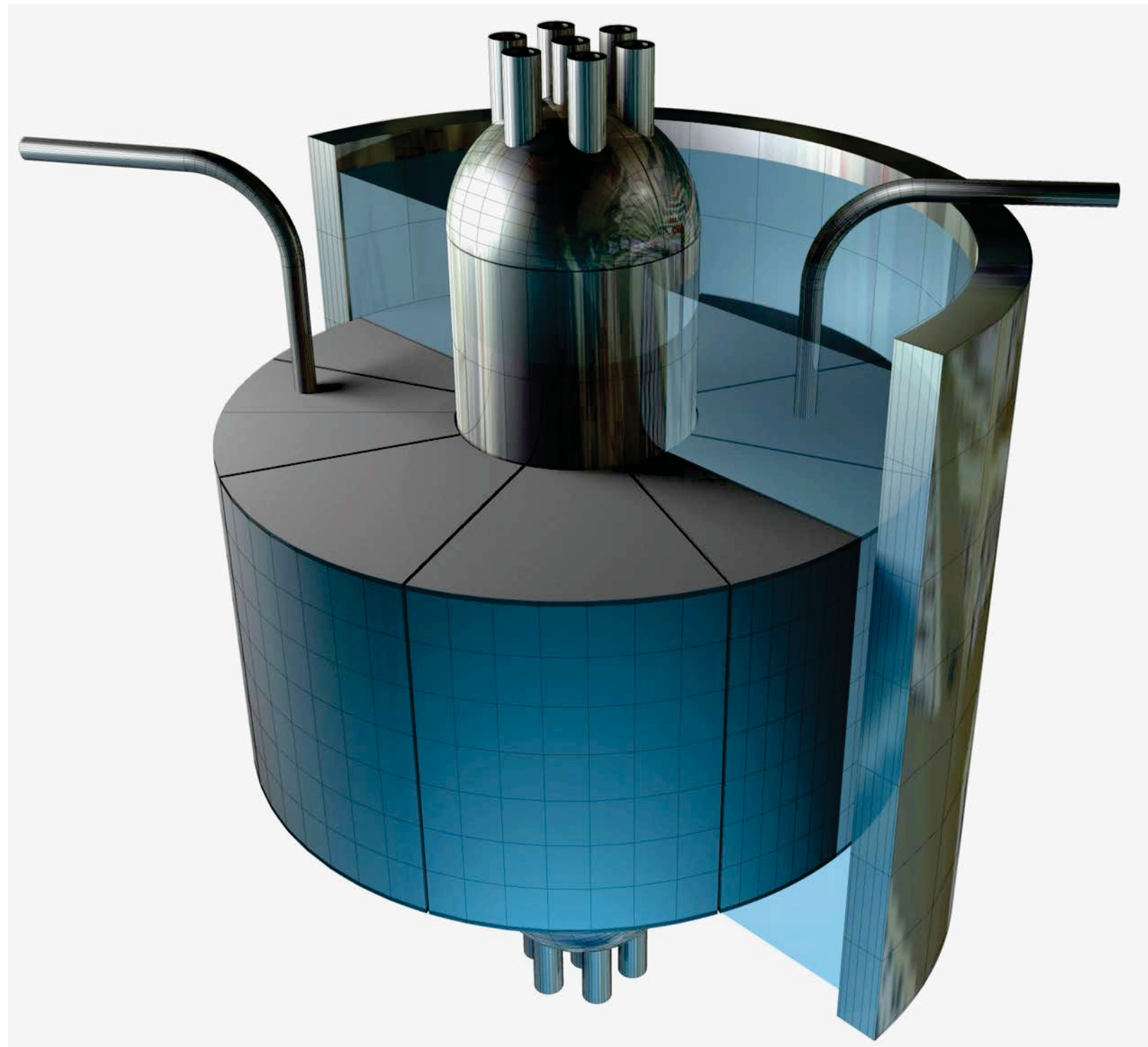

Figure C-3. Vessel with $\mathrm{D}_{2} \mathrm{O}$ tanks in water pool. 


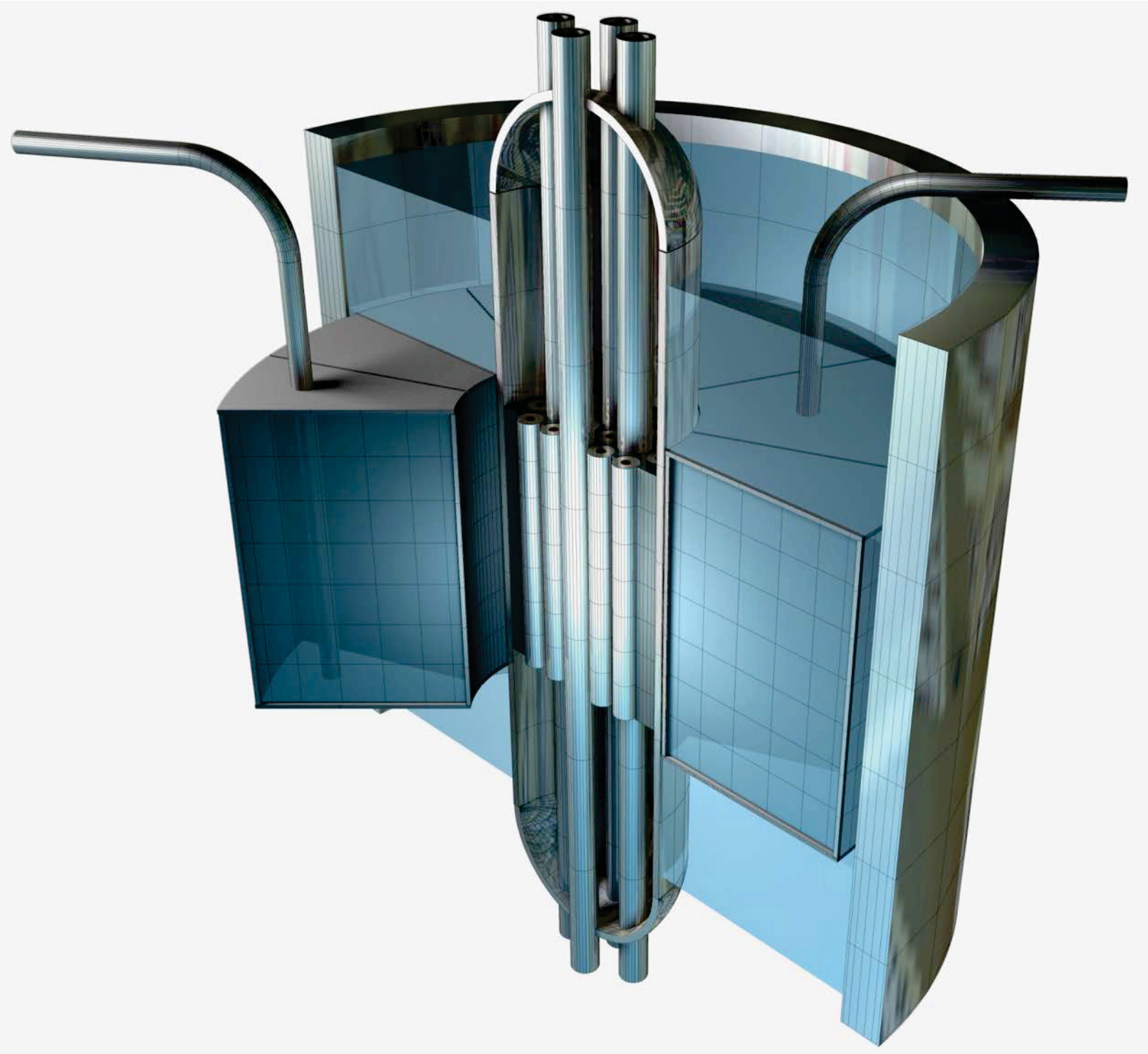

Figure C-4 Vessel with $\mathrm{D}_{2} \mathrm{O}$ tanks in water pool, both vessel and $\mathrm{D}_{2} \mathrm{O}$ tanks cut. 


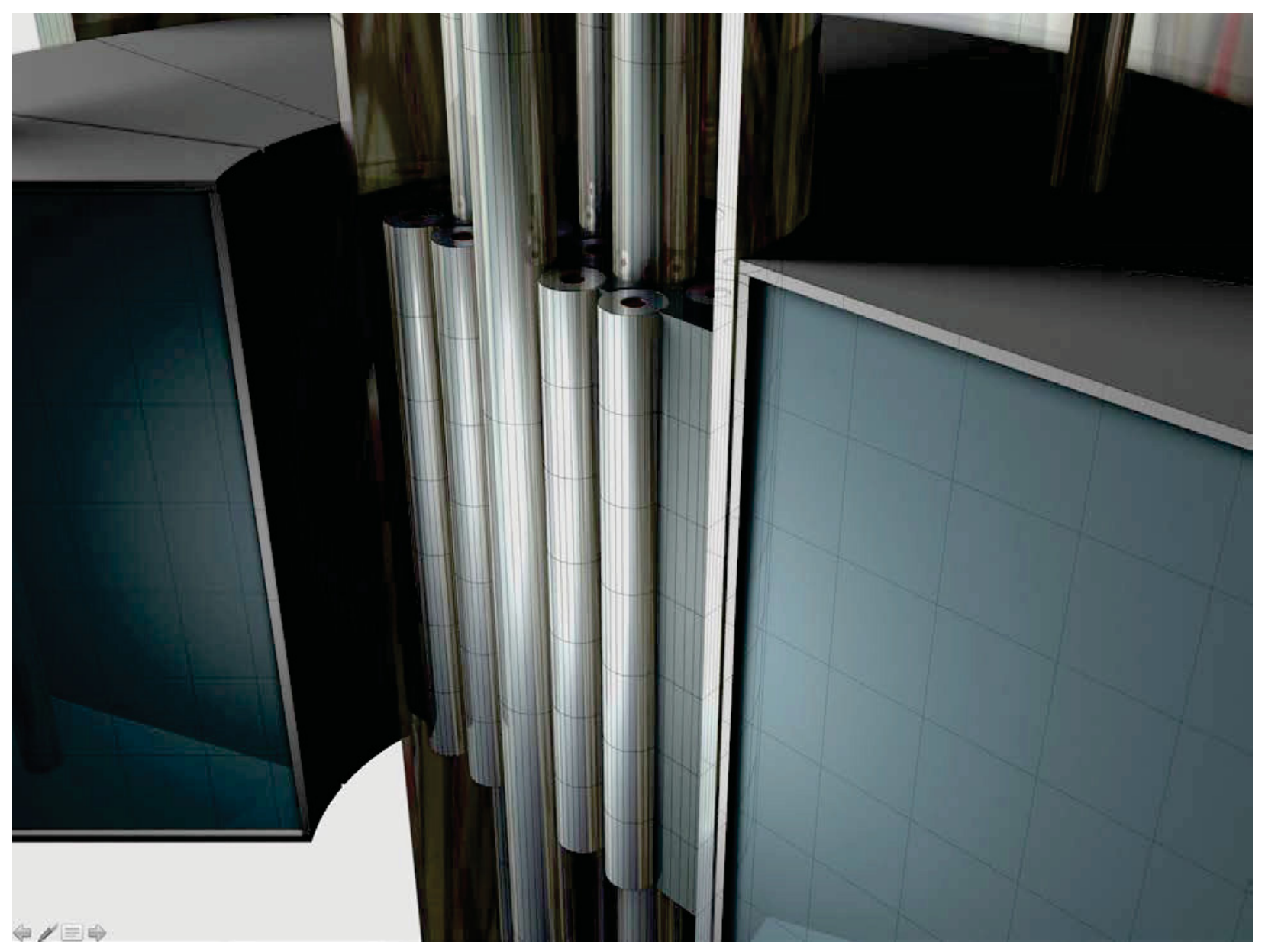

Figure C-5. Expanded view of core with vessel with $\mathrm{D}_{2} \mathrm{O}$ tanks in water pool. 